\title{
THE METHODS OF ALGEBRAIC TOPOLOGY FROM THE VIEWPOINT OF COBORDISM THEORY
}

\author{
S. P. NOVIKOV
}

\begin{abstract}
The goal of this work is the construction of the analogue to the Adams spectral sequence in cobordism theory, calculation of the ring of cohomology operations in this theory, and also a number of applications: to the problem of computing homotopy groups and the classical Adams spectral sequence, fixed points of transformations of period $p$, and others.
\end{abstract}

\section{INTRODUCTION}

In algebraic topology during the last few years the role of the so-called extraordinary homology and cohomology theories has started to become apparent; these theories satisfy all the Eilenberg-Steenrod axioms, except the axiom on the homology of a point. The merit of introducing such theories into topology and their first brilliant applications are due to Atiyah, Hirzebruch, Conner and Floyd, although in algebraic geometry the germs of such notions have appeared earlier (the Chow ring, the Grothendieck K-functor, etc.). Duality laws of Poincaré type, Thom isomorphisms, the construction of several important analogues of cohomology operations and characteristic classes, and also relations between different theories were quickly discovered and understood (cf. [2, 5, 8, 9, 11, 12]).

These ideas and notions gave rise to a series of brilliant results ([2]-[13]). In time there became manifest two important types of such theories: (1) theories of " $K$ type" and (2) theories of "cobordism type" and their dual homology ("bordism") theories.

The present work is connected mainly with the theory of unitary cobordism. It is a detailed account and further development of the author's work [19]. The structure of the homology of a point in the unitary cobordism theory was first discovered by Milnor [15] and the author [17]; the most complete and systematic account together with the structure of the ring can be found in [18]. Moreover, in recent work of Stong [22] and Hattori important relations of unitary cobordism to $K$-theory were found. We freely use the results and methods of all these works later, and we refer the reader to the works $[15,17,18,22]$ for preliminary information.

Our basic aim is the development of new methods which allow us to compute stable homotopy invariants in a regular fashion with the help of extraordinary homology theories, by analogy with the method of Cartan-Serre-Adams in the usual classical $Z_{p}$-cohomology theory. We have succeeded in the complete computation of the analogue of the Steenrod algebra and the construction of a "spectral sequence

Date: Received 10 APR 67.

Editor's note: Some small additions, contained in braces \{\} , have been made in translation. 
of Adams type" ${ }^{1}$ in some cohomology theories, of which the most important is the theory of $U$-cobordism, and we shall sketch some computations which permit us to obtain and comprehend from the same point of view a series of already known concrete results (Milnor, Kervaire, Adams, Conner-Floyd, and others), and some new results as well.

In the process of the work the author ran into a whole series of new and tempting algebraic and topological situations, analogues to which in the classical case are either completely lacking or strongly degenerate; many of them have not been considered in depth. All this leads us to express hope for the perspective of this circle of ideas and methods both for applications to known classical problems of homotopy theory, and for the formulation and solution of new problems from which one can expect the appearance of nontraditional algebraic connections and concepts.

The reader, naturally, is interested in the following question: to what extent is the program (of developing far-reaching algebraic-topological methods in extraordinary cohomology theory) able to resolve difficulties connected with the stable homotopy groups of spheres? In the author's opinion, it succeeds in showing some principal (and new) sides of this problem, which allow us to put forth arguments about the nearness of the problems to solution and the formulation of final answers. First of all, the question should be separated into two parts: (1) the correct selection of the theory of cobordism type as "leading" in this program, and why it is richer than cohomology and $K$-theory; (2) how to look at the problem of homotopy groups of spheres from the point of view of cobordism theory.

The answer to the first part of the question is not complicated. As is shown in Appendix 3, if we have any other "good" cohomology theory, then it has the form of cobordism with coefficients in an $\Omega$-module. Besides, working as in $\S \S 9$ and 12 , it is possible to convince oneself that these give the best filtrations for homotopy groups (at any rate, for complexes without torsion; for $p=2$ it may be that the appropriate substitute for $M U$ is $M S U$ ). In this way, the other theories lead to the scheme of cobordism theory, and there their properties may be exploited in our program by means of homological algebra, as shown in many parts of the present work.

We now attempt to answer the second fundamental part of the question. Here we must initially formulate some notions and assertions. Let $A_{p}^{U}\left[A^{U}\right]$ be the ring of cohomology operations in $U_{p}^{*}$-theory $\left[U^{*}\right.$, respectively], $\Lambda_{p}=U_{p}^{*}(P), \Lambda=$ $U^{*}(P), P=$ point, $Q_{p}=p$-adic integers. ${ }^{2}$ Note that $\Lambda \subset A^{U}$. The ring over $Q_{p}$, $\Lambda \otimes_{Z} Q_{p} \supset \Lambda_{p}$, lies in $A^{U} \otimes_{Z} Q_{p} \supset A_{p}^{U}$, and $\Lambda \otimes_{Z} Q_{p}$ is a local ring with maximal ideal $m \subset \Lambda \otimes_{Z} Q_{p}$, where $\Lambda \otimes_{Z} Q_{p} / m=Z_{p}$. Note that $\Lambda_{p}$ is an $A_{p}^{U}$-module and $A_{p}^{U}$ is also a left $\Lambda_{p}$-module.

\footnotetext{
${ }^{1}$ It may be shown that the Adams spectral sequence is the generalization specifically for $S$ categories (see $\S 1$ ) of "the universal coefficient formula," and this is used in the proofs of Theorems 1 and 2 of Appendix 3.

${ }^{2} U_{p}^{*}$-theory is a direct summand of the cohomology theory $U^{*} \otimes Q_{p}$, having spectrum $M_{p}$ such that $H^{*}\left(M_{p}, Z_{p}\right)=A /(\beta A+A \beta)$ where $A$ is the Steenrod algebra over $Z_{p}$ and $\beta$ is the Bokštern operator $\}$ (see $\S \S 1,5,11,12)$.
} 
Consider the following rings:

$$
\begin{gathered}
m_{p}=m \cap \Lambda_{p}, \quad \Lambda_{p} / m_{p}=Z_{p}, \\
\bar{\Lambda}_{p}=\sum_{i \geq 0} m_{p}^{i} / m_{p}^{i+1}, \\
\bar{A}=\bar{A}_{p}^{U}=\sum_{i \geq 0} m_{p}^{i} A_{p}^{U} / m_{p}^{i+1} A_{p}^{U},
\end{gathered}
$$

where $\bar{\Lambda}_{p}$ is an $\bar{A}$-module.

In this situation arises as usual a spectral sequence $\left(\tilde{\tilde{E}}_{r}, \tilde{\tilde{d}}_{r}\right)$, where

$$
\tilde{\tilde{E}}_{r} \searrow \operatorname{Ext}_{A^{U}}^{* *}(\Lambda, \Lambda) \otimes_{Z} Q_{p}, \quad \tilde{\tilde{E}}_{2}=\operatorname{Ext}_{\bar{A}_{p}^{U}}^{* * *}\left(\bar{\Lambda}_{p}, \bar{\Lambda}_{p}\right),
$$

determined by the maximal ideal $m_{p} \subset \Lambda_{p}$ and the induced filtrations.

It turns out that for all $p>2$ the following holds:

Theorem. The ring $\operatorname{Ext}_{\bar{A}_{p}^{U}}^{* * *}\left(\bar{\Lambda}_{p}, \bar{\Lambda}_{p}\right)$ is isomorphic to $\operatorname{Ext}_{A}^{* *}\left(Z_{p}, Z_{p}\right)$, and the algebraic spectral sequence $\left(\tilde{\tilde{E}}_{r}, \tilde{\tilde{d}}_{r}\right)$ is associated with the "geometric" spectral sequence of Adams in the theory $H^{*}\left(, Z_{p}\right)$. Here $p>2$ and $A$ is the usual Steenrod algebra for $Z_{p}$-cohomology.

We note that $\tilde{\tilde{E}}_{\infty}^{* * *}$ is associated with $\operatorname{Ext}_{A_{\tilde{U}}^{* *}}^{* *}(\Lambda, \Lambda) \otimes_{Z} Q_{p}$ (more precisely stated in $\S 12)$. A priori the spectral sequence $\left(\tilde{\tilde{E}}_{r}, \tilde{\tilde{d}}_{r}\right)$ is cruder than the Adams spectral sequence in $H^{*}\left(, Z_{p}\right)$-theory and $\tilde{\tilde{E}}_{\infty}^{* * *}$ is bigger than the stable homotopy groups of spheres; on account of this, the Adams spectral sequence for cobordism theory constructed in this work can in principle be non-trivial, since $\tilde{\tilde{E}}_{\infty}$ is associated with $\operatorname{Ext}_{A^{U}}(\Lambda, \Lambda) \otimes_{Z} Q_{p}$.

We now recall the striking difference between the Steenrod algebra modulo 2 and modulo $p>2$. As is shown in H. Cartan's well-known work, the Steenrod algebra for $p>2$ in addition to the usual grading possesses a second grading ("the number of occurrences of the Bokšteĭn homomorphism") of a type which cannot be defined for $p=2$ (it is only correct modulo 2 for $p=2$ ). Therefore for $p>2$ the cohomology $\operatorname{Ext}_{A}\left(Z_{p}, Z_{p}\right)$ has a triple grading in distinction to $p=2$. In $\S 12$ we show:

Lemma. There is a canonical algebra isomorphism

$$
\tilde{\tilde{E}}_{2}^{* * *}=\operatorname{Ext}_{\bar{A}_{p}^{U}}^{* *}\left(\bar{\Lambda}_{p}, \bar{\Lambda}_{p}\right)=\operatorname{Ext}_{A}^{* * *}\left(Z_{p}, Z_{p}\right) \quad \text { for } p>2 .
$$

From this it follows that the algebra $\tilde{\tilde{E}}_{2}$ for the "algebraic Adams spectral sequence" $\tilde{\tilde{E}}_{r}$ is not associated, but is canonically isomorphic to the algebra $\operatorname{Ext}_{A}\left(Z_{p}, Z_{p}\right)$ which is the second term of the usual topological Adams spectral sequence.

If we assume that existence of the grading of Cartan type is not an accidental result of the algebraic computation of the Steenrod algebra $A$, but has a deeper geometric significance, then it is not out of the question that the whole Adams spectral sequence is not bigraded, but trigraded, as is the term

$$
E_{2}=\operatorname{Ext}_{A}^{* * *}\left(Z_{p}, Z_{p}\right), \quad p \neq 2
$$


From this, obviously, would follow the corollary: for $p>2$ the algebraic Adams spectral sequence $\left(\tilde{\tilde{E}}_{r}, \tilde{\tilde{d}}_{r}\right)$ coincides with the topological Adams spectral sequence $\left(E_{r}, d_{r}\right)$, if the sequence $\left(E_{r}, d_{r}\right)$ is trigraded by means of the Cartan grading, as is $\left(\tilde{\tilde{E}}_{r}, \tilde{\tilde{d}}_{r}\right)$. Therefore the orders $\left|\pi_{N+i}\left(S^{N}\right)\right|$ would coincide with $\left|\sum_{t-s=i} \operatorname{Ext}_{A^{U}}^{s, t}(\Lambda, \Lambda)\right|$ up to a factor of the form $2^{h}$.

Moreover, this corollary would hold for all complexes without torsion (see $\S 12$ ).

The case $p=2$ is more complicated, although even there, there are clear algebraic rules for computing some differentials. This is indicated precisely in $\S 12$.

In this way it is possible not only to prove the nonexistence of elements of Hopf invariant one by the methods of extraordinary cohomology theory as in [4] (see also $\S \S 9,10)$, but also to calculate Adams differentials.

The content of this work are as follows: in $\S \S 1-3$ we construct the Adams spectral sequence in different cohomology theories and discuss its general properties.

$\S \S 4,5$ are devoted to cohomology operations in cobordism theory. Here we adjoin Appendices 1 and 2. This is the most important part of the work.

$\S \S 6,7$ are largely devoted to the computations of $U^{*}(M S U)$ and $\operatorname{Ext}_{A}^{* * U}\left(U^{*}(M S U), \Lambda\right)$.

$\S 8$ has an auxiliary character; in it we establish the facts from $K$-theory which we need.

$\S \S 10,11$ are devoted to computing $\operatorname{Ext}_{A^{U}}^{* *}(\Lambda, \Lambda)$.

$\S \S 9,12$ were discussed above; they have a "theoretical" character.

Appendices 3 and 4 are connected with the problems of fixed points and the problem of connections between different homology theories from the point of view of homological algebra. Here the author only sketches the proofs.

The paper has been constructed as a systematic exposition of the fundamental theoretical questions connected with new methods and their first applications. The author tried to set down and in the simplest cases to clarify the most important theoretical questions, not making long calculations with the aim of concrete applications; this is explained by the hope mentioned earlier for the role of a similar circle of ideas in further developments of topology.

\section{$\S 1$. The existence of the Adams spectral sequence in Categories}

Let $S$ be an arbitrary additive category in which $\operatorname{Hom}(X, Y)$ are abelian groups for $X, Y \in S$, having the following properties:

1. There is a preferred class of sequences, called "short exact sequences" $(0 \rightarrow$ $A \stackrel{g}{\rightarrow} B \stackrel{f}{\rightarrow} C \rightarrow 0)$, such that $f \cdot g=0$ and also:

a) the sequence $(0 \rightarrow 0 \rightarrow 0 \rightarrow 0 \rightarrow 0)$ is short exact;

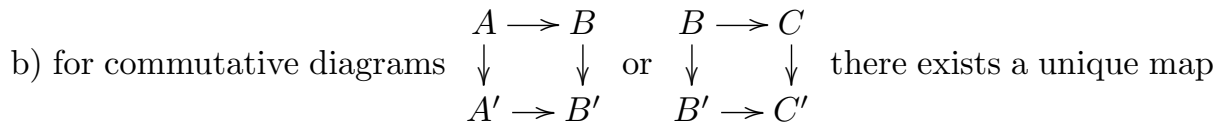
or short exact sequences

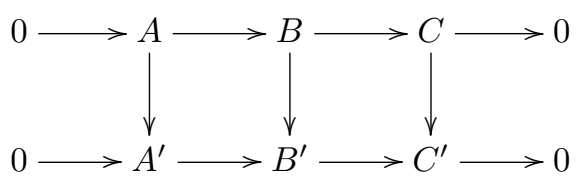

\{extending the given square\}; 
c) for any morphism $f: A \stackrel{f}{\rightarrow} B$ there exist unique short exact sequences $0 \rightarrow$ $C^{\prime} \rightarrow A \stackrel{f}{\rightarrow} B \rightarrow 0$ and $0 \rightarrow A \stackrel{f}{\rightarrow} B \rightarrow C \rightarrow 0$, where the objects $C$ and $C^{\prime}$ are related by a short exact sequence $0 \rightarrow C^{\prime} \rightarrow 0 \rightarrow C \rightarrow 0$ and $C$ and $C^{\prime}$ determine each other.

We introduce an operator $E$ in the category $S$ by setting $C^{\prime}=E^{-1} C$, or $C=$ $E C^{\prime}$, and we call $E$ the suspension.

Let $\operatorname{Hom}^{i}(X, Y)=\operatorname{Hom}\left(X, E^{i} Y\right)$ and $\operatorname{Hom}^{*}(X, Y)=\sum \operatorname{Hom}^{i}(X, Y)$.

2. For any short exact sequence $0 \rightarrow A \stackrel{f}{\rightarrow} B \stackrel{g}{\rightarrow} C \rightarrow 0$ and any $T \in S$ there are uniquely defined exact sequences

$$
\stackrel{\partial}{\rightarrow} \operatorname{Hom}^{i}(T, A) \stackrel{f_{*}}{\rightarrow} \operatorname{Hom}^{i}(T, B) \stackrel{g_{*}}{\longrightarrow} \operatorname{Hom}^{i}(T, C) \stackrel{\partial}{\rightarrow} \operatorname{Hom}^{i+1}(T, A)
$$

and

$$
\stackrel{\delta}{\rightarrow} \operatorname{Hom}^{i}(C, T) \stackrel{g^{*}}{\rightarrow} \operatorname{Hom}^{i}(B, T) \stackrel{f_{*}}{\rightarrow} \operatorname{Hom}^{i}(A, T) \stackrel{\delta}{\rightarrow} \operatorname{Hom}^{i+1}(C, T),
$$

which are functorial in $T$ and in $(0 \rightarrow A \rightarrow B \rightarrow C \rightarrow 0)$. Here the homomorphisms $f_{*}, g_{*}, f^{*}, g^{*}$ are the natural ones and the homomorphisms $\partial, \delta$ are induced by the projection $C \rightarrow E A$ in the short exact sequence $0 \rightarrow B \stackrel{g}{\rightarrow} C \rightarrow E A \rightarrow 0$ according to the above axiom 1.

3. In the category there exists a unique operation of direct sum with amalgamated subobjects: pairs $X, Y \in S$ and morphisms $Z \rightarrow X, Z \rightarrow Y$ define the sum $X+{ }_{Z} Y$ and the natural maps $X \rightarrow X+{ }_{Z} Y$ and $Y \rightarrow X+{ }_{Z} Y$ such that the following sequences are exact:

$$
\begin{gathered}
0 \rightarrow X \rightarrow X+{ }_{Z} Y \rightarrow C_{1} \rightarrow 0, \\
0 \rightarrow Y \rightarrow X+{ }_{Z} Y \rightarrow C_{2} \rightarrow 0
\end{gathered}
$$

(where $C_{1}$ and $C_{2}$ are defined by the exact sequences $0 \rightarrow Z \rightarrow X \rightarrow C_{2} \rightarrow 0$ and $0 \rightarrow Z \rightarrow Y \rightarrow C_{1} \rightarrow 0$ ). By definition we regard $X+{ }_{0} Y=X+Y$ where 0 is the point object\}.

Definition. We call two objects $X, Y \in S$ equivalent if there exists a third object $Z \in S$ and morphisms $f: X \rightarrow Z$ and $g: Y \rightarrow Z$ inducing isomorphisms of the functor $\operatorname{Hom}^{*}(Z$,$) with \operatorname{Hom}^{*}(X$,$) and \operatorname{Hom}^{*}(Y$,$) and of the functor \operatorname{Hom}^{*}(, Z)$ with $\operatorname{Hom}^{*}(, X)$ and $\operatorname{Hom}^{*}(, Y)$. We call the maps $f, g$ equivalences.

The transitivity of equivalences follows from the diagram

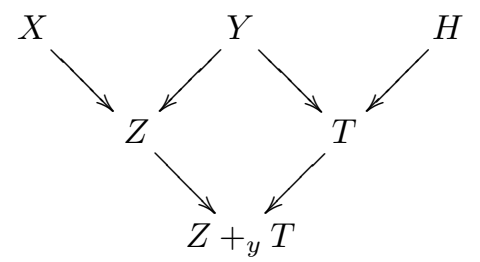

where all morphisms are equivalences (by virtue of the axiom on direct sums).

A spectrum in the category $S$ is given by a sequence $\left(X_{n}, f_{n}\right)$, where

$$
\begin{aligned}
f_{n}: E X_{n} \rightarrow X_{n+1} \quad \text { (direct spectrum), } \\
f_{n}: X_{n+1} \rightarrow E X_{n} \quad \text { (inverse spectrum). }
\end{aligned}
$$

By virtue of axioms 1 and 2 in the category $S$ there is a canonical isomorphism

$$
\operatorname{Hom}^{*}(X, Y)=\operatorname{Hom}^{*}(E X, E Y) \text {. }
$$


Therefore for spectra there are defined the compositions

$$
\begin{array}{cc}
f_{n+k-1} \ldots f_{n}: E^{k} X_{n} \rightarrow X_{n+k} & \text { (direct) } \\
f_{n} \ldots f_{n+k-1}: X_{n+k} \rightarrow E^{k} X_{n} & \text { (inverse) }
\end{array}
$$

which allow us to define passage to the cofinal parts of spectra.

For spectra $X=\left(X_{n}, f_{n}\right)$ and $Y=\left(Y_{n}, g_{n}\right)$ we define

$$
\operatorname{Hom}^{*}(X, Y)=\underset{n}{\lim _{n}} \underset{m}{\lim } \operatorname{Hom}^{*}\left(X_{n}, Y_{m}\right)
$$

in the case of direct spectra and

$$
\operatorname{Hom}^{*}(X, Y)=\underset{m}{\lim _{m}} \underset{n}{\lim } \operatorname{Hom}^{*}\left(X_{n}, Y_{m}\right)
$$

in the case of inverse spectra. Here, of course, let us keep in mind that in taking limits the grading in $\operatorname{Hom}^{*}($,$) is taken in the natural way. As usual, remember$ that the dimension of a morphism $E^{\gamma} T \rightarrow X_{n}$ is equal to $n+n_{0}-\gamma$, where $n_{0}$ is a fixed integer, given together with the spectrum, defining the dimension of the mappings into $X_{n}$, and usually considered equal to zero. In addition, Hom and Ext here and later are understood in the sense of the natural topology generated by spectra.

Thus arise categories $\vec{S}$ (direct spectra over $\mathrm{S}$ ) and $\overleftarrow{S}$ (inverse spectra). There are defined inclusions $S \rightarrow \vec{S}$ and $S \rightarrow \overleftarrow{S}$. We have the simple

Lemma 1.1. In the categories $\vec{S}$ and $\overleftarrow{S}$ there exist short exact sequences $0 \rightarrow A \rightarrow$ $B \rightarrow C \rightarrow 0$, where $A, B, C \in \vec{S}$ or $A, B, C \in \overleftarrow{S}$, satisfying axiom 1 of the category $S$ and axiom 2 for the functor $\operatorname{Hom}^{*}(T$,$) if A, B, C \in \vec{S}$ and $T \in \overleftarrow{S}$, and axiom 2 for $\operatorname{Hom}^{*}(, T)$ if $A, B, C \in \overleftarrow{S}$ and $T \in \vec{S}$. In the categories $\vec{S}$ and $\overleftarrow{S}$ there exist direct sums with amalgamation satisfying axiom 3 .

Proof. The existence of direct sums with amalgamation in the categories $\vec{S}$ and $\overleftarrow{S}$ is proved immediately.

Let us construct short exact sequences in $\vec{S}$. Let $A, B \in \vec{S}$ and $f: A \rightarrow B$ be a morphism in $\vec{S}$. By definition, $f$ is a spectrum of morphisms, hence is represented by a sequence $A_{n_{k}} \rightarrow B_{m_{k}}$ of maps. Consider the set of short exact sequences

$$
\left(0 \rightarrow C_{n_{k}} \rightarrow A_{n_{k}} \rightarrow B_{m_{k}} \rightarrow 0\right) \quad \text { and } \quad\left(0 \rightarrow A_{n_{k}} \rightarrow B_{m_{k}} \rightarrow C_{m_{k}}^{\prime} \rightarrow 0\right) .
$$

By axiom 1 of the category $S$ we have spectra in $\vec{S}, C=\left(C_{n_{k}}\right)$ and $C^{\prime}=\left(C_{m_{k}}^{\prime}\right)$ and morphisms $C \rightarrow A$ and $B \rightarrow C^{\prime}$. The corresponding sequences $0 \rightarrow C \rightarrow$ $A \rightarrow B \rightarrow 0$ and $0 \rightarrow A \rightarrow B \rightarrow C^{\prime} \rightarrow 0$ we call exact. Since passage to direct limit is exact, we have demonstrated the second statement of the lemma. For $\overleftarrow{S}$ analogously. Note that the spectra $C$ and $C^{\prime}$ are defined only up to equivalences of the following form: in $\vec{S}$ the equivalence is an isomorphism of functors $\operatorname{Hom}^{*}(T, C)$ and $\operatorname{Hom}^{*}\left(T, C^{\prime}\right)$; in $\overleftarrow{S}$ an isomorphism of $\operatorname{Hom}^{*}(C, T)$ and $\operatorname{Hom}^{*}\left(C^{\prime}, T\right)$

Obviously $C^{\prime}=E C$. This completes the proof of the lemma.

Definitions. a) Let $X \in \vec{S}$. The functor $\operatorname{Hom}^{*}(, X)$ is called a "cohomology theory" and is denoted by $X^{*}$.

b) Let $X \in \overleftarrow{S}$. The functor $\operatorname{Hom}^{*}(X$,$) is called a "homology theory" and is$ denoted by $X_{*}$. 
c) The ring $\operatorname{Hom}^{*}(X, X)$ for $X \in \vec{S}$ is called "the Steenrod ring" for the cohomology theory $X^{*}$. Analogously we obtain the Steenrod ring $\operatorname{Hom}^{*}(X, X)$ for $X \in \widehat{S}$ (homology theory).

d) The Steenrod ring for the cohomology theory $X^{*}$ is denoted by $A^{X}$, for the homology theory by $A_{X}$. They are graded topological rings with unity.

Note that an infinite direct $\operatorname{sum} Z=\sum X_{i}$ of objects $X_{i} \in \vec{S}$ lies, by definition,

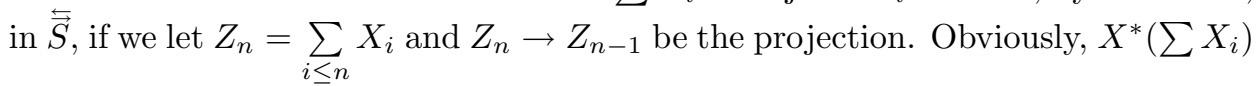
is an infinite-dimensional free $A^{X}$-module, being the limit of the direct spectrum

$$
\operatorname{Hom}^{*}\left(Z_{n}, X\right) \rightarrow \operatorname{Hom}^{*}\left(Z_{n+1}, X\right)
$$

where $X \in \vec{S} \subset \overleftarrow{\bar{S}}$, all $X_{i}$ are equivalent to the object $X$ or $E^{\gamma_{i}} X$, and $E$ is the suspension.

For an homology theory, if $X \in \vec{S}$, an infinite direct $\operatorname{sum} \sum X_{i}$ is considered as the limit of the direct spectrum

$$
\cdots \rightarrow \sum_{i \leq n} X_{i} \rightarrow \sum_{i \leq n+1} X_{i} \rightarrow \ldots,
$$

where $X_{i}$ is $E^{\gamma_{i}} X$, and therefore lies in $\overleftarrow{\bar{S}}$, and the $A_{X}$-module $\operatorname{Hom}^{*}\left(X, \sum X_{i}\right)$ is free.

By $X$-free objects for $X \in \vec{S}$ we mean direct sums $\sum X_{i}$, where $X_{i}=E^{\gamma_{i}} X$ for arbitrary integers $\gamma_{i}$. Finite direct sums belong to $\vec{S}$.

There are simple properties which give the possibility of constructing the Adams spectral sequence by means of axioms 1-3 for the category $S$.

For any object $T \in \overleftarrow{S}$ and any $X$-free object $Z \in \vec{S}$ we have

$$
\operatorname{Hom}^{*}(T, Z)=\operatorname{Hom}_{A^{X}}^{*}\left(X^{*}(Z), X^{*}(T)\right) .
$$

Let us give some definitions.

1) For an object $Y \in S$ we understand by a filtration in the category an arbitrary sequence of morphisms

$$
Y=Y_{-1} \stackrel{f_{0}}{\longleftarrow} Y_{0} \leftarrow Y_{1} \leftarrow \ldots \stackrel{f_{i}}{\longleftarrow} Y_{i} \leftarrow \ldots
$$

2) The filtration will be called $X$-free for $X \in \vec{S}$ if $Z_{i} \in \vec{S}$ are $X$-free objects such that there are short exact sequences

$$
0 \rightarrow Y_{i} \stackrel{f_{i}}{\longrightarrow} Y_{i-1} \stackrel{g_{i}}{\longrightarrow} Z_{i} \rightarrow 0, \quad Y_{-1}=Y .
$$

3) By the complexes associated with the filtration, for any $T \in \bar{S}$, are meant the complexes $\left(C_{x}, \partial_{x}\right)$ and $\left(B_{T}, \delta_{T}\right)$, where $\left(C_{x}\right)_{i}=X^{*}\left(Z_{i}\right)$ and $\left(B_{T}\right)^{i}=T_{*}\left(Z_{i}\right)$ and the differentials $\partial:\left(C_{x}\right)_{i} \rightarrow\left(C_{x}\right)_{i-1}$ and $\delta_{T}\left(B_{T}\right)_{i} \rightarrow\left(B_{T}\right)_{i+1}$ are the compositions

$$
\partial_{X}: X^{*}\left(Z_{i}\right) \stackrel{g_{i}}{\longrightarrow} X^{*}\left(Y_{i-1}\right) \stackrel{\delta}{\rightarrow} X^{*}\left(Z_{i-1}\right)
$$

and

$$
\delta_{T}: T_{*}\left(Z_{i}\right) \stackrel{\partial}{\rightarrow} T_{*}\left(Y_{i}\right) \stackrel{g_{i+1}{ }^{*}}{\longrightarrow} T_{*}\left(Z_{i+1}\right) .
$$

4) An $X$-free filtration is called acyclic if $\left(C_{x}, \partial_{x}\right)$ is acyclic in the sense that $H_{0}\left(C_{x}\right)=X^{*}(Y)$ and $H_{i}\left(C_{x}\right)=0$ for $i>0$.

From the properties (axioms 1 and 2) of the category $S$ and Lemma 1.1 we obtain the obvious 
Lemma 1.2. 1) Each filtration $\left(Y \leftarrow Y_{0} \leftarrow Y_{1} \leftarrow \ldots\right)$ defines a spectral sequence $\left(E_{r}, d_{r}\right)$ with term $E_{1}=B_{T}, d_{1}=\delta_{T}$, associated with $\operatorname{Hom}^{*}(T, Y)$ in the sense that there are defined homomorphisms $q_{0}: \operatorname{Hom}^{*}(T, Y) \rightarrow E_{\infty}^{0, *}, q_{i}: \operatorname{Ker} q_{i-1} \rightarrow E_{\infty}^{i, *}$, where the filtration $\left(\operatorname{Ker} q_{i}\right)$ in $T_{*}(Y)=\operatorname{Hom}^{*}(T, Y)$ is defined by the images of compositions of filtration maps $T_{*}\left(Y_{i}\right) \rightarrow T_{*}(Y)$.

2) If the filtration is $X$-free, the complex $\left(B_{T}, \delta_{T}\right)$ is precisely $\operatorname{Hom}_{A^{X}}^{*}\left(C_{x}, X^{*}(T)\right)$ $\left\{\right.$ with differential $\left.\operatorname{Hom}_{A^{X}}\left(\partial_{x}, 1\right)\right\}$.

3) If the filtration is $X$-free and acyclic, then $E_{2}^{* *}$ in this spectral sequence coincides precisely with $\operatorname{Ext}_{A^{T}}^{* *}\left(X^{*}(Y), X^{*}(T)\right)$.

Lemma 1.2 follows in the obvious way from axioms 1,2 of the category $S$ and Lemma 1.1.

However, the problem of the existence of $X$-free and acyclic filtrations is nontrivial. We shall give their construction in a special case, sufficient for our subsequent purposes.

Definition 1.1. The spectrum $X \in \vec{S}$ will be called stable if for any $T \in S$ and any $j$ there exists an integer $n$ such that $\operatorname{Hom}^{s}\left(T, X_{m}\right)=\operatorname{Hom}^{m}(T, X)$ for all $m \geq n$, $s \geq j$.

Definition 1.2. The cohomology theory $X^{*}, X \in \vec{S}$, defined by a stable spectrum $X$ will be called Noetherian if for all $T \in S$ the $A^{X}$-module $X^{*}(T)$ is finitely generated over $A^{X}$.

We have

Lemma 1.3. If $X^{*}$ is a Noetherian cohomology and $Y \in S$, then there exists a filtration

$$
Y \leftarrow Y_{0} \leftarrow \cdots \leftarrow Y_{i-1} \leftarrow Y_{i} \leftarrow \ldots
$$

such that $Z_{i}=Y_{i-1} / Y_{i}$ is a direct sum $Z_{i}=\sum_{j} X_{n_{j}}$ for large $n_{j}$ and the complex $C=\sum X^{*}\left(Z_{i}\right)$ is acyclic through large dimensions. Here $X=\left(X_{n}\right) \in \vec{S}$.

Proof. Take a large integer $n$ and consider a map $Y \rightarrow \sum_{i} X_{n}^{(i)}$ such that $X^{*}\left(\sum_{i} X_{n}\right) \rightarrow X^{*}(Y)$ is an epimorphism, where $X^{*}$ is a Noetherian cohomology theory.

By virtue of the stability of the spectrum $X$, for $Y \in S$ there is an integer $n$ such that the map $Y \rightarrow \sum X_{i}$ factors into the composition $Y \stackrel{f_{0}}{\longrightarrow} \sum_{i} X_{n} \rightarrow \sum E^{i} X$, where $X_{n} \rightarrow X$ is the natural map. Therefore $X^{*}\left(\sum X_{n_{i}}\right) \rightarrow X^{*}(Y) \stackrel{X^{*}\left(f_{0}\right)}{\longrightarrow} X^{*}(Y)$ is an epimorphism. Consider the short exact sequence

$$
0 \rightarrow Y_{0}^{(n)} \rightarrow Y \stackrel{f_{0}}{\longrightarrow} \sum_{i} X_{n} \rightarrow 0 .
$$

Obviously $X^{*}\left(Y_{0}^{(n)}\right)=\operatorname{Ker} X^{*}\left(f_{0}\right)$ and $Y_{0}^{(n)} \in S$. Now take a large number $n_{1} \gg n$ and do the same to $Y_{0}^{(n)}$ as was done to $Y$, and so on. We obtain a filtration

$$
Y \leftarrow Y_{0}^{(n)} \leftarrow Y_{1}^{\left(n, n_{1}\right)} \leftarrow Y_{2}^{\left(n, n_{1}, n_{2}\right)} \leftarrow \ldots,
$$

where the $Z_{i}$ are sums of objects of the form $\sum X_{m_{k}}$, with $m_{k}$ very large.

By definition, $C=\sum_{i} X^{*}\left(Z_{i}\right)$ is an acyclic complex through large dimensions. 
Definition 1.3. A stable spectrum $X=\left(X_{n}\right)$ in the category $\vec{S}$ is called acyclic if for each object $T \in S$ we have the equalities:

a) $\operatorname{Ext}_{A^{X}}^{i, t}\left(X^{*}\left(X_{n}\right), X^{*}(T)\right)=0, i>0, t-i<f_{n}(i)$, where $f_{n}(i) \rightarrow \infty$ as $n \rightarrow \infty$;

b) $\operatorname{Hom}_{A^{X}}^{t}\left(X^{*}\left(X_{n}\right), X^{*}(T)\right)=\operatorname{Hom}^{t}(T, X)$ for $t<f_{n}$, and $f_{n} \rightarrow \infty$ as $n \rightarrow \infty$.

The so-called Adams spectral sequence $\left(E_{r}, d_{r}\right)$ with $E_{2}$-term $E_{2}=$ $\operatorname{Ext}_{A^{X}}^{* *}\left(X^{*}(Y), X^{*}(T)\right)$ arises in the following cases:

1. If in the category $\vec{S}$ there exists an $X$-free acyclic filtration $Y=Y_{-1} \leftarrow Y_{0} \leftarrow$ $Y_{1} \leftarrow \cdots \leftarrow Y_{i-1} \leftarrow Y_{i} \ldots$, on the basis of Lemma 1.2. However, such a filtration does not always exist, since the theory $X^{*}$ in the category $\vec{S}$ does not have the exactness property.

2. If $Y \in S, T \in S$ and the theory $X^{*}$ is stable, Noetherian and acyclic, then, by virtue of Lemma 1.3, there exists a filtration

$$
Y_{-1}=Y \leftarrow Y_{0} \leftarrow Y_{1} \leftarrow \cdots \leftarrow Y_{i} \leftarrow \ldots,
$$

where the $Y_{i} / Y_{i+1}$ are sums of objects $X_{n}$, for numbers $n$ which may be taken as large as we want, with the filtration acyclic through large gradings. For such a filtration, the corresponding spectral sequence $\left(E_{r}, d_{r}\right)$ has the term $E_{2}^{s, t}=$ Ext $_{A^{X^{s, t}}}\left(X^{*}(Y), X^{*}(T)\right)$ through large gradings, by the definition of acyclicity for the theory $X^{*}$.

In this way we obtain:

Theorem 1.1. For any stable Noetherian acyclic cohomology theory $X \in \vec{S}$ and objects $Y, T \in S$, one can construct an Adams spectral sequence $\left(E_{r}, d_{r}\right)$, where $d_{r}: E_{r}^{s, t} \rightarrow E_{r}^{s+r, t+r-1}$ and the groups $\sum_{t-s=m} E_{\infty}^{s, t}$ are connected to $\operatorname{Hom}^{m}(t, Y)$ in the following way: there exist homomorphisms

$$
q_{i}: \text { Ker } q_{i-1} \rightarrow E_{\infty}^{i, i+m}, \quad i \geq 0,
$$

where

is the natural homomorphism.

$$
q_{0}: \operatorname{Hom}^{m}(T, Y) \rightarrow \operatorname{Hom}_{A^{X}}^{m}\left(X^{*}(Y), X^{*}(T)\right)
$$

The Adams spectral sequence is functorial in $T$ and $Y$.

Remark 1.1. The homomorphism $q_{1}$ : $\operatorname{Ker} q_{0} \rightarrow \operatorname{Ext}_{A^{X}}^{1, *}\left(X^{*}(Y), X^{*}(T)\right)$ is called the "Hopf invariant."

Remark 1.2. For objects $T, Y \in S$ and a stable Noetherian acyclic homology theory $X \in \bar{S}$ one can also construct an Adams spectral sequence $\left(E_{r}, d_{r}\right)$ such that $E_{2}=\operatorname{Ext}_{A_{X}}^{* *}\left(X_{*}(T), X_{*}(Y)\right)$. In this spectral sequence, $d_{r}: E_{r}^{p, q} \rightarrow E_{r}^{p-r, q+r+1}$, and the homomorphisms $q_{i}$ are such that

$$
q_{i}: \operatorname{Ker} q_{i-1} \rightarrow E_{\infty}^{i, i+n},
$$

where

$$
q_{0}: \operatorname{Hom}^{n}(T, Y) \rightarrow \operatorname{Hom}_{A^{X}}^{n}\left(X^{*}(Y), X^{*}(T)\right)
$$

is the natural homomorphism and $A_{X}$ is the Steenrod ring of the homology theory $X_{*}$.

The proof of Theorem 1.1 is a trivial consequence of Lemmas 1.1-1.3 and standard verifications of the functoriality of the spectral sequence in the case where the filtration is $X$-free and acyclic. 
We shall be specially interested in those cases when the Adams spectral sequence converges exactly to $T_{*}(Y)=\operatorname{Hom}^{*}(T, Y)$. Let us formulate a simple criterion for convergence:

(A) If there exists an $X$-free filtration $Y_{-1}=Y \leftarrow Y_{0} \leftarrow \cdots \leftarrow Y_{i}$ (not necessarily acyclic) such that for any $j, l$ there exists a number $i>l$, depending on $j$ and $l$, for which $\sum_{k<j} \operatorname{Hom}^{k}\left(T, Y_{i}\right)=0$, then the Adams spectral sequence converges exactly to $\operatorname{Hom}^{*}(T, Y)$. Criterion (A) does not appear to be the most powerful of those possible, but it will be fully sufficient for the purposes of the present work.

\section{$\S 2$. The $S$-Category of Finite COMplexes With Distinguished Base POINTS. Simplest OPERATIONS IN THIS CATEGORY}

The basic categories we shall be dealing with are the following:

1. The $S$-category of finite complexes and the categories $\vec{S}$ and $\overleftarrow{S}$ over it.

2. For any flat $Z$-module $G$ (an abelian group such that $\otimes_{Z} G$ is an exact functor) we introduce the category $S \otimes_{Z} G$, in which we keep the old objects of $S$ and let $\operatorname{Hom}(X, Y) \otimes_{Z} G$ be the group of morphisms of $X$ to $Y$ in the new category $S \otimes_{Z} G$. Important examples are: a) $G=Q$, b) $G=Q_{p}$ (p-adic integers). The respective categories will be denoted by $S_{0}$ for $G=Q$ and $S_{p}$ for $G=Q_{p}, p$ a prime.

3. In $S$ (or $S_{p}$ for $p>0$ ) we single out the subcategory $D$ (or $D_{p} \subset S_{p}$ ) consisting of complexes with torsion-free integral cohomology. It should be noted that the subcategories $D$ and $D_{p}$ are not closed with respect to the operations entering in axiom 1 for $S$-categories.

These subcategories, however, are closed with respect to the operations referred to, when the morphism $f: A \rightarrow B$ is such that $f^{*}: H^{*}(B, Z) \rightarrow H^{*}(A, Z)$ is an epimorphism.

Therefore the category $D$ is closed under the construction of $X$-free acyclic resolutions (only acyclic), and it is possible to study the Adams spectral sequence only for $X, Y \in D\left(\right.$ or $\left.D_{p}\right)$.

The following operations are well known in the $S$-category of spaces of the homotopy type of finite complexes (with distinguished base points):

1. The connected sum with amalgamated subcomplex $X+{ }_{Z} Y$, becoming the wedge $X \vee Y$ if $Z=0$ (a point).

2. Changing any map to an inclusion and to a projection (up to homotopy type): axiom 1 of $\S 1$.

3. Exactness of the functors $\operatorname{Hom}^{*}(X$,$) and \operatorname{Hom}^{*}(, X)$.

4. The tensor product $X \otimes Y=X \times Y / X \vee Y$.

5. The definition, for a pair $X, Y \in S$, of $X \otimes_{Z} Y$, given multiplications $X \otimes Z \rightarrow$ $X$ and $Z \otimes Y \rightarrow Y$.

6. Existence of a "point"-pair $P=\left(S^{0}, *\right)$ such that $X \otimes P=X$ and $X \otimes_{p} Y=$ $X \otimes Y$.

All these operations are carried over in a natural way into the categories $S_{0}, S_{p}$, $\vec{S}, \overleftarrow{S}, \vec{S}_{p}$ and $\overleftarrow{S}_{p}$

The cohomology theory $X^{*}$ will be said to be multiplicative if there is given a multiplication

$$
X \otimes X \rightarrow X, \quad X \in \vec{S}
$$

The cohomology theory $Y^{*}$ is said to act on the right [left] of the theory $X^{*}$ if there is given a multiplication $X \otimes Y \rightarrow X$ or $Y \otimes X \rightarrow X$. 
The previously mentioned theory $P^{*}$, generated by the point spectrum $P=$ $\left(S^{0}, *\right)$, operates on all cohomology theories and is called "cohomotopy theory." Its spectrum, of course, consists of the spheres $\left(S^{n}\right)$. It is obviously multiplicative, because $P \otimes P=P$.

We now describe an interesting operation constructed on a multiplicative cohomology theory $X=\left(X_{n}\right) \in \vec{S}$ of a (not necessarily stable) spectrum of spaces.

Let $\left(H_{n}^{i}\right)$ be the spectrum of spaces of maps $H_{n}^{i}=\Omega^{n-i} X_{n}=\operatorname{Map}\left(S^{n-i}, X_{n}\right)$. Since $X$ is multiplicative and $P \otimes P=P$, we have a multiplication

$$
H_{n}^{i} \times H_{m}^{j} \rightarrow H_{m+n}^{i+j} .
$$

Let now $i=j=0$. Then

$$
H_{n}^{0} \times H_{m}^{0} \rightarrow H_{m+n}^{0} .
$$

Suppose that the cohomology ring $X^{*}(X)$ and all $X^{*}(K)$ have identities (the cohomology theory contains scalars with respect to multiplication $X \otimes X \rightarrow X$ ). Consider in the space $H_{n}^{0}$ the subspace $H_{n} \subset H_{n}^{0}=\Omega^{n} X_{n}$ which is the connected component of the element $1 \in X^{0}(P)$. We have a multiplication

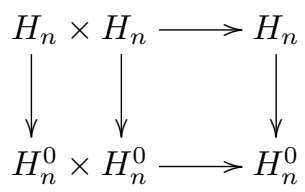

induced by the inclusion $H_{n} \subset H_{n}^{0}$.

Let $\pi(K, L)$ be the homotopy classes (ordinary, non-stable) of maps $K \rightarrow L$, and let $\Pi^{-1}(K)=\lim _{n \rightarrow \infty} \pi\left(K, H_{n}\right)$. Obviously $\Pi^{-1}(K)$ is a semigroup with respect to the previously introduced multiplication. We have

Lemma 2.1. $\Pi^{-1}(K)$ is a group, isomorphic to the multiplicative group of elements of the form $\{1+x\} \in X^{0}(K)$, where $x$ ranges over the elements of the group $X^{0}(K)$ of filtration $>0$.

The proof of Lemma 2.1 easily follows from the definition of the multiplication $H_{n} \times H_{m} \rightarrow H_{m+n}$ by means of the multiplication in the spectrum $X$.

Therefore the spectrum $\left(H_{n}\right)$ defines an " $H$-space" and the spectrum $B H=\left(B H_{n}\right)$ has often been defined. The set of homotopy classes $\pi(K, B H)=$ $\underset{n}{\lim } \pi\left(K, B H_{n}\right)$ we denote by $\Pi^{0}(K)$, while $\Pi^{0}(E K)=\Pi^{-1}(K)$ by definition, where $E$ is the suspension.

The following fact is. evident:

If $K=E^{2} L$, then $\Pi^{0}(K)=X^{1}(K)$; therefore in the $S$-category $\Pi^{0}(K)$ is simply $X^{1}(K)$. As we have already seen by Lemma 2.1 , this is not so for complexes which are only single suspensions, where $\Pi^{0}(E L)$ consists of all elements of the form $\{1+x\}$ in $X^{0}(L)$ under the multiplication in $X^{0}(L)$.

An important example. Let $X=P=\left(S^{n}, *\right)$. Then the spectrum $H_{n}$ with multiplication $H_{n} \times H_{n} \rightarrow H_{n}$ is homotopic to the spectrum $\tilde{H}_{n}$ (maps of degree +1 of $S^{n} \rightarrow S^{n}$ with composition $\left.\tilde{H}_{n} \times \tilde{H}_{n} \rightarrow \tilde{H}_{n}\right)$.

The $J$-functor of Atiyah is the image of $\bar{K}(L) \rightarrow \Pi^{0}(L)$ in our case $X=P$. In particular, in an $S$-category $L=E^{2} L^{\prime}$ we have that $\Pi^{0}(L)$ is $P^{*}(L)$; in the case $L=E L^{\prime}, \Pi^{0}(L)$ depends on the multiplication in $P^{*}\left(L^{\prime}\right)$. 
Besides the enumerated facts relating to the $S$-category of finite complexes one should also mention the existence of an anti-automorphism $\sigma: S \rightarrow S$ of this $S$ category which associates to a complex $X$ its $S$-dual complex (complement in a sphere of high dimension). The operator $\sigma$ induces

$$
\sigma: \vec{S} \rightarrow \overleftarrow{S}, \quad \sigma: \overleftarrow{S} \rightarrow \vec{S}, \quad \sigma^{2}=1
$$

Since $\operatorname{Hom}(X, Y)=\operatorname{Hom}(\sigma Y, \sigma X)$ and $\sigma X$ is a homology theory in $\overleftarrow{S}$ if $X$ is a cohomology theory, then the duality law of Alexander-Pontrjagin is, obviously, the equality $X^{*}(K)=\sigma X_{*}(\sigma K)$, and by an $X$-homology manifold is meant a complex $K$ such that $X^{i}(K)=\sigma K_{n-i}(K)$ in the presence of some natural identification of $\sigma X_{*}(K)$ with $\sigma X_{*}(\sigma K)$; for example, if $K$ is a smooth manifold, then $\sigma(K)$ according to Atiyah [6] is the spectrum of the Thom complex of the normal bundle in a sphere. In the presence of a functorial Thom isomorphism in $X^{*}$-theory for some class of manifolds, we obtain Poincaré-Atiyah duality.

Let $X \in \vec{S}, Y \in S, T \in \overleftarrow{S}$. In $\S 1$ we constructed the Adams spectral sequence with $E_{2}$ term equal to $\operatorname{Ext}_{A^{X}}^{* *}\left(X^{*}(Y), X^{*}(T)\right)$.

The law of duality for Adams spectral sequences reads:

The cohomology Adams spectral sequence $\left(E_{r}, d_{r}\right)$ with term $E_{2}=$ $\operatorname{Ext}_{A^{X}}\left(X^{*}(Y), X^{*}(T)\right)$ is canonically isomorphic to the homology Adams spectral sequence $\left(E_{r}^{\prime}, d_{r}^{\prime}\right)$ with term $E_{2}^{\prime}=\operatorname{Ext}_{A_{\sigma X}}\left(\sigma X_{*}(\sigma Y), \sigma X_{*}(\sigma T)\right)$. The homology Adams spectral sequence for $X=\sigma X=P$ was investigated by A. S. Miščenko [16].

Let us introduce the important notion of $(m-1)$-connected spectra.

Definition 2.1. The spectrum $\left(X_{n}, f_{n}\right)=X$ (direct) is called $(m-1)$-connected if each object $X_{n}$ is $\left(n+m-1+n_{0}\right)$-connected, where the integer $n_{0}$ is defined in $\S 1$. Analogously for inverse spectra.

Usually $n_{0}=0$ and $X_{n}$ is $(n+m-1)$-connected, $f_{n}: E X_{n} \rightarrow X_{n+1}$ for direct spectra. Analogously for inverse.

Finally, we should formulate two obvious facts here, which will be used later.

Lemma 2.2. a) If $X \in \vec{S}$, the cohomology theories $E X$ and $X$ have the same Adams spectral sequences for any $Y$ and $T$ for which the sequences exist (here $Y \in \vec{S}, T \in \overleftarrow{S}$.

b) Furthermore, if $\tilde{X}=\sum E^{\gamma_{i}} X$ is a direct sum, where $\gamma_{i} \rightarrow \infty$ for $i \rightarrow \infty$, then the theory $\tilde{X}^{*}$ defines the same Adams spectral sequence as the theory $X^{*}$.

Proof. Since each $\tilde{X}$-free acyclic resolution is at the same time an $X$-free resolution, the lemma at once follows from the definitions.

From the lemma follows

Corollary 2.1. For any stable Noetherian acyclic cohomology theory $X \in \vec{S}$ and any $Y \in S$ and $T \in S$, all groups $\operatorname{Ext}_{A^{X}}^{s t}\left(X^{*}(Y), X^{*}(T)\right) \otimes_{Z} Q=0$ for $s>0$.

Proof. Since a stable spectrum $X$ in the category $S_{0}=S \otimes_{Z} Q$ is equivalent to a sum $\sum E^{\gamma_{i}} K(Z)$ of Eilenberg-MacLane spectra for $\pi=Z$, and since for $X^{\prime}=K(Z)$ the $\operatorname{ring} A^{X^{\prime}} \otimes_{Z} Q$ is trivial, it follows that all $\operatorname{Ext}_{A^{X}}^{s}(,) \otimes_{Z} Q=0$ for $s>0$, since $\operatorname{Ext}_{A^{X^{\prime}}}^{s} \otimes_{Z} Q()=$,0 for $s>0$ and by virtue of Lemma 2.2 . 


\section{§ 3. IMPORTANT EXAMPLES OF COHOMOLOGY AND HOMOLOGY THEORIES. CONVERGENCE AND SOME PROPERTIES OF ADAMS SPECTRAL SEQUenCES IN COBORDISM THEORY}

We list here the majority of the most interesting cohomology theories.

1. $X=K(\pi)$, where $X_{n}=K(\pi, n)$. This theory is multiplicative if $\pi$ is a ring, and $X^{*}=H^{*}(, \pi)$. The case $\pi=Z_{p}$ is well known, having been studied in many works $[1,9,15,17,18]$. The spectral sequence was constructed by Adams in [1], where its convergence was proved $\left(\pi=Z_{p}\right)$. The ring $A^{X}$ is the usual Steenrod algebra over $Z_{p}$. Here the commonly studied case is $p=2$. The case $p>2$ was first studied in $[24] .^{3}$

The criterion (A) for the convergence of the Adams spectral sequence applies easily in the category $S_{p}=S \otimes_{Z} Q_{p}$ under the condition that $Y$ is a complex with $\pi_{i}^{*}(Y) \otimes_{Z} Q_{p}$ finite groups, in which case there is a nonacyclic resolution (the Postnikov system) which is $X$-free.

In the case $\pi=Z$, as is easy to see, the applicability of criterion (A) in the category $S$ itself again easily follows from the properties of the usual contractible spaces and Postnikov systems (see, for example, [16]).

2. Homotopy and cohomotopy theories. Let $P$ be the point in $S$, where $P_{n}=S^{n}$. The theory $P_{*}$ is that of stable homotopy groups, and $P^{*}$ that of stable cohomotopy groups. The (Eckmann-Hilton) dual of this spectrum is $K(Z)$ and the theory $H^{*}(, Z)$. Similarly, the spectra $P_{(m)}=P / m P(m$ an integer $)$ are EckmannHilton duals of the spectra $K\left(Z_{m}\right)$.

For the homology theory $P_{*}(X)$ the proof of convergence of the homology Adams spectral sequence with term $E_{2}=\operatorname{Ext}_{A_{P}}^{* *}$ is similar to the proof for the cohomology spectrum $K(Z)$ by virtue of Eckmann-Hilton duality and follows from criterion (A) of $\S 1$.

The proof of convergence for the theory $P_{(m) *}$ analogously proceeds from the method of Adams for $K\left(Z_{m}\right)$. These theories were investigated in [16].

By virtue of the law of duality for the Adams spectral sequence (cf. $\S 2$ ) and the fact that $\sigma P=P$ and $\sigma P_{(m)}=P_{(m)}$, we obtain convergence also in cohomotopy theory, where $\sigma$ is the $S$-duality operator.

3. Stable $K$-theory.

a) Let $k=\left(k_{n}\right)$, where $\Omega^{2 n} k_{2 n}=B U \times Z$, and the complexes $k_{n}$ are $(n-1)$ connected. Then $k_{2 n}$ is the $(2 n-1)$-connected space over $B U$ and the inclusion $x: k_{2 n} \rightarrow k_{2 n-2}$ is defined by virtue of Bott periodicity.

Here $k^{i}=K^{i}$ for $i \leq 0$ for $K^{*}$ the usual complex $K$-theory, and if $H^{*}(L, Z)$ has no torsion, then $k^{2 i}(L)$ is the subgroup of $K^{2 i}(L)$ consisting of elements of filtration $\geq i$.

b) Let $k O=\left(k O_{n}\right)$, where $\Omega^{8 n} k O_{8 n}=B O \times Z$, and all $k O_{n}$ are $(n-1)$-connected. We have $k O^{[i]}=\left(k O_{n}^{[i]}\right)$ where $\Omega^{8 n} k O_{8 n-i}^{[i]}=B O \times Z, k O^{[0]}=k O$ and the $k O_{n}^{[i]}$ are $(n-1)$-connected. Here $i$ is to be taken $\bmod 8$.

\footnotetext{
${ }^{3}$ In Theorem 2 of the author's work [24] there are erroneous computations, not influencing the basic results. We note also the peculiar analogues, first discovered and applied in [24], to the Steenrod powers in the cohomology of a Hopf algebra with commutative diagonal. It turns out that for all $p>2$ these "Steenrod powers" St $p^{i}$ are defined and nontrivial for $i \equiv 0,1$ $(\bmod p-1), i \geq 0$. These peculiar operations have never been noted in more recent literature on these questions, although they are of value; for example, they reflect on the multiplicative formulas of Theorem 2 in [24] for $p>2$.
} 
It is easy to show that in the category $S \otimes_{Z} Z[1 / 2]$ all spectra $k O^{[i]}$ coincide up to suspension, and the spectrum $k$ is a sum of two spectra of the type $k=$ $k O+E^{2} k O^{[2]}$.

4. Cobordism. Let $G=\left(G_{n}\right)$ be a sequence of subgroups of the groups $O_{\alpha(n)}$ where $\alpha(n+1)>\alpha(n)$ and $\alpha(n) \rightarrow \infty$ for $n \rightarrow \infty$ with $G_{n} \subset G_{n+1}$ under the inclusion $O_{\alpha(n)} \subset O_{\alpha(n+1)}$. There arise natural homomorphisms $B G_{n} \rightarrow B G_{n+1}$ and a direct spectrum (not in the $S$-category) $B G$. With this spectrum $B G$ is connected the spectrum of Thom complexes $M G=\left(M G_{n}\right)$ in the category $\vec{S}$.

Examples:

a) The spectrum $G=(e), e \subset O_{n}$; then $M G=P$;

b) $G=O, S O$, Spin, $U, S U, S p$; then $M G=M O, M S O, M$ Spin, $M U$, $M S U, M S p$ have all been investigated. All of them are multiplicative spectra and the corresponding cohomology rings have commutative multiplication with identity. Let us mention the known facts:

$$
\begin{aligned}
& \text { 1) } M O=\sum_{i} E^{\lambda_{i}} K\left(Z_{2}\right) ; \\
& \text { 2) } M S O \otimes_{Z} Q_{2}=\sum_{j} E^{\lambda_{j}} K(Z)+\sum_{q} E^{\mu_{q}} K\left(Z_{2}\right) \quad(\text { see }[17,18,23]) ; \\
& \text { 3) } M G \otimes_{Z} Q_{p}=\sum_{k} E^{\lambda_{k}} M_{(p)},
\end{aligned}
$$

where $H^{*}\left(M_{(p)}, Z_{p}\right)=A / \beta A+A \beta, A$ is the Steenrod algebra over $Z_{p}$ and $\beta$ is the $\bmod p$ Bokšteln homomorphism. This result holds for $G=S O, U$, Spin, $S p$ for $p>2, G=U$ for $p \geq 2$, and $G=S U$ for $p>2$ with reduction of the number of terms $\lambda_{k}$ corresponding to certain partitions $\omega$ (see $[15,17,18,26]$ )

$$
\text { 4) } M \operatorname{Spin} \otimes_{Z} Q_{2}=\sum_{s} E^{\lambda_{s}} K\left(Z_{2}\right)+\sum_{q} E^{\mu_{q}} k O+\sum_{l} E^{\delta_{l}} k O^{[2]} \text {. }
$$

Facts (1) and (2) are known, and fact (4) is given in a recent result of AndersonBrown-Peterson [10].

c) $G=T$, where $T^{n}=G_{n} \subset U_{n} \subset O_{2 n}$ is the maximal torus. This leads to $M G$, again a multiplicative spectrum since $M T^{m+n}=M T^{m} \otimes M T^{n}$.

Let us mention the structure of the cohomology $M_{(p)}^{*}(P)$, where $P=\left(S^{0}, *\right)$ is a point, $M_{(p)}^{*}(P)=Q_{p}\left[x_{1}, \ldots, x_{i}, \ldots\right]$ (polynomials over $\left.Q_{p}\right)$ with $\operatorname{dim} x_{i}=-2_{p^{i}}+2$ and $M_{(p)}^{0}(P)$ the scalars $Q_{p}$.

The ring $U^{*}(P)$ for $G=U$ (spectrum $\left.M U\right)$ is $Z\left[y_{1}, \ldots, y_{i}, \ldots\right]$, where $\operatorname{dim} y_{i}=$ $-2 i$.

For the spectra $M_{(p)}=X$ and $M U=X$ we have the important, simply derived

Lemma 3.1. If $a \in A^{X}$ is some operation for $X=M_{(p)} \in \vec{S} \otimes_{Z} Q_{p}$ or $X=$ $M U \in \vec{S}$ which operates trivially on the module $X^{*}(P)$, then the operation a is itself trivial.

Proof. Since $a \in \operatorname{Hom}^{*}(X, X)$, the operation $a$ is represented by a map $X \rightarrow E^{\gamma} X$. Since $\pi_{*}(X) \otimes_{Z} Q_{p}$ and $H^{*}\left(X, Q_{p}\right)$ for $X=M_{(p)}$ and $X=M U$ do not have torsion, it follows from obstruction theory in the usual fashion that the map $a: X \rightarrow E^{\gamma} X$ is completely determined by the map $a_{*}: \pi_{*}(X) \rightarrow \pi_{*}(X)$, which represents the operation $a$ on $X^{*}(P)$, for $X^{-i}(P)=\pi_{i}^{S}(X)$. End of proof of lemma. 
Since $M U \otimes_{Z} Q_{p}=\sum_{k} E^{\lambda_{k}} M(p)$, we have the following fact:

$$
\operatorname{Ext}_{A^{X}}\left(X^{*}(K), X^{*}(L)\right) \otimes_{Z} Q_{p}=\operatorname{Ext}_{A^{Y}}\left(Y^{*}(K), Y^{*}(L)\right),
$$

where $X=M U, X^{*}=U^{*}$, and $Y=M_{(p)}, Y^{*}=U_{(p)}^{*}=M_{(p)}^{*}$; we denote $M_{(p)}^{*}$ by $U_{(p)}^{*}$ and $M U^{*}$ by $U^{*}$. Both are multiplicative theories. This fact, that the Ext terms and more generally the Adams spectral sequences coincide, follows from the fact that $M U \otimes_{Z} Q_{p}=\sum_{k} E^{\lambda_{k}} M_{(p)}$, as indicated in $\S 2$, since $M U \otimes_{Z} Q_{p}$ is a sum of suspensions of a single theory $M_{(p)}$ and $Q_{p}$ is a flat $Z$-module.

For any multiplicative cohomology theory $X^{*}$ there is in the ring $A^{X}$ the operation of multiplication by the cohomology of the spectrum $P$, since the spectrum $P$ acts on every spectrum: $P \otimes X=X$. In this way there is defined a homomorphism $X^{*}(P) \rightarrow A^{X}$, where $X^{*}(P)$ acts by multiplication. From now on we denote the image of $X^{*}(P) \rightarrow A^{X}$ by $\Lambda \subset A^{X}$, the ring of "quasiscalars."

For spectra $X=M_{(p)}, X=M U$ we have the obvious

Lemma 3.2. Let $Y \in \vec{D}_{p}$ be a stable spectrum. Then $X^{*}(Y)$ is a free $\Lambda$-module, where the minimal dimension of the $\Lambda$-free generators is equal to $n$, if $Y=\left(Y_{m}\right)$ is a spectrum of $(n+m)$-connected complexes $Y_{m}$.

The lemma obviously follows from the fact that in the usual spectral sequence in which $E_{2}=H^{*}\left(Y, X^{*}(P)\right)=H^{*}(Y, \Lambda)$ for $X=M_{(p)}, M U$ all differentials $d_{r}=0$ for $r \geq 2$, and the sequence converges to $X^{*}(Y)$.

Now let $Y$ satisfy the hypotheses of Lemma 3.2. We have

Lemma 3.3. There exists an $X$-free acyclic resolution for $X=M_{(p)}, M U: Y \leftarrow$ $Y_{0} \leftarrow Y_{1} \leftarrow \cdots \leftarrow Y_{i} \leftarrow \ldots$, where the stable spectra $Y_{i} \in D_{p}$ are $(m+2 i-1)$ connected, if $Y$ is a stable $(m-1)$-connected spectrum in $\vec{D}_{p}$. Furthermore, if $X=M_{(p)}$, the spectrum $Y_{i}$ is $(m+2 i(p-1)-1)$-connected.

Proof. Since $Y$ is an $(m-1)$-connected stable spectrum, the minimal $\Lambda$-free generator of the module $X^{*}(Y)$ has dimension $m$, and the set of $m$-dimensional $\Lambda$-free generators corresponds to the generators of the group $H^{m}\left(Y, Q_{p}\right)$.

Choose in correspondence with this system of $\Lambda$-free generators an $X$-free object $C_{0}$ and construct in a natural way a map $f_{0}: Y \rightarrow C_{0}$ such that

$$
f_{0 *}: H_{m+k}\left(Y, Q_{p}\right) \rightarrow H_{m+k}\left(C_{0}, Q_{p}\right)
$$

is an isomorphism for $k \leq 1$. Obviously $C_{0}$ is also $(m-1)$-connected. Then the object $Y_{0}$ such that $0 \rightarrow Y_{0} \rightarrow Y \rightarrow C_{0} \rightarrow 0$ is a short exact sequence has the property that it is also a stable spectrum in $\vec{D}_{p}$. Furthermore, since $f_{0 *}$ is an isomorphism on the groups $H_{m+k}\left(Y, Q_{p}\right)$ for $k \leq 1$, the object $Y_{0}$ is $m$-connected in $\vec{D}_{p}$. If $X=M_{(p)}$, then it may be shown furthermore that in constructing $C_{0}$ in correspondence with $\Lambda$-free generators in $X^{*}(Y)$ the map $f_{0 *}: H_{j}\left(Y, Q_{p}\right) \rightarrow$ $H_{j}\left(C_{0}, Q_{p}\right)$ is an isomorphism for $j \leq m+2 p-3$ and a monomorphism for $j=$ $m+2 p-2$.

Therefore $Y_{0}$ will be $(m+2 p-3)$-connected if $Y$ is $(m-1)$-connected. The result for $X=M U$ in the category $D$ is obtained by substituting the minimal $p=2$. This process we continue further, and obviously obtain the desired filtration. The lemma is proved. 
Now let $T \in S$ be a finite complex. By virtue of Lemma 3.3 we have that $\operatorname{Hom}^{j}\left(T, Y_{i}\right)=0$ for large $i$. Therefore the Adams spectral sequence converges to $\operatorname{Hom}^{*}(T, Y)$ by virtue of criterion (A) in $\S 1$.

From these lemmas follows

Theorem 3.1. For any stable $(m-1)$-connected spectrum $Y \in \vec{D} \subset S, X=M U$ and any finite complex $T \in S$ of dimension $n$, the Adams spectral sequence $\left(E_{r}, d_{r}\right)$ with term $E_{2}=\operatorname{Ext}_{A^{X}}^{* *}\left(X^{*}(Y), X^{*}(T)\right)$ exists and converges exactly to $\operatorname{Hom}^{*}(T, Y)$; moreover $\operatorname{Ext}_{A^{X}}^{s, t}\left(X^{*}(Y), X^{*}(T)\right)=0$ for $t-s<s+m-n$. Furthermore, the $p$ primary part $\operatorname{Ext}_{A^{X}}^{s, t}\left(X^{*}(Y), X^{*}(T)\right) \otimes_{Z} Q_{p}=0$ for $t<2 s(p-1)+m-n$.

The proof follows immediately from the fact that if $T$ is an $n$-dimensional complex and $Y$ is a $k$-connected spectrum, then $\operatorname{Hom}_{A^{X}}^{i}\left(X^{*}(Y), X^{*}(T)\right)=0$ for $i<k-n$ and from Lemma 3.3 for $X=M U$.

The statement about the $p$-components of the groups Ext follows from Lemma 3.3 for the spectrum $M_{(p)}$, since

$$
M U \otimes_{Z} Q_{p}=\sum_{k} E^{\lambda_{k}} M(p)
$$

The theorem is proved.

Note that for $X=M U, M_{(p)}$, stable spectra $Y$ and finite complexes $T$, all groups $\operatorname{Ext}_{A^{X}}^{s, t}$ are torsion groups for $s>0$, as derived in $\S 2$.

Let $X=M_{(p)}, Y \in \vec{D}_{p}$ be a stable spectrum, and $T \in S \otimes_{Z} Q_{p}$, where the cohomology $H^{*}\left(Y, Q_{p}\right)$ and $H^{*}\left(T, Q_{p}\right)$ is different from zero only in dimensions of the form $2 k(p-1)$.

Under these hypotheses we have

Theorem 3.2. a) The groups $\operatorname{Hom}_{A^{X}}{ }^{i}\left(X^{*}(Y), X^{*}(T)\right)$ are different from zero only for $i \equiv 0 \bmod 2 p-2$;

b) $A^{X}$ is a graded ring in which elements are non-zero only in dimensions of the form $2 k(p-1)$;

c) The groups $\operatorname{Ext}_{A^{X}}^{s, t}\left(X^{*}(Y), X^{*}(T)\right)$ are different from zero only for $t \equiv 0$ $\bmod 2 p-2$;

d) In the Adams spectral sequence $\left(E_{r}, d_{r}\right)$ all differentials $d_{r}$ are equal to zero for $r \not \equiv 1 \bmod 2 p-2$.

Proof. Since the ring $X^{*}(P)$ ( $P$ a point) is nontrivial only in dimensions of the form $2 k(p-1)$, statement (b) follows from Lemma 3.1. Statement (a) follows from (b) and the hypotheses on $X^{*}(T)$. From (b) it follows that it is possible to construct an $A^{x}$-free acyclic resolution for $X^{*}(Y)$ in which generators are all of dimensions divisible by $2 p-2$. From this (c) follows. Statement (d) comes from (c) and the fact that $d_{r}\left(E_{r}^{s, t}\right) \subset E_{r}^{s+r, t+r-1}$. Q.E.D.

Corollary 3.1. For $X=M U, Y=P, T=P$ the groups $\operatorname{Ext}_{A^{X}}^{s, t}\left(X^{*}(P), X^{*}(P)\right) \otimes_{Z}$ $Q_{p}=0$ for $t<2 s(p-1)$ and for $t \not \equiv 0 \bmod 2 p-2$, and the differentials $d_{r}$ on the groups $E_{r} \otimes_{Z} Q_{p}$ are equal to zero for $r \neq 1 \bmod 2 p-2$.

From now on we always denote the cohomology $X^{*}$ for $X=M U$ by $U^{*}$ and the Steenrod ring $A^{X}$ by $A^{U}$. In the next section this ring will be completely calculated.

As for the question about the existence of the Adams spectral sequence in the theory $U^{*}$ and category $S$, we have 
Lemma 3.4. The cohomology theory $U^{*}$ is stable, Noetherian, and acyclic.

Proof. The stability of the spectrum $M U=\left(M U_{n}\right)$ is obvious. Let $T$ be a finite complex. We shall prove that $U^{*}(T)$ is finitely generated as a $\Lambda$-module, so of course as an $A^{U}$-module, where $\Lambda=U^{*}(P) \subset A^{U}$. Consider the spectral sequence $\left(E_{r}, d_{r}\right)$ with term $E_{2}=H^{*}(T, \Lambda)$, converging to $U^{*}(T)$. Since $T$ is a finite complex, in this spectral sequence only a finite number of differentials $d_{r}, \ldots, d_{k}$ are different from zero, $d_{i}=0$ for $i>k$. Note that all $d_{r}$ commute with $\Lambda$, and $E_{\infty}$ as a $\Lambda$-module is associated with $U^{*}(T)$, where $E_{\infty}=E_{k}$. The generators of the $\Lambda$-module $E_{2}$ lie in $H^{*}(T, Z) ; \Lambda^{0}=Z$ and they are finite in number: $u_{1}^{(r)}, \ldots, u_{l_{r}}^{(r)} \in E_{2}^{*, 0}$. Note that $d_{r}\left(E_{r}^{p, q}\right) \subset E_{r}^{p+r, q-r+1}$. Denote by $\Lambda_{N} \subset \Lambda$ the subring of polynomials in generators of dimension $\leq 2 N, \Lambda=U^{*}(P)=\Omega_{U}$. The ring $\Lambda_{N}$ is Noetherian. Similarly, let $\Lambda^{N} \subset \Lambda$ be the subring of polynomials in generators of dimension $>2 N$. Obviously, $\Lambda=\Lambda_{N} \otimes_{Z} \Lambda^{N}$ and $\Lambda$ has no torsion.

Assume, by induction, that the $\Lambda$-module $E_{r}$ has a finite number of $\Lambda$-generators $u_{1}^{(r)}, \ldots, u_{l_{r}}^{(r)}$ and there exists a number $N_{r}$ such that $E_{r}=\tilde{E}_{r} \otimes_{Z} \Lambda^{N_{r}}$, where $\tilde{E}_{r}$ is a $\Lambda_{N_{r}}$-module with the finite number of generators $u_{1}^{(r)}, \ldots, u_{l_{r}}^{(r)}$, above. Consider $d_{r}\left(u_{j}^{(r)}\right)=\sum_{k} \lambda_{k j}^{(r)} u_{k}^{(r)}$, where $\lambda_{k j}^{(r)} \in \Lambda$. Let $\operatorname{dim} \lambda_{k j}^{(r)} \leq \tilde{N}_{r}$ for all $k, j$. Set $N_{r+1}=$ $\operatorname{Max}\left(\tilde{N}_{r}, N_{r}\right)$. Then $\lambda_{k j}^{(r)} \in \Lambda_{N_{r+1}}$. By virtue of the Noetherian property of the ring $\Lambda_{N_{r+1}}$, the module $H\left(\tilde{E}_{r} \otimes_{Z} \Lambda_{N_{r}}^{N_{r+1}}, d_{r}\right)$ is finitely generated, where $\Lambda_{N_{r}}$ is generated by polynomial generators of dimension $N_{r}<k \leq N_{r+1}$ and $\Lambda_{N_{r}}^{N_{r+1}} \otimes_{Z} \Lambda^{N_{r+1}}=\Lambda^{N_{r}}$. Since

$$
\begin{aligned}
H\left(E_{r}, d_{r}\right) & =E_{r+1}=H\left(\tilde{E}_{r} \otimes_{Z} \Lambda^{N_{r}}, d_{r}\right)=H\left(\tilde{E}_{r} \otimes_{Z} \Lambda_{N_{r}}^{N_{r+1}} \otimes \Lambda^{N_{r+1}}, d_{r}\right) \\
& =H\left(\tilde{E}_{r} \otimes \Lambda_{N_{r}}^{N_{r+1}}, d_{r}\right) \otimes \Lambda^{N_{r+1}},
\end{aligned}
$$

if we set $\tilde{E}_{r+1}=H\left(\tilde{E}_{r} \otimes \Lambda_{N_{r}}^{N_{r+1}}, d_{r}\right)$, then $E_{r+1}$ is a finitely generated $\Lambda_{N_{r+1}}$-module, and $E_{r+1}=\tilde{E}_{r+1} \otimes_{Z} \Lambda^{N_{r+1}}$.

Taking $N_{2}=0$, we complete the induction, since for some $k, E_{k}=E_{\infty}$ is a finitely generated $\Lambda$-module. Therefore the module $U^{*}(T)$ is finitely generated and the theory $U^{*}$ is Noetherian.

Let us prove the acyclicity of the theory $U^{*}$ in the sense of $\S 1$. Since the $(4 n-2)$ skeletons $X_{2 n}$ of the complexes $M U_{n}$ do not have torsion, by virtue of the lemma for these complexes in the category $D$ the spectral sequence exists; moreover, the module $U^{*}\left(X_{2 n}\right)$ is a cyclic $A^{U}$-module with generator of dimension $2 n$ and with the single relation that all elements of filtration $\geq 2 n$ in the ring $A^{U}$ annihilate the generator. From this and the lemma it follows that $\operatorname{Ext}_{A^{U}}^{i, t}\left(U^{*}\left(X_{2 n}\right)\right)=0$ for $t<2 n-\operatorname{dim} T$, and $\operatorname{Hom}_{A U}^{*}\left(U^{*}\left(X_{2 n}\right),\right)=\operatorname{Hom}^{*}\left(, X_{2 n}\right)=\operatorname{Hom}^{*}(, X)$ in the same dimensions. From this the lemma follows easily.

Lemma 3.4 implies

Theorem 3.3. For any $Y, T \in S$ there exists an Adams spectral sequence $\left(E_{r}, d_{r}\right)$ with term $E_{r}=\operatorname{Ext}_{A^{U}}^{* *}\left(U^{*}(Y), U^{*}(T)\right)$.

A. S. Miščenko proved the convergence of this spectral sequence to $\operatorname{Hom}^{*}(T, Y)$ (see $[16])$. 


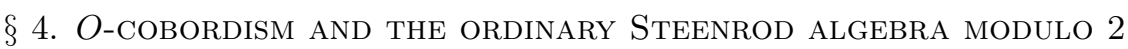

As an illustration of our method of describing the Steenrod $\operatorname{ring} A^{U}$ (see $\S \S 5$, 6 ) we exhibit it first in the simple case of the theory $O^{*}$, defined by the spectrum $M O$ isomorphic to the direct sum $M O=\sum_{\omega} E^{\lambda_{\omega}} K\left(Z_{2}\right)$, where $\omega=\left(a_{1}, \ldots, a_{s}\right)$, $\sum a_{i}=\lambda_{\omega}, a_{i} \neq 2^{j}-1$, or $\omega=0$. The Steenrod ring $A^{O}$ is an algebra over the field $Z_{2}$. Let $A$ be the ordinary Steenrod algebra. The simplest description of the algebra $A^{O}$ is the following: $A^{O}=G L(A)$ consists of infinite matrices $a=\left(a_{\omega, \omega^{\prime}}\right)$, where $\omega, \omega^{\prime}$ are nondyadic partitions $\left(a_{1}, \ldots, a_{s}\right),\left(a_{r}^{\prime}, \ldots, a_{s}^{\prime}\right), a_{\omega, \omega^{\prime}} \in A$ and $\operatorname{dim} a=\lambda_{\omega}-\lambda_{\omega^{\prime}}+\operatorname{dim} a_{\omega, \omega^{\prime}}$ is the dimension of the matrix. The $\operatorname{ring} G L(A)$ is, by definition, a graded ring. This describes the ring $A^{O}$ more generally for all spectra of the form $\sum E^{\lambda_{\omega}} K\left(Z_{2}\right)$.

In the ring $G L(A)$ we have a projection operator $\pi$ such that $\pi A^{O} \pi=A, \pi^{2}=1$, $\pi \in A^{O}=G L(A)$.

Another description of the ring $A^{O}$ is based on the existence of a multiplicative structure in $O^{*}(K, L)$. Let $\Lambda=O^{*}(P) \approx \Omega_{O}$ be the unoriented cobordism ring, $O^{i}(P)=\Omega_{O}^{i}$.

1. There is defined a multiplication operator

$$
x \rightarrow \alpha x, \quad x \in O^{*}(K, L), \quad \alpha \in \Lambda=O^{*}(P) .
$$

This defines a monomorphism $\Lambda \rightarrow A^{O}$.

2. We define "Stiefel-Whitney characteristic classes" $\tilde{W}_{i}(\xi) \in O^{i}(X)$, where $\xi$ is an $O$-bundle with base $X$ :

a) for the canonical $O_{1}$-bundle $\xi$ over $R P^{\infty}$ we set:

$$
\begin{gathered}
\tilde{W}_{i}(\xi)=0, \quad i \neq 0,1, \\
\tilde{W}_{0}(\xi)=1, \quad \tilde{W}_{1}(\xi)=D R P^{n-1} \subset O^{-1}\left(R P^{n}\right),
\end{gathered}
$$

$n$ large, $D$ the Atiyah duality operator.

b) If $\eta=\xi_{1} \oplus \xi_{2}$, then $\tilde{W}(\eta)=\tilde{W}\left(\xi_{1}\right) \tilde{W}\left(\xi_{2}\right)$, where $\tilde{W}=\sum \tilde{W}_{i}$.

These axioms uniquely define classes $\tilde{W}_{i}$ for all $O$-bundles.

As usual, the classes $\tilde{W}_{i}$ define classes $\tilde{W}_{\omega}$ for all $\omega=\left(a_{1}, \ldots, a_{s}\right)$ such that $\tilde{W}_{i}=\tilde{W}_{1, \ldots, 1}$. In $O$-theory there is defined the Thom isomorphism $\phi: O^{*}(X) \rightarrow$ $O^{*}(M \xi, *)$, where $M \xi$ is the Thom complex of $\xi$. Let $X=B O_{n}, M \xi=M O_{n}$. Let $u=\phi(1) \in O^{*}\left(M O_{n}\right)$. We define operations

$$
\mathrm{Sq}^{\omega}: O^{q}(K, L) \rightarrow O^{q+d(\omega)}(K, L)
$$

by setting $\operatorname{Sq}^{\omega}(u)=\phi\left(\tilde{W}_{\omega}\right)$, where $\tilde{W}_{\omega} \in O^{*}\left(B O_{n}\right)$.

Under the homomorphism $i^{*} \cdot j^{*}: O^{*}\left(M O_{n}\right) \rightarrow O^{*}\left(B O_{n}\right) \rightarrow O^{*}\left(\prod_{k=1}^{n} R P_{k}^{\infty}\right)$ the element $u=\phi(1)$ goes into $i^{*} j^{*}(u)=u_{1} \ldots u_{n}$, where $u_{i} \in O^{1}\left(R P_{i}^{\infty}\right)$ is the class $\tilde{W}_{1}\left(\xi_{i}\right), \xi_{i}$ the canonical $O_{1}$-bundle over $R P_{i}^{\infty}$, defined above, and $\mathrm{Sq}^{\omega}\left(u_{1} \ldots u_{n}\right)=S^{\omega}\left(u_{1}, \ldots, u_{n}\right) u_{1} \ldots u_{n}$, where $S_{\omega}$ is the symmetrized monomial $\sum u_{1}^{a_{1}} \ldots u_{s}^{a_{s}}, s \leq n$.

There is defined the subset $\operatorname{Map}\left(X, M O_{1}\right) \subset O^{1}(X)$ and a (non-additive) map $\gamma: O^{1}(X) \rightarrow H^{1}\left(X, Z_{2}\right) \rightarrow \operatorname{Map}\left(X, M O_{1}\right)$, where $\varepsilon: O^{*} \rightarrow H^{*}\left(, Z_{2}\right)$ is the natural homomorphism \{defined by the Thom class . The operations $\mathrm{Sq}^{\omega}$ have the following 
properties:

$$
\text { a) } \mathrm{Sq}^{\omega}(x y)=\sum_{\left(\omega_{1}, \omega_{2}\right)=\omega} \mathrm{Sq}^{\omega_{1}}(x) \mathrm{Sq}^{\omega_{2}}(y) \text {; }
$$

b) if $x=\gamma\left(x^{1}\right)$, then $\operatorname{Sq}^{\omega}(x)=0, \omega \neq(k)$ and $\mathrm{Sq}^{k}(x)=x^{k+1}$;

c) the composition $\mathrm{Sq}^{\omega_{1}} \circ \mathrm{Sq}^{\omega_{2}}$ is a linear combination of the form $\sum \lambda_{\omega} \mathrm{Sq}^{\omega}$, $\lambda_{\omega} \in Z_{2}$, which can be calculated on $u=\phi(1) \in O^{*}\left(M O_{n}\right)$ or on $i^{*} j^{*}(u)=$ $u_{1} \ldots u_{n} \in O^{*}\left(R P_{1}^{\infty} \times \cdots \times R P_{n}^{\infty}\right), u_{i} \in \operatorname{Im} \gamma$

d) there is an additive basis of the ring $A^{O}$ of the form $\sum \lambda_{i} \alpha_{i} \mathrm{Sq}^{\omega_{i}}, \lambda_{i} \in Z_{2}, \alpha_{i}$ an additive basis of the $\operatorname{ring} \Lambda=O^{*}(P) \approx \Omega_{O}$. Thus $A^{O}$ is a topological ring with topological basis $\alpha_{i} \mathrm{Sq}^{\omega}$, or

$$
A^{O}=(\Lambda \cdot S)^{\wedge}
$$

where $\wedge$ means completion and $S$ is the ring spanned by all $\mathrm{Sq}^{\omega}$.

We note that the set of all $\mathrm{Sq}^{\omega}$ such that $\omega=\left(a_{1}, \ldots, a_{s}\right)$, where $a_{1}=2^{j}-1$, is closed under composition and forms a subalgebra isomorphic to the Steenrod algebra $A \subset S \subset A^{O}$.

How does one compute a composition of the form $\mathrm{Sq}^{\omega} \circ \alpha$, where $\alpha \in \Lambda$ ? We shall indicate here without proof a formula for this (which will be basic in $\S 5$, where the ring $A^{U}$ is computed).

Let $(X, \xi)$ be a pair (a closed manifold and a vector bundle $\xi$ ), considered up to cobordism of pairs, i.e. $(X, \xi) \in O^{*}(B O)$. In particular, if $\xi=-\tau_{X}$, where $\tau_{X}$ is the tangent bundle, then the pair $(X, \xi) \in \Omega_{O}=O_{*}(P)$.

We define operators ("differentiations")

$$
\begin{aligned}
W_{\omega}^{*}: O^{*}(B O) & \rightarrow O^{*}(B O), \\
W_{\omega}^{*}: \Omega_{O} & \rightarrow \Omega_{O},
\end{aligned}
$$

by setting $W_{\omega}^{*}(X, \xi)=\left(Y_{\omega}, f_{\omega}^{*}\left(\xi+\tau_{X}\right)-\tau_{Y_{\omega}}\right)$, where $\left(Y_{\omega}, f_{\omega}: Y_{\omega} \rightarrow X\right)$ is $D \tilde{W}_{\omega}(\xi) \in$ $O_{*}(X)$

We also have multiplication operators

$$
\begin{aligned}
\alpha: O_{*}(B O) & \rightarrow O_{*}(B O), \\
\alpha: \Omega_{O} & \rightarrow \Omega_{O},
\end{aligned}
$$

where $(X, \xi) \rightarrow\left(X \times M, \xi \times\left(-\tau_{M}\right)\right)$ and $\left(M,-\tau_{M}\right) \in \Omega_{O}$ represents $\alpha \in \Omega_{O}$.

In particular, we have the formula

$$
W_{\omega}^{*} \cdot \alpha=\sum_{\omega=\left(\omega_{1}, \omega_{2}\right)} W_{\omega_{1}}^{*}(\alpha) \cdot W_{\omega_{2}}^{*}
$$

where $\alpha \in \Omega_{O}, W_{\omega_{1}}^{*}(\alpha) \in \Omega_{O}$.

It turns out that the following formula holds:

$$
\mathrm{Sq}^{\omega} \cdot \alpha=\sum_{\omega=\left(\omega_{1}, \omega_{2}\right)} W_{\omega_{1}}^{*}(\alpha) \cdot \mathrm{Sq}^{\omega_{2}}
$$

where $\alpha \in \Lambda=\Omega_{O}$.

We also have a diagonal

$$
\Delta: A^{O} \rightarrow A^{O} \otimes_{\Omega_{O}} A^{O},
$$

where $\Delta(\alpha)=\alpha \otimes 1=1 \otimes \alpha$, and $\Delta \mathrm{Sq}^{\omega}=\sum_{\omega=\left(\omega_{1}, \omega_{2}\right)} \mathrm{Sq}^{\omega_{1}} \otimes \mathrm{Sq}^{\omega_{2}}$, so that $A^{O} \otimes_{\Omega_{O}} A^{O}$ may be considered as an $A^{O}$-module via $\Delta ; A^{O} \otimes_{\Omega_{O}} A^{O}=O^{*}(M O \otimes M O)$, and $\Delta$ arises from the multiplication in the spectrum, $M O \otimes M O \rightarrow M O$. 
We note that the homomorphisms $W_{\omega}^{*}$ coincide with the Stiefel characteristic residues if $n=\operatorname{dim} \omega$.

We also note that any characteristic class $h \in O^{*}(B O)$ defines an operation $h \in$ $A^{O}$, if we set $h(u)=\phi(h)$, where $u \in O^{*}(M O)$ is the Thom class and $\phi: O^{*}(B O) \rightarrow$ $O^{*}(M O)$ is the Thom isomorphism.

In particular we consider the operations

$$
\begin{aligned}
& \partial(u)=\varphi\left(h_{1}\right), \quad \text { where } h_{1}=\gamma\left(\tilde{W}_{1}\right), \\
& \Delta(u)=\varphi\left(h_{2}\right), \quad \text { where } h_{2}=\gamma\left(\tilde{W}_{1}\right)^{2} .
\end{aligned}
$$

It turns out that $\partial^{2}=0, \Delta \partial=0$ and the condition $h_{1}(\xi)=0$ defines an $S O$-bundle, since $h_{1}=\gamma\left(\tilde{W}_{1}\right)$.

Further, it turns out that $O^{*}(M S O)$ is a cyclic $A^{O}$-module with a single generator $v \in O^{*}(M S O)$, given by the relations $\partial(v)=0, \Delta(v)=0$, and we have a resolution

$$
\left(\cdots \rightarrow C_{i} \rightarrow \ldots \stackrel{d}{\rightarrow} C_{1} \stackrel{d}{\rightarrow} C_{0} \stackrel{\varepsilon}{\rightarrow} O^{*}(M S O) \rightarrow O\right)=C
$$

where $C_{0}=A^{O}$ (generator $\left.u_{0}\right), C_{i}=A^{O}+A^{O}$ (generators $u_{i}, v_{i}, i \geq 1$ ), and

$$
\begin{gathered}
d\left(u_{i}\right)=\partial u_{i-1}, \quad i \geq 1, \\
d\left(v_{i}\right)=\Delta u_{i-1} .
\end{gathered}
$$

The homomorphisms $\partial^{*}$ and $\Delta^{*}: \Omega_{O} \rightarrow \Omega_{O}$ coincide with the homomorphisms of Rohlin [20], [21] and Wall [23].

We consider the complex $\operatorname{Hom}_{A^{O}}^{*}\left(C, O^{*}(P)\right)$ with differential $d^{*}$ defined by the operators $\partial^{*}$ and $\Delta^{*}$ on $O^{*}(P) \approx \Omega$. The homology of this complex is naturally isomorphic to $\operatorname{Ext}_{A^{O}}^{* *}\left(O^{*}(M S O), O^{*}(P)\right)$ or the $E_{2}$ term of the Adams spectral sequence.

It is possible to prove the following:

1) all Adams differentials are zero;

2) $\operatorname{Ext}_{A^{O}}^{0, *}\left(O^{*}(M S O), O^{*}(P)\right)=\Omega_{S O} / 2 \Omega_{S O} \subset \Omega_{O}$ where $\Omega_{S O} / 2 \Omega_{S O}=\operatorname{Ker} \partial^{*} \cap$ $\operatorname{Ker} \Delta^{*}$ by definition of the complex $C$;

3) $\mathrm{Ext}_{A^{O}}^{i, i+s}=0$, for $s \neq 4 k$;

4) $\operatorname{Ext}_{A^{O}}^{i, i+4 k}\left(O^{*}(M S O), O^{*}(P)\right)$ is isomorphic to $Z_{2}+\cdots+Z_{2}$, where the number of summands is equal to the number of partitions of $k$ into positive summands $\left(k_{1}, \ldots, k_{s}\right), \sum k_{i}=k$;

$5)$ there exists an element $h_{0} \in \operatorname{Ext}_{A^{O}}^{1,1}$ associated with multiplication by 2 in $E_{\infty}$, such that $\operatorname{Ext}_{A^{O}}^{0, t} \stackrel{h_{0}}{\longrightarrow} \operatorname{Ext}_{A^{O}}^{1, t+1}$ is an epimorphism, $t=4 k$, and $\mathrm{Ext}_{A}^{i, t} \stackrel{h_{0}}{\longrightarrow} \operatorname{Ext}_{A}^{i+1, t+1}$ is an isomorphism, $i \geq 1$.

These facts actually are trivial since

$$
\operatorname{Ext}_{A^{O}}\left(O^{*}(X), O(Y)\right)=\operatorname{Ext}_{A}\left(H^{*}\left(X, Z_{2}\right), H^{*}\left(Y, Z_{2}\right)\right)
$$

and $H^{*}\left(M S O, Z_{2}\right)$, as was shown by the author [17, 18] and by Wall [23], is $H^{*}\left(\sum E^{j} K\left(Z_{2}\right)\right)+H^{*}\left(\sum E^{k} K(Z)\right)$, where there are as many summands of the form $K(Z)$ as would be necessary for (4) and (5).

We have mentioned these facts here in connection with the analogy later of $M S O$ with $M S U$ and the paper of Conner and Floyd [13].

In the study of $\operatorname{Ext}_{A^{U}}\left(U^{*}(M S U)\right)$ all dimensions will be doubled, the groups $E_{\infty}^{i, 8 k+i}$ for $1 \leq i<3$ will be constructed in an identical fashion, but the element 
$h_{0} \in \operatorname{Ext}^{1,1}$ will be replaced by an element $h \in \operatorname{Ext}_{A^{U}}^{1,2}$ and the Adams differential $d_{3}$ will be non-trivial (see $\S \S 6,7$ ).

We note that the construction described here gives us a natural representation of the ring $A^{O}=(\Lambda \circ S)^{\Lambda}$ on the ring $\Omega_{O}$ by means of the operators $W_{\omega}^{*}$ ("differentiation") and the multiplication on $\Lambda$.

In a certain sense the operators $W_{\omega}^{*}$ generalize the ordinary characteristic numbers. They can be calculated easily for $\left[R P^{2 h}\right] \in \Omega_{O}$ and

$$
W_{\omega}^{*}(\alpha \beta)=\sum_{\left(\omega_{1}, \omega_{2}\right)=\omega} W_{\omega_{1}}^{*}(\alpha) W_{\omega_{2}}(\beta)
$$

(the Leibnitz formula). Completing their calculation would require that they be known also for "Dold manifolds."

It is interesting that the ring $A \subset A^{O}$, where $A \subset S$, is also represented monomorphically by the representation $W_{\omega}^{*}$ on $\Omega_{O}$.

In conclusion, we note that the lack of rigor in this section is explained by the fact that $O^{*}$-theory will not be considered later and all assertions will be established in the more difficult situation of $U^{*}$-theory.

\section{$\S 5$. Cohomology operations in the theory of $U$-COBordism}

In this section we shall give the complete calculation of the ring $A^{U}$ of cohomology operations in $U^{*}$-cohomology theory. We recall that for any smooth quasicomplex manifold (possibly with boundary) there is the Poincaré-Atiyah duality law

$$
U^{i}(X)=U_{n-i}(X, \partial X) \quad \text { and } \quad U_{i}(X)=U^{n-i}(X, \partial X),
$$

where quasicomplex means a complex structure in the stable tangent (or normal) bundle. Here there is also the Thom isomorphism $\phi: U^{i}(X) \rightarrow U^{2 n+i}(M \xi$, *) where $\xi$ is a complex $U_{n}$-bundle of dimension $2 n$, and $M \xi$ is its Thom complex. We denote the Poincaré-Atiyah duality operator by $D$. There is defined a natural homomorphism $\varepsilon: U_{*}(X) \rightarrow U_{*}(P)$, where $P$ is a point and $\Omega_{U}=U_{*}(P)=Z\left[x_{1}, \ldots, x_{i}, \ldots\right]$, $\operatorname{dim} x_{i}=2 i$.

We consider the group $U_{*}(K)$ given by pairs $(X, f)$, where $X$ is a manifold and $f: X \rightarrow K$. Let $\alpha$ be arbitrary characteristic class, $\alpha \in U^{*}(B U)$. For any complex $K$ in the category $S$, the class $\alpha$ defines an operator

$$
\alpha: U_{*}(K) \rightarrow U_{*}(K),
$$

if we set $\alpha(X, f)=\left(Y_{\alpha}, f \cdot f_{\alpha}\right)$, where $\left(Y_{\alpha}, f_{\alpha}\right) \in U_{*}(X)$ is the element having the form $D \alpha\left(-\tau_{X}\right)$, where $\tau_{X} \in K(X)$ is the stable tangent $U$-bundle of $X$. $\left\{D_{\alpha}\left(-\tau_{X}\right)=D\left(\left(-\tau_{X}\right)^{*}(\alpha)\right)\right\}$

As we know, the operation of the class $\alpha$ on $U_{*}(K)$ can be defined in another way: since $U^{*}(M U)=U^{*}(B U)$ by virtue of the Thom isomorphism $\phi$, we have $\phi(\alpha)=a \in U^{*}(M U)=A^{U}$. We consider the pair $L=(K \cup P, P)$ in the $S$ category; then $U_{*}(K)=\operatorname{Hom}^{*}(P, M U \otimes L)$ by definition, where $P$ is the spectrum of a point. Every operation $a=\phi(\alpha)$ defines a morphism $\phi(\alpha): M U \rightarrow M U$ and, of course, a morphism

$$
\varphi(\alpha) \otimes 1: M U \otimes L \rightarrow M U \otimes L .
$$

Hence there is defined a homomorphism $\bar{\alpha}^{*}: U_{*}(K) \rightarrow U_{*}(K)$ by means of $\phi(\alpha) \otimes 1$. We have the simple 
Lemma 5.1. The operators $\alpha^{*}$ and $\bar{\alpha}^{*}$ coincide on $U_{*}(K)$.

The proof of this lemma follows easily from the usual considerations with Thom complexes, connected with $t$-regularity.

Thus there arises a natural representation of the $\operatorname{ring} A^{U}$ on $U_{*}(K)$ for any $K$, where $a \rightarrow\left[\phi^{-1}(a)\right]^{*}=\alpha^{*}, \phi: U^{*}(B U) \rightarrow U^{*}(M U)$.

We have

Lemma 5.2. For $K=P$, the representation $a \rightarrow\left[\phi^{-1}(a)\right]^{*}$ of the Steenrod ring $A^{U}$ in the ring of endomorphisms of $U_{*}(P)=\Omega_{U}$ is dual by Poincaré-Atiyah duality to the operation of the ring $A^{U}$ on $U^{*}(P)$ and is a faithful representation.

Proof. Since $K=P$ and $M U \otimes P=M U$, the operation of the $\operatorname{ring} A^{U}$ on $\operatorname{Hom}^{*}(P, M U)$ is dual to the ordinary operation, by definition. By virtue of Lemma 3.1 of $\S 3$, this operation is a faithful representation of the $\operatorname{ring} A^{U}$. The lemma is proved.

We now consider the operation of the ring $A^{U}$ on $U_{*}(P)$ and extend it to another operation on $U_{*}(B U)$. Let $x \in U_{*}(B U)$ be represented by the pair $(X, \xi), \xi \in$ $K^{0}(X)$. We set

$$
\tilde{a}(x)=\tilde{a}(X, \xi)=\left(Y_{\alpha}, f_{\alpha}^{*}\left(\xi+\tau_{X}\right)-\tau_{Y_{\alpha}}\right),
$$

where $\alpha=\phi^{-1}(a), a \in U^{*}(M U)$ and $\left(Y_{\alpha}, f_{\alpha}\right)$ is the element of $U_{*}(X)$ equal to $D \alpha(\xi), \alpha \in U_{*}(B U)$, and $\tau_{M}$ is the stable tangent $U$-bundle of $M$.

If $\xi=\tau_{X}$, then $f_{\alpha}^{*}\left(\xi+\tau_{X}\right)-\tau_{Y_{\alpha}}=-\tau_{Y_{\alpha}}$ and hence the pair $\left(X,-\tau_{X}\right)$ goes to $\left(Y_{\alpha},-\tau_{Y_{\alpha}}\right)$, i.e., the subgroup $U_{*}(P) \subset U_{*}(B U)$ is invariant under the transformation $\tilde{a}$.

We have the obvious

Lemma 5.3. The representation $a \rightarrow \tilde{a}$ of the ring $A^{U}$ on $U_{*}(B U)$ is well-defined and is faithful.

Proof. The independence of the definition of $\tilde{a}$ from the choice of representative $(X, \xi)$ of the class $x$ follows from the standard arguments verifying invariance with respect to cobordism of pairs $(X, \xi)$ and properties of Poincaré-Atiyah duality for manifolds with boundary.

The fidelity of the representation $\tilde{a}$ follows from the fact that it is already faithful on $U_{*}(P) \subset U_{*}(B U)$ by the preceding lemma, where $\tilde{a}$ coincides with $\left[\phi^{-1}(a)\right]^{*}$. The only thing that remains to be verified is that $\tilde{a}$ is a representation of the ring $A^{U}$ and not of some extension of it. For this however, we note that the composition of transformations $\tilde{a} \tilde{b}$ is also induced by some characteristic class and hence has the form $\tilde{a} \tilde{b}=\tilde{c}$. Whence follows the lemma.

Remark 5.1. It is easy to show that the transformation $\tilde{a}$ has the form $\phi^{-1} a_{*} \phi$, where $\phi: U_{*}(B U) \rightarrow U_{*}(M U)$ and $a_{*}: U_{*}(M U) \rightarrow U_{*}(M U)$ is the transformation induced by $a: M U \rightarrow M U$. In the future we shall use the geometric meaning of the transformation $\tilde{a}=\phi^{-1} a_{*} \phi$ and hence we have given the definition of $\tilde{a}$ in a geometric form.

The transformation $\tilde{a}$ induces a transformation $\alpha^{*}: \Omega_{U} \rightarrow \Omega_{U}=U_{*}(P)$, where $U_{i}(P)=\Omega_{U}^{i}=U^{-i}(P)$.

We shall also denote by $\alpha^{*}$ the dual transformation $U^{*}(P) \rightarrow U^{*}(P), U^{*}(P)=$ $\Lambda \approx \Omega_{U}$. 
We shall now indicate the set of operations needed, from which we can construct all the operations of the Steenrod ring $A^{U}$.

1. Multiplication operators. For any element $a \in U^{*}(P)=\Lambda$ there is defined the multiplication operator $x \rightarrow a x$. Hence $\Lambda \subset A^{U}$. The corresponding transformation $\tilde{a}: U_{*}(B U) \rightarrow U_{*}(B U)$ has the form:

$$
(X, \xi) \rightarrow\left(X \times Y_{a}, \xi \times\left(-\tau_{Y_{a}}\right)\right)
$$

where $\left(Y_{a},-\tau_{Y_{a}}\right)$ represents the element $D a \in U_{*}(P)=\Omega_{U}$.

2. Chern classes and their corresponding cohomology operations. As Conner and Floyd remarked in [11], if in the axioms for the ordinary Chern classes one replaces the fact that $c_{1}(\xi)$ for the canonical $U_{1}$-bundle over $C P^{N}$ is the homology class dual to $C P^{N-1}$, by the fact that the "first Chern class" $\sigma_{1}(\xi)$ is the canonical cobordism class $\sigma_{1} \in U^{2}\left(C P^{N}\right)$ which is dual, by Atiyah, to $\left[C P^{N-1}\right]$, then there arise classes $\sigma_{i}(\xi) \in U^{2 i}(X)$ with the following properties:

1. $\sigma_{i}=0, i<0 ; \sigma_{0}=1 ; \sigma_{i}=0, i>\operatorname{dim}_{C} \xi$;

2. $\sigma_{i}(\xi+\eta)=\sum_{j+k=i} \sigma_{i}(\xi) \sigma_{k}(\eta)$;

3. $\sigma_{1}(\xi) \in \operatorname{Map}(X, M U) \subset U^{2}(X)$, if $\xi$ is a $U_{1}$-bundle;

4. $\nu\left(\sigma_{i}\right)=c_{i}$, where $\nu: U^{*} \rightarrow H^{*}(, Z)$ is the map defined by the Thom class.

We note that in the usual way (by the symbolic generators of Wu) the characteristic classes $\sigma_{i}$ determine classes $\sigma_{\omega}(\xi), \omega=\left(k_{1}, \ldots, k_{s}\right)$, such that $\sigma_{\omega}(\xi+\eta)=$

$$
\sum_{\left(\omega_{1}, \omega_{2}\right)} \sigma_{\omega_{1}}(\xi) \sigma_{\omega_{2}}(\eta) \text {, with } \sigma_{(1, \ldots, 1)}=\sigma_{i} .
$$

In the usual way the classes $\sigma_{\omega}$ determine elements $S_{\omega}=\phi \sigma_{\omega} \in U^{*}(M U)$ and, as was shown earlier, homomorphisms $\sigma_{\omega}^{*}: \Omega_{U} \rightarrow \Omega_{U}$ and $\tilde{S}_{\omega}: U_{*}(B U) \rightarrow U_{*}(B U)$.

We have the important

Lemma 5.4. The following commutation formula is valid:

$$
S_{\omega} \cdot x=\sum_{\omega=\left(\omega_{1}, \omega_{2}\right)} \sigma_{\omega_{1}}^{*}(x) S_{\omega_{2}}, \quad x \in \Lambda=U^{*}(P) \subset A^{U} .
$$

Proof. This formula can be established easily for the operation on $U_{*}(B U)$ by the faithful reprentation which we constructed earlier. Let $(X, \xi)$ represent an element of $U_{*}(B U)$ and $\left(M,-\tau_{M}\right)$ represent an element $x$ of $\Omega_{U}$. We consider

$$
\begin{aligned}
\tilde{S}_{\omega} \circ \tilde{x}(X, \xi) & =\tilde{S}_{\omega}\left[(X, \xi) \times\left(M,-\tau_{M}\right)\right]=\sum_{\omega=\left(\omega_{1}, \omega_{2}\right)} \sigma_{\omega_{1}}^{*}(x) \tilde{\sigma}_{\omega_{2}}(X, \xi) \\
& =\sum_{\omega=\left(\omega_{1}, \omega_{2}\right)}\left(Y_{\omega_{1}}, f_{\omega_{1}}^{*}\left(\xi+\tau_{X}\right)-\tau_{Y_{\omega_{1}}}\right) \times\left(N_{\omega_{2}}-\tau_{N_{\omega_{2}}}\right)
\end{aligned}
$$

by definition. Here $\left(Y_{\omega_{1}}, f_{\omega_{1}}\right)$ represents the element $D \sigma_{\omega_{1}}(\xi)$, and similarly for $N_{\omega_{2}}$. The lemma is proved.

In order that the formula derived above be more effective, we shall indicate exactly the action of the operator $\sigma_{\omega}^{*}$ on the ring $\Omega_{U}$.

It is known that by virtue of the Whitney formula the classes $\sigma_{\omega}(-\xi)$ are linear forms in the classes $\sigma_{\omega}(\xi)$ with coefficients which are independent of $\xi$. Let $\bar{\sigma}_{\omega}(\xi)=$ $\sigma_{\omega}(-\xi)$ and let $\bar{\sigma}_{\omega}^{*}$ be the homomorphism associated with this linear form. 
If $\left(X,-\tau_{X}\right)$ represents an element $a$ of $\Omega_{U}$, then the classes $\bar{\sigma}_{\omega}^{*}(a)$, represented by $\varepsilon D \bar{\sigma}_{\omega}\left(-\tau_{X}\right) \in \Omega_{U}\{$ where $\varepsilon$ is induced by $X \rightarrow P\}$ are the characteristic classes of the tangent bundle.

Let $X=C P^{n}$ and $u_{\omega}=\sum_{i_{j} \leq n+1} t_{i_{1}}^{k_{1}} \ldots t_{i_{s}}^{k_{s}}$ (the sum over all symmetrizations, $\omega=$ $\left.\left(k_{1}, \ldots, k_{s}\right)\right)$. Let $\lambda_{\omega}$ be the number of summands in the symmetrized monomial $u_{\omega}, k=\sum k_{i}$. We have the simple

Lemma 5.5. If $X=\left[C P^{n}\right]$, then $\bar{\sigma}_{\omega}^{*}(X)=\lambda_{\omega}\left[C P^{n-k}\right]$ and

$$
\bar{\sigma}_{\omega}^{*}(a b)=\sum_{\omega=\left(\omega_{1}, \omega_{2}\right)} \bar{\sigma}_{\omega_{1}}^{*}(a) \bar{\sigma}_{\omega_{2}}^{*}(b), \quad a, b, \in \Omega_{U} .
$$

Hence the above formula completely determines the action of the operators $\sigma_{\omega}^{*}$ and $\bar{\sigma}_{\omega}^{*}$ on the ring $\Omega_{U}$.

Proof. Since for $X=\left[C P^{n}\right]$ we have that $\tau_{X}+1=(n+1) \xi$, where $\xi$ is the canonical $U_{1}$-bundle, the $\mathrm{Wu}$ generators for $\tau_{X}$ are $u=t_{1}=\cdots=t_{n+1}=D C P^{n-1} \in U^{2}(X)$. Therefore $\bar{\sigma}_{\omega}^{*}\left[C P^{n}\right]=\lambda_{\omega} u^{k}$, where $k=\operatorname{dim} \omega$.

We note that by virtue of the structure of the intersection ring $U_{*}\left(C P^{n}\right)$ we have: $u^{k}=D C P^{n-k}$. Hence

$$
\varepsilon D \bar{\sigma}_{\omega}^{*}[X]=\varepsilon \lambda_{\omega} C P^{n-k}=\lambda_{\omega}\left[C P^{n-k}\right] \in \Omega_{U}
$$

\{where $\varepsilon: U_{*}\left(C P^{n}\right) \rightarrow U_{*}(P)$ is the augmentation\}. The Leibnitz formula for $\bar{\sigma}_{\omega}^{*}(a b)$ follows in the usual way from the Whitney formula. The lemma is proved.

We shall now describe the structure of the ring $S$ generated by the operators $S_{\omega}$. We consider the natural inclusions

$$
C P_{1}^{\infty} \times \cdots \times C P_{n}^{\infty} \stackrel{i}{\rightarrow} B U_{n} \stackrel{j}{\rightarrow} M U_{n}
$$

and homomorphisms

$$
\begin{gathered}
j^{*}: U^{*}\left(M U_{n}\right) \rightarrow U^{*}\left(B U_{n}\right), \\
i^{*}: U^{*}\left(B U_{n}\right) \rightarrow U^{*}\left(C P_{1}^{\infty} \times \cdots \times C P_{n}^{\infty}\right) .
\end{gathered}
$$

We note that $U^{*}\left(C P_{1}^{\infty} \times \cdots \times C P_{n}^{\infty}\right)$ has generators $u_{i} \in U^{2}\left(C P_{i}^{\infty}\right)$, and an additive basis of $U^{*}\left(C P_{1}^{\infty} \times \cdots \times C P_{n}^{\infty}\right)$ has the form $\sum \lambda_{q} x_{q} P_{q}\left(u_{1}, \ldots, u_{n}\right)$, where $x_{q} \in \Lambda=U^{*}(P)$, the $\lambda_{q}$ are integers and $P_{q}$ are polynomials. We have the following facts:

1. The image $\operatorname{Im} i^{*}$ consists of all sums of the form $\sum \lambda_{q} x_{q} P_{q}\left(u_{1}, \ldots, u_{n}\right)$, where $P_{q}$ is a symmetric polynomial and $\operatorname{dim} x_{i} P_{i}=$ constant (the series is taken in the graded ring).

2. The image $\operatorname{Im}\left(i^{*} j^{*}\right)$ consists of the principal ideal in $\operatorname{Im} i^{*}$ generated by the element $u_{1} \ldots u_{n}$.

3. The $i^{*} \sigma_{q}=\sigma_{q}\left(u_{1}, \ldots, u_{n}\right)$ are the elementary symmetric polynomials, $\sigma_{q}$ the characteristic classes.

4. For any $a \in U^{*}\left(B U_{n}\right)$ we have the usual formula $i^{*}(a)\left(u_{1} \ldots u_{n}\right)=i^{*} j^{*} \phi(a)$, where $\phi$ is the Thom isomorphism.

From these facts easily follows

Lemma 5.6. The operations $S_{\omega} \in A^{U}$ have the following properties:

1. If $\alpha \in \operatorname{Map}\left(X, M U_{1}\right) \subset U^{2}(X)$, then $S_{(k)} \alpha=\alpha^{k+1}$ and $S_{\omega}(\alpha)=0$ if $\omega \neq(k)$. 
2. $S_{\omega}(\alpha, \beta)=\sum_{\omega=\left(\omega_{1}, \omega_{2}\right)} S_{\omega_{1}}(\alpha) S_{\omega_{2}}(\beta)$ for all $\alpha, \beta \in U^{*}$.

3. If $k^{(i)}<n, \omega_{i}=\left(k_{1}^{(i)}, \ldots, k_{s_{i}}^{(i)}\right), \sum_{j} k_{j}^{(i)}=k^{(i)}$ and $a=\sum \lambda_{i} S_{\omega_{i}}$, then $a \phi(1)=a u=0$ is equivalent to $a=0$.

4. The composition of operations $S_{\omega_{1}} \cdot S_{\omega_{2}}$ is a linear combination of operations of the form $S_{\omega}$ with integral coefficients, so that an additive basis for the ring $S$ consists of all $S_{\omega}$.

Proof. Let $X=B U_{1}=C P^{\infty}$. Since $M U_{1}=C P^{\infty}$, it is sufficient to prove property 1 for the element $u \in U^{2}\left(C P^{\infty}\right)$ equal to $\sigma_{1}(\xi)$ for the canonical $U_{1}$-bundle $\xi$. By definition, we have: $u=j^{*} \phi(1) \in U^{2}\left(C P^{\infty}\right)$ and $S_{\omega}(u)=j^{*} S_{\omega} \phi(1)=j^{*} u^{k+1}$ (if $\omega=(k)$ ) and $\sigma_{\omega}(\xi)=0$, if $\omega \neq(k)$, since $\sigma_{i}=0, i \geq 2$, for $U_{1}$-bundles $\xi$. This proves property 1 .

Property 2 follows obviously from the Whitney formula for the classes $\sigma_{\omega}$ together with the remark that $\phi(1) \in U^{*}\left(M U_{n}\right)$ as $n \rightarrow \infty$ represents the universal element corresponding to the operation $1 \in A^{U}$.

Property 3 is clear. Property 4 follows from the fact that on the basis of properties 1 and 2 it is possible to compute completely $S_{\omega_{1}} \cdot S_{\omega_{2}}(u)=\sum \lambda_{\omega} S_{\omega}(u)$ and then use property 3 . Whence it will follow for large $n$ that $S_{\omega_{1}} \circ S_{\omega_{2}}=\sum \lambda_{\omega} S_{\omega}$.

The lemma is proved.

Further, we note the obvious circumstance: An additive topological basis of the ring $A^{U}$ has the form $x_{i} S_{\omega}$, where $x_{i}$ is an additive homogeneous basis for $U^{*}(P)$, $U^{i}(P)=\Omega_{U}$.

The topology of $A^{U}$ is defined by a filtration. This means that the finite linear combinations of the form $\sum \lambda_{i} x_{i} S_{\omega_{i}}$ are dense in $A^{U}$ and the completion coincides with $A^{U}$, which thus consists of formal series of the form $\sum \lambda_{i} x_{i} S_{\omega_{i}}$, where the $\lambda_{i}$ are integers and $\operatorname{dim} x_{i} S_{\omega_{i}}=$ constant, since $A^{U}$ is a graded ring.

Thus we have:

$$
A^{U}=(\Lambda \cdot S)^{\wedge}
$$

where the sign $\wedge$ denotes completion. Here $\Lambda=Z\left[x_{1}, \ldots, x_{i}, \ldots\right], \operatorname{dim} x_{i}=-2 i$. The ring $S$ is completely described by Lemma 5.6 , and the commutation properties by Lemmas 5.4, 5.5.

We note that $S$ is a Hopf ring with symmetric diagonal $\Delta: S \rightarrow S \times S$, where

$$
\Delta\left(S_{\omega}\right)=\sum_{\left(\omega_{1}, \omega_{2}\right)=\omega} S_{\omega_{1}} \otimes S_{\omega_{2}} .
$$

Since $M U$ is a multiplicative spectrum $M U \otimes M U \rightarrow M U$, the ring $A^{U}$ has a "diagonal"

$$
\Delta: A^{U} \rightarrow A^{U} \otimes_{\Lambda} A^{U}
$$

where $\Delta\left(S_{\omega}\right)=\sum_{\omega=\left(\omega_{1}, \omega_{2}\right)} S_{\omega_{1}} \otimes S_{\omega_{2}}$ and $x a \otimes b=a \otimes x b=x(a \otimes b)$ for $x \in \Omega_{U}=\Lambda$.

The Künneth formula for $K_{1}, K_{2} \in D$ \{complexes without torsion has the form:

$$
U^{*}\left(K_{1} \times K_{2}\right)=U^{*}\left(K_{1}\right) \otimes_{\Lambda} U^{*}\left(K_{2}\right),
$$

and hence $A^{U} \otimes A^{U}$ is an $A^{U}$-module with respect to the diagonal $\Delta$.

Moreover, we remark that $A^{U}$ has a natural representation * on the ring $\Omega_{U}$, where $\Omega_{U}^{i}=U^{-i}(P)$, under which the action of the ring $\Lambda$ goes over to the multiplication operators $\Lambda \approx \Omega_{U}$ and the $S_{\omega} \rightarrow \sigma_{\omega}^{*}$. 
We now define an important map $\gamma: U^{2} \rightarrow U^{2}$ (nonadditive), such that $\nu \gamma(x)=$ $\nu(x), \nu: U^{*} \rightarrow H^{*}(, Z)$ is defined by the Thom class, and $\gamma(x) \in \operatorname{Map}\left(X, M U_{1}\right) \subset$ $U^{2}(X)$, for $x \in U^{2}(X)$.

We consider important examples of cohomology operations related to the class $\sigma_{1}$.

1. Let $\Delta_{\left(k_{1}, k_{2}\right)} \in A^{U}$ be the cohomology operation such that

$$
\Delta_{\left(k_{1}, k_{2}\right)}=\varphi\left[\gamma\left(-\sigma_{1}\right)^{k_{1}} \gamma\left(\sigma_{1}\right)^{k_{2}}\right] \in U^{*}(M U),
$$

where $\sigma_{1} \in U^{2}(B U), \gamma: U^{2} \rightarrow U^{2}$.

In particular, $\Delta_{(1,0)}$ will be denoted by $\partial$ and $\Delta_{(1,1)}$ by $\Delta$.

We shall describe the homomorphisms $\Delta_{\left(k_{2}, k_{2}\right)}^{*}$ and $\tilde{\Delta}_{\left(k_{2}, k_{2}\right)}$ :

a) if $(X, \xi)$ represents an element of $U_{*}(B U)$ and $i_{1}: Y_{1} \rightarrow X, i_{2}: Y_{2} \rightarrow X$ are submanifolds which realize the classes $D c_{1}(\xi),-D c_{1}(\xi) \in H_{n-2}(X)$, then their normal bundles in $X$ are equal respectively to $\xi_{1}$ and $\bar{\xi}_{1}$, where $c_{1}\left(\xi_{1}\right)=-\overline{c_{1}\left(\xi_{1}\right)}=$ $-c_{1}\left(\bar{\xi}_{1}\right)=c_{1}(\xi)$.

Let

$$
Y_{k_{1}, k_{2}}=\underbrace{Y_{1} \ldots Y_{1}}_{k_{1}} \cdot \underbrace{Y_{2} \ldots Y_{2}}_{k_{2}}
$$

be the self-intersection in $U_{*}(X)$ with normal bundle

$$
i^{*}(\underbrace{\xi_{1}+\cdots+\xi_{1}}_{k_{1}}+\underbrace{\bar{\xi}_{1}+\cdots+\bar{\xi}_{1}}_{k_{2}})=W,
$$

where $i: Y_{k_{1}, k_{2}} \rightarrow X$.

We set $\tilde{\Delta}_{\left(k_{1}, k_{2}\right)}(X, \xi)=\left(Y_{\left(k_{1}, k_{2}\right)}, i^{*}(\xi+W)\right)$.

b) If $\xi=-\tau_{X}$, then the $\bar{\Delta}_{\left(k_{1}, k_{2}\right)}$ define homomorphisms $\Delta_{\left(k_{1}, k_{2}\right)}^{*}: \Omega_{U} \rightarrow \Omega_{U}$ for which the image of $\partial^{*}$ consists only of $S U$-manifolds. The operations $\partial^{*}$ and $\Delta^{*}$ on $\Omega_{U}$ were studied earlier in [13],

2. The classes and operations $\chi_{\left(k_{1}, k_{2}\right)}$. Just as was the case for the operations $\Delta_{\left(k_{1}, k_{2}\right)}$ and classes $\gamma\left(\sigma_{1}^{k_{1}}\right) \gamma\left(-\sigma_{1}\right)^{k_{2}}$, the operations $\chi_{\left(k_{1}, k_{2}\right)}$ and the classes corresponding to them will be defined for a bundle $\xi$ only as functions of $c_{1}(\xi)$ or of $\gamma\left(\sigma_{1}(\xi)\right)$. We define these classes for one-dimensional bundles $\xi$ over $C P^{n}$.

We consider the projectivization $P(\xi+k) \rightarrow C P^{n}$, where $k$ is the trivial $k$-plane bundle.

It is obvious that $\tau(P(\xi+k))=p^{*} \tau\left(C P^{n}\right)+\tau^{\prime}$, where $\tau^{\prime}$ consists of tangents to the fiber. Over $P(\xi+k)$ we have the following fibrations:

1) the Hopf fibration $\mu$ in each fiber;

2) The fibration $\xi^{\prime}=p^{*} \xi$.

It is easy to see that the stable bundle $\tau^{\prime}$ is equivalent to the sum

$$
\tau^{\prime}=\mu \bar{\xi}^{\prime}+\overbrace{\mu+\cdots+\mu}^{k \text { times }} \in K(P(\xi+k)) .
$$

We set $\left\{\right.$ here $\left.k_{1}+k_{2}=k\right\}$

$$
\tau_{\left(k_{1}, k_{2}\right)}^{\prime}=\mu \bar{\xi}^{\prime}+k_{1} \mu+k_{2} \bar{\mu}
$$

which functorially introduces a $U$-structure into the bundle $\tau_{\left(k_{1}, k_{2}\right)}^{\prime}$ such that $r \tau_{\left(k_{1}, k_{2}\right)}^{\prime}=r \tau^{\prime}$ where $r$ is the realification of a complex bundle. 
$P(\xi+k)$ has the induced $U$-structure $p^{*} \tau\left(C P^{n}\right)+\tau_{\left(k_{1}, k_{2}\right)}^{\prime}$. We denote the result by $P^{\left(k_{1}, k_{2}\right)}(\xi+k)$. We denote the pair $\left(P^{\left(k_{1}, k_{2}\right)}(\xi+k), p\right) \in U_{*}\left(C P^{n}\right)$ by $D \chi_{\left(k_{1}, k_{2}\right)}$, where $\chi_{\left(k_{1}, k_{2}\right)} \in U^{*}\left(C P^{n}\right)$.

For any fibration $\xi$ over $X$ we set $\chi_{\left(k_{1}, k_{2}\right)}(\xi)=\chi_{\left(k_{1}, k_{2}\right)}\left(\xi_{1}\right)$, where $c_{1}(\xi)=c_{1}\left(\xi_{1}\right)$ and $\xi_{1}$ is a $U_{1}$-bundle.

There arise classes $\chi_{\left(k_{1}, k_{2}\right)} \in U^{*}(B U)$, operations $\phi \chi_{\left(k_{1}, k_{2}\right)}=\Psi_{\left(k_{1}, k_{2}\right)}$, and homomorphisms $\Psi_{\left(k_{1}, k_{2}\right)}^{*}$ and $\tilde{\Psi}_{\left(k_{1}, k_{2}\right)}$.

We note that $\chi_{(0,1)}=0$. We denote the operation $\chi_{(1,0)}$ by $\chi$ and the operation $\chi_{(1,1)}$ by $\Psi$.

The homomorphism $\Psi^{*}: \Omega_{U} \rightarrow \Omega_{U}$ was studied by Conner and Floyd (see [13]).

It is easy to establish the following equations:

a) $\Delta_{\left(k_{1}, k_{2}\right)} \circ \partial=0$ (in particular, $\partial^{2}=0, \Delta \partial=0$ );

b) $\Delta \Psi=1,[\partial, \chi]=2, \chi \partial=x_{1} \circ \partial$, where $x_{1}=\left[C P^{1}\right] \in \Lambda \subset A^{U} ; \partial \Psi=0$.

We shall prove these equations. Since $\operatorname{Im} \partial^{*} \subset \Omega_{U}$ is represented by $S U$ manifolds, $\Delta_{\left(k_{1}, k_{2}\right)}^{*} \circ \partial^{*}=0$ by definition; since ${ }^{*}$ is a faithful representation of the ring $A^{U}$ by virtue of Lemma $3.1, \Delta_{\left(k_{1}, k_{2}\right)} \circ \partial=0$, where $\partial=\Delta_{(1,0)}$.

The equations $\Delta^{*} \Psi^{*}=1, \partial^{*} \Psi^{*}=0$ were proved by direct calculation in [13]. Hence $\Delta \Psi=1$ in $A^{U}$. Since $\operatorname{Im} \partial^{*}$ consists of $S U$-manifolds, it is easy to see that $\chi^{*} \partial^{*}=x_{1} \circ \partial^{*}$. This means that $\chi \partial=x_{1} \partial$. The equation $[\chi, \partial]=2$ follows easily from the fact that for one-dimensional bundles $\xi$ over $X$ such that $c_{1}(\xi)=-c_{1}(X)$, we have:

$$
c_{1}(P(\nu+1))=-2 c_{1}(\mu)=-2 c,
$$

and the class $D C$ is realized by the submanifold $X=P(\xi) \subset P(\xi+1)$.

Remark 5.2. Equations of the type $[a, b]=\lambda \circ 1$ arise frequently in the $\operatorname{ring} A^{U}$. For example, if $a_{k}=S_{k}$ and $b_{k}=\left[C P^{k}\right]$, then $\left[a_{k}, b_{k}\right]=(k+1) \circ 1$ by Lemma 5.5.

Remark 5.3. The operation $\pi=[\Delta, \Psi]=1-\Psi \Delta$ is the "projector of ConnerFloyd" $\pi^{2}=\pi$. (Conner and Floyd studied $\pi^{*}$.)

This projector has the property that it allows the complete decomposition of the cohomology theory $U^{*}$ into a sum of theories $\pi_{j} U^{*}$, where $\sum \pi_{j}=1, \pi_{j} \in A^{U}$, with $\pi_{0}=1-\Psi \Delta$ and $\pi_{j}=\Psi^{j} \Delta^{j}-\Psi^{j+1} \Delta^{j+1}$. Later on we shall meet other projectors of this same sort.

3. We consider still another important example of a cohomology operation in $U^{*}$-theory, connected with the following question:

Let $\xi, \eta$ be $U$-bundles. How does one compute the class $\sigma_{1}(\xi \otimes \eta)$ ?

We have

Lemma 5.7. a) For any $U_{n}$-bundle $\xi$ there is a cohomology operation $\gamma_{n-1} \in A^{U}$ such that $\sigma_{1}\left(\lambda_{-1}(\xi)\right)=\gamma_{n-1}\left(\sigma_{n}(\xi)\right)$, where $\lambda_{-1}=\sum(-1)^{i} \Lambda^{i}$ and the $\Lambda^{i}$ are the exterior powers.

b) If $u_{1}, \ldots, u_{n} \in U^{2}(X)$ are elements in the subset $\operatorname{Im} \gamma=\operatorname{Map}\left(X, M U_{1}\right) \subset$ $U^{2}(X)$, then we have the equation

$$
\gamma_{n-1}\left(u_{1} \ldots u_{n}\right)=\gamma_{1}\left(u_{1} \cdot \gamma_{1}\left(u_{2} \cdot \gamma_{1}\left(\cdots \gamma_{1}\left(u_{n-1} \cdot u_{n}\right)\right) \ldots\right),\right.
$$

where $\gamma_{1}$ is such that for a pair of $U_{1}$-bundles $\xi, \eta$ we have the formula

$$
\sigma_{1}(\xi \otimes \eta)=\sigma_{1}(\xi)+\sigma_{1}(\eta)+\gamma_{1}\left(\sigma_{1}(\xi) \sigma_{1}(\eta)\right) .
$$


The proof of this lemma follows from the definition of the operation $\gamma_{1}$. Let $X=C P^{\infty} \times C P^{\infty}$ and let $\xi, \eta$ be the canonical $U_{1}$-bundles over the factors. Since $\nu \sigma_{1}(\xi \otimes \eta)=c_{1}(\xi \otimes \eta)=c_{1}(\xi)+c_{1}(\eta)$ and $\sigma_{1} \in \operatorname{Map}\left(X, M U_{1}\right)$ it is possible to calculate the class $\sigma_{1}(\xi \otimes \eta)$ completely as a function of $\sigma_{1}(\xi)$ and $\sigma_{1}(\eta)$. Namely:

$$
-\sigma_{1}(\xi)-\sigma_{1}(\eta)+\sigma_{1}(\xi \otimes \eta)=\sum_{i \geq 1, j \geq 1} x_{i, j} \sigma_{1}^{i}(\xi) \sigma_{1}^{j}(\eta), \quad x_{i, j} \in \Lambda .
$$

Since the bundle $\lambda_{1}(\xi+\eta)$ lies in a natural way in $K^{0}\left(M U_{2}\right)$ and $\lambda_{1}(\xi+\eta)=$ $\xi \otimes \eta-\xi-\eta+1$, the difference $-\sigma_{1}(\xi)-\sigma_{1}(\eta)+\sigma_{1}(\xi \otimes \eta)+1$ has the form $\gamma_{1} u$, where $u \in U^{*}\left(M U_{2}\right)$ is the fundamental class $u=\phi(1)$.

The operation $\gamma_{1}$ can be written in the form

$$
\gamma_{1} u=\sum x_{i, j} S_{(i, j)}\left(u_{1} u_{2}\right), \quad u=u_{1} u_{2},
$$

where $u_{1}=\sigma_{1}(\xi), u_{2}=\sigma_{1}(\eta)$.

Let $\omega=\left(k_{1}, \ldots, k_{s}\right)$, where $s>2$. Then $S_{\omega}(u)=0$. Hence $\gamma_{1}$ is uniquely defined $\left(\bmod x_{\omega} S_{\omega}\right)$.

We set $\gamma_{n-1}=\gamma_{1}\left(u_{1} \ldots \gamma_{1}\left(u_{n-1} u_{n}\right) \ldots\right)$ on the element $u=u_{1} \ldots u_{n}=\phi(1) \in$ $U^{*}\left(M U_{n}\right)$. The operation $\gamma_{n-1}$ is well defined $\bmod x_{\omega} S_{\omega}$, where $\omega=\left(k_{1}, \ldots, k_{s}\right)$, $s>n$. By definition, we have the formula $\sigma_{1} \lambda_{-1}(\xi)=\gamma_{n-1} \sigma_{n}(\xi)$ for a $U_{n}$-bundle $\xi$.

The lemma is proved.

Remark 5.4. It would be very useful, if it were possible, to define exactly an operation $\gamma_{1} \in A^{U} \otimes Q$ so as to satisfy the equations $\gamma_{1}^{i}=\gamma_{i}$. The meaning of this will be clarified later in $\S 8$.

We now consider analogues of the Adams operations and the Chern character in the theory of $U$-cobordism which are important for our purposes.

We have already considered above how the class $\sigma_{1}(\xi \otimes \eta)$ is related to the classes $\sigma_{1}(\xi)$ and $\sigma_{1}(\eta)$ for $U_{1}$-bundles $\xi, \eta$. Namely

$$
\sigma_{1}(\xi \otimes \eta)=u+v+\gamma_{1}(u v)
$$

where $u=\sigma_{1}(\xi), v=\sigma_{1}(\eta)$ and

$$
\gamma_{1}(u v)=\sum_{\substack{i \geq 0 \\ j \geq 0 \\ i \neq j}} x_{i j}\left(u^{i+1} v^{j+1}+u^{j+1} v^{i+1}\right)+\sum_{i \geq 0} x_{i, i} u^{i+1} v^{i+1}, \quad x_{i, j} \in \Omega_{U} .
$$

We set $u+v+\gamma_{1}(u, v)=f(u, v)$. Then we have the "law of composition" $u \oplus v=$ $f(u, v)$ for $u, v \in \operatorname{Im} \gamma_{1}=\operatorname{Map}\left(X, M U_{1}\right)$, which turns $\operatorname{Map}\left(X, M U_{1}\right)$ into a formal one-dimensional commutative group with coefficients in the graded ring $\Omega_{U}$, while $\operatorname{dim} u, v, f(u, v)=2$. As A. S. Miščenko has shown, if we make the change of variables with rational coefficients

$$
g(u)=\sum_{i \geq 0} \frac{x_{i}}{i+1} u^{i}, \quad x_{i}=\left[C P^{i}\right]
$$

where $\left[C P^{i}\right] \in \Omega_{U}^{2 i}=\Lambda^{-2 i}$, then the composition law becomes additive:

$$
g(u \oplus v)=g(f(u, v))=g(u)+g(v)
$$

(see Appendix 1). This allows the introduction of the "Chern character":

a) We set $\sigma h(\xi)=e^{g(u)}$, where $u=\sigma_{1}(\xi)$ for $U_{1}$-bundles $\xi$;

b) if $\xi=\xi_{1}+\xi_{2}$, then $\sigma h(\xi)=\sigma h(\xi)=\sigma h\left(\xi_{1}\right)+\sigma h\left(\xi_{2}\right)$;

c) if $\xi=\xi_{1} \otimes \xi_{2}$, then $\sigma h(\xi)=\sigma h\left(\xi_{1}\right) \sigma h\left(\xi_{2}\right)$. 
Thus, we have a ring homomorphism

$$
\sigma h: K(X) \rightarrow U^{*}(X) \otimes Q .
$$

We now consider an operation $a \in A^{U}$ such that

$$
\Delta a=a \otimes a \in A^{U} \otimes_{\Lambda} A^{U} .
$$

We already know some examples of such operations:

$$
\begin{aligned}
& \text { 1) } \quad a=\sum_{\omega} S_{\omega}, \\
& \text { 2) } a=\sum_{i \geq 0} S_{i \text { times }}^{(1, \ldots, 1)} .
\end{aligned}
$$

The Chern character gives a new example of such an operation $a \in A^{U} \otimes Q$ : We consider the "Riemann-Roch" transformation $\lambda_{-1}^{(n)}: U^{2 n} \rightarrow K$ which is defined by the element $\lambda_{-1}^{(n)} \in K\left(M U_{n}\right)$, and let $\lambda=\left(\lambda_{-1}^{(n)}\right), n \rightarrow \infty$. Let $\Phi^{(n)}=\sigma h^{n} \circ \lambda_{-1}^{(n)}$, and $\Phi=\left(\Phi^{(n)}\right)$. The operation $\Phi$ obviously has the property that $\Delta \Phi=\Phi \otimes \Phi$ since $\sigma h$ and $\lambda_{-1}$ are multiplicative, and if the element $\xi \in K(X)$ has filtration $m$ and the element $\eta$ has filtration $n$, then $\sigma h^{m+n}(\xi \otimes \eta)=\sigma h^{m}(\xi) \sigma h^{n}(\eta)$. It is easy to verify that the operator $\Phi$ has the following properties:

1) $\Phi^{2}=\Phi$

2) $\Phi^{*}(1)=1$,

3) $\Phi^{*}(x)=0, \operatorname{dim} x<0, x \in \Lambda, \Lambda=U^{*}(P), \Phi^{*}: \Lambda \rightarrow \Lambda$, where

$$
\Phi=\sum_{\omega} \frac{x_{i_{1}}}{i_{1}+1} \ldots \frac{x_{i_{s}}}{i_{s}+1} S_{\omega}, \quad \omega=\left(i_{1}, \ldots, i_{s}\right) .
$$

Hence, the operation $\Phi$ associated with the Chern character $\sigma h$ defines a projection operator, which selects in the theory $U^{*} \otimes Q$ the theory $H^{*}(, Q)=\Phi\left(U^{*} \otimes Q\right)$.

A multiplicative operation $a \in A^{U}$ is uniquely defined, obviously, by its value $a(u) \in U^{*}\left(C P^{\infty}\right)$, where $u \in \operatorname{Map}\left(C P^{\infty}, M U_{1}\right)$ is the canonical generator, $a(u)=$ $u(1+\ldots)$.

Conversely, the element $a(u) \in U^{*}\left(C P^{\infty}\right)$ can be chosen completely arbitrarily. For example, for $a=\sum_{\omega} S_{\omega}, a(u)=\frac{u}{1-u}$; for $a_{k}=\sum_{\omega=(k, \ldots, k)} S_{\omega}, a(u)=u\left(1+u^{k}\right)$.

For our subsequent purposes the following operations will be important:

1) The analogues of Adams operations $\Psi_{U}^{p} \in A^{U} \otimes_{Z} Z[1 / p]$.

2) Projection operators which preserve the multiplicative structure.

All these operations are given by series $a(u) \in U^{*}\left(C P^{\infty}\right)$, since $\Delta a=a \otimes a$.

We define the Adams operations $\Psi_{U}^{k}$, which arise from the requirements:

1) $\Psi_{U}^{k}(x y)=\Psi_{U}^{k}(x) \Psi_{U}(y), x, y \in U^{*}$;

2) $\Psi_{U}^{k} \cdot x=k^{i} x \cdot \Psi_{U}^{k}$, where $x \in \Lambda^{-2 i}=\Omega_{U}^{2 i}$;

3) $\Psi_{U}^{k}(u)=\frac{u \oplus \cdots \oplus u}{k}(k$ times $)$, where $u \in U^{2}\left(C P^{\infty}\right)$ is the canonical element and $\oplus$ is composition in $\operatorname{Map}\left(X, M U_{1}\right) \subset U^{2}(X)$.

Lemma 5.8. a) The series $\Psi_{U}^{k}(u)$ has the form

$$
\Psi_{U}^{k}(u)=\frac{u \oplus \cdots \oplus u}{k}=\frac{1}{k} f(u, f(u, \ldots, f(u, u \ldots)))
$$

b) $\Psi_{U}^{k *}(x)=k^{i} x, x \in \Lambda^{-2 i}=U^{-2 i}(P)=\Omega_{U}^{2 i}$;

c) $\Delta \Psi_{U}^{k}=\Psi_{U}^{k} \otimes \Psi_{U}^{k}, \Delta: A^{U} \rightarrow A^{U} \otimes_{\Lambda} A^{U}$; 
d) $\Psi_{U}^{k} \Psi_{U}^{l}=\Psi_{U}^{k l}=\Psi_{U}^{l} \Psi_{U}^{k}$

e) for a prime $p$, all $\lambda_{i}, \lambda_{i} \in \Lambda^{-2 i}$, such that

$$
\Psi_{U}^{p}(u)=u+\lambda_{2} u^{2}+\cdots+\lambda_{i} u^{i}+\ldots, \quad u \in \operatorname{Map}\left(X, M U_{1}\right),
$$

are integral for $i<p$. Hence the element $p^{n} \Psi_{U}^{p}\left(u_{1} \ldots u_{n}\right)$, where $u_{1} \ldots u_{n} \in$ $U^{2 n}\left(M U_{n}\right)$ is a universal element, is integral, and so the operation $p^{n} \Psi_{U}^{n}$ for elements of dimension $2 n$ is "integral," if the dimension of the complex is $<2 p n$. (See Appendix 2 for proof.)

4. We now consider the projection operators. The condition defining a projection operator $\pi \in A^{U}$ is obviously $\pi^{2}=\pi$, or $\pi^{* 2}=\pi^{*}$, where $\pi^{*}: \Lambda \rightarrow \Lambda$ is the natural representation. We shall consider only those $\pi$ for which $\pi(x y)=\pi(x) \pi(y)$ and $\pi(u)=\sum_{i \geq 1} \lambda_{i} u^{i} \in U^{*}\left(C P^{\infty}\right)$. Let

$$
x_{i}=\left[C P^{i}\right], \quad \pi(u)=\left(1+\sum_{i \geq 1} \lambda_{i} u^{i}\right) u,
$$

where the $\lambda_{i} \in \Lambda \otimes Q$ are polynomials in $x_{i}$ with rational coefficients, $\operatorname{dim} \lambda_{i}=-2 i$. It is easy to show that $\pi^{*}\left(\lambda_{i}\right)=0$, since $\pi^{2}=\pi$.

We shall be especially interested in the case when there exists a complete system of orthogonal projectors $\left(\pi_{j}\right), \pi_{j} \pi_{k}=0, j \neq k$, which split the cohomology theory $U^{*}$ into a direct sum of identical theories.

Let $y \in \Lambda$ and $\Delta_{y} \in A^{U} \otimes Q$ be the "operator of division by $y$," which has the following properties:

1) $\Delta_{y}(a b)=\Delta_{y}(a) b+a \Delta_{y}(b)-y \Delta_{y}(a) \Delta_{y}(b)$,

2) $\Delta_{y}^{*}(y)=1$.

Let $\Phi_{y}=y \Delta_{y}, \Psi_{y}=1-\Phi_{y} \in A^{U} \otimes Q$. It is easy to see that $\Phi_{y}^{2}=\Phi_{y}, \Psi_{y}^{2}=\Psi_{y}$, and $\Phi_{y} \circ \Psi_{y}=0$. Moreover, the collection of projectors $\pi_{i}=y^{i} \Delta_{y}^{i}-y^{i+1}$ is such that $\pi_{j} \pi_{k}=0, j \neq k$, and it decomposes the theory $U^{*} \otimes Q$ into a sum of identical theories.

Let $y_{i} \in \Lambda^{-2 j}=\Omega_{U}^{2 j}$ be a system of polynomial generators, and $\Phi_{i}=y_{i} \Delta_{y_{i}}$. We note that $\Phi_{i}^{*}\left(y_{i}\right)=0$ for $j<i$. Let $\tilde{y}_{k}=y_{k}$ for $k \leq j$ and $\tilde{y}_{k}=\left(1-\phi_{i}\right)^{*} y_{k}=\Psi_{i}^{*}\left(y_{k}\right)$. Obviously, $\Phi_{i}^{*}\left(\tilde{y}_{k}\right)=0$ for $k \neq i$ and $\Phi_{i}^{*}\left(\tilde{y}_{i}\right)=y_{i}=\tilde{y}_{i}$.

Since $\left(1-\Phi_{i}\right)^{*}\left(y_{j}\right)=y_{j}$ for $j \leq i$ and $y_{j}-y_{i} \Delta_{y_{i}}\left(y_{j}\right)=\left(1-\phi_{i}\right)^{*} y_{j}$ for $j>i$, the collection of elements $\tilde{y}_{k}$ is a system of polynomial generators.

The projectors $\pi_{j}=y_{i}^{j} \Delta_{y_{i}}^{j}-y_{i}^{j+1} \Delta_{y_{i}}^{j+1}$ clearly are such that $\pi^{*} f: \Lambda \rightarrow \Lambda$ carries monomials of the form $y_{i}^{j} \tilde{y}_{i_{1}}, \ldots, \tilde{y}_{i_{s}}, j>0$, into themselves for $i_{1}, \ldots, i_{s} \neq i$, and all other monomials into zero. This means that

$$
\operatorname{Im} \pi_{j}^{*}=y_{i}^{j} Q\left(\tilde{y}_{1}, \ldots, \hat{\tilde{y}}_{i}, \ldots\right)
$$

and

$$
\operatorname{Ker} \pi_{j}^{*}=\bigcup_{s \neq j} y^{s} Q\left(\tilde{y}_{1}, \ldots, \hat{\tilde{y}}_{i}, \ldots\right) .
$$

In particular, $1-\sum_{j} \pi_{j}$ and $\pi_{j+1}=y_{i} \pi_{j} \Delta_{y_{i}}$. Hence $\Delta_{y_{i}} \pi_{j+1}(x)=\pi_{j}\left(y_{i} x\right)$ for all $x \in U^{*}$, and all theories $\pi_{j}(U \otimes Q)$ are isomorphic.

The projector $\pi_{0}=1-y_{i} \Delta_{y_{i}}$ has the following properties:

a) $\pi_{0}(x y)=\pi_{0}(x) \pi_{0}(y)$, i.e., $\pi_{0}$ is multiplicative. 
b) The cohomology ring of a point for the theory $\pi_{0}\left(U^{*} \otimes Q\right)$ has the form $Q\left(\tilde{y}_{1}, \ldots, \hat{\tilde{y}}_{i}, \ldots\right)$, where $\pi_{0}^{*}\left(\bar{y}_{j}\right)=\bar{y}_{j}$ for $j \neq i$.

c) All theories $\pi_{s}\left(U^{*} \otimes Q\right)$ are canonically isomorphic to the theory $\pi_{0}\left(U^{*} \otimes Q\right)$ by means of the operator of multiplication by $y_{i}^{s}$, and their defining spectra differ only by suspension.

Examples of operators $\Delta_{y}$ : if $\operatorname{dim} y=2 k$, i.e., $y \in \Omega_{U}^{2 k}(P)=U^{-2 k}(P)$, and $\sigma_{(k)}^{*} y=-\lambda \neq 0$, then we set

$$
\Delta_{y}=\sum_{q \geq 1} \frac{(-1)^{q} y^{q-1}}{\lambda^{q}} S_{\underbrace{(k, \ldots, k)}_{q \text { times }}} .
$$

For the generators $y_{i} \in \Omega_{U}^{2 i}$ we have $|\lambda|=1$ for $i \neq p^{j}-1$ and $|\lambda|=p$ for $i=p^{i}-1$ for any prime $p$. Hence

$$
\Delta_{y_{i}}=\sum_{q \geq 1} y_{i}^{q-1} S_{(i, \ldots, i)} \quad\left(i \neq p^{j}-1\right)
$$

and

$$
\Delta_{y_{i}}=\sum_{q \geq 1} \frac{y_{i}^{q-1}}{p^{q}} S_{(i, \ldots, i)} \quad\left(i=p^{j}-1\right) .
$$

It is easy to see that for $i+1 \neq p^{j}$ for given $p, \Delta_{y_{i}} \in A^{U} \otimes_{Z} Q_{p}$; for $i+1=p^{j}$ and $p \geq 2, \Delta_{y_{i}} \in A^{U} \otimes Q$, where $Q_{p}$ is the $p$-adic integers.

Now let $y_{i}$ be a collection of polynomial generators of $\Omega_{U}$ and let $p$ be prime. We consider all numbers $i_{k} \neq p^{j}-1$ in the natural order, $i_{1}<i_{2}<\cdots<i_{k}<\ldots$ Let $\Phi_{k}=\left(1-y_{i_{k}} \Delta_{y_{i_{k}}}\right)$, where $k$ is some sufficiently large integer. The projector $\Phi_{k}$ is such that the ring $\Phi_{k}^{*} \Lambda \subset \Lambda$ has as a system of polynomial generators all $\tilde{y}_{i}$ for $i \neq i_{k}$, and $\Phi_{k}^{*} y_{i_{k}}=0$.

Obviously, the operator $\Phi_{k}$ commutes with the operator of multiplication by $y_{j}$ for $j \leq i_{k}$ since $\Phi_{k}=1-y_{i_{k}} \Delta_{y_{i_{k}}}$, and $\Delta_{y_{i_{k}}}$ commutes with $y_{j}, j \leq i_{k}$.

We consider the operator $\Phi_{k} \Delta_{y_{i_{k-1}}} \Phi=\tilde{\Delta}_{k-1}$. Since $\Phi_{k}$ is multiplicative, $\tilde{\Delta}_{k-1}$ is the operator of division by $\Phi_{k} y_{i_{k-1}} \Phi_{k}$. Hence in the cohomology theory $\Phi_{k}\left(U^{*}\right)$ the operator $\tilde{\Delta}_{k-1}$ has all the properties making $1-y_{i_{k-1}} \circ \tilde{\Delta}_{k-1}=\Phi_{k-1}$ a multiplicative projector, and $\Phi_{(k-1)}^{(j)}=y_{i_{k-1}}^{j} \tilde{\Delta}_{k-1}^{j}-y_{i_{k-1}}^{j+1} \tilde{\Delta}_{k-1}^{j+1}$ forms a complete system of orthogonal projectors.

Thus, $\tilde{\Phi}_{k-1}=\Phi_{k}-y_{i_{k-1}} \Phi_{k} \circ \Delta_{y_{i_{k-1}}} \circ \Phi_{k}=\Phi_{k}\left(1-y_{i_{k-1}} \Delta_{y_{i_{k-1}}}\right) \Phi_{k}$ and $\tilde{\Phi}_{k-1} \Phi_{k}=$ $\tilde{\Phi}_{k-1}$, while

$$
\tilde{\Phi}_{k-1}=\Phi_{k} \Phi_{k-1} \Phi_{k}
$$

where $\Phi_{k}=1-y_{i_{k}} \Delta_{y_{i_{k}}}$. If $\Phi_{s}=1-y_{i_{s}} \Delta_{y_{i_{s}}}$, then we set:

$$
\tilde{\Phi}_{2}^{[k]} \Phi_{1} \tilde{\Phi}_{2}^{[k]}=\tilde{\Phi}^{[k]}
$$

where $\tilde{\Phi}_{k}^{[k]}=\Phi_{k}$, or:

$$
\tilde{\Phi}_{(k-1)}^{[k]}=\tilde{\Phi}_{k}^{[k]} \Phi_{k-1} \tilde{\Phi}_{k}^{[k]}, \ldots, \tilde{\Phi}_{i}^{[k]}=\tilde{\Phi}_{i+1}^{[k]} \Phi_{i} \tilde{\Phi}_{i+1}^{[k]}, \ldots, \tilde{\Phi}^{[k]}=\tilde{\Phi}_{2}^{[k]} \Phi_{1} \tilde{\Phi}_{2}^{[k]}
$$


The projector $\Phi^{[k]}$ is obviously such that

$$
\begin{aligned}
& \text { a) } \Phi^{[k]^{*}}\left(y_{s}\right)=\left\{\begin{array}{ll}
0, & s+1 \neq p^{j}, \\
\tilde{y}_{s}, & s+1=p^{j}
\end{array} \text { for } s \leq i_{k},\right. \\
& \text { b) } \Phi^{[k]} \in A^{U} \otimes_{Z} Q_{p} .
\end{aligned}
$$

The collection of $\Phi^{[k]}$ with $k \rightarrow \infty$ is such that $\Phi^{[k] *}$ is independent of $k$ when it operates on $\Omega_{U}$ and hence the sequence $\Phi^{[k]}$ as $k \rightarrow \infty$, or the series $\sum_{k \geq 1}\left(\Phi^{[k+1]}-\right.$ $\left.\Phi^{[k]}\right)=\Phi$ defines a projector $\Phi \in A^{U} \otimes_{Z} Q_{p}$ which is multiplicative and such that:

$$
\begin{aligned}
& \text { a) } \Phi^{*}\left(y_{s}\right)= \begin{cases}0, & s \neq p^{j}-1, \\
\tilde{y}_{s}, & s=p^{j}-1,\end{cases} \\
& \text { b) } \Phi^{2}=\Phi,
\end{aligned}
$$

c) the theory $U^{*} \otimes_{Z} Q_{p}$ splits into a sum of identical theories of the form $\Phi\left(U^{*} \otimes_{Z}\right.$ $\left.Q_{p}\right)$ up to a shift of grading (suspension).

We note that the elements $\tilde{y}_{s}=\Phi^{*}\left(y_{s}\right)$ for $s=p^{j}-1$ have the property that all $\sigma_{\omega}^{*}\left(\tilde{y}_{s}\right) \equiv 0 \bmod p$ for all $\omega, \operatorname{dim} \omega=2 s$.

The cohomology theory $\Phi\left(U^{*} \otimes_{Z} Q_{p}\right)$ is given by a spectrum $M_{(p)}$, where $H^{*}\left(M_{(p)}, Z_{p}\right)=A / \beta A+A \beta, A$ the Steenrod algebra and $\beta$ the Bokšter̆n homomorphism.

Thus, we have shown

Lemma 5.9. a) There exists a multiplicative projector $\Phi \in A^{U} \otimes_{Z} Q_{p}$ such that the cohomology theory $\Phi\left(U \otimes_{Z} Q_{p}\right)$ is given by a spectrum $M_{(p)}$, where $H^{*}\left(M_{(p)}, Z_{p}\right)=$ $A / A \beta+\beta A$, and the homomorphism $\Phi^{*}: \Lambda \rightarrow \Lambda$ annihilates all polynomial generators of the ring $\Lambda=U^{*}(P) \approx \Omega_{U}$ of dimension different from $p^{j}-1$.

b) The theory $U^{*} \otimes_{Z} Q_{p}$ decomposes into a direct sum of theories of the form $M_{(p)}^{*}=U_{p}^{*}$ and their suspensions.

\section{$\S 6$. The $A^{U}$-modules of COHomology of the most important SPACES}

In this section we shall give the structure of the module $U^{*}(X)$ for the most important spectra $X=P$ (a point), $X=C P^{n}, X=R P^{2 n}, X=R P^{2 n-1}, X=$ $M S U, X=S^{2 n-1} / Z_{p}, X=B G, G=Z_{p}$.

1. Let $X=P$. The $A^{U}$-module $U^{*}(P)$ is given by one generator $u \in U^{0}(P)$ and the relations $S_{\omega}(u)=0$ for all $\omega>0$. An additive basis for $U^{*}(P)$ is given by the fact that $U^{*}(P)$ is a free one-dimensional $\Lambda$-module, where $\Lambda \approx \Omega_{U}$. We shall denote the module $U^{*}(P)$ by $\Lambda$.

Clearly, we have:

$$
\operatorname{Hom}_{A^{U}}^{*}\left(A^{U}, \Lambda\right)=U_{*}(P)=\Omega_{U} .
$$

If $d: A^{U} \rightarrow A^{U}$ is a map such that $d(1)=a \in A^{U}$, then it is easy to see that $d^{*}\left(h_{x}\right)=h_{a^{*}(x)}$, where $h_{x} \in \operatorname{Hom}_{A^{U}}\left(A^{U}, \Lambda\right), x \in \Lambda$, and $h_{x}$ is such that $h_{x}(1)=x$.

In particular, for $a=S_{\omega}$ we have $a^{*}=\sigma_{\omega}^{*}$, and for $a=\partial, \Delta$ we have $a^{*}=\partial^{*}$ or $\Delta^{*}$, the known homomorphisms of the ring $\Omega_{U}$.

These remarks are essential for computing

$$
\operatorname{Ext}_{A^{U}}\left(, U^{*}(P)\right)=\operatorname{Ext}_{A^{U}}(, \Lambda)
$$

2. Let $X=C P^{n}=\left(E^{k} C P^{n}\right) \in S$. It is easy to see that $U^{*}(X)$ is a cyclic module with generator $u \in U^{2}(X)$ satisfying the relations: 
a) $S_{\omega}(u)=0, \omega \neq(k)$,

b) $S_{(k)}(u)=0, k \geq n$.

These results are easily derived from the properties of the ring $U^{*}\left(C P^{n}\right)$ and the properties of the operations $S_{\omega}$ given in Lemma 5.6.

3. $X_{k}^{(n)}=S^{2 n+1} / Z_{k}=\left(E^{k} S^{2 n+1} / Z_{k}\right) \in S, X_{2}^{(n)}=R P^{2 n+1} . U^{*}(X)$ has two generators $u \in U^{2}\left(X_{k}^{(n)}\right), v \in U^{2 n+1}\left(X_{k}\right)$, satisfying the relations:

a) $S_{\omega}(u)=0, \omega \neq(q)$,

b) $S_{(q)}(u)=0, q \geq n$,

c) $\left(k \Psi_{U}^{k}\right)(u)=0, u \in \operatorname{Map}\left(X_{k}, M U_{1}\right)$,

d) $S_{\omega}(v)=0, \omega>0$.

These results follow from [7] for $K^{*}(B G), G=Z_{p}$, and the $\sigma_{1}: K^{0} \rightarrow U^{2}$ and the ring $U^{*}(B G)$.

4. For $X=R P^{2 n}, B G$, the module $U^{*}(X)$ is described as follows:

a) $U^{*}\left(R P^{2 n}\right)=U^{*}\left(R P^{2 n+1}\right) / v$.

b) $U^{*}\left(B Z_{k}\right)=\lim \left[U^{*}\left(X_{k}^{(n)}\right)\right]$.

5. We now consider the case $X=M S U$. Since $U^{*}(M S U)=\phi U^{*}(B S U)$ and $S U$-bundles are distinguished by the condition $c_{1}=0$, which is equivalent to the condition $\gamma \sigma_{1}=0$, we have $U^{*}(M S U)=U^{*}(M U) / \phi J\left(\gamma \sigma_{1}\right)$, where $J$ is the ideal spanned by $\left(\gamma \sigma_{1}\right), J \subset U^{*}(B U)$.

The natural map $U^{*}(M U) \rightarrow U^{*}(M S U)$ is an epimorphism. Hence $U^{*}(M S U)$ is a cyclic $A^{U}$-module with generator $u \in U^{0}(M S U)$ and $a u=0$ if and only if $a \in \phi J\left(\gamma \sigma_{1}\right)$.

In particular, $a u=0$ for $a=\Delta_{\left(k_{1}, k_{2}\right)}$.

We have the important

Theorem 6.1. a) The module $U^{*}(M S U)$ is completely described by the relations $\partial(u)=0, \Delta(u)=0$.

b) The left annihilator of the operation $\partial$ consists of all operations of the form $a \partial+b \Delta, a, b \in A^{U}$.

Proof. We consider the module $N=A^{U} / A^{U} \Delta+A^{U} \partial$ and the natural map $f: N \rightarrow$ $U^{*}(M S U)$. We shall show that this map is an isomorphism. Since for the operation $\Delta$ there exists a right inverse $\Psi$ such that $\Delta \Psi=1$ and $\partial \Psi=0$, the module $A^{U} \Delta$ is free, and it is not possible to have a relation of the form $a \Delta+b \partial$ if $a \neq 0$ or $b \partial \neq 0$.

We now consider $A^{U} \partial$. We shall establish the following facts:

1) The left annihilator of the operation $\partial$ consists precisely of the operations of the form $\phi J\left(\gamma \sigma_{1}\right) \subset A^{U}$.

2) The operations of the form $A^{U} \partial$ form a direct summand in the free abelian group of operations $A^{U}$ under addition.

We consider the representation $a \rightarrow \tilde{a}$ on $U_{*}(B U)$. Let $\xi$ be an $S U$-bundle. It is easy to see that we have the equation

$$
(X, \xi)=\tilde{\partial}\left[(X, \xi) \otimes\left(C P^{1}, \eta\right)\right],
$$

where $c_{1}(\eta)$ is the basic element of $H^{2}\left(C P^{1}\right)$. It is also obvious that $\operatorname{Im} \partial$ consists only of pairs $(X, \xi) \in U_{*}(B U)$, where $\xi$ is an $S U$-bundle. Hence $\operatorname{Im} \partial$ is precisely $U_{*}(B S U)$. Whence follows fact (1).

For the proof of (2) we note that $U_{*}(B S U)$ is a direct summand in $U_{*}(B U)$. We decompose $U^{*}(B U)$ into a direct sum $U^{*}(B U)=U^{*}(B S U)+J\left(\gamma \sigma_{1}\right)$. Then $U^{*}(M U)=A^{U}$ decomposes into a direct sum $A+B$, where $B$ is the annihilator of 
$U_{*}(B S U)$ with respect to the representation $\tilde{a}$. Obviously, $A^{U} \partial=(B+A) \partial=A \partial$. If the operation $a \in A$ is such that $a \partial$ is divisible by the integer $\lambda$, then $\tilde{a} \tilde{\partial}$ is divisible by $\lambda$, and hence for all $S U$-bundles $\xi$ the characteristic class $\phi^{-1} \bar{a}(\xi)$ is divisible by $\lambda$. Hence this class is a $\lambda$-multiple class in $U^{*}(B S U)$ and (up to $J\left(\gamma \sigma_{1}\right)$ ) a $\lambda$-multiple class in $U^{*}(B U)$. Whence follows fact (2).

We deduce from (1) and (2) that the map $f: N \rightarrow U^{*}(M S U)$ is a monomorphism. Since $N=A^{U} / A^{U} \Delta+A^{U} \partial$, it follows from (1) and (2) that the kernel Ker $f$ is a direct summand. Since $A^{U} \Delta$ is a free module and $A^{U} \partial$ is a module isomorphic to $U^{*}(M S U)$ with shifted dimension (see (1)), the equation $\operatorname{Ker} f=0$ follows from the calculation of ranks in the groups

$$
\begin{aligned}
\left(A^{U} \Delta \otimes_{\Lambda} Z\right)^{k}= & H^{(k-4)}(M U, Z), \quad\left(A^{U} \partial \otimes_{\Lambda} Z\right)^{k}=H^{k-2}(M S U, Z), \\
& \left(U^{*}(M S U) \otimes Z\right)^{k}=H^{k}(M S U, Z) .
\end{aligned}
$$

Thus, $U^{*}(M S U)=A^{U} / A^{U} \Delta+A^{U} \partial$. Since the left annihilator of the operation $\partial$ is precisely the left annihilator of the element $u \in U^{0}(M S U)$, it follows by what was proved for $U^{*}(M S U)$ that this left annihilator is precisely $A^{U} \Delta+A^{U} \partial$. The theorem is proved.

\section{$\S 7$. Calculation of the Adams spectral sequence for $U^{*}(M S U)$}

In this section we shall compute the ring

$$
\operatorname{Ext}_{A^{U}}^{* *}\left(U^{*}(M S U), \Lambda\right)
$$

and all differentials $d_{i}$ of the Adams spectral sequence $\left(E_{r}, d_{r}\right)$, where $E_{2}=\operatorname{Ext}_{A^{U}}^{* *}\left(U^{*}(M S U), \Lambda\right)$. In particular, it turns out that $d_{i}=0$ for $i \neq 3, d_{3} \neq 0$, and $E_{\infty}^{i, *}=E_{4}^{i, *}=0$ for $i \geq 3$.

For the calculation of $\operatorname{Ext}_{A^{U}}^{* *}$ we consider the complex of $A^{U}$-modules

$$
C=\left(U^{*}(M S U) \stackrel{\varepsilon}{\leftarrow} C_{0} \stackrel{d}{\longleftarrow} C_{1} \stackrel{d}{\leftarrow} \cdots \leftarrow C_{i} \ldots\right),
$$

where the generators are denoted by $u_{i} \in C_{i}$ for $i \geq 0$ and $v_{i} \in C_{i}$ for $i \geq 1$, $C_{0}=A^{U}$ and $C_{i}=A^{U}+A^{U}$ for $i \geq 1$. We set $d\left(u_{i}\right)=\partial u_{i-1}$ and $d\left(v_{i}\right)=\Delta u_{i-1}$. Since $\partial^{2}=0$ and $\Delta \partial=0, d^{2}=0$. It follows from the theorem above that $C$ is an acyclic resolution of the module $U^{*}(M S U)=H_{0}(C)$.

We now consider the complex $\operatorname{Hom}_{A^{U}}^{*}(C, \Lambda)$, where $\Lambda=U^{*}(P)$. Since $\operatorname{Hom}_{A^{U}}^{*}\left(A^{U}, \Lambda\right)=\Omega_{U}$, we obtain the complex

$$
\operatorname{Hom}_{A^{U}}^{*}(C, \Lambda)=\left(\Omega_{U} \stackrel{d^{*}}{\longrightarrow} \Omega_{U}+\Omega_{U} \stackrel{d^{*}}{\longrightarrow} \Omega_{U}+\Omega_{U} \stackrel{d^{*}}{\longrightarrow} \ldots\right),
$$

where $d^{*}=\partial^{*}+\Delta^{*}: \Omega_{U} \rightarrow \Omega_{U}+\Omega_{U}$.

Since $\Delta^{*}$ is an epimorphism, the complex $\operatorname{Hom}_{A^{U}}^{*}(C, \Lambda)$ reduces to the following:

$$
\tilde{W}=\left(W \stackrel{\partial^{*}}{\longrightarrow} W \stackrel{\partial^{*}}{\longrightarrow} W \stackrel{\partial^{*}}{\longrightarrow} \ldots\right)
$$

where $W=\operatorname{Ker} \Delta^{*} \subset \Omega_{U}$

From this we deduce the following assertion.

Lemma 7.1. a) For all $s \geq 1$, we have isomorphisms

$$
\operatorname{Ext}_{A^{U}}^{s, t}\left(U^{*}(M S U), \Lambda\right)=H_{t-2 s}\left(W, \partial^{*}\right) .
$$

b) $\operatorname{Ext}_{A^{U}}^{0, *}\left(U^{*}(M S U), \Lambda\right)=\operatorname{Ker} \partial^{*} \cap \operatorname{Ker} \Delta^{*} \subset \Omega_{U}$. 
c) If $h \in \operatorname{Ext}_{A^{U}}^{1,2}\left(U^{*}(M S U), \Lambda\right)=Z_{2}$ is the nonzero element, then the homomorphism $\alpha \rightarrow h \alpha: \operatorname{Ext}_{A^{U}}^{i, *} \stackrel{h}{\rightarrow} \operatorname{Ext}_{A^{U}}^{i+1, *}$ is an epimorphism with kernel $\operatorname{Im} \partial^{*}$ for $i=0$, and an isomorphism for $i \geq 1$ (we recall that the spectrum $M S U$ is multiplicative).

Proof. Statements (a), (b) of the lemma obviously follow from the structure of the complex $W$, in which the grading of each term is shifted by 2 from the one before by the construction. For the proof of (c), we note that $h=\frac{1}{2} \partial^{*}\left(x_{1}\right)$, where $x_{1}=\left[C P^{1}\right] \in \Omega_{U}, x_{1} \in W$, and $\partial^{*}\left(x_{1}\right)=-2$. Further, we note that $\partial^{*}\left(x_{1} y\right)=-2 y$ if $\partial^{*}(y)=0$. Hence the element $h y$ is represented by the element $\frac{1}{2} \partial^{*}\left(y x_{1}\right)$ for a representative of $y \in H^{*}\left(\tilde{W}, \partial^{*}\right)$. But since $\frac{1}{2} \partial^{*}\left(y x_{1}\right)=y$ under the condition $\partial^{*} y=0$, statement $(c)$ is proved, and therewith the lemma.

We consider the element $K=9 x_{1}^{2}-8 x_{2} \in \Omega_{U}^{4}$, where $x_{1}=\left[C P^{1}\right], x_{2}=\left[C P^{2}\right]$. Clearly, $\partial^{*} K=\Delta^{*} K=0$. The element $K$ is a generator of the group

$$
\operatorname{Ext}_{A^{U}}^{0,4}\left(U^{*}(M S U), \Lambda\right)=\operatorname{Ker} \partial^{*} \cap \operatorname{Ker} \Lambda^{*}=Z \text {. }
$$

Since $A[K]= \pm 1$, where $A=e^{-c_{1 / 2}} T$ and $T$ is the Todd genus, by virtue of the Riemann-Roch theorem there is an $i$ such that $d_{i}(K) \neq 0$ in the Adams spectral sequence, since for all 4-dimensional $S U$-manifolds the $A$-genus is even (see [20]).

It follows from dimensional considerations that $d_{2}(K)=0$ and $d_{3}(K)=h^{3}$.

We note that from dimensional considerations it follows trivially that $d_{2 k}=0$ (see theorem in $\S 2$ ). Consider the differential

$$
d_{3}: E_{3}^{p, q} \rightarrow E_{3}^{p+3, q+2}
$$

where $d_{3}(K)=h^{3}$. We have

Lemma 7.2. If $\alpha \in E_{3}^{p, q}$ for $p \geq 3$, and $d_{3}(\alpha)=0$, then $\alpha=d_{3}(\beta)$. Hence $E_{4}^{p, q}=0$ for $p \geq 3$, and $E_{\infty}=E_{4}$.

Proof. Let $d_{3}(\alpha)=0$; since $\alpha=\widetilde{h^{3} \beta}$ from Lemma 7.1, $d_{3}(\alpha)=d_{3}\left(\widetilde{h^{3} \beta}\right)=0$. Hence $\widetilde{d^{3}(\beta)}=0$ since multiplication by $h: E_{3}^{p, *} \rightarrow E_{3}^{p+1, *}$ is a monomorphism for $p>0$. This means that $\alpha=d_{3}(\widetilde{K \beta})$. Since

$$
\sum_{p \geq 3} E_{3}^{p, *}=\sum_{p \geq 3} \operatorname{Ext}_{A^{U}}^{p, *}\left(U^{*}(M S U), \Lambda\right)
$$

is the ideal generated by the element $h$, we have $E_{4}^{p, q}=0$ for $p \geq 3$.

From dimensional considerations it follows that $E_{4}^{* *}=E_{\infty}^{* *}$.

Since $E_{\infty}^{* *}=E_{\infty}^{0, *}+E_{\infty}^{1, *}+E_{\infty}^{2, *}$ is associated with $\Omega_{S U}=\pi_{*}(M S U)$, and $E_{\infty}^{1, *}=$ $h E_{\infty}^{0, *}, E_{\infty}^{2, *}=h E_{\infty}^{1, *}=h^{2} E_{\infty}^{0, *}$ we obtain

Corollary 7.1. a) $\Omega_{S U}^{2 k+1}=h \Omega_{S U}^{2 k}$; b) $h^{2} \Omega_{S U}^{2 k}=\operatorname{Tor} \Omega_{S U}^{2 k+1}$.

The equality (a) was first established in [18] by other methods, and (b) in [12].

Corollary 7.2. a) The image of $\Omega_{S U}$ in $\Omega_{U}$ is singled out within the intersection Ker $\partial^{*} \cap \operatorname{ker} \Delta^{*}$ by setting equal to zero a certain collection of linear forms mod 2, generated by the homomorphism

$$
\begin{aligned}
d_{3}:\left(\operatorname{Ker} \partial^{*} \cap \operatorname{Ker} \Delta^{*}\right)^{2 k} & =E_{3}^{0,2 k} \rightarrow E_{3}^{3,2 k+2} \\
& =h^{3}\left(\operatorname{Ker} \partial^{*} \cap \operatorname{Ker} \Delta^{*}\right)^{2 k-4}=h^{3} E_{3}^{0,2 k-4}=\sum Z_{2} .
\end{aligned}
$$


b) The group $\operatorname{Ext}_{A^{U}}^{1,2 k}=H\left(W, \partial^{*}\right)$ is isomorphic to the direct sum $\Omega_{S U}^{2 k-1}+\Omega_{S U}^{2 k-5}$, and this isomorphism comes from the differential $d_{3}$ :

$$
0 \rightarrow \Omega_{S U}^{2 k-1} \rightarrow \operatorname{Ext}_{A^{U}}^{1,2 k} \stackrel{h^{-3} d_{3}}{\longrightarrow} \Omega_{S U}^{2 k-5} \rightarrow 0,
$$

where $\operatorname{Ext}_{A^{U}}^{1,2 k}=E_{2}^{1,2 k}=E_{3}^{2 k-1} \supset \Omega_{S U}^{2 k-1}=\operatorname{Ker} d_{3}, h^{-3} d_{3}$ is well-defined since $h^{3}$ is a monomorphism on $\operatorname{Ext}_{A^{U}}^{1,2 k-4}$, and the image $\operatorname{Im} h^{-3} d_{3}=\operatorname{Ker} d_{3}=\Omega_{S U}^{2 k-5} \subset$ $\operatorname{Ext}_{A^{U}}^{1,2 k-4}$.

Corollary 7.2 follows from Lemma 7.2.

Remark 7.1. Part (b) of the corollary explains the meaning of the "Conner-Floyd exact sequence" (see [13])

$$
0 \rightarrow \Omega_{S U}^{2 k-1} \rightarrow H_{2 k-2}\left(W, \partial^{*}\right) \rightarrow \Omega_{S U}^{2 k-5} \rightarrow 0
$$

since $H_{2 k-2}\left(W, \partial^{*}\right)=\operatorname{Ext}_{A^{U}}^{1,2 k}\left(U^{*}(M S U), \Lambda\right)$.

We note now that the groups $H_{*}\left(W, \partial^{*}\right)$ are computed in [13]: namely, $H_{8 k}(W)=$ $H_{8 k+4}(W)=Z_{2}+\cdots+Z_{2}$ (the number of summands is equal to the number of partitions of the integer $k), H_{i}(W)=0, i \neq 8 k, 8 k+4$. Whence we have:

$$
\operatorname{Ext}_{A^{U}}^{1,8 k+2}\left(U^{*}(M S U), \Lambda\right)=\operatorname{Ext}_{A^{U}}^{1,8 k+6}\left(U^{*}(M S U), \Lambda\right)
$$

and

We have

$$
\operatorname{Ext}_{A^{U}}^{1, i}\left(U^{*}(M S U), \Lambda\right)=0, \quad i \neq 8 k+2,8 k+6 .
$$

Lemma 7.3. a) $\operatorname{Ext}_{A^{U}}^{1,8 k+6}=K \operatorname{Ext}_{A^{U}}^{1,8 k+2}$, where $K \in \operatorname{Ext}_{A^{U}}^{0,4}\left(U^{*}(M S U), \Lambda\right)$.

b) $d_{3}\left(\operatorname{Ext}_{A^{U}}^{0, i}\right)=0$ for $i \neq 8 k+4$, and $d_{3}\left(\operatorname{Ext}_{A^{U}}^{0,8 k+4}\right)=\operatorname{Ext}_{A^{U}}^{3,8 k+2}$ is defined by the condition $d_{3}(K)=h_{3}$.

Proof. Suppose both parts of the lemma proved for $k \leq k_{0}-1$. We show that $d_{3}\left(\operatorname{Ext}_{A^{U}}^{0,8 k_{0}}\right)=0$. In fact, by the induction hypothesis on the groups $\operatorname{Ext}_{A^{U}}^{3,8 k+2}$ the differential $d_{3}$ is a monomorphism. Hence $\operatorname{Ext}_{A^{U}}^{0,8 k_{0}} \stackrel{d_{3}}{\longrightarrow} 0$.

We now consider $d_{3}\left(K \operatorname{Ext}_{A^{U}}^{0,8 k_{0}}\right)=h^{3} \operatorname{Ext}_{A^{U}}^{0,8 k_{0}}$. We see that $d_{3}\left(K \operatorname{Ext}_{A^{U}}^{0,8 k_{0}}\right)$ is an epimorphism onto $\operatorname{Ext}_{A^{U}}^{3,8 k_{0}+6}$. Whence parts (a) and (b) of the lemma follow; on the group $\operatorname{Ext}^{3,8 k+6}$ the differential is trivial, and on the group $\operatorname{Ext}^{3,8 k+2} \supset \operatorname{Ker}_{3}=0$.

The lemma is proved.

Thus, we obtain

Corollary 7.3. a) The image $\Omega_{S U}^{i} /$ Tor $\subset \Omega_{U}$ coincides with Ker $\partial^{*} \cap \operatorname{Ker} \Delta^{*}$ for $i \neq 8 k+4$.

b) For $i=8 k+4$ the image $\Omega_{A^{U}}^{8 k+4} /$ Tor $\subset \Omega_{U}^{8 k+4}$ is picked out precisely by the requirement of the "Riemann-Roch Theorem":

$$
\operatorname{ch}(c \xi) A(X)[X] \equiv 0 \quad(\bmod 2)
$$

where $X$ is an $S U$-manifold, $\xi \in k O(X)$.

We note that (a) follows immediately from the lemma. As to (b), we note that $A[K]=1$. In [9], "Pontrjagin classes" $\pi_{l} \in k O^{*}[X]$ are introduced in $k O$ theory. Consider the classes $\pi_{2 l} \in \widetilde{k O}(X)$; let $\pi_{2 l}=\kappa_{l}$. Now consider the numbers $\operatorname{ch}\left(c \kappa_{l_{1}} \ldots c \kappa_{l_{k}}\right) A(X)[X]$ for $X \in \Omega_{U}^{8 k} /$ Tor $\subset \Omega_{U}^{8 k}$. These numbers are different from zero $\bmod 2$ if and only if $h X \neq 0$ in $\Omega_{S U}^{8 k+1}$. Hence the condition 
$d_{3}(K X)=h^{3} X \neq 0$ in $E_{3}^{3, *}$ is equivalent to the fact that one of the numbers $\operatorname{ch}\left(c\left(\kappa_{l_{1}}, \ldots, \kappa_{l_{k}}\right)\right) A(X)[X] \not \equiv 0(\bmod 2)$. All such numbers are in 1-1 correspondence with partitions of $8 k$ into summands $\left(8 l_{1}, \ldots, 8 l_{k}\right)$ (these facts are easily deduced from [9]).

Since $\operatorname{ch}\left(c \kappa_{l_{1}} \ldots c \kappa_{l_{k}} \otimes 1\right) A(X \times K)[X \times K]=\operatorname{ch}\left(c \kappa_{l_{1}} \ldots c \kappa_{l_{k}}\right) A(X)[X] \circ A[K]$, $A[K]=1$, we have found elements $\kappa_{l_{1}} \ldots \kappa_{l_{k}} \otimes 1 \in k O(X \times K)$ which do not satisfy the Riemann-Roch theorem, and they determine $\pi(k)$ linearly independent forms mod 2, where $\pi(k)$ is the number of partitions of $k$. From this part (b) of the corollary follows.

The results of the lemmas and corollaries of this section together completely describe the Adams spectral sequence for $U^{*}(M S U)$.

\section{$\S 8 . k$-THEORY IN THE CATEGORY OF COMPLEXES WITHOUT TORSION}

Here we shall consider the cohomology theory $k^{*}$, defined by the spectrum $k=$ $\left(k_{n}\right)$, where $\pi_{i}\left(k_{n}\right)=0, i<n$, and $\Omega^{2 n} k_{2 n}=B U \times Z$. The spectrum $k$ is such that the cohomology module $H^{*}\left(k, Z_{2}\right)$ is a cyclic module over the Steenrod algebra, with a generator $u \in H^{0}\left(k, Z_{2}\right)$, satisfying the relations $\operatorname{Sq}^{1}(u)=\operatorname{Sq}^{3}(u)=0$. Hence the spectrum $k$ does not lie in the category $D$ of complexes without torsion.

There is defined the "Bott operator" $x: k_{2 n} \rightarrow k_{2 n-2}$ by virtue of the Bott periodicity $\Omega^{2} k_{2 n}=k_{2 n-2}$, and $k_{2 n}$ is a connective fiber of $B U$. Since $k^{0}(X)=$ $K^{0}(X)$, we have on $k^{0}$ the Adams operations (see [2])

$$
\Psi^{k}: K^{0}(X) \rightarrow K^{0}(X),
$$

defined by morphisms $\Psi^{k}: B U \rightarrow B U$ such that $\Psi_{*}^{k}: \pi_{2 n}(B U) \rightarrow \pi_{2 n}(B U)$ is the operator of multiplication by the integer $k^{n}$ (see [2] concerning the operation of $\Psi^{k}$ on $K^{0}\left(S^{2 n}\right)=\pi_{2 n}(B U)$ ). By virtue of this, the operators $\Psi^{k}$ can be extended to the whole theory $K^{*} \otimes Q$, starting from the identity

$$
k x \Psi^{k}=\Psi^{k} x,
$$

where $x: K^{i} \rightarrow K^{i-2}$ is Bott periodicity.

In the category $D$ of complexes without torsion the operator $x^{n}: k^{2 n}(X) \rightarrow$ $k^{0}(X)$ is such that its image consists precisely of all elements in $k^{0}(X)=K^{0}(X)$ whose filtration is $\geq 2 n$; moreover, $x$ is a monomorphism.

In the category $D$ we define an operation $\left(k^{n} \Psi^{k}\right)$ by setting

$$
\left(k^{n} \Psi^{k}\right)=x^{-n} \Psi^{k} x^{n},
$$

where $\left(k^{n} \Psi^{k}\right): k^{2 n}(X) \rightarrow k^{2 n}(X)$.

It is easy to see that this is well defined and gives rise to an unstable operation $\left(k^{n} \Psi^{k}\right)$ such that $\left(k^{n} \Psi^{k}\right)$ can be considered as a map $k_{2 n} \rightarrow k_{2 n}$ for which $\left(k^{n} \Psi^{k}\right)_{*}: \pi_{2 n+2 j}\left(k_{2 n}\right) \rightarrow \pi_{2 n+2 j}\left(k_{2 n}\right)$ is multiplication by $k^{n+j}$.

Let $a_{n}=\sum_{k} \lambda_{k}^{(n)}\left(k^{n} \Psi^{k}\right)$, where the $\lambda_{k}^{(n)}$ are integers, be an unstable cohomology operation and $a_{n}^{(j)_{*}}$ multiplication by $\sum_{k} \lambda_{k}^{(n)} k^{n+j}$.

Definition 8.1. The sequence $a=\left(a_{n}\right)$ will be called a stable operation if for any $j$ there is a number $n$ such that for all $N \geq n$ the number $a_{N}^{(j)}=\sum_{k} \lambda_{k}^{(N)} k^{N+j}$ is independent of $N$. 
Definition 8.2. If the stable operation $a$ has a zero of order $q$ in the sense that $a_{*}^{(j)}=0$ for $j \leq q$, then we also call $b=\left(x^{-q} a\right)$ a stable operation, where $a=x^{q} b$, $b: k^{i}(X) \rightarrow k^{i+2 q}(X), X \in D$.

We consider the ring generated by the operations so constructed and the operation $x$ by means of composition, taking into account the facts that $k x \Psi^{k}=\Psi^{k} x$ and $\Psi^{k} \Psi^{l}=\Psi^{k l}$. The resulting uniquely defined ring, which we denote by $A_{\Psi}^{k}$, is a ring of operations acting in the category $D$. In it lies the subring of operations generated by the operations indicated in Definition 8.1 together with the periodicity $x$. This ring we denote by $B_{\Psi}^{k} \subset A_{\Psi}^{k}$. There is defined the inclusion $B_{\Psi}^{k} \rightarrow A_{\Psi}^{k}$.

We shall exhibit a basis for the $\operatorname{ring} A_{\Psi}^{k}$. It is easy to see that it is possible to construct operations $\delta_{i} \in A_{\Psi}^{k}$ of dimension $2 i$, where $\delta_{0}=1$, such that the elements $x^{k} \delta_{i}$ give an additive topological basis for the ring $A_{\Psi}^{k}$, and all elements of $A_{\Psi}^{k}$ can be described as formal series $\sum \lambda_{k} x^{k} \delta_{k-i}$, where the $\lambda_{k}$ are integers. The choice of such elements $\delta_{i}$ is of course unique only $\bmod x A_{\Psi}^{k}$ (elements of higher filtration).

We construct these elements $\delta_{i}$ in a canonical fashion: it suffices to define operations $\gamma_{i}=x_{i} \delta_{i}$ of dimension 0 . Let $\delta_{0}=1$. Let $\gamma_{1 *}^{(0)}=0$ and $\gamma_{1 *}^{(1)}$ be multiplication by 2. By definition, we shall take $\gamma_{i *}^{(j)}=0$ for $j<i$ and $\gamma_{i *}^{(i)}$ to be multiplication by a number $\tilde{\gamma}_{i}$ which is a linear combination $\tilde{\gamma}_{i}=\sum_{k} \mu_{k}^{(i)} k^{n+i}$, where the numbers $\mu_{k}^{(i)}$ are such that $\sum_{k} \mu_{k}^{(i)} k^{n+i}=0$ for $j<i$. We require in addition that $\tilde{\gamma}_{i}$ be the smallest positive integer of all linear combinations of the form $\sum_{k} \mu_{k}^{(i)} k^{n+i}$ under the conditions:

$$
\sum_{k} \mu_{k}^{(i)} k^{n+i}=0, \quad j<i .
$$

We consider the operation $a_{i n}=\sum_{k} \mu_{k}^{(i)}\left(k^{n} \Psi^{k}\right)$. Here $n$ is very large compared with $i$. It is easy to see that the number $\tilde{\gamma}_{i}$ does not depend on $n$ for large $n \rightarrow \infty$. Hence the operation is well-defined.

Consider the operations $a_{i n}$ for $n \rightarrow \infty$; we shall successively construct the $\delta_{i}$ from them. We have $a_{0 n}=1$; let $b_{m n}=a_{1 n}+\kappa_{1} a_{2 n}+\cdots+\kappa_{m} a_{m n}$ be linear combinations such that the homomorphisms $\left(b_{m n}\right)_{*}^{(j)}$ for $j \leq m \ll n$ are multiplications by integers $\tilde{\gamma}_{i, j}$, where $0 \leq \tilde{\gamma}_{1, j}<\tilde{\gamma}_{j}$. Clearly the numbers $\tilde{\gamma}_{1, j}$ are uniquely defined. Let $m \rightarrow \infty, n \rightarrow \infty$; then in the limit, the sequence $\left(b_{m n}\right)$ gives an operation which we denote by $\gamma_{1}=x_{1} \delta_{1}$. It is uniquely defined by the properties that $\gamma_{1 *}^{(0)}=0, \gamma_{1 *}^{(1)}=2$, and $0 \leq \gamma_{1 *}^{(i)}<\tilde{\gamma}_{i}, \gamma_{1 *}^{(i)}=\tilde{\gamma}_{1, i}$.

The operations $\gamma_{i}$ are constructed in a similar fashion, and are uniquely determined by the conditions $\gamma_{i *}^{(j)}=0, j<i, \gamma_{i *}^{(i)}=\tilde{\gamma}_{i}$, and $0 \leq \gamma_{i *}^{(k)}<\tilde{\gamma}_{k}$ for $k>i$.

We exhibit a table of the integers $\gamma_{i *}^{(j)}=\tilde{\gamma}_{i j}$ in low dimensions:

\begin{tabular}{c|cccc} 
& $\gamma_{0}$ & $\gamma_{1}$ & $\gamma_{2}$ & \\
\hline 0 & 1 & 0 & 0 & $\ldots$ \\
2 & 1 & 2 & 0 & $\ldots$ \\
4 & 1 & 0 & 24 & $\ldots$ \\
& $\ldots \ldots$ & $\ldots \ldots$
\end{tabular}

By definition, $\delta_{i}=x^{-i} \gamma_{i}$. It is clear that the operations $\gamma_{i}$ commute. Since $\pi_{2 i}(B U)$ is $Z$, the rings $A_{\Psi}^{k}$ and $B_{\Psi}^{k}$ are represented as operators on $k^{*}(P)=Z[x]$ in a natural 
way, in particular, the operations of dimension 0 by diagonal operators with integral characteristic values; the operation $x$ is represented by the translation operator (or multiplication by $x$ in $\left.k^{*}(P)\right)$. It is easy to show that we have a transformation $*: A_{\Psi}^{k} \rightarrow A_{\Psi}^{k}$ such that $*\left(B_{\Psi}^{k}\right) \subset B_{\Psi}^{k}$ and $a x=x a^{*}$. This transformation $*$ is completely determined by the condition that in $k^{*} \otimes Q$-theory we have $k x \Psi^{k}=\Psi^{k} x$ and ${ }^{*} \Psi^{k}=k \Psi^{k}$.

We also indicate the following simple fact.

Lemma 8.1. The greatest common divisor of the integers $\gamma_{i *}^{(q)}=\left(x^{i} \delta_{i}\right)_{*}^{(q)}$ for all $i>0$, for a fixed integer $q$, coincides precisely with the greatest common divisor of the numbers $k^{N}\left(k^{q}-1\right)$ for all $k$. There exist operations $a_{k, n} \in B_{\Psi}^{k}$ such that $a_{k, n *}^{(j)}=k^{n+j}$ for $j \leq f(n)$, where $f(n) \rightarrow \infty$ as $n \rightarrow \infty$.

The proof of this consists of the fact that the operations $x^{i} \delta_{i}=\gamma_{i}$ are obtained as linear combinations of the operations $k^{n}\left(\Psi^{k}-1\right)$ by virtue of the condition $\gamma_{i *}^{(0)}=0$ for $i>0$, where $n$ is large, and the determinant of the transition from the $k^{n}\left(\Psi^{k}-1\right)$ to the $x^{i} \delta_{i}$ is equal to 1 . In fact, the process described above for constructing $\left(x^{i} \delta_{i}\right)$ is the process of reduction of the set of transformations $k^{n}\left(\Psi^{k}-1\right)$ to the set $\gamma_{i}$ of "triangular type" on $Z[x]=k^{*}(P)$. More exactly: let $n$ be sufficiently large that $\gamma_{i, n *}^{(j)}=0$ for $j<i$ and $\gamma_{i, n *}^{(i)}=\tilde{\gamma}_{i}$ for $i<f(n)$, where $f(n) \rightarrow \infty$ as $n \rightarrow \infty$ and $\gamma_{i, n}=\sum \lambda_{k, i}^{(n)}\left(k^{n} \Psi^{k}\right)$. Under the condition $\sum \lambda_{k, i}^{(n)} k^{n}=0$, one can write all these operations in the form $\sum \mu_{k, i}^{(n)} k^{n}\left(\Psi^{k}-1\right)$ and then apply to the set $k^{n}\left(\Psi^{k}-1\right)$ the process of reduction to "triangular form" described above for constructing the operations $\left(x^{i} \delta_{i}\right)$ up to high dimensions. We assert that the passage from $\left\{k^{n}\left(\Psi^{k}-1\right)\right\}$ to $\left\{\gamma_{i, n}\right\}$ is invertible. Indeed, any operation of the form $\sum \lambda_{k} k^{n} \Psi^{k}$ has the form $\mu_{1} \gamma_{1, n}+b_{1}$, where $b_{1 *}^{(0)}=b_{1 *}^{(1)}=0$. Hence the operation $b_{1}$ has the form $b_{1}=\mu_{2} \gamma_{2, n}+b_{2}$, where $b_{2 *}^{(0)}=b_{2 *}^{(1)}=b_{2 *}^{(2)}=0$, etc.

Consequently, $a=\sum_{i \leq f(n)} \mu_{i} \gamma_{i}+b_{f(n)}$ where $b_{f(n), *}^{(j)}=0, j \leq f_{n}$. If $n \rightarrow \infty$, then $f(n) \rightarrow \infty$ and the coefficients $\mu_{i}$ stabilize, while $a_{n}=\sum \mu_{i} x^{i} \delta_{i}+b_{f(n)}$ if $a=\left(a_{n}\right) \in B_{\Psi}^{k}$. Since the greatest common divisor of the homomorphisms $a_{*}^{(j)}$, for all $a \in B_{\Psi}^{k}$ such that $a_{*}^{(0)}=0$, is invariant and this invariant can be calculated with respect to any basis of operations in $B^{k}$ such that $a_{*}^{(0)}=0$, we have that for the basis $\left(x_{i} \delta_{i}\right)=\left(\gamma_{i}\right)$ it coincides with the greatest common divisor for the basis $\left(k^{n}\left(\Psi^{k}-1\right)\right)=b_{k}$, where $b_{k *}^{(j)}=k^{n}\left(k^{j}-1\right)$. We note that the operations $\left(k^{n} \Psi^{k}\right)$ are nonstable, but, by virtue of what has been said, there exist operations $a_{k, n}$ such that $a_{k, n *}^{(j)}=k^{n+j}$ for $j \leq f(n)$, where $f(n) \rightarrow \infty$ as $n \rightarrow \infty$. These operations are obtained by the transformation from $\left(x^{i} \delta_{i}\right)$ to $\left(k^{n} \Psi^{k}\right)$ inverse to that described above.

The lemma is proved.

Remark 8.1. The same operations $\Psi^{k}$ in $k^{*} \otimes Q$ are obtained as formal sums of the form $\sum \mu_{i} x^{i} \delta_{i}=\Psi^{k}$, where $\mu_{i} \in Q$ and $k^{n} \mu_{i} \in Z$ for large $n$ and $i<f(n)$.

Example 1. Let $X=P \in D$ be the point spectrum. Then $k^{*}(P)$ has a single generator $t$ as an $A_{\Psi}^{k}$ module and is given by the relations $\delta_{i}(t)=0, i>0$. The module $k^{*}(P)$, as a $B_{\Psi}^{k}$-module, has a single generator $t$ and is given by the relations 
$\left(p^{n} \Psi^{p}\right)(t)=p^{n} t$ for all primes $p\left(n\right.$ large). (Or: all operations $a \in B_{\Psi}^{k}$ which have zeros of order one are such that $a t=0$.)

Example 2. Let $X=M U_{n}$. Then $k^{*}\left(M U_{n}\right)$ can be described by the ideal in the ring of symmetric polynomials in the $\operatorname{ring} \Lambda\left[u_{1}, \ldots, u_{n}\right], \operatorname{dim} u_{i}=2 i$, generated by $u=u_{1} \ldots u_{n}$. Let $v_{i}=x u_{i}, \Psi^{k}\left(v_{i}^{l}\right)=\left(\left(v_{i}+1\right)^{k}-1\right)^{l}$ and $\Psi^{k}(x y)=\Psi^{k}(x) \Psi^{k}(y)$. The elements of $k^{*}\left(M U_{n}\right)$ have the form $\sum \lambda_{i, s} x^{s} d_{s}$, where $d_{s}=f\left(u_{1}, \ldots, u_{n}\right)$ is an element of the symmetric ideal in $Z\left[u_{1}, \ldots, u_{n}\right]$ generated by $u=u_{1} \ldots u_{n}$, and $x$ is the Bott operator. This uniquely determines $k^{*}\left(M U_{n}\right)$ and $k^{*}(M U)$ as $A_{\Psi^{-}}^{k}$ and $B_{\Psi}^{k}$-modules.

We have the following

Lemma 8.2. The ring $B_{\Psi}^{k} \subset A_{\Psi}^{k}$ coincides exactly with the subring of $A_{\Psi}^{k}$ consisting of operations of dimension $\leq 0$.

The only thing which must be proved is that $B_{\Psi}^{k}$ contains all operations of dimension $\leq 0$. For a pair $a_{1} \in B_{\Psi}^{k}, a_{2} \in B_{\Psi}^{k}$ of operations which have zeros of order $q_{1}, q_{2}$ respectively, we introduce the operations $x^{-q_{1}} a_{1}=b_{1}$ and $x^{-q_{2}} a_{2}=b_{2}$ and the composition $b_{1} \circ b_{2}$ in $A_{\Psi}^{k}$. We shall show that $x^{q_{1}+q_{2}} b_{1} \circ b_{2}$ lies in $B_{\Psi}^{k}$ if $x^{q_{1}} b_{1} \in B_{\Psi}^{k}$. Let $a_{1 n}=\sum_{k} \lambda_{k}^{(n)} k^{n} \Psi^{k}$ and $a_{2 n}=\sum_{k} \mu_{k}^{(n)} k^{n} \Psi^{k}$. We consider

$$
\begin{aligned}
& x^{q_{1}+q_{2}} b_{1 n} \cdot b_{2 n}=x_{1}^{q_{1}+q_{2}} x^{-q_{1}} a_{1 n} x^{-q_{2}} a_{2 n} \\
& =\left(\sum_{k} \lambda_{k}^{(n)} k^{\left(n-q_{2}\right)} \Psi^{k}\right)\left(\sum_{k} \mu_{k}^{(n)} k^{n} \Psi^{k}\right)
\end{aligned}
$$

\{using $k^{q_{2}} x^{q_{2}} \Psi^{k}=\Psi^{k} x^{q_{2}}$ \}. We shall assume that $n$ is very large, $n \rightarrow \infty, q_{1}$ and $q_{2}$ are fixed. We set $m=n-q_{2}$. Then

$$
\begin{aligned}
& \left(\sum_{k} \lambda_{k}^{(n)} k^{m} \Psi^{k}\right)\left(\sum_{k} \mu_{k}^{(n)} k^{q_{2}} k^{m} \Psi^{k}\right) \\
= & \left(\sum_{k} \bar{\lambda}_{k}^{(m)} k^{m} \Psi^{k}\right)\left(\sum_{k} \bar{\mu}_{k}^{(m)} k^{m} \Psi^{k}\right),
\end{aligned}
$$

where $\lambda_{k}^{(m)}=\lambda_{k}^{(n)}$ and $\bar{\mu}_{k}^{(m)}=k^{q^{2}} \mu_{k}^{(n)}$. Clearly, as $m \rightarrow \infty$ we have a composition of operations in $B_{\Psi}^{k}$ which lies in $B_{\Psi}^{k}$.

The lemma is proved.

By virtue of the lemma, the rings $B_{\Psi}^{k}$ and $A_{\Psi}^{k}$ contain operations which coincide up to dimensions $f(n) \rightarrow \infty$ (as $n \rightarrow \infty)$ with the operations $\left(k^{n} \Psi^{k}\right)$ in the sense that $a_{k, n, *}^{(j)}=k^{n+j}$ for $j \leq(n)$.

This remark allows us to use (up to any dimension) the ring $B_{\Psi}^{k}$ as if it were the ring generated by $\left(p^{n} \Psi^{p}\right)$, with $p$ prime, and by $x \in B_{\Psi}^{k}$ where $\left(p^{n} \Psi^{p}\right) x=p x\left(p^{n} \Psi^{p}\right)$ and $\gamma_{p}=\left(p^{n} \Psi^{p}\right)$ are polynomial generators. Thus, a (topological) basis here is $x^{k} P\left(\gamma_{2}, \gamma_{3}, \ldots\right)$, where $P$ is a polynomial.

We consider the $B_{\Psi}^{k}$-module $k^{*}(P)$. We have

Lemma 8.3. The torsion part of the group $\operatorname{Ext}_{B_{\Psi}^{k}}^{1,2 i}\left(k^{*}(P), k^{*}(P)\right)$ is a cyclic group, whose order is equal to $\left\{p^{n}\left(p^{i}-1\right)\right\}_{p}$, where $n$ is large, $p$ is prime, and \{\}$_{p}$ means the greatest common divisor of the sequence of integers. 
Proof. We construct a $B_{\Psi}^{k}$-free resolution of the module $k^{*}(P)$. Let $n$ be large. Then the module $k^{*}(P)$ is given by the relations $\left(\gamma_{p}-p^{n}\right) t=0$. We choose generators $\kappa_{p}=\left(\gamma_{p}-p^{n}\right)$ and 1 . Then the $\kappa_{p}$ are polynomial elements,

$$
\begin{gathered}
\ldots \stackrel{d}{\rightarrow} C_{1} \stackrel{d}{\rightarrow} C_{0} \stackrel{\varepsilon}{\rightarrow} k^{*}(P), \\
C_{0}=B_{\Psi}^{k}, \quad C_{1}=\sum_{p} B_{\Psi, p}^{k},
\end{gathered}
$$

while $d u=t$ and $d u_{p}=\kappa_{p}(u)$, where $u, u_{p}$ are free generators of the modules $B_{\Psi}^{k}=C_{0}, B_{\Psi, p}^{k} \subset C_{1}$, respectively.

We consider the complex $\operatorname{Hom}_{B_{\Psi}^{k}}^{*}\left(C, k^{*}(P)\right)$.

Let $h_{i} \in \operatorname{Hom}_{B_{\Psi}^{k}}^{2 i}\left(C_{0}, k^{*}(P)\right)$ be elements such that $h_{i}(u)=x^{i}(t)$ and $h_{i}^{(p)} \in$ $\operatorname{Hom}^{*}\left(C_{1}, k^{*}(P)\right)$ be such that $h_{i}^{(p)}\left(u_{p}\right)=x^{i}(t)$ and $0=h_{i}^{(p)}\left(u_{p^{\prime}}\right)$ for $p^{\prime} \neq p$.

Obviously, we have

$$
\begin{aligned}
& \left(d^{*} h_{i}, u_{p}\right)=\left(h_{i}, \kappa_{p} u\right)=\kappa_{p} x^{i}(t) \\
= & p^{n}\left(\Psi^{p}-1\right) x^{i}(t)=p^{n}\left(p^{i}-1\right) x^{i}(t) .
\end{aligned}
$$

Hence, $d^{*} h_{i}=\sum_{p} p^{n}\left(p^{i}-1\right) h_{i}^{(p)}$. Thus, $\frac{d^{*}\left(h_{i}\right)}{\left\{p^{n}\left(p^{i}-1\right)\right\}_{p}}$ is a 1-cocycle for the operator $d^{*}$. Since $\operatorname{Hom}^{*}\left(, k^{*}(P)\right)$ is a free abelian group, the element $\frac{d^{*}\left(h_{i}\right)}{\left\{p^{n}\left(p^{i}-1\right)\right\}_{p}}$ is the unique element of finite order equal to $d_{i}$ in the group $\operatorname{Ext}_{B_{\Psi}^{k}}^{1,2 i}\left(k^{*}(P), k^{*}(P)\right), d_{i}=$ $\left\{p^{n}\left(p^{i}-1\right)\right\}_{p}, n \rightarrow \infty$.

The lemma is proved.

Note that the computation of $\operatorname{Ext}_{B_{\Psi}^{k}}^{* *}\left(k^{*}(P), k^{*}(P)\right)$ presents no difficulties, since the module $k^{*}(P)$ has a $B_{\Psi}^{k}$-free resolution which coincides with the complex for the polynomial algebra $Z\left[\gamma_{2}, \ldots, \gamma_{p}, \ldots\right]$, as long as the operator $x$ acts freely on $k^{*}(P), B_{\Psi}^{k}$.

We have

Theorem 8.1. The groups $\operatorname{Ext}_{A_{\Psi}^{k}}^{1,2 i}\left(k^{*}(P), k^{*}(P)\right)$ are cyclic groups of order $d_{i}=$ $\left\{p^{n}\left(p^{i}-1\right)\right\}_{p}$, where $n$ is large.

Proof. It is easy to see that the algebra $A_{\Psi}^{k} \otimes Q$ is precisely the algebra of operations in $k$-theory $k^{*} \otimes Q$. Hence, by virtue of $\S 2$, we have:

$$
\operatorname{Ext}_{A_{\Psi}^{k} \otimes Q}^{s, *}\left(k^{*}(P) \otimes Q, k^{*}(P) \otimes Q\right)=\operatorname{Ext}_{A_{\Psi}^{k}}^{s, *}(k(P), k(P) \otimes Q)=0
$$

for $s>0$. Hence the groups $\operatorname{Ext}_{A_{\Psi}^{k}}^{1,2 i}$ are all torsion. We consider the resolution

$$
\left(\ldots \stackrel{d}{\rightarrow} \sum_{i} A_{\Psi, i}^{k} \stackrel{d}{\rightarrow} A_{\Psi}^{k} \stackrel{\varepsilon}{\rightarrow} k^{*}(P)\right)=C,
$$

where $d\left(u_{i}\right)=\delta_{i}(u)$, and $u_{i}, u$ are free generators of $C_{1}$ and $C_{0}$.

We consider the nonacyclic complex

$$
\left(\ldots \stackrel{\bar{d}}{\rightarrow} \sum_{i} A_{\Psi, i}^{k} \stackrel{\bar{d}}{\rightarrow} A_{\Psi}^{k} \stackrel{\varepsilon}{\rightarrow} k^{*}(P)\right)=\bar{C}
$$


where $\bar{d}\left(v_{i}\right)=x^{i} \delta_{i}(v)$ and $v_{i}, v$ are free generators. We shall show that the complex $\bar{C}$ is such that in the group

$$
H_{1}\left(\operatorname{Hom}_{A_{\Psi}^{k}}^{*}\left(\bar{C}, k^{*}(P)\right), \bar{d}^{*}\right)
$$

the torsion part is exactly the same as in the group

$$
H_{1}\left(\operatorname{Hom}_{A_{\Psi}^{k}}^{*}\left(C, k^{*}(P)\right), d^{*}\right)=\operatorname{Ext}_{A_{\Psi}^{k}}^{1, *} .
$$

In fact, if $h_{j} \in \operatorname{Hom}_{A_{\Psi}^{k}}\left(\bar{C}, k^{*}(P)\right)$, where $h_{j}(v)=x^{j}(t)$, then

$$
\begin{aligned}
\left(\bar{d}^{*} h_{j}, v_{i}\right) & =h_{j}\left(x^{i} \delta_{i}(t)\right)=x^{i} \delta_{i} h_{j}(t) \\
& =x^{i} \delta_{i} x^{j}(t)=x^{i}\left(\delta_{i} x^{j}(t)\right) .
\end{aligned}
$$

Thus, if $d^{*} h_{j}=\sum \mu_{i}^{(j)} h_{j-i}^{(i)}$, where $h_{j-i}^{(i)}\left(u_{i}\right)=x^{j-i}(t)$, then

$$
\bar{d}^{*} h_{j}=\sum \mu_{i}^{(j)} h_{i}^{(j)}
$$

where $h_{j}^{(i)}\left(v_{i}\right)=x^{j}(t)$ and the numbers $\mu_{i}^{(j)}$ are the same. We note that the order of the group $\operatorname{Ext}_{A_{\Psi}^{k}}^{1,2 j}$ is precisely the greatest common divisor of the numbers $\mu_{i}^{(j)}$ as $i$ varies, and a generator is $d^{*}\left(h_{j}\right) /\left\{\mu_{i}^{(j)}\right\}_{i}$. Since the elements $\left(x^{i} \delta_{i}\right)$ give a system of relations in $k^{*}(P)$ over the ring $B_{\Psi}^{k} \subset A_{\Psi}^{k}$, the same integers $\mu_{i}^{(j)}$ give the torsion part of $\operatorname{Ext}_{B_{\Psi}^{k}}^{1,2 j}$, since the complex $\bar{C}$ over $A_{\Psi}^{k}$ is a segment of a $B_{\Psi}^{k}$-resolution of the module $k^{*}(P)$. By virtue of the lemma, we get the required result. The theorem is proved.

We now pass to the module $k^{*}(M U)$.

We have

Theorem 8.2. For any complex $X \in D$ there is a canonical isomorphism

$$
\operatorname{Hom}_{A_{\Psi}^{k}}^{*}\left(k^{*}(M U), k^{*}(X)\right)=U^{*}(X) .
$$

The proof of this assertion is essentially a straightforward consequence of the result of [22] concerning the fact that the Riemann-Roch theorem on the integrality of the number $\operatorname{ch} \xi T(X)[X]$ gives a complete set of congruence relations on Chern numbers in $\Omega_{U}$. More precisely: if $[X] \in \Omega_{U}$ indivisible element, then there exists $\xi \in K(X)$ such that $\operatorname{ch} \xi T(X)[X]=1$. By virtue of the properties of the Thom isomorphism in $K$-theory, this assertion is equivalent to the following: for any indivisible element $\alpha \in \pi_{*}(M U)$, there exists $\xi \in K^{0}(M U)$ such that $(\operatorname{ch} \xi, H \alpha)=1$, where $H: \pi_{*} \rightarrow H_{*}$ is the Hurewicz homomorphism. Let $\beta \in \operatorname{Hom}_{A_{\Psi}^{k}}^{*}\left(k^{*}(M U), k^{*}(P)\right)$; then the number $(\operatorname{ch} \xi, \beta)$ is also an integer by virtue of Bott periodicity. Both groups $\operatorname{Hom}_{A_{\Psi}^{k}}^{*}\left(k^{*}(M U), k^{*}(P)\right)$ and $\pi_{*}(M U)$ have no torsion. \{Note that $\operatorname{Hom}_{A_{\Psi}^{k}}\left(k^{*}(M U), k^{*}(P)\right) \subset k^{*}(P)$, for $k^{*}(M U)$ is cyclic on $u_{n}$. $\}$ Hence $\pi_{*}(M U) \subset \operatorname{Hom}_{A_{\Psi}^{k}}^{*}$. By virtue of what was said about the indivisibility of the numbers $(\operatorname{ch} \xi, H \alpha) \alpha \in \pi_{*}(M U)$, the group $\pi_{*}(M U)$ is indivisible in $\operatorname{Hom}_{A_{\Psi}^{k}}^{*}$. Since the ranks of these groups coincide, the groups coincide. Thus the assertion is proved for the point spectrum. 
Let $X \in D, X_{1}, X_{2} \in D$, with $X_{1}$ a skeleton of $X, X_{2}=X / X_{1}$; we have exact sequences:

$$
\begin{gathered}
0 \rightarrow U^{*}\left(X_{2}\right) \rightarrow U^{*}(X) \stackrel{i^{*}}{\rightarrow} U^{*}\left(X_{1}\right) \rightarrow 0, \\
0 \rightarrow k^{*}\left(X_{2}\right) \rightarrow k^{*}(X) \rightarrow k^{*}\left(X_{1}\right) \rightarrow 0 .
\end{gathered}
$$

We assume by induction that the theorem has been proved for $X_{1}$ and $X_{2}$ (we do induction on the rank of the group $H_{*}(X, Z)$ ). Then we have a commutative diagram of exact sequences:

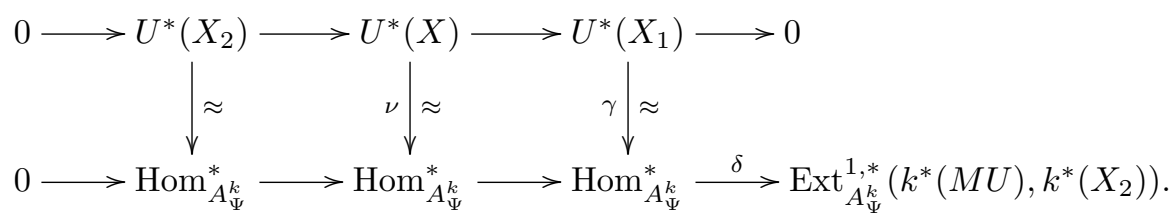

However, by virtue of the commutativity of the diagram we have that the homomorphism

$$
\operatorname{Hom}_{A_{\Psi}^{k}}^{*}\left(k^{*}(M U), k^{*}(X)\right) \rightarrow \operatorname{Hom}_{A_{\Psi}^{k}}^{*}\left(k^{*}(M U), k^{*}\left(X_{2}\right)\right)
$$

is an epimorphism, since $i^{*}$ is an epimorphism and $\gamma$ is an isomorphism. Hence the homomorphism $\delta$ is trivial, and hence by the 5-lemma the homomorphism $\nu$ is an isomorphism. The theorem is proved.

Remark 8.2. In what follows it will become clear that the groups $\operatorname{Ext}_{A_{\Psi}^{k}}^{i, *}\left(k^{*}(M U), k^{*}(P)\right)$ are nontrivial even for $i=1$, and the question of their computation is extraordinarily important (see $\S 9,11)$.

By analogy with the rings $A_{\Psi}^{k}$ and $B_{\Psi}^{k}$ it is possible to construct analogous rings $A_{\Psi}^{k O}$ and $B_{\Psi}^{k O}$. Let $k O^{*}$ be the theory defined by the spectrum $k O$ such that $\Omega^{8 n} k O_{n}=B O \times Z$ (see $\S 3$ ). The cohomology ring of a point $k O^{*}(P)=\Lambda_{O}$ is described as follows: generators $1 \in \Lambda_{O}^{0}, h \in \Lambda_{O}^{-1}, v \in \Lambda_{O}^{-4}, w \in \Lambda_{O}^{-8}$; relations $2 h=0, h^{3}=0, h v=0, v^{2}=4 w$.

We have the "complexification" operator

$$
c: k O^{*} \rightarrow k^{*}
$$

such that $c(h)=0, c(v)=2 x^{2}, c(w)=x^{4}$, where $x$ is the Bott periodicity operator.

In the theory $k O^{*}$ it is possible by analogy with the theory $k^{*}$ to introduce operations $\left(k^{n} \Psi^{k}\right)$ and their combinations $a=\left(a_{n}\right), a_{n}=\sum \lambda_{k}^{(n)}\left(k^{n} \Psi^{k}\right)$, where $a_{n *}^{(j)}$ does not depend on $n$. The ring of such operators is identical to the analogous ring for $k^{*}$-theory which lies in $B_{\Psi}^{k}$. The ring $B_{\Psi}^{k O}$ is composed, in a fashion identical to that for the ring $B_{\Psi}^{k}$, from such operators $a=\left(a_{n}\right)$ constructed from $\Psi^{k}$ and from the multiplication operators on $\Lambda_{O}=k O^{*}(P)$, keeping in mind the following commutativity relations: $\Psi^{k} h=k h \Psi^{k} ; \Psi^{k} v=k^{2} v \Psi^{k} ; \Psi^{k} w=k^{4} w \Psi^{k}$. We denote the resulting ring by $B_{\Psi}^{k O}$. Similarly, it is possible to construct a ring $A_{\Psi}^{k O}$ also, but we shall not consider this ring in what follows.

We consider the category $B \subset D \subset S$.

1) The spectral sequence $\left(E_{n}, d_{r}\right) \downarrow k O^{*}$ is trivial in $B$; in $B$ there is a subcategory $B^{\prime}$ such that:

2 ) the operation of the ring $B_{\Psi}^{k O}$ is well-defined in $B^{\prime}$. As is easy to see, the spheres $S^{n}$ (their spectra in $S$ ) lie in $B^{\prime}$ by definition, since $k O^{*}\left(S^{n}\right) \approx k O^{*}(P)$. 
If $f: S^{n+k} \rightarrow S^{n}$ is a mapping, then a necessary and sufficient condition for the complex $D^{n+k+1} \cup_{f} S^{n}$ to belong to $B^{\prime}$ is that $f^{*}=0, f^{*}: k O^{*}\left(S^{n}\right) \rightarrow k O^{*}\left(S^{n+k}\right)$.

In the category $B \supset B^{\prime}$ the operation of the $\operatorname{ring} \tilde{B}_{\Psi}^{k O}$ is well-defined, the latter being a priori an extension $B_{\Psi}^{k O} \rightarrow B_{\Psi}^{k}$, since in view of the presence of torsion in $A_{O}=k O^{*}(P)$ the operation is not defined by its own representation on $k O^{*}(P)$, in contrast to $k^{*}$-theory in the category $D$.

There is defined a homomorphism (epimorphism):

$$
\operatorname{Ext}_{B_{\Psi}^{k O}}^{1, *}\left(k O^{*}(P), k O^{*}(P)\right) \rightarrow \operatorname{Ext}_{\tilde{B}_{\Psi}^{k O}}^{1, *}\left(k O^{*}(P), k O^{*}(P)\right)
$$

and a Hopf invariant

$$
q_{1}: \operatorname{Ker} q_{0} \rightarrow \operatorname{Ext}_{\tilde{S}_{\Psi}^{k O}}^{1, *}\left(k O^{*}(P), k O^{*}(P)\right) .
$$

It is easy to see that the complexification $c: k O^{*} \rightarrow k^{*}$ is an algebraic functor (see Definition 9.1) from the category of $\tilde{B}_{\Psi}^{k O}$-modules to the category of $B_{\Psi}^{k O}$-modules.

It is also easy to show that

$$
\operatorname{Ext}_{B_{\Psi}^{k O}}^{1,4 k}\left(k O^{*}(P), k O^{*}(P)\right)=Z_{d_{k}}, \quad d_{k}=\left\{p^{n}\left(p^{2 k}-1\right)\right\}_{p}
$$

and

$$
\operatorname{Ext}_{B_{\Psi}^{k o}}^{0 s}\left(\Lambda_{O}, \Lambda_{O}\right)=Z_{2} \quad \text { for } \quad s=8 k+1,8 k+2
$$

We have a natural ring homomorphism $\tau: B_{\Psi}^{k O} \rightarrow B_{\Psi}^{k}$ generated by the homomorphism $c: k O^{*}(P) \rightarrow k^{*}(P)$, and consequently a homomorphism

$$
\begin{gathered}
\tilde{c}: \operatorname{Ext}_{B_{\Psi}^{k O}}^{1,4 k}\left(\Lambda_{O}, \Lambda_{O}\right) \rightarrow \operatorname{Ext}_{B_{\Psi}^{k}}^{1,4 k}(\Lambda, \Lambda), \\
\Lambda=k^{*}(P), \quad \Lambda_{O}=k O^{*}(P),
\end{gathered}
$$

whose image has, as is easy to see, index 1 for $k=2 l$ and index 2 for $k=2 l+1$, in consequence of the fact that the image of the homomorphism $c: k O^{t} \rightarrow k^{t}$ has index 1 for $t=8 l$ and 2 for $t=8 l+4$. Later, in $\S 9$, this homomorphism will be considered from another point of view.

There is defined an element $h \in \operatorname{Ext}_{B_{\Psi}^{k O}}^{0,1}\left(\Lambda_{O}, \Lambda_{O}\right)$ such that $2 h=0, h^{3}=0$, while multiplication by $h$

$$
\operatorname{Ext}_{B_{\Psi}^{k O}}^{0,8 k+1} \stackrel{h}{\rightarrow} \operatorname{Ext}_{B_{\Psi}^{k O}}^{0,8 k+2} \text { and } \operatorname{Ext}_{B_{\Psi}^{k O}}^{1, s} \rightarrow \operatorname{Ext}_{B_{\Psi}^{k O}}^{1, s+1}
$$

is a monomorphism for $s=8 k, 8 k+1$.

The images of the homomorphisms

$$
q_{0}: \pi_{*}\left(S^{n}\right) \rightarrow \operatorname{Ext}_{B_{\Psi}^{k O}}^{0, *}\left(\Lambda_{O}, \Lambda_{O}\right)
$$

and

$$
q_{1}: \pi_{*}\left(S^{n}\right) \rightarrow \operatorname{Ext}_{B_{\Psi}^{k O}}^{1, *}\left(\Lambda_{O}, \Lambda_{O}\right)
$$

are easy to study: namely, $q_{0}$ is an epimorphism (see [9]), and the image $\operatorname{Im} q_{1}$ is realized by the image of $q_{1} \circ J$, where $J: \pi_{*}(S O) \rightarrow \pi_{*}\left(S^{n}\right)$, and is nontrivial in dimensions $(1,4 k),(1,8 k+1),(1,8 k+2)$. 


\section{§ 9. Relations between different cohomology theories. Generalized HOPF INVARIANT. $U$-COBORDISM, $k$-THEORY, $Z_{p}$-COHOMOLOGY}

Let $X \in \vec{S}$ be a cohomology theory. Suppose given a subcategory $B \in \vec{S}$. We define the notion of the "Steenrod ring" $A_{B}^{X}$ of the theory $X^{*}$ in the subcategory $B$ : the ring $A_{B}^{X}$ is the set of transformations $\theta_{K}: X^{*}(K) \rightarrow X^{*}(K)$ which commute with the morphisms of the category $B$ (according to Serre). The ring $A_{B}^{X}$ contains the factor-ring $A^{X} / J(B)$, where $J(B)$ consists of all operations which vanish on the category $B$.

We now define "the generalized Hopf invariant:" let

$$
g: K_{1} \rightarrow K_{2}
$$

be a morphism in $B$ such that the object $C K_{1} \cup_{g} K_{2}\left(=0+{ }_{K_{1}} K_{2}\right.$ in the notation of $\S 1$, i.e., the sum with respect to the inclusions $K_{1} \stackrel{g}{\rightarrow}$ and $K_{1} \stackrel{g}{\rightarrow} K_{2}$ ) also lies in $B$.

We have an exact sequence

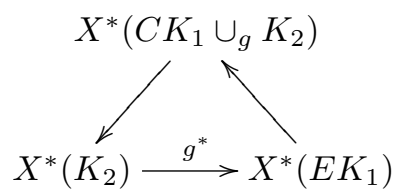

If the homomorphism $g^{*}=q_{0}(g): X^{*}\left(K_{2}\right) \rightarrow\left(E K_{1}\right)$ is trivial, then we have

$$
0 \rightarrow X^{*}\left(K_{1}\right) \rightarrow X^{*}\left(C K_{1} \cup_{g} K_{2}\right) \rightarrow X^{*}\left(K_{2}\right) \rightarrow 0,
$$

where $X^{*}\left(K_{i}\right), X^{*}\left(C K_{1} \cup_{g} K_{2}\right)$ are modules, and our short exact sequence determines a unique element

$$
q_{1}(g) \in \operatorname{Ext}_{A_{B}^{X}}^{1, *}\left(X^{*}\left(K_{1}\right), X^{*}\left(K_{2}\right)\right)
$$

We thus obtain a mapping

$$
q_{1}: \operatorname{Ker} q_{0}^{(B)} \rightarrow \operatorname{Ext}_{A_{B}^{X}}^{1, *}\left(X^{*}\left(K_{1}\right), X^{*}\left(K_{2}\right)\right),
$$

where $q_{0}: \operatorname{Hom}^{*}\left(K_{1}, K_{2}\right) \rightarrow \operatorname{Hom}_{A_{B}^{X}}^{*}\left(X^{*}\left(K_{2}\right), X^{*}\left(K_{1}\right)\right), K_{1}, K_{2} \in B$ and $g \in$ $\operatorname{Ker} q_{0}^{(B)}$, provided $C K_{1} \cup_{g} K_{2} \in B$. This map is "generalized Hopf invariant."

General problem: which elements of $\operatorname{Ext}_{A_{B}^{1}}^{1, *}\left(X^{*}\left(K_{2}\right), X^{*}\left(K_{1}\right)\right)$ ate realized geometrically as images $q_{1}\left(\operatorname{Ker} q_{0}^{(B)}\right)$ ?

If $\bar{A}_{B}^{X} \in A_{B}^{X}$ is an arbitrary subring, then there is defined the usual homomorphism:

$$
i: \operatorname{Ext}_{A_{B}^{X}}^{* *}\left(X^{*}\left(K_{2}\right), X^{*}\left(K_{1}\right)\right) \rightarrow \operatorname{Ext}_{A_{B}^{X}}^{* *}\left(X^{*}\left(K_{2}\right), X^{*}\left(K_{1}\right)\right)
$$

and we set $\bar{q}_{1}=i q_{1}$, where $\bar{q}_{1}$ is the "Hopf invariant" of the subring $\bar{A}_{B}^{X} \subset A_{B}^{X}$.

Examples.

1. If $B$ consists of a single object $K$, then $A_{B}^{X}=\operatorname{End} X^{*}(K)$ and there is no Hopf invariant.

2. If $B$ consists of objects $K_{1}, K_{2}, L=C K_{1} \cup_{g} K_{2}$ and morphisms $g: K_{1} \rightarrow K_{2}$, $\beta: L \rightarrow E K_{1}, \alpha: K_{2} \rightarrow L$, where $g^{*}: X^{*}\left(K_{2}\right) \rightarrow X^{*}\left(K_{1}\right)$ is the trivial homomorphism, then the ring $A_{B}^{X}$ consists of all endomorphisms of $X^{*}(L)$ which preserve the image $\beta^{*} X^{*}\left(K_{1}\right) \subset X^{*}(L)$. In this case, the Hopf invariant $q_{1}^{(B)}(g) \in$ $\operatorname{Ext}_{A_{B}^{X}}^{1}\left(X^{*}\left(K_{1}, X^{*}\left(K_{2}\right)\right)\right.$ is defined, and is equal to zero if and only if $X^{*}(L)=$ 
$X^{*}\left(K_{1}\right)+X^{*}\left(K_{2}\right)$ (as groups). Of course, examples 1 and 2 are uninteresting. We go on now to the examples which interest us.

3. Let $B=D$ (complexes with no torsion) and $X^{*}=H^{*}\left(, Z_{p}\right)$. In this case $A_{B}^{X}=A /(\beta A+A \beta)$, where $\beta$ is the Bokštern homomorphism and $A$ is the Steenrod algebra (over $Z_{p}$ ).

There is a canonical isomorphism

$$
\operatorname{Ext}_{A}^{1, t}\left(Z_{p}, Z_{p}\right)=\operatorname{Ext}_{A / \beta A+A \beta}^{1, t}\left(Z_{p}, Z_{p}\right)
$$

for $t>1$, where $Z_{p}=H^{*}\left(P, Z_{p}\right), P$ is a point, and the Hopf invariant $q_{1}(D)$ coincides with the Hopf invariant $q_{1}$ for $K_{1}=K_{2}=P$.

4. Let $B=D$ and $X^{*}=k^{*}$. In this case $A_{B}^{X} \supset A_{\Psi}^{k}$, and the latter ring contains the ring $A^{X} / J(B)$ but apparently does not coincide with it. The Hopf invariant in this theory will be discussed later; the $\operatorname{Ext}_{A^{k}}^{1, *}\left(k^{*}(P), k^{*}(P)\right)$ were computed in $\S 8$. In $\S 8$ we considered the subring $B_{\Psi}^{k} \subset A^{k}$ and

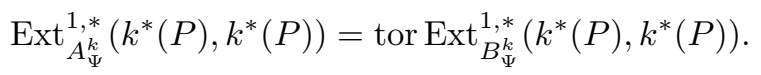

5. For the theory $X^{*}=U^{*}$ we shall also consider the category $B=D$ and the Hopf invariant for the whole ring $A^{U}$.

The groups $\operatorname{Ext}_{A^{U}}^{1, *}$ will be computed later (for $K_{2}=M S U$; see $\S 6$ ).

6. In $\S 2$ it was indicated that for complexes $K=E^{2} L$ the homomorphism $J: K^{0}(X) \rightarrow J(X)$ can be considered as a homorphism $J: K^{0}(X) \rightarrow P^{*}(X)$, where $P$ is the point spectrum or cohomotopy theory. A lower bound for the groups $\tilde{J}(X)$ can be computed in any cohomology theory $Y^{*}$, if we consider the composition

$$
q_{1}^{(Y)} \cdot J: K^{0}(X) \rightarrow P^{*}(X) \rightarrow \operatorname{Ext}_{A^{Y}}^{1, *}\left(Y^{*}(P), Y^{*}(X)\right),
$$

where $P^{*}(X)=\operatorname{Hom}^{*}(X, P)$, defined on elements such that $q_{0}^{(Y)} \cdot J=0$.

If $K=E L$, then in this case the computation can also be carried out by means of $\operatorname{Ext}_{A^{Y}}^{* *}\left(Y^{*}(P), Y^{*}(X)\right)$, but here the multiplicative structure in $\operatorname{Ext}_{A^{Y}}^{* *}$ y enters by virtue of Lemma 2.1 of $\S 2$.

We now consider two cohomology theories $X^{*}, Y^{*} \in \vec{S}$, a subcategory $B \subset S$ and a transformation $\alpha: X^{*} \rightarrow Y^{*}$ of the cohomology functors in the subcategory $B$. Let subrings $\bar{A}_{B}^{X} \subset A_{B}^{X}, \bar{A}_{B}^{Y} \subset A_{B}^{Y}$ be chosen.

Definition 9.1. We call the transformation $\alpha: X^{*} \rightarrow Y^{*}$ algebraic with respect to the subrings $\bar{A}_{B}^{X}, \bar{A}_{B}^{Y}$, if it induces a functor $\bar{\alpha}$ from the category of $\bar{A}_{B}^{X}$-modules to the category of $\bar{A}_{B}^{Y}$-modules. When $\bar{A}_{B}^{X}=A_{B}^{X}$, and $\bar{A}_{B}^{Y}=A_{B}^{Y}$ we call the transformation $\alpha$ algebraic.

\section{Examples.}

1. Let $X^{*}, Y^{*}$ be arbitrary cohomology theories. An arbitrary element $\alpha \in$ $Y^{*}(X)$ determines a transformation of theories

$$
\alpha: X^{*} \rightarrow Y^{*} .
$$

2. If the theory $X^{*}$ is such that $X^{i}(P)=0$ for $i>0$ and $X^{0}(P)=\pi$, then there arises an augmentation functor

$$
\nu: X^{*} \rightarrow H^{*}(Y, \pi)
$$

and hence for any group $G$ a functor

$$
\nu_{G}: X^{*} \rightarrow H^{*}(, \pi \otimes G) .
$$


For example, for $G=Z_{p}$ we have $\nu_{p}: X^{*} \rightarrow H^{*}\left(, \pi \otimes Z_{p}\right)$. In the cases of interest to us, $\pi=Z$ and $\pi \otimes Z_{p}=Z_{p}$.

3. The Riemann-Roch functor. Let $X^{*}=U^{*}$ and $Y^{*}=k^{*}$; we consider the Atiyah-Hirzebruch-Grothendieck element $\lambda_{1}^{(n)} \in K^{0}\left(M U_{n}\right)$. It defines a map

$$
\lambda_{-1}: U^{*} \rightarrow K^{*}
$$

and $\lambda: U^{*} \rightarrow k^{*}$, where $\lambda=\left(\lambda^{(n)}\right), \lambda^{(n)} \in k^{2 n}\left(M U_{n}\right)$, is the element (uniquely defined) such that $x^{n} \lambda^{(n)}=1 \in K^{0}\left(M U_{n}\right)$, where $x$ is the Bott operator.

For the theory $X^{*}=U^{*}$, the augmentation functors $\nu, \nu_{p}$ and the Riemann-Roch functor $\lambda$ preserve the ring structure of the theory.

Later it will be shown that these functors are algebraic in the category $D$.

Now let $\alpha: X^{*} \rightarrow Y^{*}$ be an algebraic transformation of theories in the category $B \subset S$ with respect to the subrings $\bar{A}_{B}^{X}, \bar{A}_{B}^{Y}$. What is the connection between the "Hopf invariants" $q_{1}^{(B)}$ in the theories $X^{*}$ and $Y^{*}$ ?

Since $\alpha: X^{*} \rightarrow Y^{*}$ leads to a functor in the category of modules, the trivial morphism $g_{X}^{*}: X^{*}\left(K_{2}\right) \rightarrow X^{*}\left(K_{1}\right)$ corresponds to the trivial morphism $g_{Y}^{*}: Y^{*}\left(K_{2}\right) \rightarrow$ $Y^{*}\left(K_{1}\right)$ for $K_{1}, K_{2}, g \in B$. Hence we have the inclusion $\operatorname{Ker} q_{0 X}^{(B)} \subset \operatorname{Ker} q_{0 Y}^{(B)}$, and the domain of definition of the Hopf invariant $\bar{q}_{1 X}^{(B)}$ is contained in the domain of definition of $\bar{q}_{1 Y}^{(B)}$.

Now let $\bar{\alpha}$ be a right exact functor in the category of modules. We consider a resolution $C_{x}$ of the module $M=X^{*}\left(K_{2}\right)$ and the following (commutative) diagram:

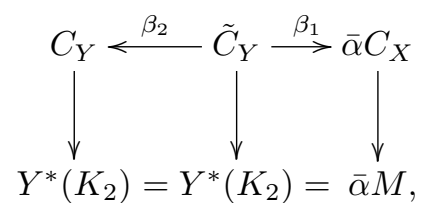

where $C_{Y}$ is an acyclic $\bar{A}_{B}^{Y}$-free resolution of the module $\bar{\alpha} M=Y^{*}\left(K_{2}\right), \tilde{C}_{Y}$ is a free complex such that $H^{0}\left(\tilde{C}_{Y}\right)=\bar{\alpha} M$. Let $N=X^{*}\left(K_{1}\right), \bar{\alpha} N=Y^{*}\left(K_{1}\right)$,

By definition we have: $H^{*}\left(\operatorname{Hom}_{\bar{A}_{B}^{Y}}^{*}\left(\bar{\alpha} C_{X}, \bar{\alpha} N\right)\right)=R^{*} G_{N}(M)$, where $R^{*}=\sum_{q} R^{q}$ and $G_{N}=\operatorname{Hom}_{\bar{A}_{B}^{Y}}^{*}(, \bar{\alpha} N) \circ \bar{\alpha}$ is the composite functor, $R^{q} G$ is the $q$-th right derived functor. There is defined a natural homomorphism

$$
r_{q}: \operatorname{Ext}_{\bar{A}}^{q, *}(M, N) \rightarrow R^{q} G_{N}(M), \quad r=\sum_{q} r_{q},
$$

and homomorphisms

$$
\begin{gathered}
\beta_{1}^{*}: R^{q} G_{N}(M) \rightarrow H^{q, *}\left(\operatorname{Hom}_{\bar{A}_{B}^{Y}}^{*}\left(\tilde{C}_{Y}, \bar{\alpha} N\right)\right) \\
\uparrow_{\beta_{2}^{*}} \\
\operatorname{Ext}_{\bar{A}_{B}^{Y}}^{q, *}(\bar{\alpha} M, \bar{\alpha} N),
\end{gathered}
$$

where $\operatorname{Ker} \beta_{2}^{*}=0$.

We have the composite map

$$
\tilde{\alpha}=\left(\beta_{2}^{*}\right)^{-1} \beta_{1}^{*} r_{1}: E_{1}(\bar{\alpha}) \rightarrow \operatorname{Ext}_{\bar{A}_{B}^{Y}}^{1}\left(Y^{*}\left(K_{2}\right), Y^{*}\left(K_{1}\right)\right),
$$


where

$$
\begin{gathered}
E_{1}(\bar{\alpha}) \subset \operatorname{Ext}_{\bar{A}_{B}^{X}}^{1}\left(X^{*}\left(K_{2}\right), X^{*}\left(K_{1}\right)\right), \\
E_{1}(\bar{\alpha})=r_{1}^{-1} \circ \beta_{1}^{*^{-1}} \beta_{2}^{*}\left(\operatorname{Ext}_{\bar{A}_{B}^{Y}}^{1}\left(Y^{*}\left(K_{2}\right), Y^{*}\left(K_{1}\right)\right) .\right.
\end{gathered}
$$

In the following cases the group $E_{1}(\bar{\alpha})$ coincides with the whole group Ext:

a) $\bar{\alpha}$ is an exact functor; here $H^{i}\left(\bar{\alpha} C_{X}\right)=0, i>0$, and one can assume that $\tilde{C}_{Y}=C_{Y}, \beta_{2}=1$.

b) If in addition $\bar{\alpha}$ is such that $\operatorname{Ext}_{\bar{A}_{B}^{Y}}^{i}(, \varepsilon N) \circ \varepsilon=0$ for $i>0$, then $\bar{\alpha} C_{X}=C_{Y}$ and an isomorphism is generated:

$$
\operatorname{Ext}_{\bar{A}}^{* *}(\bar{\alpha} M, \bar{\alpha} N)=R^{*} G_{N}(M) .
$$

In case (a) ( $\bar{\alpha}$ is an exact functor) there arises a spectral sequence $\left(E_{r}, d_{r}\right)$, where $E_{2}^{p, q}=\sum_{p, q} R^{p} G_{q, N}(M)$, which converges to $\operatorname{Ext}^{* *}(\bar{\alpha} M, \bar{\alpha} N)$, and $G_{q, N}(M)=$ $\operatorname{Ext}^{q, *}(, \varepsilon N) \circ \varepsilon$. From this spectral sequence it follows immediately that the homomorphism

$$
\beta_{1}^{*}: R^{1} G_{N}(M) \rightarrow \operatorname{Ext}^{1}(\bar{\alpha} M, \bar{\alpha} N)
$$

is a monomorphism.

The basic examples which we shall consider are the subcategory $D$ of torsion-free complexes, the theories $U^{*}, k^{*}, H^{*}\left(, Z_{p}\right)$, the Riemann-Roch functor $\lambda: U^{*} \rightarrow k^{*}$ and the augmentations $\nu_{p}: U^{*} \rightarrow H^{*}\left(, Z_{p}\right)$. We have

Lemma 9.1. a) The functors $\lambda: U^{*} \rightarrow k^{*}$ and $\nu_{p}: U^{*} \rightarrow H^{*}\left(, Z_{p}\right)$ are algebraic in the subcategory $D$;

b) The functors $\lambda$ and $\nu_{p}$ are exact in this category.

c) The functor $\lambda$ is such that $R^{q} G_{N}(M)=\operatorname{Ext}_{A^{U}}^{q}(M, N)$, where $M=U^{*}\left(K_{2}\right)$, $N=U^{*}\left(K_{1}\right), M, N \in D, C_{N}=\operatorname{Hom}_{A^{k}}(, \lambda N) \circ \lambda, \lambda N=k^{*}\left(K_{1}\right)$.

d) The functor $\nu_{p}$ is such that $R^{q} G_{N}(M)=\operatorname{Ext}_{A / \beta A+A \beta}^{q}\left(\nu_{p} M, \nu_{p} N\right)$.

Proof. The category of $A^{U}$-modules corresponding to the category $D$ is the category of $\Lambda$-free modules, where $\Lambda=U^{*}(P) \approx \Omega_{U}$. On the cohomology of a point $\Lambda$ the functor $\lambda$ is such that $\Lambda \stackrel{\lambda}{\rightarrow} Z[x]$ and $\lambda(y)=T(y) x^{i}$, where $y \in \Omega_{U}^{2 i}=U^{-2 i}(P)$ and $T$ is the Todd genus.

From the group point of view we have $\lambda M=M \otimes_{\Lambda} Z[x]$, where $M$ is $\Lambda$-free. There follows the exactness of the functor $\lambda$ and $R^{q} \lambda=0, q>0$. For $\nu_{p}$ we have $\nu_{p} U^{*}(P)=Z_{p}$, and in the category $D, \nu_{p} M=M \otimes_{\Lambda} Z_{p}$; since in the category $D$ all groups $U^{*}(K)$ and $H^{*}(K)$ are free abelian, the functor $\nu_{p}$ is exact in this category. This proves part (d). Part (c) follows immediately from the theorem in $\S 7$. Part (b) follows from the well-known fact that $H^{*}\left(M U, Z_{p}\right)$ is a free $(A / \beta A+A \beta)$-module. We shall now prove the fundamental part (a).

Consider first the functor $\lambda$. We recall that in $\S 5$ we constructed operations $\Psi_{U}^{k} \in A^{U} \otimes Q$. Let

$$
\Psi^{k}(\lambda \tilde{x})=\lambda \Psi_{U}^{k}(\tilde{x}), \quad \tilde{x} \in U^{*}(K)
$$

where $K \in D$ is a complex with no torsion. Since $\lambda$ is an epimorphism and $\lambda(y)=$ $T(y) x^{i}$ where $x$ is the Bott operator, the desired formula follows easily from the construction of the Adams operations $\Psi^{k}$ in $K$-theory and of the operations $\Psi_{U}^{k}$ in $\S 5$. The operations $\left(k^{n} \Psi^{k}\right)$ have the form $k^{n} \lambda \Psi_{U}^{k}$ and are "integral" for large 
$n$. Thus, the action of the operators $\left(k^{n} \Psi^{k}\right)$ and multiplication by $x$ in $k^{*}$-theory are calculated by $A^{U}$ and $\lambda$. This proves part (a) of the lemma for the functor $\lambda$.

Now let $\alpha=\nu_{p}: U^{*} \rightarrow H^{*}\left(, Z_{p}\right)$. In $\S 5$ we constructed a projector $\Phi \in$ $A^{U} \otimes_{Z} Q_{p}$ of the theory $U^{*}$ onto a smaller theory having the cohomology of a point $\Lambda_{p}=Q_{p}\left[x_{1}, \ldots, x_{i}, \ldots\right], \operatorname{dim} x_{i}=-2\left(p^{i}-1\right)$.

We set

$$
P^{k}\left(\nu_{p} \tilde{x}\right)=\nu_{p} \Phi S_{\omega} \Phi(\tilde{x}),
$$

where $\omega=(p-1, \ldots, p-1)$ ( $k$ times $)$ and the $P^{k}$ are the Steenrod powers. The correctness of this formula follows from the fact that all homomorphisms $\left(\Phi S_{\omega} \Phi\right)^{*}(y) \equiv 0 \bmod p$ if $\operatorname{dim} \omega=\operatorname{dim} y$, i.e., $\left(\Phi S_{\omega} \Phi\right)^{*}(y) \in \Omega_{U}^{0}=Z$. The lemma is proved.

Corollary 9.1. For any $K_{1}, K_{2} \in D$ the homomorphism

$$
\tilde{\alpha}=\tilde{\lambda}: \operatorname{Ext}_{A^{U}}^{1, *}\left(U^{*}\left(K_{2}\right), U^{*}\left(K_{1}\right)\right) \rightarrow \operatorname{Ext}_{A_{\Psi}^{k}}^{1, *}\left(k^{*}\left(K_{2}\right), k^{*}\left(K_{1}\right)\right)
$$

is a monomorphism.

Proof. As was established in Theorem 8.2, the homomorphism $r_{1}$ is an isomorphism; the homomorphism $\beta_{1}^{*}$ is a monomorphism, as was shown above, while $\beta_{2}^{*}=1$, since $R^{q} \lambda=0, q>0$. Hence, $\beta_{1}^{*} r_{1}=\tilde{\lambda}$ is a monomorphism.

Corollary 9.2. For any complex $K=E^{2} L$ the lower bound of the J-functor

$$
q_{1 k}^{(D)} \cdot J\left(K^{0}(X)\right) \in \operatorname{Ext}_{A_{\Psi}^{k}}^{1, *}\left(k^{*}(P), k^{*}(X)\right)
$$

coincides with the bound

$$
q_{1 U}^{(D)} \cdot J\left(K^{0}(X)\right) \in \operatorname{Ext}_{A^{U}}^{1, *}\left(U^{*}(P), U^{*}(X)\right)
$$

Corollary 9.2 follows from Corollary 9.1.

Corollary 9.3. The groups $\operatorname{Ext}_{A^{U}}^{1,2 i}\left(U^{*}(P), U^{*}(P)\right)$ are cyclic groups - subgroups of cyclic groups of order equal to the greatest common divisor of the integers $\left\{k^{n}\left(k^{i}-\right.\right.$ 1) $\}_{k}$ for all $k$, for large $n$.

Proof. Since the groups $\operatorname{Ext}_{A_{\Psi}^{k}}^{1,2 i}\left(k^{*}(P), k^{*}(P)\right)$ by virtue of the theorem are cyclic of the asserted orders, Corollary 9.3 follows from Corollary 9.1. We shall indicate a simple fact about the connection between the Hopf invariants in different cohomology theories $X^{*}, Y^{*}$ in the presence of an algebraic transformation $\alpha: X^{*} \rightarrow Y^{*}$ with respect to the rings $\bar{A}_{B}^{X}, \tilde{A}_{B}^{Y}$ in the subcategory $B \subset S$.

Lemma 9.2. We have the equality

$$
\bar{q}_{1 Y}^{(B)}=\tilde{\alpha} \cdot \bar{q}_{1 X}^{(B)}
$$

on $\operatorname{Ker} q_{0 X}^{(B)}$, the group $q_{1 X}^{(B)}\left(\operatorname{Ker} q_{0 X}^{(B)}\right)$ being contained in $E_{1}(\alpha)$, the domain of definition of the homomorphism $\tilde{\alpha}=\left(\beta_{1}^{*-1} \cdot \beta_{1}^{*} \cdot r_{1}\right)$.

The proof of this lemma follows immediately from the fact that by construction of the generalized Hopf invariants $\bar{q}_{1 X}^{(B)}$ and $\bar{q}_{1 Y}^{(B)}$ we can compute both quantities $\bar{q}_{1 X}^{(B)}(a)$ and $\bar{q}_{1 Y}^{(B)}(a)$ for any $a \in \operatorname{Ker} \bar{q}_{0 X}^{(B)} \subset \operatorname{Ker} \bar{q}_{0 X}^{(B)}$.

As is easy to see, the equality

$$
\beta_{2}^{*} q_{1 Y}^{(B)}(a)=\beta_{1}^{*} \cdot r_{1} \cdot \bar{q}_{1 X}^{(B)}(a)
$$


is true. This equation is equivalent to everything asserted by the lemma. The lemma is proved.

Corollary 9.4. a) If the element $\gamma \in \operatorname{Ext}_{\bar{A}_{B}^{X}}^{1, *}$ does not belong to $E_{1}(\alpha)$ for any algebraic $\alpha: X^{*} \rightarrow Y^{*}$, then the element $\gamma$ is not realized as the Hopf invariant of any element of $\operatorname{Hom}^{*}\left(K_{1}, K_{2}\right)$.

b) If $\gamma \in \operatorname{Ext}_{\bar{A}_{B}^{Y}}^{1, *}$ does not belong to the image of the homomorphism $\tilde{\alpha}\left(\operatorname{Ext}_{\bar{A}_{B}^{X}}\right)$ and $\operatorname{Ker} q_{0 X}^{(B)}=\operatorname{Ker} q_{0 Y}^{(B)}$, then the element $\gamma$ is not realized as the Hopf invariant of any element of $\operatorname{Hom}^{*}\left(K_{1}, K_{2}\right)$.

\section{$\S$ 10. Computation of $\operatorname{Ext}_{A U}^{1}\left(U^{*}(P), U^{*}(P)\right)$. Computation of Hopf} INVARIANTS IN CERTAIN THEORIES

In the preceding section the monomorphicity of the mapping

$$
\operatorname{Ext}_{A^{U}}^{1}\left(U^{*}(P), U^{*}(P)\right) \rightarrow \operatorname{Ext}_{A_{\Psi}^{k}}^{1}\left(k^{*}(P), k^{*}(P)\right)
$$

was established.

We shall now bound the order of the groups $\operatorname{Ext}_{A^{U}}^{1,2 i}$ from below. We consider the resolution

$$
\left(\ldots \stackrel{d}{\rightarrow} C_{1} \stackrel{d}{\rightarrow} C_{0} \stackrel{\varepsilon}{\rightarrow} U^{*}(P)=\Lambda\right)=C,
$$

where $C_{0}=A^{U}$ (generated by $u$ ) and $C_{1}=\sum_{\omega} A_{\omega}^{U}$ with generators $u_{\omega}, d u_{\omega}=S_{\omega}(u)$, $\operatorname{dim} \omega>0$. We consider the differential

$$
d^{*}: \Omega_{U}^{2 i} \rightarrow \sum_{\omega>0} \Omega_{U}^{2 i-2 \operatorname{dim} \omega}
$$

where

and

$$
d^{*}(x)=\sum_{\omega} \gamma_{\omega}^{*}, \quad x \in \Omega_{U}^{2 i}=\operatorname{Hom}_{A^{U}}^{2 i}\left(C_{0}, \Lambda\right)
$$

$$
\sigma_{\omega}^{*}(x) \in \operatorname{Hom}_{A^{U}}^{2 i}\left(C_{1}, \Lambda\right)=\sum_{\omega} \Omega_{U}^{2 i-2 \operatorname{dim} \omega},
$$

where $\sigma_{\omega}^{*}(x)\left[u_{\omega^{\prime}}\right]=0$ if $\omega \neq \omega^{\prime}$, and $\sigma_{\omega}^{*}(x)\left[u_{\omega}\right]=\sigma_{\omega}^{*}(x) \in \Lambda$. These facts follow from $\S 5$.

Now let $i$ be odd. We consider the element $x_{1}^{i}$, where $x_{1}=\left[C P^{1}\right] \in \Omega_{U}^{2}$. Since $\sigma_{1}^{*}\left(x_{1}\right)= \pm 2$, all $\sigma_{\omega}^{*}\left(x_{1}^{i}\right) \equiv 0 \bmod 2, \omega>0$, from the properties of the homomorphisms $\sigma_{\omega}^{*}$ described in $\S 5$. Hence the cokernel Coker $d^{*}$ always contains an element of order 2. Since the homomorphism $\operatorname{Ext}_{A^{U}}^{1,4 l+2} \rightarrow \operatorname{Ext}_{A_{\Psi}^{k}}^{1,4 l+2}$ is monomorphic and $\left\{k^{n}\left(k^{i}-1\right)\right\}_{k}=2, i \equiv 1(\bmod 2)$, we have

$$
\operatorname{Ext}_{A^{U}}^{1,4 l+2}\left(U^{*}(P), U^{*}(P)\right)=\operatorname{Ext}_{A_{\Psi}^{k}}^{1,4 l+2}\left(k^{*}(P), k^{*}(P)\right)=Z_{2}
$$

for $4 l+2=2 i, i \equiv 1(\bmod 2)$.

Thus, we have proved

Theorem 10.1. The groups $\operatorname{Ext}_{A^{U}}^{1,2 i}(\Lambda, \Lambda)$ are isomorphic to $Z_{2}$ for $i=2 l+1$.

We now study the case of even $i=2 l$. Let $y_{i} \in \Omega_{U}^{2 i}$ be an indivisible element such that some multiple $\lambda y_{i}, \lambda \neq 0$, represents an almost-parallelizable manifold $M^{2 i}$, whose tangent bundle $\tau$ is a multiple of the basic element $\kappa_{i}$ of the group $K^{0}\left(S^{2 i}\right), \tau=\mu_{i} \bar{\kappa}_{i}, \mu_{i}$ integral, where $\bar{\kappa}_{i}=f^{*} \bar{\kappa}_{i}, f: M^{2 i} \rightarrow S^{2 i}$ a projection of degree \pm 1 . From the requirement of the integrality of the Todd genus and the fact 
that $\left(\operatorname{ch} \bar{\kappa}_{1}, M^{2 i}\right)=1$, it follows easily that all $\sigma_{\omega}^{*} y_{i}$ for all $\omega$ are divisible by the denominator of the number $\left(B_{l} / a_{l} \cdot 2 l\right)$, where $a_{2 S+1}=1$ and $a_{2 S}=2, B_{l}$ is the Bernoulli number entering into the Todd genus, $i=2 l$ (see [14]). Hence Coker $d^{*}$ contains a group of order of equal of the denominator of the number $\left(B_{l} / a_{l} \cdot 2 l\right)$. Since this number is only half of the number $\left\{k^{n}\left(k^{i}-1\right)\right\}_{k}\left(a_{l}=1\right)$, the image $\tilde{\lambda} \operatorname{Ext}_{A^{U}}^{1,2 i} \subset \operatorname{Ext}_{A_{\Psi}^{k}}^{1,2 i}$ coincides with $\operatorname{Ext}_{A_{\Psi}^{k}}^{1,2 i}$ for $l=2 s$ and has index 2 in $\operatorname{Ext}_{A_{\Psi}^{k}}^{1,2 i}$ for $l=2 s+1$.

From this, for the case $a_{l}=2, l=2 s$, follows the

Theorem 10.2. The groups $\operatorname{Ext}_{A^{U}}^{1,8 k}(\Lambda, \Lambda)$ are isomorphic to the groups $\operatorname{Ext}_{A_{\Psi}^{k}}^{1,8 k}\left(k^{*}(P), k^{*}(P)\right)$.

In the case $a_{l}=1$ there arises an uncertainty: do the groups $\operatorname{Ext}_{A_{U}}^{1,8 k+4}$ coincide with the groups $\operatorname{Ext}_{\mathcal{A}_{\Psi}^{k}}^{1,8 k+4}$ or do they have index 2 in them?

Hence, we have the weaker

Theorem 10.3. The groups $\operatorname{Ext}_{A_{U}}^{1,8 k+4}(\Lambda, \Lambda)$ are cyclic groups whose order is equal to either the denominator of the number $B_{2 k+1} /(4 k+2)$ or the denominator of the number $B_{2 k+1} /(8 k+4)$.

Remark 10.1. In what follows it will be established that this order is in fact equal to the denominator of $B_{2 k+1} /(8 k+4)$ for $k \geq 1$ (however, for $k=0$ it is easy to see that $\left.\operatorname{Ext}_{A^{U}}^{1,4}(\Lambda, \Lambda)=Z_{12}\right)$. The basis element $u_{k}$ of the group $\operatorname{Ext}_{A^{U}}^{1,8 k+4}$ is such that

$$
d_{3}\left(u_{k}\right)=h^{3} \operatorname{Ext}_{A^{U}}^{1,8 k}(\Lambda, \Lambda), \quad h \in \operatorname{Ext}_{A^{U}}^{1,2}(\Lambda, \Lambda)=Z_{2} .
$$

We now study the question of the relations among different cohomology theories and the question of the existence of elements in the homotopy groups of spheres with given Hopf invariant

$$
\gamma \in \operatorname{Ext}_{\bar{A}_{D}^{X}}^{1, *}\left(X^{*}(P), X^{*}(P)\right)
$$

for the cases $X^{*}=U^{*}, k^{*}, k O^{*}, H^{*}\left(, Z_{p}\right)$, with the help of the functors $\alpha=\lambda$, $\alpha=c, \alpha=\nu_{p}$ relating these theories.

1. The first question which we consider here is the complexification

$$
c: k O^{*} \rightarrow k^{*}
$$

with respect to the rings $B_{\Psi}^{k O}$ and $B_{\Psi}^{k}$. The structure of the groups

$$
\operatorname{Ext}_{B_{\Psi}^{k O}}^{s, *}\left(k O^{*}(P), k O^{*}(P)\right)
$$

where $s=0,1$ is known to us, namely:

$$
\begin{array}{ll}
\text { a) } & \operatorname{Ext}_{B_{\Psi}^{k o}}^{0, t}\left(k O^{*}(P), k O^{*}(P)\right)=Z_{2}, \quad t=8 k+1,8 k+2, k \geq 0, \\
& \operatorname{Ext}_{B_{\Psi}^{k O}}^{0 t}\left(k O^{*}(P), k O^{*}(P)\right)=0, \quad t \neq 8 k+1,8 k+2 ; \\
\text { b) } \quad \operatorname{Ext}_{B_{\Psi}^{k}(, 4}^{1,4}\left(k O^{*}(P), k O^{*}(P)\right)=Z_{\left\{k^{n}\left(k^{q}-1\right)\right\}_{k}}+\ldots, \quad n \rightarrow \infty \\
& \operatorname{Ext}_{B_{\Psi}^{k e}}^{1, k+t}\left(k O^{*}(P), k O^{*}(P)\right)=Z_{2}+\ldots
\end{array}
$$

for $t=1,2$;

c) the homomorphism $q_{0}: \pi_{*}^{S}(P) \rightarrow \operatorname{Hom}_{B_{\Psi}^{k O}}^{*}\left(k O^{*}(P), k O(P)\right)$ is an epimorphism (result of Brown-Peterson-Anderson [9]); 
d) the homomorphism $q_{1} \cdot J: \widetilde{k O^{t}}(P) \rightarrow \operatorname{Ext}_{B_{\Psi}^{k O}}^{1, t}\left(k O^{*}(P), k O^{*}(P)\right)$ is an epimorphism. This last fact follows from the work of Adams [3] for the groups $\operatorname{Ext}_{B_{\Psi}^{k O}}^{1,4 k}\left(\Lambda_{O}, \Lambda_{O}\right) ;$ since

$$
\operatorname{Ext}_{B_{\Psi}^{k O}}^{1,8 k+t}=h^{t} \operatorname{Ext}_{B_{\Psi}^{k O}}^{1,8 k} \quad(t=1,2), \quad h \in \operatorname{Ext}_{B_{\Psi}^{k O}}^{0,1},
$$

the required fact follows for the groups $\operatorname{Ext}_{B_{\Psi}^{k O}}^{1,8 k+t}\left(\Lambda_{O}, \Lambda_{O}\right)$.

We now consider the complexification $c$, defining homomorphisms $\tilde{c}, \tilde{c}^{\prime}$ :

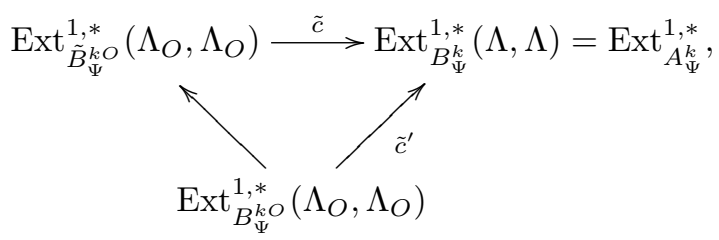

Since in the groups $k^{t}(P)$ the image of the homomorphism $c$ has index 2 for $t=$ $8 k+4$, index 1 for $t=8 k$ and is equal to zero for $t \neq 8 k, 8 k+4$, we can draw from this the conclusion that the image group $\operatorname{Im} \tilde{c} \subset \operatorname{Ext}_{A_{\Psi}^{k}}^{1, t}(\Lambda, \Lambda)$ has index 2 for $t=8 k+4$, index 1 for $t=8 k$ and is equal to zero for $t \neq 8 k, 8 k+4$, since $\operatorname{Im} \tilde{c}=\operatorname{Im} \tilde{c}^{\prime}$.

Consider the groups $\pi_{n+4 k-1}\left(S^{n}\right)$ and the Hopf invariants in $k O^{*}$ - and $k^{*}$ theories. These invariants are always defined since $\operatorname{Ext}_{\tilde{B}_{\Psi}^{k O}}^{0,4 k-1}=0$. We have thus the

Conclusion. The image of the Hopf invariant

$$
q_{1, k}: \pi_{n+4 k-1}\left(S^{n}\right) \rightarrow \operatorname{Ext}_{A_{\Psi}^{k}}^{1,4 k}\left(k^{*}(P), k^{*}(P)\right), \quad n \rightarrow \infty,
$$

has index 2 for $k=2 l+1$ and index 1 for $k=2 l$. Moreover, the image $q_{1, k}\left(\pi_{n+4 k-1}\left(S^{n}\right)\right)$ coincides with the image $q_{1, k} \cdot J \pi_{4 k-1}(S O)$.

2. We now consider the Riemann-Roch functor $\lambda: U^{*} \rightarrow k^{*}$ and the corresponding homomorphism $\tilde{\lambda}$ : $\operatorname{Ext}_{A^{U}}^{1, t}(\Lambda, \Lambda) \rightarrow \operatorname{Ext}_{A_{\Psi}^{k}}^{1, t}$. Since $\tilde{\lambda}$ is a monomorphism, we get from item 1 on complexification the following conclusion:

The Hopf invariant $q_{1 U}: \pi_{n+4 k-1}\left(S^{n}\right) \rightarrow \operatorname{Ext}_{A^{U}}^{1,4 k}$ is always defined, and its image $\operatorname{Im} q_{1 U}$ coincides with $q_{1 U}\left(J \pi_{2 k-1}(S O)\right)$; it coincides with $\operatorname{Ext}_{A^{U}}^{1,4 k}(\Lambda, \Lambda)$ for $k=1$, $k=2 l$ and has index 2 in the group $\operatorname{Ext}_{A^{U}}^{1,8 l+4}$ for $l \geq 1$.

Later we shall study $\operatorname{Ext}_{A^{U}}^{1,8 k+2}$ and $\operatorname{Ext}_{A^{U}}^{1,8 k+6}$.

3. We now consider the functor $\nu_{p}: U^{*} \stackrel{A}{\rightarrow} H^{*}\left(, Z_{p}\right)$ and the corresponding Hopf invariant

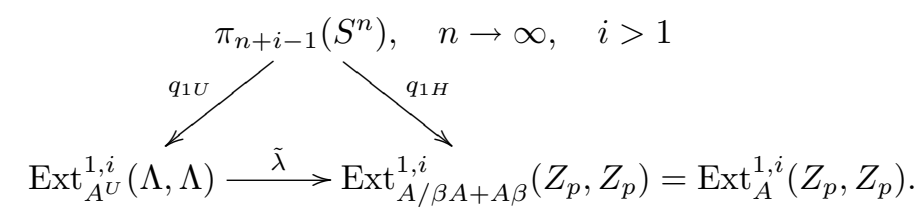

Since $\operatorname{Ext}_{A / \beta A+A \beta}^{1, i}\left(Z_{p}, Z_{p}\right)=\operatorname{Ext}_{A}\left(Z_{p}, Z_{p}\right)$ for $i>1$ this becomes the usual Hopf invariant. Since the homomorphism

$$
q_{1 U}: J \pi_{8 k-1}(S O) \rightarrow \operatorname{Ext}_{A^{U}}^{1,8 k}(\Lambda, \Lambda)
$$


is an epimorphism, the question of the existence of elements with ordinary Hopf invariant equal to 1 reduces to the calculation of this invariant on the group $J \pi_{4 k-1}(S O)$. For example, let $p=2$, and let $h_{i} \in \operatorname{Ext}_{A}^{1,2^{i}}\left(Z_{2}, Z_{2}\right)$ be basis elements. Since $h_{1}, h_{2}, h_{3}$ are cycles for all Adams differentials and represent elements in the groups $J \pi_{*}(S O)$, it follows that, in view of the fact that $\operatorname{Im} J$ is closed under composition, $h_{i} \cdot h_{2} \in \operatorname{Ext}_{A}^{2, *}\left(Z_{2}, Z_{2}\right)$ must represent an element of $q_{2} J \pi_{*}(S O)$ if $h_{i}$ represents an element of $q_{1} J \pi_{*}(S O)$. Moreover, since $q_{2} J \pi_{4 k-2}(S O)=0$, we have $h_{2} h_{i}=0$ if $h_{i} \in q_{1} J \pi_{*}(S O)$, since $h_{2} \in q_{1} J \pi_{*}(S O)$.

However, $h_{i} \cdot h_{1} \neq 0$ for $i \geq 4$. We have thus the

Conclusion. For $i \geq 4$ the elements $h_{i} \in \operatorname{Ext}_{A}^{1,2^{i}}\left(Z_{2}, Z_{2}\right)$ do not belong to the image of the homomorphism

$$
\nu_{2}: \operatorname{Ext}_{A^{U}}^{1,2^{i}}(\Lambda, \Lambda) \rightarrow \operatorname{Ext}_{A}^{1,2^{i}}\left(Z_{2}, Z_{2}\right)
$$

The case $p>2$ is considered analogously.

In fact, we have the purely algebraic

Theorem 10.4. The image of the homomorphism

$$
\nu_{p}: \operatorname{Ext}_{A^{U}}^{1,2 p^{i}(p-1)} \rightarrow \operatorname{Ext}_{A / A \beta+\beta A}^{1,2 p^{i}(p-1)}\left(Z_{p}, Z_{p}\right)
$$

is nontrivial only for $i=0,1,2(p=2)$ and for $i=0(p>2)$.

4. We now consider the homomorphism

$$
\delta: \operatorname{Ext}_{A^{U}}^{* *}\left(U^{*}(P), U^{*}(P)\right) \rightarrow \operatorname{Ext}_{A^{U}}^{* *}\left(U^{*}(M S U), U^{*}(P)\right) .
$$

We assume that $K \in \operatorname{Ext}_{A^{U}}^{0,4}\left(U^{*}(M S U), \Lambda\right), y \in \operatorname{Ext}_{A^{U}}^{0,8}\left(U^{*}(M S U), \Lambda\right)$, and $h \in$ $\operatorname{Ext}_{A^{U}}^{1,2}\left(U^{*}(M S U), \Lambda\right)$ are elements such that $d_{3}(K)=h^{3}$, and $y \in \Omega_{U}^{8}$ is represented by an almost-parallelizable manifold. We have

Lemma 10.1. All elements of the form $h^{n+1} \cdot K^{\varepsilon} \cdot y^{m}, n \geq 0, m \geq 0, \varepsilon=0,1$, belong to $\operatorname{Im} \delta$.

Proof. Since $h \in \operatorname{Im} \delta$, it suffices to show that $K^{\varepsilon} \cdot y^{m} \cdot h$ belongs to $\operatorname{Im} \delta$. For this it suffices to establish that all homomorphisms $\sigma_{\omega}^{*}\left(x_{1} \cdot K^{\varepsilon} \cdot y^{m}\right)$ are divisible by 2 . It is easy to verify that $\sigma_{\omega}^{*}\left(x_{1}\right), \sigma_{\omega}^{*}(K)$ and $\sigma_{\omega}^{*}\left(y^{m}\right)$ are divisible by 2 . The general result follows from the Leibnitz formula

$$
\sigma_{\omega}^{*}(a b)=\sum_{\omega=\left(\omega_{1}, \omega_{2}\right)} \sigma_{\omega_{1}}^{*}(a) \sigma_{\omega_{2}}^{*}(b)
$$

The lemma is proved.

As was shown in $\S 6$, in the Adams spectral sequence for $U^{*}(M S U)$ we have:

a) $d_{3}\left(h K y^{n}\right)=h^{4} y^{m} \neq 0$,

b) $d_{i}\left(h y^{m}\right)=0, i \geq 2$.

Moreover, Brown-Peterson-Anderson showed in [9] that elements of the form $h y^{m} \in$ $\Omega_{S U}^{8 m+1}$ belong to the image of the homomorphism $\pi_{*}\left(S^{n}\right) \rightarrow \pi_{*}\left(M S U_{n}\right)$ by a direct construction of the elements.

We have thus the

Theorem 10.5. a) The groups $\operatorname{Ext}_{A^{U}}^{1,8 k+2}(\Lambda, \Lambda)=Z_{2}$ are cycles for all Adams differentials and belong to the image of the Hopf invariant

$$
q_{1 U}: \pi_{n+8 k+1}\left(S^{n}\right) \rightarrow \operatorname{Ext}_{A^{U}}^{1,8 k+2}(\Lambda, \Lambda), \quad n \rightarrow \infty .
$$


b) The groups $\operatorname{Ext}_{A^{U}}^{1,8 k+6}(\Lambda, \Lambda)=Z_{2}$ are not cycles for the differential $d_{3}$.

Remark 10.2. Since $\operatorname{Ext}_{A^{U}}^{1,4 i+2}=\operatorname{Ext}_{A_{\Psi}^{k}}^{1,4 i+2}$, the analogous facts hold also for $k$ theory, although basis elements here are not related to the $J$-functor, in contrast to $\operatorname{Ext}_{A_{\Psi}^{k}}^{1,4 k}$ (here, the elements go into $h y^{m}$ under the homomorphism $\Omega_{e} \rightarrow \Omega_{S U}$ ).

We summarize the results of this section:

1) The groups $\operatorname{Ext}_{A^{U}}^{1, *}(\Lambda, \Lambda)$ were considered and also the associated homomorphisms

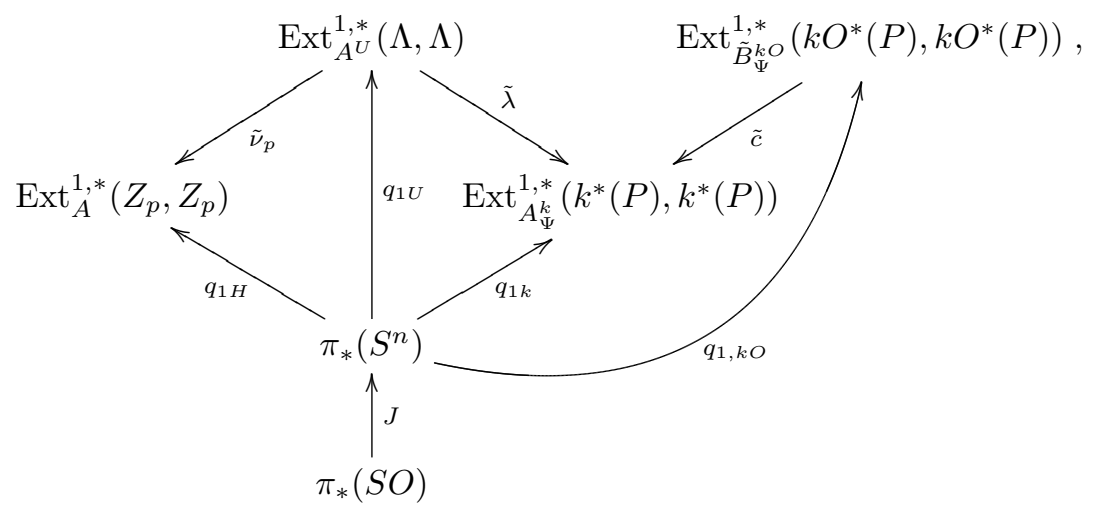

where $q_{1 H}$ is the classical Hopf invariant, $J$ is the Whitehead homomorphism, $\lambda$ is the "Riemann-Roch" functor, $c$ is complexification, and $\nu_{p}$ is the augmentation of $U^{*}$-theory into $Z_{p}$-cohomology theory.

2) The homomorphism $\operatorname{Ext}_{A^{U}}^{1}(\Lambda, \Lambda) \rightarrow \operatorname{Ext}_{A^{U}}^{1}\left(U^{*}(M S U), \Lambda\right)$ was studied.

3) It was established which elements of all these groups $\operatorname{Ext}^{1}$ are realized as images of the Hopf invariant $q_{1}$. In particular, for the groups $\operatorname{Ext}_{A^{U}}^{1,2 t}(\Lambda, \Lambda)$ this image $\operatorname{Im} q_{1 U}$ is trivial for $t=4 k+3 ; q_{1 U}$ is an epimorphism for $t=4 k-1,4 k$; for $t=4 k+2(k \geq 1)$ and $t=4 k+3(k \geq 0)$ the Adams differential

$$
d_{3}: \operatorname{Ext}_{A^{U}}^{1,2 t}(\Lambda, \Lambda) \rightarrow \operatorname{Ext}_{A^{U}}^{4,2 t+2}(\Lambda, \Lambda)
$$

is nontrivial; it can be shown that $d_{3}\left(E_{3}^{1,2 t}\right)=h^{3} E_{3}^{1,2 t-4}$ for $t=4 k+2(k \geq 1)$ and $t=4 k+3(k \geq 0)(\operatorname{see} \oint 11)$.

4) The nonexistence of elements with classical Hopf invariant 1 is a consequence of the fact that $\tilde{\nu}_{2}\left(\operatorname{Ext}_{A^{U}}^{1,2^{i}}\right)=0$ for $i \geq 4$. Analogously for $p>2$ (see $\left.\S 12\right)$.

5 . For $t \neq 8 k+4$, the fact of the following group isomorphism was established:

$$
\operatorname{Ext}_{A^{U}}^{1, t}(\Lambda, \Lambda) \stackrel{\tilde{\lambda}}{=} \operatorname{Ext}_{A_{\Psi}^{k}}^{1, t}\left(k^{*}(P), k^{*}(P)\right) ;
$$

for $t=4$ this fact is false. For $t=8 k+4, k \geq 1$, it is true and will be proved later (see $\S 11)$.

\section{$\S$ 11. Cobordism theory in the CATEgory $S \otimes_{Z} Q_{p}$}

Earlier, in $\S 5$, it was proved that in the algebra $A^{U} \otimes_{Z} Q_{p}$ there exists a projector $\Phi \in A^{U} \otimes_{Z} Q_{p}$ such that $\Phi(x, y)=\Phi(x) \Phi(y)$ and $\operatorname{Im} \Phi^{*} \subset \Lambda$ is the ring of polynomials in generators $y_{1}, \ldots, y_{i}, \ldots, \operatorname{dim} y_{i}=2 p^{i}-2$, where the $y_{i}$ are polynomial generators of the ring $\Lambda=\Omega_{U} \otimes_{Z} Q_{p}$ such that the numbers $\sigma_{\varepsilon}^{*}(y) \in Q_{p}$ are divisible by $p$ and $\sigma_{k}^{*}\left(y_{i}\right)=p, k=p^{i}-1$. Moreover, a complete system of 
orthogonal projectors $\Phi^{(i)}$ was constructed, $\sum \Phi^{(i)}=1, \Phi^{(i)} \cdot \Phi^{(j)}=0, i \neq j$, where the $\Phi^{(i)}\left(U^{*} \otimes_{Z} Q_{p}\right)$ are isomorphic theories up to shift of dimensions. Hence, in the category $S \otimes_{Z} Q_{p}$ the spectrum $M U$ is equal to the sum $M U \approx \sum_{\omega} E^{2 d(\omega)} M_{p}$, where $\omega$ is not $p$-adic $\left\{\right.$ i.e., $\omega=\left(i_{1}, \ldots, i_{k}\right)$, all $i_{q} \neq p^{r}-1$ for any $r$, and $\left.d(\omega)=\sum_{q=1}^{k} i_{q}\right\}$. If $A_{p}^{U}$ is the Steenrod ring of the spectrum $M_{p}$, where $A_{p}^{U}=\Phi \cdot A^{U} \cdot \Phi$, then we have:

1) $A^{U} \otimes_{Z} Q_{p}=G L\left(A_{p}^{U}\right)$ is the appropriately graded ring of infinite matrices of the form $\left(a_{\omega_{i} \omega_{j}}\right), a_{\omega_{i} \omega_{j}} \in A_{p}^{U}, \omega_{i}$ not $p$-adic and $\operatorname{dim}\left(a_{\omega_{i} \omega_{j}}\right)=2 d\left(\omega_{j}\right)-2 d\left(\omega_{j}\right)+$ $\operatorname{dim} a_{\omega_{i} \omega_{j}}$ \{i.e., the right-hand side is a constant for the whole matrix and defines the degree of the matrix $\}$.

2) $\operatorname{Ext}_{A^{U}}^{s, t}\left(U^{*}(K), U^{*}(L)\right) \otimes_{Z} Q_{p}=\operatorname{Ext}_{A_{p}^{U}}^{s, t}\left(U_{p}^{*}(K), U_{p}^{*}(L)\right)$, where $U_{p}^{*}=\Phi\left(U^{*} \otimes_{Z}\right.$ $\left.Q_{p}\right)$ is the theory defined by the spectrum $M_{p}$.

3) The Adams spectral sequences $\left(E_{r} \otimes Q_{p}, d_{r} \otimes Q_{p}\right)$ in $U$-theory and $\left(\tilde{E}_{r}, \tilde{d}_{r}\right)$ in $U_{p}$-theory coincide. These facts follow from $\S \S 1-3$.

We note that the polynomial generators of the ring $\Lambda_{p}=U_{p}^{*}(P)=\Phi^{*} U^{*}(P)$ can be chosen to be polynomials with rational coefficients in the elements $x_{i}=$ $\left[C P^{p^{i}-1}\right] \in \Omega_{U}$, where the polynomial generator can be identified with $\left[C P^{p-1}\right]=$ $x_{1}=y_{1}$ in the first nontrivial dimension, equal to $p-1$.

We consider the ring $\Lambda_{p, i} \subset \Lambda$, generated by the first $i$ polynomial generators $y_{1}, \ldots, y_{i} \in \Lambda_{p}$. This ring $\Lambda_{p, i}$ does not depend on the choice of generators.

The following fact is clear: the subring $\Lambda_{p, i} \subset \Lambda_{p}$ is invariant with respect to the action of all operations $\Phi \cdot S_{2} \cdot \Phi$ on the ring $\Lambda_{p}$. The proof follows from the fact that the subring $\Lambda_{(j)} \subset \Lambda=\Omega_{U}$, generated by all generators of dimension $\leq 2 j$, is invariant with respect to $S_{\omega}$ and with respect to $\Phi$, while $\Phi\left(\Lambda_{p^{i}-1}\right)=\Lambda_{p, i}$.

We consider the projection operator $\Phi_{i} \in A_{p}^{U} \otimes_{Q_{p}} Q$ such that $\Phi_{i}^{*}: \Lambda_{p} \rightarrow$ $\Lambda_{p, i}, \Phi_{i} \mid \Lambda_{p, i}=1$ and $\Phi_{i}\left(y_{j}\right)=0$ for $j>i$. The ring $\Phi_{i} A_{p}^{U} \Phi_{i}$ will be denoted by $A_{p, i}$. It is generated by the operators of multiplication by elements of $\Lambda_{p, i} \subset A_{p, i}$ and by operators of the form $\Phi_{i} \cdot \Phi \cdot S_{\omega} \cdot \Phi \cdot \Phi_{i}$, where it is sufficient to take only partitions $\omega=\left(k_{1}, \ldots, k_{s}\right), k_{j}=p^{q i}-1$, while $q_{j} \leq i$.

We have the following general fact.

The ring $A_{p}^{U}$ is generated by operators of the form $\Phi \cdot S_{\omega} \cdot \Phi$ for $\omega\left(k_{1}, \ldots, k_{s}\right)$, $k_{j}=p^{q_{j}}-1$.

This fact follows easily from properties of the projector $\Phi$ and the structure of the spectrum $M_{p}$.

However, if $\omega=\left(p^{q_{1}}-1, \ldots, p^{q_{s}}-1\right)$ and at least one $q_{j}>i$, then clearly $\sigma_{\omega}\left(\Lambda_{p, i}\right)=0$. Hence in the ring $\Phi_{i} A_{p}^{U} \Phi_{i}$ it suffices to consider only $\Phi_{i} \cdot \Phi \cdot S_{\omega} \cdot \Phi \cdot \Phi_{i}$ for $\omega=\left(p^{p_{1}}-1, \ldots, p^{q_{s}}-1\right)$, where all $q_{j} \leq i$.

Additive bases for the rings $A_{p}^{U}$ and $A_{p, i}$ :

a) $A_{p}^{U}=\left(\Lambda_{p} \cdot S_{\omega}\right)^{\wedge}$, where $\omega$ is $p$-adic and $\wedge$ denotes completion (by formal series).

b) $A_{p, i}=\left(\Lambda_{p, i} \cdot S_{\omega}\right)^{\wedge}, \omega=\left(p^{j 1}-1, \ldots, p^{j s}-1\right), j_{k} \leq i$.

We consider the operations $e_{i, k}=S_{\left(p^{i}-1, \ldots, p^{i}-1\right)}$ ( $k$ times), regarded as elements of the ring $A_{p, i}$, i.e., $e_{i, k}=\Phi_{i} \Phi S_{\omega} \Phi \Phi_{i}$. Clearly, we have:

1) $\Delta\left(e_{i, k}\right)=\sum_{k=l+s} e_{i s} \otimes e_{i, l}$ (the projectors $\Phi_{i}$ and $\Phi$ preserve the diagonal);

2) $e_{i, k}^{*}\left(\Lambda_{p, i-1}\right)=0$; 
3) $e_{i, 1}^{*}\left(y_{i}\right)=p$, where $y_{i} \in \Lambda_{p, i} \subset \Lambda_{p}$ is the polynomial generator of dimension $p^{i}-1$.

We denote by $\mathcal{E}_{i} \subset A_{p, i}$ the subring generated by the elements $\left(e_{i, k}\right), k \leq 1$.

We denote by $D_{i}$ the subring of $A_{p, i}$ spanned by $\mathcal{E}_{i}$ and the operator of multiplication by the generator $y_{i}$, i.e., $D_{i}=Q_{p}\left[y_{i}\right] \mathcal{E}_{i}$.

We have the following

Lemma 11.1. a) The subring $\mathcal{E}_{1}$ commutes with all operators of multiplication $\Lambda_{p, i-1} \subset \Lambda_{p}$ and all operators $\Phi_{i} \Phi S_{\omega} \Phi \Phi_{i}$ for all $\omega=\left(p^{j_{1}}-1, \ldots, p^{j_{s}}-1\right)$, where $j_{k} \leq i-1$.

b) In the ring $\mathcal{E}_{i}$ we have the relations

$$
\begin{gathered}
e_{i, k} \cdot e_{i, s}=\left(\begin{array}{c}
k+s \\
s
\end{array}\right) \cdot e_{i, k+s}, \\
e_{i, k} \cdot y_{i}^{q}=\sum_{s+m=i} e_{i, m}^{*}\left(y^{q}\right) \cdot e_{i, s},
\end{gathered}
$$

where $e_{i, m}^{*}\left(y^{q}\right)=\left(\begin{array}{c}q \\ m\end{array}\right) p^{m} y^{q-m}$.

c) The ring $A_{p, i-1}$ is obtained from the ring $A_{p, i}$ by discarding the polynomial generator $y_{i}$ and then factoring the remaining subring $B_{p, i} \subset A_{p, i}$ by the ideal spanned by the central subalgebra $\mathcal{E}_{i}$ of $B_{p, i}$, where

$$
B_{p, i}=\left\{\Lambda_{p, i-1} \cdot\left(\Phi_{i} \Phi S_{\omega} \Phi \Phi_{i}\right)\right\}^{\wedge}
$$

for all $p$-adic $\omega$.

The proof of all parts of Lemma 11.1 follows easily from what has preceded.

Thus, the ring $A_{p, i}$ is obtained from the ring $A_{p, i-1}$ in the following way (in two steps):

Step 1. Without altering the "ring of scalars" $\Lambda_{p, i-1}$, we make a central extension of $\mathcal{E}_{i+1}$ by $A_{p, i}$ :

$$
0 \rightarrow \mathcal{E}_{i} \rightarrow B_{p, i} \rightarrow A_{p, i-1} \rightarrow 0
$$

with $\mathcal{E}_{i}$ acting trivially on $\Lambda_{p, i-1}$.

Step 2. We adjoin to the ring of scalars $\Lambda_{p, i-1}$ a polynomial generator $y_{i}$ of dimension $p^{i}-1$, setting $e_{i, k}^{*}\left(y_{i}\right)=\left(\begin{array}{l}q \\ k\end{array}\right) p^{k} y^{q-k}$ with all the consequences derived from this.

The ring $Q_{p}\left[y_{i}\right] \cdot B_{p, i}$ coincides with $A_{p, i}$, while the commutation rules for $y_{i}$ and $\Phi_{i} S_{\omega} \Phi_{i}$ are derived from part (b) of Lemma 11.1.

In particular, the action of the operators $\Phi_{i} S_{\omega} \Phi_{i}$ for $\omega=\left(p^{j_{1}}-1, \ldots, p^{j_{k}}-1\right)$ and for $j_{k}<i$ can also turn out to be nontrivial.

We shall denote $\Phi S_{\omega} \Phi$ by $P^{k}$ when $\omega=(p-1, \ldots, p-1)$ ( $k$ times $)$.

We denote $\Phi_{i} P^{k} \Phi_{i}$ by $P^{k}$. For $p=2$ we set $P^{k}=\mathrm{Sq}^{k}$.

As in the ordinary Steenrod algebra $\bmod p$, we have here the following fact: the operations $P^{k}$ together with $\Lambda_{p}$ generate the entire $\operatorname{ring} A_{p}^{U}$ (it suffices to take $P^{p^{s}}$ ). This follows easily from the fact that for the ring $A_{p}^{U} \otimes Z_{p}$ it is easily derived from the properties of the ordinary Steenrod algebra. Hence, it suffices to determine only the action of the operators $P^{k}$ on the generators $y_{i}$ (and even only of the $P^{p^{s}}$ ).

We now consider the ring $D_{i}$, operating on the module $Q_{p}\left[y_{i}\right]$, and the groups $\operatorname{Ext}_{D_{i}}^{s, t}\left(Q_{p}\left[y_{i}\right], Q_{p}\left[y_{i}\right]\right)$. We set

$$
\Gamma^{s,-t}=\operatorname{Ext}_{D_{i}}^{s, t}\left(Q_{p}\left[y_{i}\right], Q_{p}\left[y_{i}\right]\right) .
$$


We consider the groups $\Gamma^{s, t} \otimes \Lambda_{p, i-1}$. We have

Lemma 11.2. a) There is a well-defined graded action of the ring $A_{p, i-1}$ on $\sum \Gamma^{s, t} \otimes \Lambda_{p, i-1}$ such that:

1) $\lambda(x \otimes \mu)=x \otimes \lambda \mu, \lambda, \mu \in \Lambda_{p, i-1} \subset A_{p, i-1}$;

2) if $e_{i, \omega}=\Phi_{i} S_{\omega} \Phi_{i}$,

$$
e_{i, \omega} \in A_{p, i-1}, \quad \omega=\left(p^{i}-1, \ldots, p^{j_{s}}-1\right), \quad j_{j}<i,
$$

then

$$
e_{i, \omega}^{*}(x \otimes \mu)=\sum_{\omega=\left(\omega_{1}, \omega_{2}\right)} e_{i, \omega}^{*}(x) \otimes e_{i, \omega_{2}}^{*}(\mu),
$$

where $e_{i, \omega_{2}}^{*}\left(\Lambda_{p, i-1}\right)$ is the ordinary action and $e_{i, \omega_{2}}^{*}(x) \in \Gamma^{s} \otimes \Lambda_{p, i-1}$ for $x \in \Gamma^{s}$, $\mu \in \Lambda_{p, i-1}$

b) we have the equality

$$
\begin{aligned}
\operatorname{Hom}_{A_{p, i-1}}^{*}\left(A_{p, i-1}, \Gamma^{s, t}\right. & \left.\otimes \Lambda_{p, i-1}\right) \\
& =\operatorname{Hom}_{A_{p, i-1}}\left(A_{p, i-1}, \Lambda_{p, i-1}\right) \otimes \operatorname{Ext}_{D_{i}}^{s_{1}-t}\left(Q_{p}\left[y_{i}\right], Q_{p}\left[y_{i}\right]\right) .
\end{aligned}
$$

Proof. Part (b) is obvious. To construct the action of $A_{p, i-1}$ on $\Gamma^{s} \otimes \Lambda_{p, i-1}$ we note that the ring $B_{i}$ acts on $\Lambda_{p, i}=Q_{p}[y] \otimes \Lambda_{p, i-1}$ naturally, while the action is trivial on $\Lambda_{p, i-1}$. From this follows the natural action of the factor-ring $A_{p, i-1}$ on the groups

$$
\operatorname{Ext}_{D_{i}}\left(\Lambda_{p, i}, Q_{p}\left[y_{i}\right]\right)=\operatorname{Ext}_{D_{i}}\left(Q_{p}[y], Q_{p}[y]\right) \otimes \Lambda_{p, i-1},
$$

where $D_{i}=Q_{p}[y] \cdot \mathcal{E}_{1}$. It is now easy to derive part (a).

We note that the ring $B_{i}$ is a free right module over $\mathcal{E}_{i}$.

We have the following

Theorem 11.1. There exists a spectral sequence $\left(E_{2}, d_{r}\right)$, where:

a) $E_{\infty}$ is associated with $\operatorname{Ext}_{A_{p, i}}\left(\Lambda_{p, i}, \Lambda_{p, i}\right)$;

b) $E_{2}^{p, q}$ coincides with $\operatorname{Ext}_{A_{p, i-1}}^{p}\left(\Lambda_{p, i-1}, \Gamma^{q} \otimes \Lambda_{p, i-1}\right)$, where $\Gamma^{q} \otimes \Lambda_{p, i-1}$ is a $\Lambda_{p, i-1}$-module by virtue of Lemma 11.2;

c) $d_{r}: E_{r}^{p, q} \rightarrow E_{r}^{p+r, q-r+1}$; all differentials $d_{r}$ preserve the dimension of elements induced by the dimension of rings and modules;

d) $E_{2}^{p, 0}=\operatorname{Ext}_{A_{p, i-1}}^{p}\left(\Lambda_{p, i-1}, \Lambda_{p, i-1}\right)$;

e) the spectral sequence $\left(E_{r}, d_{r}\right)$ is a spectral sequence of rings, where the multiplicative structure is induced by the diagonal $\Delta$ of the ring $A_{p, i}$.

The proof of this theorem is more or less standard and is constructed by starting from the double complex corresponding to the central extension $B_{i}$ of the rings $\mathcal{E}_{i}, A_{p, i-1}$. We shall not give it here.

For what follows it will be useful to us to compute $\operatorname{Ext}_{D_{i}}^{* *}\left(Q_{p}[y], Q_{p}[y]\right)$. We note that $A_{p, 1}=D_{1}$, and the calculation of these groups gives certain information about the ring

$$
\operatorname{Ext}_{A^{U}}\left(U^{*}(P), U^{*}(P)\right) \otimes_{Z} Q_{p} .
$$

Lemma 11.3. Let $C$ be a bigraded differential ring over $Q_{p}$, which is associative and is generated by elements

$$
x \in C^{0,2 p^{i}-2}, \quad h_{j} \in C^{1,2 j\left(p^{i}-1\right)}, \quad j \geq 1,
$$


such that:

1) $p h_{j+1}=\left[x, h_{j}\right]=x h_{j}-h_{j} x$

2) $d(x)=p h_{1}$;

3) $\quad d\left(h_{1}\right)=0, \quad d\left(h_{j+1}\right)=\sum_{k=1}^{j}\left(\begin{array}{c}j+1 \\ k\end{array}\right) h_{j+1-k} \cdot h_{k}, \quad j \geq 1$;

4) $d(u v)=(d u) v+(-1)^{i} u(d v)$ where $u \in C^{i}$. Here, $d$ is the differential in the $\operatorname{ring} C^{* *}$.

Then the cohomology ring $H^{* *}(C)$ is canonically isomorphic to the ring $\operatorname{Ext}_{D_{i}}^{* *}\left(Q_{p}\left[y_{i}\right], Q_{p}\left[y_{i}\right]\right)$.

The proof of the lemma consists in constructing a $D_{i}$-free acyclic resolution $\tilde{F}$ of the module $Q_{p}\left[y_{i+1}\right]$, having the form $Q_{p}[y] \cdot F$, where $F$ is a standard resolution over $\mathcal{E}_{i}$ of the trivial module $Q_{p}=\mathcal{E}_{i} / \overline{\mathcal{E}}_{i}$, where $\overline{\mathcal{E}}_{i}$ is the set of elements of positive dimension and $\mathcal{E}_{i}$ is described in Lemma 11.1. The ring $\mathcal{E}_{i}$ has a diagonal, as do $D_{i}$ and $Q_{p}[y]$. Hence the complex $\operatorname{Hom}_{D_{i}}^{* *}\left(\bar{F}, Q_{p}[y]\right)$ is a ring, which coincides exactly, as is easy to verify, with the ring $C$ together with the differential operator $d$. Whence the lemma follows.

From Lemma 11.3 it is easy to derive

Lemma 11.4. a) For $p=2$ the cohomology ring $H^{* *}\left(C \otimes Z_{2}\right)$ is isomorphic to the polynomial algebra $Z_{2}[x] \otimes Z_{2}\left[h_{1}, h_{2}, \ldots, h_{2^{k}}\right]$ with Bokšteřn homomorphism $\beta$ of the following form:

1) $\beta(x)=h_{1}$

2) $\beta\left(h_{2^{k}}\right)=h_{2^{k-1}}^{2}, k \geq 1, x \in H^{0,2\left(2^{i}-1\right)}, h_{2^{k}} \in H^{2^{k+1}\left(2^{i}-1\right)}\left(C \otimes Z_{2}\right)$.

b) For $p>2$ the ring $H^{* *}\left(C \otimes Z_{p}\right)$ is isomorphic to the ring

$$
Z_{p}[x] \otimes \Lambda\left[h_{1}, h_{p}, \ldots, h_{p^{k}}, \ldots\right] \otimes Z_{p}\left(\gamma_{2}, \ldots, \gamma_{k}, \ldots\right),
$$

where

$$
\begin{gathered}
h_{p^{k}} \in H^{1,2 p^{k}\left(p^{i}-1\right)}\left(C_{i} \otimes Z_{p}\right), \\
\gamma_{k} \in H^{2,2 p^{k}\left(p^{i}-1\right)}\left(C_{i} \otimes Z_{p}\right), \\
x \in H^{0,2 p^{i}-2}\left(C_{i} \otimes Z_{p}\right)
\end{gathered}
$$

and the Bokšteŭn homomorphism $\beta$ has the following:

1) $\beta(x)=h_{1}$

2) $\beta\left(h_{p^{k}}\right)=\gamma_{k}, k \geq 1$.

c) The group $\operatorname{Ext}_{D_{i}}^{1, t}\left(Q_{p}\left[y_{i}\right], Q_{p}\left[y_{i}\right]\right)$ is nontrivial for $t=2 p\left(p^{i}-2\right), q \geq 1$, and is isomorphic to the cyclic group $Z_{f(q)}$, where $f(q)-1$ is equal to the largest power of $p$ which divides $q$. We shall denote the generator of the group $\operatorname{Ext}_{D_{i}}^{1,2 q\left(p^{i}-1\right)}\left(Q_{p}[y], Q_{p}[y]\right)$ by $\delta_{q}$.

d) The image of the homomorphism of "reduction modulo $p$,"

$$
\alpha: \operatorname{Ext}^{1,2 q\left(p^{i}-1\right)}\left(Q_{p}\left[y_{i}\right], Q_{p}\left[y_{i}\right]\right) \rightarrow H^{1,2 q\left(p^{i}-1\right)}\left(C_{i} \otimes Z_{p}\right)
$$

is generated by the following elements:

1) $h_{1} x^{q-1}$ for $p>2$ and all $q$,

2) $h_{1} x^{q-1}$ for $q=2$ and $q \equiv 1 \bmod 2$,

3) $h_{1} x^{q-1}+h_{2} x^{q-2}$ for $p=2$ and $q \equiv 0 \bmod 2$. 
e) For all $t>1$, in the groups $H^{t, *}\left(C_{i} \otimes Z_{2}\right)$ the kernel Ker $\beta$ coincides with the image $\operatorname{Im} \beta$. Hence, the homomorphism of reduction modulo $p$,

$$
\alpha_{p}: \operatorname{Ext}_{D_{i}}^{t, *}\left(Q_{p}\left[y_{i}\right], Q_{p}\left[y_{i}\right]\right) \rightarrow H^{t, *}\left(C_{i} \otimes Z_{p}\right)
$$

is an isomorphism on the kernel $\operatorname{Ker} \beta=\operatorname{Im} \beta$ and none of these groups has elements of order $p^{2}$.

The proof of (a) and (b) follows easily from the form of the ring $C$ - in particular, from the fact that $C \otimes Z_{p}$ is commutative, $C$ is obtained from the standard $\mathcal{E}_{i^{-}}$ resolution and $\mathcal{E}_{i} \otimes Z_{p}$ has a system of generators $\left\{l_{i, p^{j}}\right\}$, while

$$
H^{* *}\left(C_{i} \otimes Z_{p}\right)=Z_{p}[x] \otimes \operatorname{Ext}_{\mathcal{E}_{i} \otimes Z_{p}}\left(Z_{p}, Z_{p}\right) .
$$

The structure of the Bokšteĭn homomorphism $\beta$ is derived immediately from Lemma 11.3.

Part (c) follows from the fact that $e_{i, k}^{*}\left(y_{i}^{q}\right)=\sum_{k \geq 1}\left(\begin{array}{l}q \\ k\end{array}\right) p^{k} x^{q-k}$, as was shown in

Lemma 11.1, and from the construction of the standard $\mathcal{E}_{i}$-resolution $F$ for the module $\mathcal{E}_{i} / \overline{\mathcal{E}}_{i}=Q_{p}$ and the differential $d^{*}$ in the complex $\operatorname{Hom}^{*}\left(Q_{p}\left[y_{i}\right] \cdot F, Q_{p}\left[y_{i}\right]\right)$. Namely, we have:

$$
d^{*}\left(x^{q}\right)=\sum_{k \geq 1}\left(\begin{array}{l}
q \\
k
\end{array}\right) p^{k} h_{k} x^{q-k} .
$$

Part (d) is derived from the fact that $\frac{1}{p^{f(q)}} d\left(x^{q}\right) \bmod p$ is equal to $h_{1} x^{q-1}$ for $p>2$ or $p=2, q=2 s+1$, and is equal to $h_{1} x^{q-1}+h_{2} x^{q-2}$ for $p=2, q=2 s$.

We shall now prove part (e). Since the homomorphism $\beta$ is a differential operator, it suffices to show that $H^{t}\left(H^{*}\left(C_{i} \otimes Z_{p}\right), \beta\right)=0$ for $t>1$. The structure of the homomorphism $\beta$ was determined in parts (a) and (b) of Lemma 11.4, and the required fact is easily derived from the usual homological arguments. The lemma is proved.

1. The ring structure in $\operatorname{Ext}_{D_{i}}^{* *}\left(Q_{p}[y], Q_{p}[y]\right)$ completely follows from Lemma 11.4 , since the homomorphism of reduction modulo $p$,

$$
\alpha_{p}: \operatorname{Ext}_{D_{i}}^{* *}\left(Q_{p}[y], Q_{p}[y]\right) \rightarrow H^{* *}\left(C_{i} \otimes Z_{p}\right)
$$

is a monomorphism on $\operatorname{Ker} \beta$ and in dimensions $\geq 2$; hence, from $\alpha_{p}(x y)=0$ it follows that $x y=0$ for elements $x, y$ of positive dimension. The image of the homomorphism $\alpha_{p}\left(\operatorname{Ext}_{D_{i}}^{* *}\right)$ coincides with $\operatorname{Ker} \beta$ in all dimensions $\geq 1$, although Ker $\alpha_{p}$ is nontrivial in dimension 1 [see parts (c) and (d)].

2. The product $\operatorname{Ext}_{D_{i}}^{1, *} \otimes \operatorname{Ext}_{D_{i}}^{1, *}\left(Q_{p}[y], Q_{p}[y]\right)$ is identically equal to zero for $p>2$.

3. A basis for the group $\operatorname{Ext}_{D_{i}}^{2, *}\left(Q_{p}[y], Q_{p}[y]\right)$ is completely given by the set of elements:

a) $\quad \alpha_{k}^{(m)}=\beta\left(h_{k} x^{m}\right), \quad k \geq 1, m \geq 0$ where $p>2$, where

$$
\beta\left(h_{p^{k}} x^{m}\right)=\left(\gamma_{k} x^{m}-m h_{p^{k}} h_{1}\right) \in \operatorname{Ext}_{D_{i}}^{2,\left(p^{k}+2 m\right)\left(p^{i}-1\right)},
$$

b) $\quad \alpha_{k}^{m}=\beta\left(h_{2^{k}} x^{m}\right)=\left(h_{2^{k-1}}^{2} x^{m}+m h_{2^{k}} h_{1} x^{m-1}\right)$ where $p=2, k \geq 2, m \geq 0$.

4. For $p=2$ the product $\operatorname{Ext}_{D_{i}}^{1, *} \otimes \operatorname{Ext}_{D_{i}}^{1, *} \rightarrow \operatorname{Ext}_{D_{i}}^{2, *}$ is defined by the formulas:

$$
\begin{aligned}
& \text { a) } \delta_{2 q+1} \cdot \delta_{2 l+1}=\alpha_{1}^{(2 q+2 l)} \\
& \text { b) } \delta_{2 q+1} \cdot \delta_{2 l}=\alpha_{1}^{(2 q+2 l-1)} \\
& \text { c) } \delta_{2 l} \cdot \delta_{2 m}=\alpha_{1}^{(2 q+2 l-2)}+\alpha_{1}^{(2 q+2 l-4)}
\end{aligned}
$$


In particular, we shall denote the element $\delta_{1}$ by $h \in \operatorname{Ext}^{1,2}$. Hence, from (a) and (b) it follows that

$$
\delta_{2 q+1} \cdot \delta_{m}=h \delta_{2 q+m} \text { for all } q, m .
$$

We note that $D_{1}=A_{p, 1}$ and there is defined a natural homomorphism

$$
\operatorname{Ext}_{D_{1}}^{t, *}\left(Q_{p}\left[y_{1}\right], Q_{p}\left[y_{1}\right]\right) \stackrel{\gamma^{(t)}}{\longrightarrow} \operatorname{Ext}_{A^{U}}^{t, *}\left(U^{*}(P), U^{*}(P)\right) \otimes_{Z} Q_{p}
$$

From Lemma 11.4 and the results of $\S \S 7,8$ is derived the following

Theorem 11.2. a) For $t=1$ the homomorphism $\gamma_{p}^{(1)}$ is a monomorphism.

b) For all $p>2$ the homomorphism $\gamma_{p}^{(1)}$ is an isomorphism.

c) For $p=2$ the homomorphism $\gamma_{p}^{(1)}$ is an isomorphism on the groups Ext $^{1,2 q}$ for $q=2$ and for $q$ odd; for $q=2 s, s \geq 2$, the image of the homomorphism $\gamma_{2}^{(1)}$ nas index 1 or 2 in $\operatorname{Ext}_{A^{U}}^{1,2 q} \otimes_{Z} Q_{2}$ and in fact index 2 for all $q=4 s, s \geq 1$.

d) For all $q=4 s+1$ and $q=4 s+2$ the image $\operatorname{Im} \gamma_{2}^{(1)}$ coincides with the image of the Hopf invariant $q_{1}^{U}\left(\pi_{*}\left(S^{n}\right)\right)=q_{1}^{U}\left(J \pi_{*}(S O)\right)$. For $p>2$ the image $\operatorname{Im} \gamma_{p}^{(1)}$ coincides with the image of the Hopf invariant $q_{1}^{U}\left(\pi_{*}\left(S^{n}\right)\right)=q_{1}^{U}\left(J \pi_{*}(S O)\right)$.

In the formulation of Theorem 11.2 the calculation of the group $\operatorname{Ext}_{A^{U}}^{1,8 k+4} \otimes_{Z} Q_{p}$ not complete - is the homomorphism $\gamma_{2}^{(1)}$ an epimorphism or does $\operatorname{Im} \gamma_{2}^{1}$ have index 2 ?

For the study of this question we shall use the spectral sequence $\left(E_{r}, d_{r}\right)$ described in Theorem 11.1, which converges to the groups $\operatorname{Ext}_{A_{2,2}}\left(\Lambda_{2,2}, \Lambda_{2,2}\right)$. Namely, we must compute the groups $E_{2}^{0,1}$ and the differential

$$
d_{2}: E_{2}^{0,1} \rightarrow E_{2}^{2,0} \approx \operatorname{Ext}_{A_{2,1}}^{2}\left(\Lambda_{2,1}, \Lambda_{2,1}\right)=\operatorname{Ext}_{D_{1}}^{2}\left(Q_{2}\left[y_{1}\right], Q_{2}\left[y_{1}\right]\right)
$$

The groups $\operatorname{Ext}_{D_{1}}^{2}$ were computed in Lemma 11.4 for all $p \geq 2$. We may assume that $y_{1}=\left[C P^{p-1}\right] \in \Omega_{U}$ and

$$
y_{2}=\frac{1}{p}\left(\left[C_{p}^{p^{2}-1}\right]+\lambda\left[C_{p}^{p-1}\right]^{p+1}\right)=\frac{1}{p}\left(x_{2}+\lambda x_{1}^{p+1}\right),
$$

where $x_{i}=\left[C P^{p^{i}-1}\right]$. Moreover, by the integrality of the Todd genus we can set $\lambda=p-1$ and

We have:

$$
y_{2}=\frac{1}{p}\left(x_{2}+(p-1) x_{2}^{p+1}\right), \quad y_{1}=x_{1} \text {. }
$$

$$
\Lambda_{p, 1}=Q_{p}\left[y_{1}\right], \quad \Lambda_{p, 2}=Q_{p}\left[y_{1}, y_{2}\right]
$$

The action of the operation $\Phi \cdot P^{k} \cdot \Phi$ on $\Lambda_{p, 1}$ and $\Lambda_{p, 2}$ is given by the formulas:

$$
\begin{gathered}
\Phi \cdot P^{k} \cdot \Phi\left(x_{1}^{q}\right)=\left(\begin{array}{l}
q \\
k
\end{array}\right) p^{k} x_{1}^{q-k}, \\
\Phi \cdot P^{k} \cdot \Phi\left(x_{2}\right)=\left\{\begin{array}{l}
0, \quad k \neq p, p+1, \\
\left(\begin{array}{c}
p^{2} \\
p
\end{array}\right) x_{1}, k=p, \\
\left(\begin{array}{c}
p^{2} \\
p+1
\end{array}\right), k=p+1 .
\end{array}\right.
\end{gathered}
$$

As a consequence of this we have 
Lemma 11.5. The action of the operators $P^{k}$ on the generators $y_{2}^{q}$ of the ring $\Lambda_{p, 2}$ is given by the following formula:

$$
\left(\Phi P^{k} \Phi\right)^{*}\left(y_{2}^{q}\right)=\sum_{k \geq 1}\left[y_{2}^{q-k} \otimes\left(\sum_{\substack{l_{1}>0 \\
l_{k}>0 \\
\sum l_{i}=k}} P^{l_{1}}\left(y_{2}\right) \circ \cdots \circ P^{l_{k}}\left(y_{2}\right)\right) \circ\left(\begin{array}{l}
q \\
k
\end{array}\right)\right],
$$

where $\left(l_{2}, \ldots, l_{k}\right)$ is an ordered partition of the integer $k$ and

a) $P^{l}\left(y_{2}\right)=\frac{1}{p}\left(P^{l}\left(x_{2}\right)+(p-1) P^{l}\left(x_{1}^{p+1}\right)\right)=(p-1)\left(\begin{array}{c}p+1 \\ l\end{array}\right) p^{l} x_{1}^{p+1-l}$ for $l \neq p$, $p+1$,

b) $P^{p}\left(y_{2}\right)=\frac{1}{p}\left(\left(\begin{array}{c}p^{2} \\ p\end{array}\right)+\left(\begin{array}{c}p+1 \\ p\end{array}\right) p^{p}\right) x_{1}$,

c) $P^{p+1}\left(y_{2}\right)=\frac{1}{p}\left(\left(\begin{array}{c}p^{2} \\ p+1\end{array}\right)+p^{p+1}\right)$.

We note that $P^{1}\left(y_{2}\right)$ is divisible by $p$ for $1 \neq p$ and $P^{p}\left(y_{2}\right)$ is not divisible by $p$.

Now we can describe the action of the ring $A_{p, 1}$ on $\Gamma^{1} \otimes \Lambda_{p, 1}$, where $\Gamma^{t, s}=\operatorname{Ext}_{D_{2}}^{t,-s}\left(Q_{p}\left[y_{2}\right], Q_{p}\left[y_{2}\right]\right)$ and $\lambda_{p, 1}=Q_{p}\left[y_{1}\right]=Q_{p}\left[x_{1}\right], x_{1}=\left[C P^{p-1}\right]$. The groups $\Gamma^{1, *}$ were computed in Lemma 11.4, part (c). The generator of the group $\operatorname{Ext}^{1, q\left(p^{2}-1\right)}\left(Q_{p}\left[y_{2}\right], Q_{p}\left[y_{2}\right]\right)$ is obtained as $d^{*}\left(x^{q}\right) / p^{f(q)}$ in the complex $\operatorname{Hom}_{D_{2}}^{*}\left(F, Q_{p}\left[\gamma_{2}\right]\right)$ where $F$ is a $D_{2}$-free acyclic resolution of the module $Q_{p}\left[y_{2}\right], x^{q} \in \operatorname{Hom}^{*}\left(D_{2}, Q_{p}\left[y_{2}\right]\right)$ is an element such that $x^{q}(1)=y_{2}^{q}, f(q)-1$ is the maximal power of $p$ which divides $q$, and $d^{*}$ is the differential in the complex $\operatorname{Hom}_{D_{2}}^{*}\left(F, Q_{p}\left[y_{2}\right]\right)$.

We set

$$
P^{k}\left(y_{2}^{q}\right)=\sum_{k=1}^{q} y_{2}^{q-k} \otimes a_{k}
$$

where

$$
a_{k}=\sum_{\substack{\sum_{\begin{subarray}{c}{i \\
l_{i}>0} }}} \\
{l_{i}}\end{subarray}} P^{l}\left(y_{2}\right) \ldots P^{l_{s}}\left(y_{2}\right)\left(\begin{array}{l}
q \\
k
\end{array}\right)
$$

by virtue of Lemma 11.5 and $a_{k} \in Q_{p}\left[y_{1}\right], a_{k}=\lambda^{k} y_{1}^{s_{k}}$. From what has been said it is easy to derive

Lemma 11.6. The action of the ring $A_{p, 1}=D_{1}$ on $\Gamma^{1} \otimes Q_{p}\left[y_{2}\right]$ is described in the following fashion:

$$
P^{k}\left(\alpha_{q}\right)=\sum_{k=1}^{q-1} \alpha_{q-k} \otimes p^{f(q-k)-f(q)} \circ a_{k}
$$

where

$$
\alpha_{q} \in \Gamma^{1,-2 q\left(p^{2}-1\right)}=\operatorname{Ext}_{D_{2}}^{1,2 q\left(p^{2}-1\right)}\left(Q_{p}\left[y_{2}\right], Q_{p}\left[y_{2}\right]\right)
$$

are generators (their orders are $p^{f(q)}$ ) and $a_{k} \in Q_{p}\left[y_{1}\right]$ is described in Lemma 11.4.

Lemma 11.6 follows easily from Lemma 11.4 and the definition of the generators $\alpha_{q}=d^{*} x^{q} / p^{f(q)}$, where $x^{q} \in \operatorname{Hom}^{*}\left(F, Q_{p}\left[y_{2}\right]\right)$ is such that $x^{q}(1)=y_{2}^{q} \in Q_{p}\left[y_{2}\right]$. Further, we compute $\operatorname{Hom}_{A_{p, 1}}^{*}\left(\Lambda_{p, 2}, \Gamma^{1} \otimes \Lambda_{p, 1}\right)=E_{2}^{0,1}$ in the spectral sequence $\left(E_{2}, d_{2}\right)$ of Theorem 11.1, which converges to $\operatorname{Ext}_{A_{p, 2}}^{* *}\left(\Lambda_{p, 2}, \Lambda_{p, 2}\right)$; here $A_{p, 1}=D_{1}$ and $\Lambda_{p, 1}=Q_{p}\left[y_{1}\right]$. 
We have the following

Lemma 11.7. The groups $\operatorname{Hom}_{A_{p, 1}}^{t}\left(\Lambda_{p, 1}, \Gamma^{1} \otimes \Lambda_{p, 1}\right)$ are spanned by generators $\kappa_{i, q}$ of dimension $2 p^{i}\left(p^{2}-1\right)+2 q(p-1)$ for all $i \geq 0, q \geq 0$, where the order of the generator $\kappa_{i, q}$ is $p$.

The proof of the lemma follows easily from Lemma 11.5 and 11.6 by direct calculation.

Since $\operatorname{Hom}_{A_{p, 1}}^{*}\left(\Lambda_{p, 1}, \Gamma^{1} \otimes \Lambda_{p, 1}\right)=E_{2}^{0,1}$, our problem is to calculate $d_{2}: E_{2}^{0,1} \rightarrow$ $E_{2}^{2,0}=\operatorname{Ext}_{A_{p, 1}}^{2}\left(\Lambda_{p, 1}, \Lambda_{p, 1}\right)$, where the latter groups are computed in Lemma 11.4 and in the conclusions drawn from it.

Direct calculation proves

Lemma 11.8. The differential $d_{2}: E_{2}^{0,1} \rightarrow E_{2}^{2,0}$ of the spectral sequence $\left(E_{r}, d_{r}\right)$ converging to $\operatorname{Ext}_{A_{p, 2}}\left(\Lambda_{p, 2}, \Lambda_{p, 2}\right)$ is given by the following formula:

$$
d_{2}\left(\kappa_{i, t}\right)=\beta\left(h_{p^{i+1}} x^{p^{i}+t}+h_{p^{i}} x^{p^{i}+t}\right), \quad i \geq 0, \quad t \geq 0,
$$

where $h_{p^{i}}$ and $x$ are in the notation of Lemma 11.4, and $\beta$ is the Bokštern homomorphism $H^{* *}(C) \rightarrow \operatorname{Ext}_{D_{1}}\left(Q_{p}\left[x_{1}\right]\right)$ described in Lemma 11.4.

From Lemma 11.8 follows the important

Corollary 11.1. a) For $p>2$, the kernel Ker $d_{2} \mid E_{2}^{0,1}$ is trivial;

b) For $p=2$, the kernel Ker $d_{2} \mid E_{2}^{0,1}$ is generated by elements

$$
\kappa_{0,2 t+1} \in \operatorname{Hom}_{A_{p, 1}}^{4 t+8}\left(\Lambda_{p, 1} ; \Gamma^{1} \otimes \Lambda_{p, 1}\right), \quad t \geq 0 .
$$

Hence, the image of the homomorphism

$$
\operatorname{Ext}_{A_{p, 1}}^{1,4 t+8}\left(\Lambda_{p, 1} ; \Lambda_{p, 1}\right) \rightarrow \operatorname{Ext}_{p, 2}^{1,4 t+8}\left(\Lambda_{p, 2} ; \Lambda_{p, 2}\right)
$$

has index 2 for all $t \geq 0$.

Parts (a) and (b) of the corollary are derived in an obvious way from the structure of the homomorphism $\beta$, which was completely described in Lemma 11.4. The sharp distinction between the cases $p=2$ and $p>2$ is explained by the fact that for $p>2$ we have $h_{1}^{2}=0$ and $\beta\left(h_{1} x^{s}\right)=0$ for all $s \geq 0$, while for $p=2, \beta\left(h_{1} x^{2 s+1}\right) \neq 0$.

Comparing part (b) of Corollary 11.1 with Theorem 11.2, we obtain the following result.

Theorem 11.3. a) In all dimensions $t \neq 4$, the order of the cyclic group $\operatorname{Ext}_{A^{U}}^{1, t}\left(U^{*}(P), U^{*}(P)\right)$ coincides exactly with the order of the group $\operatorname{Ext}_{A_{\Psi}^{k}}^{1, t}\left(K^{*}(P), K^{*}(P)\right)$, and this isomorphism is induced by the Riemann-Roch functor $\lambda$.

b) The Hopf invariant

$$
q_{1}: \pi_{n+t-1}\left(S^{n}\right) \rightarrow \operatorname{Ext}_{A^{U}}^{1, t}\left(U^{*}(P), U^{*}(P)\right)
$$

is an epimorphism for $t=8 k, t=8 k+2$ and $t=4$, and the image $\operatorname{Im} q_{1}$ has index 2 in $\operatorname{Ext}_{A^{U}}^{1, t}$, for $t=8 k+6, k \geq 0$, and $t=8 k+4, k \geq 1$.

Corollary 11.2. The generators $\alpha_{q}$ of the groups $\operatorname{Ext}_{A^{U}}^{1,2 q}\left(U^{*}(P), U^{*}(P)\right)$ are cycles for all Adams differentials $d_{i}$ for $q=4 s, 4 s+1, s \geq 0$, and $q=2$, and are not 
cycles for all differentials for $q=4 s-1,4 s+2, s \geq 1$ (the elements $2 \alpha_{q}$ are cycles for all differentials $)^{4}$.

Supplementary remark. It is possible in all dimensions to prove the formula $d_{3}\left(\alpha_{q}\right)=h^{3} \cdot \alpha_{q-2}$ for $q=4 s-1,4 s+2, s \geq 1$, where $h=\alpha_{1} \in \operatorname{Ext}_{A^{U}}^{1,2}$. In particular, for $q=4 s+2$ this follows from the fact that $h^{3} \alpha_{q-2} \neq 0$ in $\operatorname{Ext}_{A^{U}}^{4}$, while at the same time $\alpha_{q-2}$ is realized by the image of the $J$-homomorphism, and we must have in $E_{\infty}$ that $h^{3} \alpha_{q-2}=0$.

\section{$\S$ 12. The Adams speCtral Sequence and double Complexes. COMPARISON OF DIFFERENT COHOMOLOGY THEORIES}

We assume that there is given a complex $Y=Y_{-1} \in \vec{S}$ and a filtration

$$
Y \leftarrow Y_{0} \leftarrow Y_{1} \leftarrow \cdots \leftarrow Y_{i} \leftarrow \ldots,
$$

where the complex of $A^{X}$-modules $\left\{X^{*}\left(Y_{i}, Y_{i+1}\right)=M_{i}\right\}$

$$
M=\left\{M_{0} \stackrel{d}{\leftarrow} M_{1} \leftarrow M_{2} \leftarrow \ldots \stackrel{d}{\leftarrow} M_{i} \leftarrow \ldots\right\}
$$

is acyclic in the sense that $H_{i}(M)=0, i>0$, and $H_{0}(M)=X^{*}(Y)$. The modules $M_{i}$ are not assumed to be projective. In the usual way a double complex of $A^{X}$-free modules $N=\left(N_{i j}\right)$ is constructed.

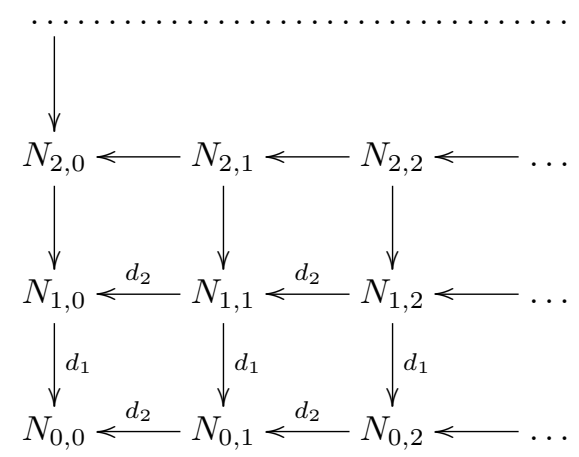

such that (a) $d_{1} d_{2}=-d_{2} d_{1}$; (b) $\left\{\rightarrow \cdots \rightarrow N_{i j} \stackrel{d_{1}}{\rightarrow} N_{i-1, j} \rightarrow \ldots\right\}$ for all $j$ is an $A^{X}$-free acyclic resolution of the module $M_{j}$; (c) if $Q_{k}=\sum_{j+i=k} N_{i j}$ and $d=$ $d_{1}+d_{2}: Q_{k} \rightarrow Q_{k-1}$, then the complex $\left\{Q_{k} \stackrel{d}{\rightarrow} Q_{k-1} \rightarrow \ldots\right\}$ is an $A^{X}$-free acyclic resolution of the module $X^{*}(Y)$; (d) the complex $N_{i}=\left\{\rightarrow N_{i, j} \stackrel{d_{2}}{\longrightarrow} N_{i, j-1} \rightarrow \ldots\right\}$ is such that $H_{k}\left(N_{i}\right)=0$ for $k>0, H_{0}\left(N_{i}\right)$ is a free $A^{X}$-module and the complex $\left\{\ldots H_{0}\left(N_{k}\right) \stackrel{d_{1}}{\longrightarrow} H_{0}\left(N_{k-1}\right) \rightarrow \ldots\right\}$ represents an $A^{X}$-free acyclic resolution of the module $X^{*}(Y)$.

As usual, there arises a spectral sequence of the double complex $\left(E_{r}^{t, q}, d_{r}\right)$, where

$$
d_{r}: E_{r}^{t, q} \rightarrow E_{r}^{t+r, q-r+1}
$$

and

$$
E_{2}^{t, q}=\operatorname{Ext}_{A^{X}}^{t}\left(M_{q}, L\right)
$$

\footnotetext{
${ }^{4}$ We take this opportunity to note the small computational error in parts (3) and (4) of Theorem 5 of the author's paper [19], which is completely corrected in Theorem 11.3 and Corollary 11.2 of the present paper.
} 
with $L$ an arbitrary $A^{X}$-module; this spectral sequence converges to $\operatorname{Ext}_{A^{X}}\left(X^{*}(Y), L\right)$.

Definition 12.1. By a geometric realization of the double complex $N=\left(N_{i j}\right)$ in the category $\vec{S}$ or $\vec{S} \otimes_{Z} Q_{p}$ is meant a set of objects $\left(Z_{i j}\right), i \geq-1, j \geq-1$, and morphisms

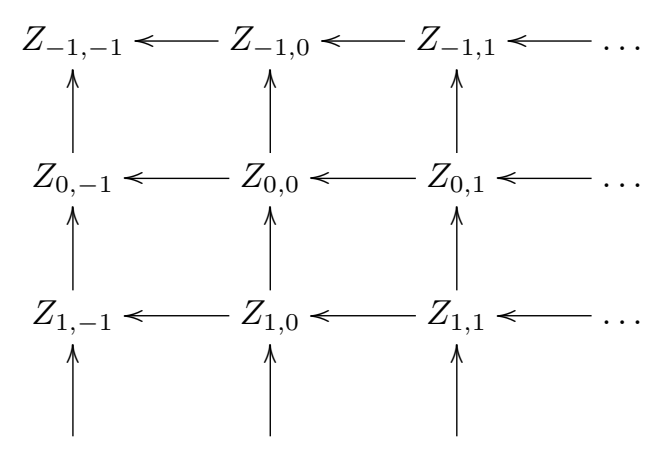

with the following properties:

a) $Z_{-1,-1}=Y, Y_{i}=Z_{-1, i}$ and the filtration $Z_{-1,-1} \leftarrow Z_{-1,0} \ldots$ coincides with the filtration $Y \leftarrow Y_{0} \leftarrow \cdots \leftarrow Y_{i} \leftarrow \ldots$

b) The filtration

$$
Y_{i} / Y_{i+1}=Z_{-1, i} / Z_{-1, i+1} \leftarrow Z_{0, i} / Z_{0, i+1} \leftarrow Z_{1, i} / Z_{1, i+1} \leftarrow \ldots
$$

represents a geometric realization of the $A^{X}$-free resolution of the module $X^{*}\left(Y_{i} / Y_{i+1}\right)=M_{i}$,

$$
\left\{M_{i} \stackrel{\varepsilon}{\longleftarrow} N_{0, i} \stackrel{d_{1}}{\longleftarrow} N_{1, i} \stackrel{d_{1}}{\longleftarrow} \ldots\right\}
$$

and hence $X^{*}\left(Z_{k, i} / Z_{k, i+1} \cup Z_{k+1, i}\right)=N_{k, i}$.

c) The differentials $d_{1}: N_{k, i} \rightarrow N_{k-1, i}$ and $d_{2}: N_{k, i} \rightarrow N_{k, i-1}$ coincide with the natural homomorphisms

$$
\begin{aligned}
& X^{*}\left(Z_{k, i} / Z_{k, i+1} \cup Z_{k+1, i}\right) \stackrel{d_{1}}{\longrightarrow} X^{*}\left(Z_{k-1, i} / Z_{k, i} \cup Z_{k-1, i+1}\right), \\
& X^{*}\left(Z_{k, i} / Z_{k, i+1} \cup Z_{k+1, i}\right) \stackrel{d_{2}}{\longrightarrow} X^{*}\left(Z_{k, i-1} / Z_{k, i} \cup Z_{k+1, i-1}\right) .
\end{aligned}
$$

We make some deductions fromi the properties of the geometric realization of a double complex:

1. The filtration $Z_{-1,-1}=Y \leftarrow Z_{0,-1} \leftarrow Z_{1,-1} \leftarrow \cdots \leftarrow Z_{i,-1} \leftarrow \ldots$ represents the geometric realization of the $A^{X}$-free resolution $\left\{H^{*}\left(N_{0}\right) \stackrel{d_{2}}{\longleftarrow} H^{*}\left(N_{1}\right) \leftarrow \ldots\right\}$.

2. The filtration $Y \leftarrow Z_{-1,0} \cup Z_{0,-1} \leftarrow \cdots \bigcup_{i+j=k-1} Z_{i, j} \leftarrow \ldots$ represents the geometric realization of the $A^{X}$-free resolution

$$
X^{*}(Y) \stackrel{\varepsilon}{\leftarrow} N_{0,0} \stackrel{d}{\leftarrow} N_{0,1}+N_{1,0} \leftarrow \cdots \leftarrow \sum_{i+j=k} N_{i, j},
$$

where $d=d_{1}+d_{2}$.

3. The double complex $(Z)$ defines two Adams spectral sequences:

a) the Adams spectral sequence $E_{r, X}$ in the theory $X^{*}$, induced by the filtration

$$
Y \leftarrow Z_{0,-1} \cup Z_{-1,0} \leftarrow \cdots \leftarrow \bigcup_{i+j=k-1} Z_{i, j} \leftarrow \cdots
$$

with term $E_{2}^{k}=\operatorname{Ext}_{A^{X}}^{k}\left(X^{*}(Y), X^{*}(K)\right)$ for any $K \in S$; 
b) the spectral sequence $\bar{E}_{r}$ of the filtration

$$
Y \leftarrow Y_{0} \leftarrow Y_{1} \leftarrow \cdots \leftarrow Y_{i} \leftarrow \ldots
$$

with term $\bar{E}_{1}=\left\{\operatorname{Hom}^{*}\left(K, Y_{i} / Y_{i+1}\right)\right\}$.

In view of the presence of the double filtration $\left(Z_{i j}\right)$ of the complex $Y$ in all terms of both Adams spectral sequences there arises yet another filtration: in the first case it is equal to $\phi(x), x \in E_{r}^{k}$, where $\phi(x)$ coincides in $E_{2}^{k}$ with the filtration in $\operatorname{Ext}_{A^{X}}^{k}\left(X^{*}(Y), X^{*}(K)\right)$ induced by the non-free resolution $X^{*}(Y) \leftarrow M_{0} \leftarrow M_{1} \leftarrow$ $\ldots$, and in $E_{\infty}^{k}$ is induced by the geometric filtration

$$
\bigcup_{\substack{i+j=k-1 \\ i \leq k}} Z_{i, j} \supset \cdots \supset \bigcup_{\substack{i+j=k-1 \\ i \leq k-\phi(x)}} Z_{i, j} \supset \cdots \supset Z_{-1, k} .
$$

c) For the second Adams spectral sequence the filtration in $\bar{E}_{r}^{k}$ and $\bar{E}_{\infty}^{k}$ is induced by the geometric filtration

$$
Z_{-1, k} \supset Z_{0, k} \supset \cdots \supset Z_{s, k} \supset \ldots
$$

We shall denote it by $\Psi(y), y \in \bar{E}_{r}$.

In addition, each of the indicated spectral sequences defines in the groups of homotopy classes of mappings $\operatorname{Hom}^{*}(K, Y)$ the usual filtration $i(x)$, whose corresponding index $i$ is such that the element $x \in \operatorname{Hom}^{*}(K, Y)$ is nontrivial in $E_{\infty}^{i}$ and trivial in $E_{\infty}^{j}$ for $j>i$. For the Adams spectral sequence of the theory $X^{*}$ we shall denote this filtration by $i_{X}$. We have the double filtration $\left[i_{X}(x), \phi(x)\right]$ where $x \in \operatorname{Hom}^{*}(K, Y), \phi(x) \leq i_{x}(x)$.

The second Adams spectral sequence for $\operatorname{Hom}^{*}(K, Y)$, induced by the filtration

$$
Y \leftarrow Y_{0} \leftarrow Y_{1} \leftarrow \cdots \leftarrow Y_{1} \leftarrow \ldots
$$

also induces a double filtration in $\operatorname{Hom}^{*}(K, Y):[i(x), \Psi(x)]$.

From the construction of the double complex it is obvious that we have

Lemma 12.1. The filtrations described above are related by

$$
i(x) \leq \phi(x) \leq i_{X}(x) \leq i(x)+\Psi(x)
$$

for all $x \in \operatorname{Hom}^{*}(K, Y)$ in the presence of a geometric realization of the double complex defining both Adams spectral sequences.

By standard methods one proves

Lemma 12.2. If $X^{*}$ is the theory of $Z_{p}$-cohomology, then for any acyclic filtration $Y=Y_{-1} \leftarrow Y_{0} \leftarrow Y_{1} \leftarrow \ldots$ there exists a geometrically realizable A-free double complex $(Z)$, where $A$ is the ordinary Steenrod algebra.

The proof of this lemma is obtained easily by the methods of [1].

The most important example which we consider here is the theory of cobordism in the category $S \otimes_{Z} Q_{p}$ :

a) $Y \in D_{p}$, i.e., $H^{*}\left(Y, Q_{p}\right)$ has no torsion.

b) $X=H^{*}\left(, Z_{p}\right), A^{X}=A$.

c) The filtration $Y \supset Y_{0} \supset Y_{1} \supset \ldots$ is an acyclic free filtration in the theory $U^{*} \otimes_{Z} Q_{p}$ or in the theory $U_{p}^{*} \subset U^{*} \otimes_{Z} Q_{p}$. By virtue of the exactness of the functor $U_{p}^{*} \rightarrow H^{*}\left(, Z_{p}\right)$ in the category $D_{p}$, the filtration $Y \supset Y_{0} \supset \ldots$ is also acyclic (although not free) in the theory $X=H^{*}\left(, Z_{p}\right)$.

In this example, the filtration $i(x)$ is a homotopy invariant, with $i(x)=i_{U_{p}}(x)$, where $U_{p}^{*}$ is cobordism theory. Moreover, we have 
Lemma 12.3. a) All the filtrations $i_{X}, i_{U_{p}}, \phi, \Psi$, for $X^{*}=H^{*}\left(, Z_{p}\right)$, a $U_{p}^{*}$ free acyclic filtration $Y \supset Y_{0} \supset Y_{1} \supset \ldots$ in the category $S \otimes_{Z} Q_{p}$ and any $X^{*}$-free acyclic double complex $(Z)$ are homotopy invariants of $Y$, and we have the following inequalities:

$$
i_{U_{p}^{*}}(x) \leq \phi(x) \leq i_{H^{*}\left(, Z_{p}\right)}(x) \leq i_{U_{p}^{*}}(x)+\Psi(x),
$$

where $i=i_{U_{p}^{*}}, i_{X}=i_{H^{*}(, Z)}$ are the respective filtrations in the theories $U^{*} \otimes_{Z} Q_{p}$ and $H^{*}\left(, Z_{p}^{p}\right)$.

b) The second Adams spectral sequence $E_{r}$ coincides in this case with the Adams spectral sequence in the theory $U^{*} \otimes_{Z} Q_{p}$ for $r \geq 2$.

c) Both Adams spectral sequences $E_{r}$ in the theories $X^{*}=H^{*}\left(, Z_{p}\right)$ and $U^{*} \otimes_{Z}$ $Q_{p}\left(\right.$ or $\left.U_{p}^{*}\right)$ in our case preserve, respectively, the filtrations $\phi$ and $\Phi$.

d) The Adams spectral sequence in the theory $H^{*}\left(, Z_{p}\right)$ is such that each differential $d_{r}$ for $r \geq 2$ raises the filtration $\phi$ at least by 1 , i.e.,

$$
\phi\left(d_{r} y\right) \geq \phi(y)+1, \quad y \in E_{r} .
$$

For the proof of (a) we note that the $U_{p}^{*}$-filtration $Y \supset Y_{0} \supset Y_{1} \supset \ldots$ depends functorially on the $U_{p}$-free resolution and is uniquely determined by it. For a fixed $U_{p}$-filtration the same thing is true with respect to the double complex $N$ and the double filtration $(Z)$ defined by it. Parts (b) and (c) are obvious. Part (d) follows immediately from the fact that the complex $Y_{i} / Y_{i+1}$ is a direct sum of spectra $M_{p}$ of the theory $U_{p}^{*}$ up to suspension. For such objects the Adams spectral sequence has zero differentials for $r \geq 2$, as was proved by Milnor and the author $[15,17,18]$.

The lemma is proved.

We now consider the graded ring $\Lambda_{p} \subset \Omega_{U} \otimes_{Z} Q_{p}$, where $\Lambda_{p}=Q_{p}\left[x_{1}, \ldots, x_{i}, \ldots\right]$, $\operatorname{dim} x_{i}=2 p^{i}-2$. The ring $\Lambda_{p}$ is a local ring: it has a unique maximal ideal $m \subset \Lambda_{p}$ such that $\Lambda_{p} / m=Z_{p}$. Hence the bigraded ring $\bar{\Lambda}_{p}=\sum_{i=0} m^{i} / m^{i+1}$ is an algebra over $Z_{p}$, and $\bar{\Lambda}_{p}=Z_{p}\left[h_{0}, h_{1}, \ldots, h_{i}, \ldots\right]$, where $h_{0}$ is associated with multiplication by $p$ and $\operatorname{dim} h_{i}=\left(1,2 p^{i}-1\right)$, i.e., $h_{i} \in m / m^{2}$. Clearly $\bigcap_{i} m^{i}=0$ and, by $[15,17,18]$, we have:

$$
\bar{\Lambda}_{p}=\operatorname{Ext}_{A}^{* *}\left(H^{*}\left(M_{p}, Z_{p}\right), Z_{p}\right) .
$$

As was established in $\S 11$, the action of the ring $A_{p}^{U}$ on $\Lambda_{p}=U_{p}^{*}(P)$ preserves the filtration generated by the maximal ideal $m$. Hence it defines an action on $\bar{\Lambda}_{p}$, which is described as follows:

1) the action of $\Lambda_{p}$ on $\bar{\Lambda}_{p}$ is defined by multiplication;

2) the action of $P^{k}$ on $\bar{\Lambda}_{p}$ is defined so that

$$
P^{p^{i}}\left(h_{i}\right)=h_{i-1} \text { and } P^{j}\left(h_{0}\right)=0, \quad j \geq 1, \quad P^{k}(a b)=\sum_{l+s=k} P^{l}(a) P^{s}(b) .
$$

We consider the $\operatorname{ring} \bar{A}$ associated to $A_{p}^{U}$ by the filtration $A_{p}^{U} \supset m A_{p}^{U} \supset \cdots \supset$ $m^{i} A_{p}^{U} \supset \ldots$. We note that in the ordinary Steenrod algebra $A$ there is a normal (exterior) subalgebra $Q \subset A, Q=\Lambda\left(Q_{0}, \ldots, Q_{i}, \ldots\right)$, $\operatorname{dim} Q_{i}=2 p^{i}-1$, such that $A / / Q$ is isomorphic to the quotient $A / \beta A \cup A \beta$ and $\operatorname{Ext}_{A}\left(H^{*}\left(M_{p}, Z_{p}\right)\right)=$ $\operatorname{Ext}_{Q}\left(Z_{p}, Z_{p}\right)=\bar{\Lambda}_{p}=Z_{p}\left[h_{0}, h_{1}, \ldots, h_{i}, \ldots\right]$.

From the results of $\S 11$ and the structure of the Steenrod algebra $A$ follows

Lemma 12.4. The algebra $\bar{A}$ associated to the ring $A_{p}^{U}$ is isomorphic to $\left(\bar{\Lambda}_{p}\right.$. $A / / Q)^{\wedge}$, where the commutation law ah $=\sum_{i} \bar{a}_{i}^{*}(h) \overline{\bar{a}}_{i}$ is given by the action of 
$A / / Q$ on $\bar{\Lambda}_{p}$ defined by the formulas $P^{p^{r}}\left(h_{r}\right)=h_{r-1}, r \geq 1, P^{k}\left(h_{0}\right)=0$ for $k>0$, and $\Delta a=\sum_{i} \bar{a}_{i} \otimes \overline{\bar{a}}_{i}$, where $\Delta: A / / Q \rightarrow A / / Q \otimes A / / Q$ is the diagonal and $P^{k}$ is the ordinary Steenrod power.

We note now the following identity:

$$
\operatorname{Ext}_{\bar{A}}^{s}\left(\bar{\Lambda}_{p}, \bar{\Lambda}_{p}^{t}\right)=\operatorname{Ext}_{A / / Q}^{s}\left(Z_{p}, \bar{\Lambda}_{p}^{t}\right)=\operatorname{Ext}_{A / / Q}^{s}\left(Z_{p}, \operatorname{Ext}_{Q}^{t}\left(Z_{p}, Z_{p}\right)\right)
$$

(here, $t$ is the dimension in $\bar{\Lambda}_{p}$ defined by the filtration $\bar{\Lambda}_{p}^{t}=m^{t} / m^{t+1}$ ). Moreover, if $Y \subset D_{p}$, then for $L^{p}=U_{p}^{*}(Y)$ and $M=H^{*}\left(Y, Z_{p}\right)=L / m L$ we have:

a) $M$ is an $A / / D$-module;

b) there exists the identity

$$
\operatorname{Ext}_{\bar{A}}^{s}\left(\bar{L}, \bar{\Lambda}_{p}^{t}\right)=\operatorname{Ext}_{A / / Q}^{s}\left(M, \operatorname{Ext}_{Q}^{t}\left(Z_{p}, Z_{p}\right)\right),
$$

where $\bar{L}=\sum m^{i} L / m^{i+1} L$ is an $\bar{A}$-module and, clearly, a $\bar{\Lambda}_{p}$-free module.

Two spectral sequences $\left(\tilde{E}_{r}\right),\left(\tilde{\tilde{E}}_{r}\right)$ arise, both with the term

$$
\tilde{E}_{2}=\tilde{\tilde{E}}_{2}=\operatorname{Ext}_{A / / Q}\left(M, \operatorname{Ext}_{Q}\left(Z_{p}, Z_{p}\right)\right) .
$$

These sequences have the following properties:

1) In the first, which converges to $\operatorname{Ext}_{A}\left(M, Z_{p}\right)$, we have

$$
\tilde{d}_{r}: \tilde{E}_{r}^{s, t} \rightarrow \tilde{E}_{r}^{s+r, t-r+1} .
$$

2) In the second, which is induced by the filtrations in $\Lambda_{p}, A_{p}^{U}, L$ and which converges to $\operatorname{Ext}_{A_{p}^{U}}\left(L, \Lambda_{p}\right)$, we have:

$$
\tilde{\tilde{d}}_{r}: \tilde{\tilde{E}}_{r}^{s, t} \rightarrow \tilde{\tilde{E}}_{r}^{s+1, t+r-1} .
$$

3) $\tilde{d}_{1}=\tilde{\tilde{d}}_{1}$ and $\tilde{E}_{2}^{s, t}=\tilde{\tilde{E}}_{2}^{s, t}=\operatorname{Ext}_{A / / Q}^{s}\left(M, \operatorname{Ext}_{Q}^{t}\left(Z_{p}, Z_{p}\right)\right)$.

4) In both spectral sequences there is yet another grading $\tilde{E}_{r}^{s, t}=\sum_{q} \tilde{E}_{r}^{s, t, q}$ and $\tilde{\tilde{E}}_{r}^{s, t}=\sum_{q} \tilde{\tilde{E}}_{r}^{s, t, q}$, induced by the dimensions in all modules and algebras which appear, and connected to the spectral sequences as follows:

a) the third grading $q$ is preserved by all differentials $\tilde{d}_{r}$ of the spectral sequence $\tilde{E}_{r}$ which converges to $\operatorname{Ext}_{A}\left(M, Z_{p}\right)$;

b) since $\sum_{t-q=m} \bar{\Lambda}_{p}^{t, q}$ is associated to $\Lambda_{p}^{m}$, the third grading $q$ in the second spectral sequence $\tilde{\tilde{E}}_{r}$, which converges to $\operatorname{Ext}_{A_{p}^{U}}\left(L, \Lambda_{p}\right)$, is increased by $r-1$ by the differential $\tilde{\tilde{d}}_{r}$ :

$$
\begin{gathered}
\tilde{d}_{r}: \tilde{E}_{r}^{s, t, q} \rightarrow \tilde{E}_{r}^{s+r, t-r+1, q}, \\
\tilde{\tilde{d}}_{r}: \tilde{\tilde{E}}_{r}^{s, t, q} \rightarrow \tilde{\tilde{E}}_{r}^{s+1, t+r-1, q+r-1} .
\end{gathered}
$$

5). a) The group $\sum_{s+t=m} \tilde{E}_{\infty}^{s, t, q}$ is associated with

$$
\operatorname{Ext}_{A}^{m, q}\left(M, Z_{p}\right)=\operatorname{Ext}_{A}^{m, q}\left(H^{*}\left(Y, Z_{p}\right), Z_{p}\right) .
$$

b) The group $\sum_{q-t=l} \tilde{\tilde{E}}_{\infty}^{s, t, q}$ is associated with

$$
\operatorname{Ext}_{A_{p}^{U}}^{s, t}\left(L, \Lambda_{p}\right)=\operatorname{Ext}_{A^{U}}^{s, l}\left(U^{*}(Y), U^{*}(P)\right) \otimes_{Z} Q_{p},
$$

where $L=U^{*}(Y), \Lambda_{p}=U_{p}^{*}(P)$. 
Thus, in the groups

$$
E_{2}^{s, t, q}=\operatorname{Ext}_{A / / Q}^{s, q}\left(M, \operatorname{Ext}_{Q}^{t}\left(Z_{p}, Z_{p}\right)\right)
$$

we have two "dimensions": $(m, q)=(s+t, q)$ is the "cohomological" and $(s, q-t)=$ $(s, l)$ is the "unitary" (in $U$-cobordism). The "geometric" dimension (of the homotopy groups) is equal to $q-m=l-s=q-s-t$.

We note the important fact: the dimension of the element $\tilde{d}_{r}(y)$ for the element $y$ of "unitary" dimension $(s, l)$ is equal to $(s+r, l+r-1)$, where $l=q-t$; and, conversely, $\tilde{\tilde{d}}_{r}(y)$ of an element of "cohomological" dimension $(m, q)$ has "cohomological" dimension $(m+r, q+r-1), m=s+t$. This means that both these spectral sequences have the form of the Adams spectral sequence, although they are defined purely algebraically by the $\operatorname{ring} A_{p}^{U}$.

Up to this point there has been no difference between $p=2$ and $p>2$, if we speak of the results if this section. However, the following theorem shows the comparative simplicity of the case $p>2$.

Theorem 12.1. For any $p>2$ and complex $Y \subset D_{p}$, the spectral sequence $\left(\tilde{E}_{r}, \tilde{d}_{r}\right)$ has all differentials $\tilde{d}_{r}=0$ for $r \geq 2$. The groups

$$
\sum_{s+t=m} \tilde{E}_{2}^{s, t, q}=\sum_{s+t=m} \operatorname{Ext}_{A / / Q}^{s, q}\left(M, \operatorname{Ext}_{Q}^{t}\left(Z_{p}, Z_{p}\right)\right)
$$

are isomorphic to $\operatorname{Ext}_{A}^{m, q}\left(M, Z_{p}\right)$, where $M=H^{*}\left(Y, Z_{p}\right)$,

$$
\operatorname{Ext}_{Q}^{*}\left(Z_{p}, Z_{p}\right)=Z_{p}\left[h_{0}, \ldots, h_{i}, \ldots\right], \quad \operatorname{dim} h_{i}=\left(1,2 p^{i}-1\right),
$$

and the algebra $A / / Q$ generated by the Steenrod powers $P^{p^{i}}$ acts on $\operatorname{Ext}_{Q}\left(Z_{p}, Z_{p}\right)$ in the following way: $P^{p^{i}}\left(h_{i+1}\right)=h_{i}, P^{k}\left(h_{0}\right)=0$ for $k>0$, and $P^{k}(x y)=$ $\sum_{i+j=k} P^{i}(x) P^{j}(y)$.

From Theorem 12.1 follows

Corollary 12.1. For any complex $Y \in D_{p}$, where $p>2$, there is defined an "algebraic Adams spectral sequence" $\left(\tilde{\tilde{E}}_{r}, \tilde{\tilde{d}}_{r}\right)$, where $\tilde{\tilde{E}}_{2}^{s, t, q}=\operatorname{Ext}_{A / / Q}^{s, q}\left(M, \operatorname{Ext}_{Q}^{t}\left(Z_{p}, Z_{p}\right)\right)$, the group $\sum_{s+t=m} \tilde{\tilde{E}}_{2}^{s, t, q}=\tilde{\tilde{E}}_{2}^{m, q}$ is associated to $\operatorname{Ext}_{A}^{m, q}\left(M, Z_{p}\right), \tilde{d}_{r}: \tilde{\tilde{E}}_{r}^{s, t, q} \rightarrow$ $\tilde{\tilde{E}}_{r}^{s+t, t+r-1, q+r-1}$, and the group $\sum_{t-q=l} \tilde{\tilde{E}}_{s, t, q}^{\infty}$ is associated to $\operatorname{Ext}_{A_{p}^{U}}^{s, 1}\left(U_{p}^{*}(Y), U_{p}^{*}(P)\right)$, $M=H^{*}\left(Y, Z_{p}\right)$.

We prove Theorem 12.1. In the Steenrod algebra $A$ for $p>2$ there is defined a second grading — the so-called "type in the sense of Cartan," equal to the number of occurrences of the homomorphism $\beta$ in the iteration. We shall denote by $\tau(a) \geq$ 0 the type of the operation $a \in A$, with $A=\sum_{\tau} A^{\tau}$, where $\tau$ is the type and $A^{\tau_{1}} \cdot A^{\tau_{2}} \subset A^{\tau_{1}+\tau_{2}}$. By the same token, for any $Y \in D_{p}$ there is an extra grading - the type $\tau$ - in the groups $\operatorname{Ext}_{A}\left(M, Z_{p}\right)$, and

$$
\operatorname{Ext}_{A}^{s, l}\left(M, Z_{p}\right)=\sum_{\tau \geq 0} \operatorname{Ext}_{A}^{s, l-\tau, \tau}\left(M, Z_{p}\right),
$$


where $l-\tau \equiv 0 \bmod 2 p-2$. We note that for $Q \subset A, \tau\left(Q_{r}\right)=1$ and $\tau\left(P^{k}\right)=0$. It is also obvious that $\tau\left(h_{i}\right)=1, h_{i} \in \operatorname{Ext}_{Q}^{1}\left(Z_{p}, Z_{z}\right)$, and the type is an invariant of the spectral sequence $\left(\tilde{d}_{r}, \tilde{E}_{r}\right)$ for $r \geq 1$.

Since the type is trivial on the ring $A / / Q$, and $A / / Q \subset A$, all $\tilde{d}_{r}=0$ for $r \geq 2$, since on the groups $\operatorname{Ext}_{A / / Q}^{s, q}\left(Z_{p}, \operatorname{Ext}_{Q}^{t}\left(Z_{p}, Z_{p}\right)\right)$ the type $\tau=t$ and $\tau\left(d_{r} y\right)=\tau(y)$ for $r \geq 1$.

This implies the isomorphism

$$
\operatorname{Ext}_{A}^{m, q}\left(M, Z_{p}\right)=\sum_{s+t=m} \operatorname{Ext}_{A / / Q}^{s, q}\left(M, \operatorname{Ext}_{Q}^{t}\left(Z_{p}, Z_{p}\right)\right)
$$

and $\tilde{E}_{2}=\tilde{E}_{\infty}$. The theorem is proved.

From the proof of Theorem 12.1 follows

Corollary 12.2. The second term of the "algebraic Adams spectral sequence" $\left(\tilde{\tilde{E}}_{r}, \tilde{\tilde{d}}_{r}\right)$ of Corollary 12.1 is canonically isomorphic to the sum $\sum \tilde{\tilde{E}}_{2}^{s, t, q}$, where $\tilde{\tilde{E}}_{2}^{s, t, q}=\operatorname{Ext}_{A}^{s, t, q}\left(M, Z_{p}\right), t$ is the Cartan type, $M=H^{*}\left(Y, Z_{p}\right)$ for $Y \in D_{p}$, and $\sum_{t+s=m} \operatorname{Ext}_{A}^{s, t, q}\left(M, Z_{p}\right)=\operatorname{Ext}_{A}^{m, q}\left(M, Z_{p}\right)$.

In this spectral sequence

$$
\tilde{\tilde{d}}_{r}: \tilde{\tilde{E}}_{r}^{s, t, q} \rightarrow \tilde{\tilde{E}}_{r}^{s+1, t+r-1, q+r-1}
$$

and the group $\sum_{t-q=l} \tilde{\tilde{E}}_{\infty}^{r, t, q}$ is associated to $\operatorname{Ext}_{A_{p}^{U}}^{s, l}\left(U_{p}^{*}(Y), U_{p}^{*}(P)\right)$.

From the geometric realization of double complexes as defined above, Theorem 12.1 and Corollaries 12.1, 12.2, there follows

Theorem 12.2. The "algebraic Adams spectral sequence" $\left(\tilde{\tilde{E}}_{r}, \tilde{\tilde{d}}_{r}\right)$ is associated to the Adams spectral sequence $\left(E_{r}, d_{r}\right)$ in $H^{*}\left(, Z_{p}\right)$-cohomology theory for all $p>2$ in the following sense:

1) $E_{2}^{m, q}=\sum_{s+t=m} \tilde{\tilde{E}}_{2}^{s, t, q}=\operatorname{Ext}_{A}^{m, q}\left(M, Z_{p}\right)$;

2) if for some $y \in E_{2}^{s, t, q}$ we have $\tilde{\tilde{d}}_{i}(y)=0$ for $i<k$ and $\tilde{\tilde{d}}_{k}(y) \neq 0$, then there is a $\tilde{y}$ such that $\phi(y-\tilde{y}) \geq \phi(\tilde{y})+1, d_{i}(\tilde{y})=0$ for $i<k$, and $d_{k}(\tilde{y}) \neq 0$, and moreover $\phi\left(d_{k} \tilde{y}\right)=\phi(\tilde{y})+1$, where $\phi(\tilde{y})=\phi(y)=t$ and $\phi\left(d_{k} \tilde{y}-d_{k} y\right)>\phi(\tilde{y})+1$;

3) if $\tilde{y} \in \operatorname{Ext}_{A}^{m, q}\left(M, Z_{p}\right)$ is such that $d_{i}(\tilde{y})=0$ for $i<k$ and $\phi\left(d_{k} \tilde{y}\right)>\phi(\tilde{y})+1$, then for the projection $y$ of the element $\tilde{y}$ in $\operatorname{Ext}_{A}^{m-\phi(\tilde{y}), \phi(\tilde{y}), q}\left(M, Z_{p}\right)$ we have the equation $\tilde{\tilde{d}}_{i}(y)=0$ for $i \leq k$ (we note that for elements $y \in$ $\left.\sum_{t \geq a} \operatorname{Ext}_{A}^{s, t, q}\left(M, Z_{p}\right), \varphi(y) \geq \alpha\right)$.

The groups $\operatorname{Ext}_{A^{U}}^{1, s}\left(U_{p}^{*}(P), U_{p}^{*}(P)\right)$ were computed in previous sections; they are cyclic for $s=2 k(p-1)$ of order $P^{f(k)}$, where $f(k)-1$ is the exponent of the greatest power of $p$ which divides $k$.

Corollary 12.3. The generator $\alpha_{k}$ of the group $\operatorname{Ext}_{A_{p}^{U}}^{1,2 k(p-1)}\left(\Lambda_{p}, \Lambda_{p}\right)$ has filtration $(1, k-f(k))$ or, in other words, $\phi\left(\alpha_{k}\right)=k-f(k)$ in the term $E_{\infty}$ of the "algebraic Adams spectral sequence" $\left(\tilde{\tilde{E}}_{r}, \tilde{\tilde{d}}_{r}\right)$ for $p>2$. Since $\operatorname{Ext}_{A_{p}^{U}}^{1, *}\left(\Lambda_{p}, \Lambda_{p}\right)$ consists of cycles for all Adams differentials in $U_{p}^{*}$-theory, $d_{i}\left(\alpha_{k}\right)=0, i \geq 2$, and there is an 
associated element $\tilde{\alpha}_{k} \in \pi_{*}\left(S^{n}\right)$; we have $\phi\left(\tilde{\alpha}_{k}\right)=k-f(k), i_{U}\left(\tilde{\alpha}_{k}\right)=1, i_{H}\left(\tilde{\alpha}_{k}\right) \leq$ $k-f(k)+1$.

Proof. As was shown in $\S 11$, the homomorphism $\operatorname{Ext}_{D_{1}}^{1}\left(\Lambda_{p, 1}, \Lambda_{p, 1}\right) \rightarrow$ $\operatorname{Ext}_{A_{p}^{U}}^{1}\left(\Lambda_{p}, \Lambda_{p}\right)$ is an epimorphism for $p>2$. For the ring $D_{1}$ and the module $\Lambda_{p, 1}=Q_{p}\left[x_{1}\right]$ the $\operatorname{ring} C=\operatorname{Hom}^{*}\left(F, \Lambda_{p, 1}\right)$ was determined (see Lemma 11.4), where $\phi(x)=1, \phi\left(h_{j}\right)=0, \phi(p)=1$ and $d\left(x^{k}\right) \sum\left(\begin{array}{l}k \\ j\end{array}\right) p^{j} x^{k-j} h_{j}$. The element $\alpha_{k}$ was represented by $\alpha_{k}=\left(1 / p^{f(k)}\right) d\left(x^{k}\right)$.

From this we have:

$$
\varphi\left(\alpha_{k}\right)=\min j\left[\varphi\left(\begin{array}{l}
k \\
j
\end{array}\right)+j+k-j-f(k)\right]=k-f(k)
$$

Thus, the filtration $\phi$ of the element $\alpha_{k}$ is equal to $k-f(k)$, since the filtration $\phi$ is induced by the filtration in the ring $\Lambda_{p}$. The Corollary is proved.

As is known, the groups $\operatorname{Ext}_{A}^{1, s}\left(Z_{p}, Z_{p}\right)$ are equal to $Z_{p}$ for $s=1$ or $s=2 p^{j}(p-1)$ and are generated by elements $u_{j}, j \geq 0$, of type 0 for $s=2 p^{j}(p-1)$ and $h_{0} \in$ $\operatorname{Ext}_{A}^{0,1,1}$ of type 1 in the sense of Cartan.

Hence, $u_{i} \in \operatorname{Ext}_{A}^{1,0,2 p^{i}(p-1)}\left(Z_{p}, Z_{p}\right)$ and $h_{0} \in \operatorname{Ext}_{A}^{0,1,1}\left(Z_{p}, Z_{p}\right)$, where $\operatorname{Ext}_{A}^{m, q}=$ $\sum_{s+t=m} \operatorname{Ext}_{A}^{s, t, q}$ and $t$ is the type. In the groups $\operatorname{Ext}_{A}^{2,2 p^{i}(p-1)}$ there are nonzero elements $y_{i}, i \geq 1$, having type 0 .

Corollary 12.4. In the "algebraic Adams spectral sequence" we have the equation $\tilde{\tilde{d}}_{2}\left(u_{i}\right)=h_{0} \gamma_{i}$, for $i \geq 1$.

The proof, by analogy with the proof of Corollary 12.3 , follows easily from the structure of the homomorphism $\beta$ in $H\left(C \otimes Z_{p}\right)$, where $\beta\left(h_{p} i\right)=\gamma_{i}$ for $i \geq 1$ (see Lemma 11.4).

Thus, we see that with the help of the "algebraic Adams spectral sequence" it is not only possible to prove the absence of elements with Hopf-Steenrod invariant 1, but also to compute (ordinary) Adams differentials by purely algebraic methods which come from the ring $A^{U}$.

Conjecture. For $p>2$ the "algebraic Adams spectral sequence," which converges to $\operatorname{Ext}_{A^{U}}\left(U^{*}(P), U^{*}(P)\right) \otimes_{Z} Q_{p}$, coincides with the "real" Adams spectral sequence, and the homotopy groups of spheres $\pi_{*}\left(S^{n}\right) \otimes_{Z} Q_{p}$ are associated to $\operatorname{Ext}_{A^{U}}\left(U^{*}(P), U^{*}(P)\right) \otimes_{Z} Q_{p}$. \{Equivalently: all differentials $d_{r}, r \geq 2$, are zero in the Adams spectral sequence over $\left.U_{p}^{*} \cdot\right\}$

We now consider $p=2$. As was indicated earlier, here there are two spectral sequences $\left(\tilde{E}_{r}, \tilde{d}_{r}\right)$ and $\left(\tilde{\tilde{E}}_{r}, \tilde{\tilde{d}}_{r}\right)$, where $\tilde{\tilde{E}}_{2}=\tilde{E}_{2}=\operatorname{Ext}_{A / / Q}\left(M, \bar{\Lambda}_{2}\right), M=H^{*}\left(X, Z_{2}\right)$, and $\bar{\Lambda}_{2}=\operatorname{Ext}_{Q}^{* *}\left(Z_{2}, Z_{2}\right)$ is associated to $U_{2}^{*}(P)=\Lambda_{2}$. The sequence $\left(\tilde{\tilde{E}}_{r}, \tilde{\tilde{d}}_{r}\right)$ converges to $\operatorname{Ext}_{A_{2}^{U}}\left(U_{2}^{*}(X), \Lambda_{2}\right)$ and $\left(\tilde{E}_{r}, \tilde{d}_{r}\right)$ converges to $\operatorname{Ext}_{A}\left(M, Z_{2}\right)$.

By analogy with Theorem 12.2 for $p>2$, here we have

Theorem 12.3. The differentials $\tilde{d}_{r}$ are associated with the Adams differentials in Cobordism theory on the group $\tilde{\tilde{E}}_{\infty}$ associated with $\operatorname{Ext}_{A_{2}^{U}}\left(U_{2}^{*}(X), \Lambda_{2}\right)$. The differentials $\tilde{\tilde{d}}_{r}$ are associated with the Adams differentials in $H^{*}\left(, Z_{2}\right)$-theory on the groups $\tilde{E}_{\infty}$ associated with $\operatorname{Ext}_{A}\left(H^{*}\left(X, Z_{2}\right), Z_{2}\right)$, where $X \in D$. 
The proof of Theorem 12.3, as of 12.2 , follows immediately from the properties of the geometric realization of the double complex.

Thus, for $p=2$, it is possible to compute the Adams differentials in $H^{*}\left(, Z_{2}\right)$ theory, starting from cobordism, and conversely.

Question. Do the algebraic Adams spectral sequences $\tilde{E}_{r}$ and $\tilde{\tilde{E}}_{r}$ define the real Adams spectral sequences in both theories?

In any case in all examples known to the author all Adams differentials are subsumed under this scheme.

Example. Let $X=M S U$. We consider $\operatorname{Ext}_{A / / Q}(M, \bar{\Lambda})$, where $M=$ $H^{*}\left(X, Z_{2}\right)$. We write an $A / / Q$-resolution of the module $M$ :

$$
\left(\cdots \rightarrow C_{i} \stackrel{d}{\rightarrow} C_{i-1} \rightarrow \cdots \rightarrow C_{0} \stackrel{\varepsilon}{\rightarrow} M\right)=C .
$$

We recall that $M=F+\sum_{\omega} M_{\omega}$, where $F$ is $A / / Q$-free and $M \omega$ has one generator $u_{\omega}$ for all $\omega=\left(4 k_{1}, \ldots, 4 k_{s}\right)$ and is given by the relations $\mathrm{Sq}^{2} u_{\omega}=0$ over $A / / Q$, where $\operatorname{dim} u_{\omega}=8 \sum k_{j}$. Hence one can assume that $C=C(F)+\sum C\left(M_{\omega}\right)$, where $C(F)=F$ and $C\left(M_{\omega}\right)$ has the form:

$$
C\left(M_{\omega}\right)=\left(\rightarrow \ldots \stackrel{d}{\rightarrow} A / / Q \stackrel{d}{\rightarrow} A / / Q \rightarrow \ldots \stackrel{d}{\rightarrow} A / / Q \stackrel{\varepsilon}{\rightarrow} M_{\omega}\right)
$$

where $u_{i}$ is a generator of $C_{i}\left(M_{\omega}\right)$ and $d u_{i}=\mathrm{Sq}^{2} u_{i-1}$. The action of $\mathrm{Sq}^{2}$ on $\bar{\Lambda}_{2}$ was indicated earlier: $\bar{\Lambda}_{2}=Z_{2}\left[h_{0}, \ldots, h_{i}, \ldots\right], \operatorname{dim} h_{i}=\left(1,2^{i+1}-1\right), i \geq 0$, while $\mathrm{Sq}^{2} h_{1}=h_{0}$.

There follows straightforwardly (by direct calculation)

Lemma 12.5. $\operatorname{Ext}_{A / / Q}^{* * *}\left(M_{\omega}, \bar{\Lambda}_{2}\right)$ for $\omega=(0)$ has a system of multiplicative generators:

$$
h_{0} \in \mathrm{Ext}^{0,1,1}, \quad x_{1} \in \mathrm{Ext}^{1,0,2}, \quad h_{i} \in \mathrm{Ext}^{0,1,2^{i+1}-1}, \quad i \geq 2, \quad y \in \mathrm{Ext}^{0,2,6}
$$

and is given by the relation $h_{0} x_{1}=0$.

We note that the dimension of $\operatorname{Ext}^{s, t, q}$ in $H^{*}\left(, Z_{2}\right)$ is equal to $(s+t, q)$ and the dimension in $U_{2}^{*}$-theory is equal to $(s, q-t)$ (see above).

We now describe the spectral sequences $\tilde{E}_{r} \searrow \operatorname{Ext}_{A}$ and $\tilde{\tilde{E}}_{r} \searrow \operatorname{Ext}_{A_{2}^{U}}$.

Lemma 12.6. a) The spectral sequence $\left(\tilde{E}_{r}, \tilde{d}_{r}\right)$ is such that:

$$
\begin{gathered}
\tilde{d}_{3}(y)=x_{1}^{3}, \quad \tilde{d}_{3}\left(h_{0}\right)=\tilde{d}_{3}\left(x_{1}\right)=\tilde{d}_{3}\left(h_{i}\right)=\tilde{d}_{3}\left(v_{\omega}=0\right), \\
\tilde{d}_{3} \mid \operatorname{Hom}_{A / Q}\left(F, \bar{\Lambda}_{2}\right)=0
\end{gathered}
$$

and all $\tilde{d}_{r}=0$ for $r=3$.

b) The spectral sequence $\left(\tilde{\tilde{E}}_{r}, \tilde{\tilde{d}}_{r}\right)$ is such that:

$$
\begin{gathered}
\tilde{\tilde{d}}_{2}\left(v_{\left(2^{i}\right)}\right)=x_{1} h_{i+2}, \quad i \geq 0, \\
\tilde{\tilde{d}}_{2}\left(x_{1}\right)=\tilde{\tilde{d}}_{2}\left(h_{i}\right)=\tilde{\tilde{d}}_{2}(y)=\tilde{\tilde{d}}_{2}\left(v_{(k)}\right)=0, \quad k \neq 2^{i}, \\
\tilde{\tilde{d}}_{2}\left(\operatorname{Hom}_{A / / Q}\left(F, \Lambda_{2}\right)\right)=0, \\
M=F+\sum_{\omega} M_{\omega},
\end{gathered}
$$


S. P. NOVIKOV

where $F$ is $A / / Q$-free, $\tilde{\tilde{d}}_{r}=0$ for $r \leq 2$ (we note that $v_{\omega}$ is conjugate to the generator $u_{\omega}$ of the module $M_{\omega}, \omega=\left(k_{1}, \ldots, k_{s}\right), \operatorname{dim} u_{\omega}=8\left(\sum k_{j}\right)$ and $v_{\omega} v_{\omega_{1}}=$ $v_{\left(\omega, \omega_{1}\right)}$ by virtue of the diagonal in the module $\left.M\right)$.

The proof of Lemma 12.6 for $\tilde{E}_{r}$ follows easily from the calculations [18] for $\operatorname{Ext}_{A}\left(M, Z_{2}\right)$. For the case $\left(\tilde{\tilde{E}}_{r}, \tilde{\tilde{d}}_{r}\right)$, part (b) of Lemma 12.6 follows from the fact that the elements $x, h_{i+2}$ must be zero in $\operatorname{Ext}_{A_{2}^{U}}\left(U_{2}^{*}\left(U_{2}^{*}(M S U), \Lambda_{2}\right)\right.$ on the basis of $\S 7$.

Corollary 12.5. For $M S U$, the Adams spectral sequence (in U-cobordlsm and $H^{*}\left(, Z_{2}\right)$-theory) determined by the algebraic spectral sequences $\tilde{E}_{r}$ and $\tilde{\tilde{E}}_{r}$.

In analogous fashion it can be shown that all known Adams differentials for $X=P$ in both homology theories (the case of the homotopy groups of spheres) are also determined by $\tilde{E}_{r}, \tilde{\tilde{E}}_{r}$ and $\tilde{d}_{r}, \tilde{\tilde{d}}_{r}$.

By analogy with the case $p>2$, bounds can be determined here also for the filtrations of elements $\operatorname{Ext}_{A^{U}}^{1}$ (see Corollary 12.3).

\section{Appendix 1. On the formal Group of "GEOMETRIC" COBORDism} (TheOrEM of A. S. MišČEnKo)

We consider an arbitrary complex $X$, the group $U^{*}(X)$ and its subgroup $\operatorname{Map}\left(X, M U_{1}\right) \subset U^{2}(X)$. In what follows we shall denote $\operatorname{Map}\left(X, M U_{1}\right)$ by $V(X)$. Since $M U_{1}=C P^{\infty}$ is an $H$-space, $V(X)$ becomes a group, which is communicative, and with respect to this law of multiplication we obviously have:

$$
V(X) \approx H^{2}(X, Z)
$$

How is this multiplication in $V(X)$ connected with operations in $U^{*}(X) \supset V(X)$ ?

As was already indicated in $\S 5$, we have

Lemma 1. a) If $u, v \in V(X)$ and $\oplus$ is the product in $V(X)$, then the law of multiplication $u \oplus v=f(u, v)$ has the form

$$
u \otimes v=u+v+\sum_{\substack{i \geq 1 \\ j \geq 1}} x_{i, j} u^{i} v^{i},
$$

where $x_{i j} \in \Lambda^{-1(i+j-1)}=\Omega_{U}^{2(i+j-1)}$ are coefficients independent of $u, v$,

b) $u \oplus v=v \oplus u$,

c) $(u \oplus v) \oplus w=u \oplus(v \oplus w)$,

d) there exists an inverse element $\bar{u}$, where $\bar{u} \oplus u=0$.

The proof of this lemma follows in an obvious way from the fact that $V(X) \approx$ $H^{2}(X, Z)$ and the possibility of computing all the coefficients on the universal example $X=C P^{\infty}$. We note that $x_{1,1}=\left[C P^{1}\right]$.

Thus, we have a commutative formal group with graded ring of coefficients $\Lambda$, and $\operatorname{dim} u=\operatorname{dim} v=2$. As is known, the structure of such a group is completely determined by a change of variables $g$ over the ring $\Lambda \otimes_{Z} Q, u \rightarrow g(u)=\sum_{i \geq 0} y_{i} u^{i+1}$, $y_{0}=1$, such that

We have the following

$$
g(u \oplus u)=g(u)+g(v)
$$


Theorem (A. S. Miščenko). The change of variables $u \rightarrow g(u)$, where $g(u)=$ $\sum_{n \geq 0} \frac{x_{n}}{n+1} u^{n+1}, x_{n}=\left[C P^{n}\right] \in \Lambda^{-2 n}$, reduces the formal group $V \otimes_{Z} Q$ to linear form $g(u \oplus v)=g(u)+g(v)$. Hence, the change $u \rightarrow g(u)$ reduces to linear form the formal group $V(X) \otimes Q$ for all $X$ and uniquely determines the structure of the one-dimensional formal group $V$ over the ring $\Lambda$.

Proof. We consider the ring $U^{*}\left(C P^{\infty}\right)=\Lambda[[u]]$ and the multiplication $C P^{\infty} \times$ $C P^{\infty} \rightarrow C P^{\infty}$, sending the one-dimensional canonical $U_{1}$-bundle $\xi$ over $C P^{\infty}$ into $\xi_{1} \otimes \xi_{2}$, where $\xi_{1}, \xi_{2}$ are canonical bundles over $C P \times C P$. This multiplication induces a diagonal $\Delta: U^{*}\left(C P^{\infty}\right) \rightarrow U^{*}\left(C P^{\infty}\right) \otimes_{\Lambda} U^{*}\left(C P^{\infty}\right)$, which gives the multiplication in $V\left(C P^{\infty}\right)$.

Let $u^{\prime}=g(u) \sum \lambda_{i} u^{i}$, where $\Delta\left(u^{\prime}\right)=u^{\prime} \otimes 1+1 \otimes u^{\prime}$. Then $g$ is the desired change of variables.

We compute the coefficients $\lambda_{i}$. Let $S_{(k)} \in A^{U}$ (see $\S 5$ ).

We have the easy

Lemma 2. The operations $S_{(k)}$ form a system of multiplicative generators for the ring $S \otimes Q$. If $\sigma_{k}^{*}(x)=0$ for all $k, x \in \Lambda$, then $x=0$.

Proof. We order the partitions $\omega$ naturally (by length) and consider

$$
\begin{gathered}
S_{(k)} S_{\omega}\left(u_{1} \ldots u_{n}\right)=S_{(k)} \sum u_{i}^{k_{1}+1} \ldots u_{s}^{k_{s}+1} u_{s+1} \circ \ldots \circ u_{n}, \\
\omega=\left(k_{1}, \ldots, k_{s}\right), \\
S_{(k)} S_{\omega}\left(u_{1} \ldots u_{n}\right)=\sum a_{i} S_{\omega_{i}}\left(u_{1} \ldots u_{n}\right)+a_{0} S_{(k, \omega)}\left(u_{1} \ldots u_{n}\right),
\end{gathered}
$$

where $a_{0} \neq 0, \omega_{i}=\left(k_{1}, \ldots, k_{i}+k, k_{i+1}, \ldots, k_{s}\right)$. Since by the induction hypothesis all $S_{\omega_{i}}$ can be expressed by the $S_{\left(k_{j}\right)}$, the same is true for $S_{(\omega, k)}$. Since all $S_{\omega}$ can be expressed by the $S_{(k)}$, the lemma is proved.

We note the following equation:

$$
\begin{aligned}
S_{(k)} u^{i}=\sum_{i} S_{(k)}\left(\lambda_{i} u^{i}\right)=\sum_{i}\left(\sigma_{k}^{*}\left(\lambda_{i}\right) u^{i}+i \lambda_{i} u^{i+k}\right) & \\
& =\sum_{i}\left(\sigma_{(k)}^{*}\left(\lambda_{i}\right)+(i-k) \lambda_{i-k}\right) u^{i}
\end{aligned}
$$

We set

$$
u^{\prime k}=\sum_{i} \lambda_{i}^{(k)} u^{i}, \quad u^{k}=\sum_{i} \mu_{i}^{(k)} u^{\prime i}, \quad \sum_{i} \lambda_{i}^{(k)} \mu_{j}^{(i)}=\delta_{j}^{k}
$$

Obviously, $S_{(k)} \Delta u^{\prime}=\Delta S_{(k)} u^{\prime}$, since $\Delta u^{\prime}=u^{\prime} \otimes 1+1 \otimes u^{\prime}$. Since

$$
S_{(k)} u^{\prime}=\sum_{i}\left(\sigma_{(k)}^{*}\left(\lambda_{i}\right)+(i-k) \lambda_{i-k}\right) u^{i}=\sum_{i} \sum_{j}\left(\sigma_{(k)}^{*}\left(\lambda_{i}\right)+(i-k) \lambda_{i-k}\right) \mu_{j}^{(i)} u^{\prime j}
$$


we have

$$
\begin{gathered}
\Delta S_{(k)} u^{\prime}=S_{(k)} \Delta u^{\prime}=\sum_{i} \sum_{j}\left(\sigma_{(k)}^{*}\left(\lambda_{i}\right)+(i-k) \lambda_{i-k}\right) \mu_{j}^{(i)}\left(u^{\prime} \otimes 1+1 \otimes u^{\prime}\right)^{j} \\
=\sum_{j} \sum_{i}\left[\left(\sigma_{(k)}^{*}\left(\lambda_{i}\right)+(i-k) \lambda_{i-k}\right) \mu_{j}^{(i)}\left(\sum_{\alpha+\beta=j}\left(\begin{array}{c}
\alpha+\beta \\
\alpha
\end{array}\right) u^{\prime \alpha} \otimes u^{\prime \beta}\right)\right] \\
=S_{(k)}\left(u^{\prime} \otimes 1+1 \otimes u^{\prime}\right) .
\end{gathered}
$$

It obviously follows that for $\alpha \neq 0, \beta \neq 0$ we have:

$$
\sum_{i}\left(\sigma_{(k)}^{*} \lambda_{i}+(i-k) \lambda_{i-k}\right) \mu_{j}^{(i)}=0, \quad j=\alpha+\beta \geq 2 .
$$

Since $\mu_{1}^{(i)}=0$ for all $i \geq 2, \mu_{1}^{(i)}=1, \lambda_{1}=1$ and $\sigma_{(k)}^{*}+(i-k) \lambda_{i-k}=0, k \geq 1$, we have

$$
\sum_{i}\left(\sigma_{(k)}^{*} \lambda_{i}+(i-k) \lambda_{i-k}\right) \mu_{j}^{(i)}=0
$$

for all $j \geq 1$, and since $\sum_{j} \mu_{j}^{(i)} \lambda_{s}^{(j)}=\delta_{j}^{i}$, we have

$$
\sum_{j} \sum_{i}\left(\sigma_{(k)}^{*} \lambda_{i}+(i-k) \lambda_{i-k}\right) \mu_{j}^{(i)} \lambda_{s}^{(j)}=\sigma_{(k)}^{*} \lambda_{i}+(i-k) \lambda_{i-k}=0 .
$$

Hence,

$$
\sigma_{(k)}^{*} \lambda_{i}=-(i-k) \lambda_{i-k}
$$

Further, since $\sigma_{(k)}^{*}\left[C P^{n}\right]=-(n+1)\left[C P^{n-k}\right](\operatorname{see} \S 5$, Lemma 5), it follows that $\bar{\lambda}_{i}=x_{i-1} / i, x_{j}=\left[C P^{j}\right] \in \Lambda^{-2 j}$ satisfies the condition $\sigma_{(k)}^{*} \bar{\lambda}_{i}=-(i-k) \bar{\lambda}_{i-k}$ for all $i, k$. By Lemma $2, \bar{\lambda}_{i}=\lambda_{i}$, and the theorem is proved.

Remark. For a quasicomplex manifold $X$, the group $V(X)$ is isomorphic to $H_{2 n-2}(X)$ and the meaning of the sum $u \oplus v$ is such that the homology class $\nu(u) \nu(v)$ is realized by the inclusion of the submanifold $V_{1} \otimes V_{2}$, where $u \in U_{2 n-2}(X), v \in U_{2 n-2}$ are realized by the submanifolds $V_{1}, V_{2} \subset X$. Then the series

$$
u \oplus v=u+v+\cdots=f(u, v)
$$

must be considered in the intersection $\operatorname{ring} U_{*}(X)$.

\section{Appendix 2. On analogues of the AdAms operations in $U^{*}$-THEORY}

Analogues of the Adams operations $\Psi_{U}^{k} \in A^{U} \otimes_{Z} Z[(1 / k)]$ were defined in $\S 5$ in the following way:

a) $\Psi_{U}^{k}(x y)=\Psi_{U}^{k}(x) \Psi_{U}^{k}(\gamma)$

b) $k \Psi_{U}^{k}(x)=x \oplus \cdots \oplus x$ ( $k$ times), where $x \in V(X)$.

Thus, the series $\Psi_{U}^{k}$ has the form:

$$
k \Psi_{U}^{k}(x)=g^{-1}(k g(x))=f(x, f(x, \ldots, f(x, x), \ldots),
$$

where $f(u, v)$ is the law of addition in the formal group $V(X)$ and

$$
g(x)=\sum_{k \geq 0} \frac{x_{k}}{k+1} x^{k+1}, \quad x_{j}=\left[C P^{j}\right], \quad x \in V(X),
$$

the basis of Appendix $1, g^{-1}(g(x))=x$. 
From the associativity of the law of multiplication in $V(X)$ follows the equation:

$$
\Psi_{U}^{k}\left(\Psi_{U}^{l}(x)\right)=\Psi_{U}^{k l}(x) .
$$

Hence, always $\Psi_{U}^{k} \circ \Psi_{U}^{l}=\Psi_{U}^{k l}$ in $A^{U} \otimes_{Z} Q$, since for any $n \rightarrow \infty$ and $u=u_{1} \ldots u_{n}$ we have $\Psi_{U}^{k} \Psi_{U}^{k}(u)=\Psi_{U}^{k l}$ by virtue of properties (a) and (b).

Of the assertions in Lemma 5.8, only part (d) is nontrivial, and it asserts that $\Psi_{U}^{k, *}(y)=k^{i} y, y \in \Lambda^{-2 i}=\Omega_{U}^{2 i}$.

Theorem 1. ${ }^{5}$ If $a \in A^{U}$ is an arbitrary cohomology operation of dimension $2 m$, then we have the following commutation law:

$$
a \Psi_{U}^{k}=k^{m} \Psi_{U}^{k} \circ a
$$

Proof. Let $a_{m}=S(m) \in A^{U}$ and $u \in V\left(C P^{\infty}\right) \subset U^{2}\left(C P^{\infty}\right)$. Then

$$
\begin{gathered}
a_{m}(u)=u^{m+1} \\
\Psi_{U}^{k}\left(a_{m} u\right)=\Psi_{U}^{k}\left(u^{m+1}\right)=\Psi_{U}^{k}(u)^{m+1}=\frac{1}{k^{m+1}}(u \oplus \cdots \oplus u)^{m+1} \\
=\frac{1}{k^{m}} a_{m}\left(\frac{u \oplus \cdots \oplus u}{k}\right)=\frac{1}{k^{m}} a_{m} \Psi_{U}^{k}(u),
\end{gathered}
$$

since $u \oplus \cdots \oplus u \in V\left(C P^{\infty}\right)$. Hence, for the operations $a_{(m)}=S_{(m)}$ the theorem is proved. From this Theorem 1 follows for all operations $S_{(\omega)}$, since by Lemma 2 of Appendix 1 the ring $S \otimes_{Z} Q$ is generated by the operations $S_{(k)}$.

Now let $a \in \Lambda^{-2 m}=U^{-2 m}(P)$. We assume by induction that for all operations in $\Lambda^{-2 j}, j<m$, the theorem is proved. This means that for $b \in \Lambda^{-2 j}, j<m$, we have:

$$
\Psi_{U}^{k, *}(b)=k^{j} b .
$$

In view of the fact that $\Psi_{U}^{k, *}\left(b_{1} b_{2}\right)=\Psi_{U}^{k, *}\left(b_{1}\right) \Psi_{U}^{k, *}\left(b_{2}\right)$, the theorem is also proved for all decomposable elements of $\Lambda^{-2 m}$. Let $a \in \Lambda^{-2 m}$ be an indecomposable element. We consider $\Psi_{U}^{k, *} \sigma_{\omega}^{*}(a)=k^{m-\operatorname{dim} \omega} \sigma_{\omega}^{*}(a)$ by induction, for $\omega \neq(0)$. Since

$$
\Psi_{U}^{k} S_{\omega}=k^{-\operatorname{dim} \omega} S_{\omega} \Psi_{U}^{k}
$$

we have

$$
\Psi_{U}^{k, *} \sigma_{\omega}^{*}(a)=\sigma_{\omega}^{*}\left(k^{m} a\right) .
$$

Hence, $\Psi_{U}^{k, *}(a)=k^{m} a$, since $\bigcap_{\omega>0} \operatorname{Ker} \sigma_{\omega}^{*}=0$.

Since Theorem 1 is proved for $\Lambda$ and $S$, it is also proved for $A^{U}=(\Lambda S)^{\wedge}$.

Thus, all assertions of Lemma 5.8 are proved.

We now consider an arbitrary ring $K$, the group of units $U_{k} \subset K$ and $A^{U} \otimes_{Z} K$. We define the following semigroups in $A^{U} \otimes_{Z} K$ :

1. The semigroup of multiplicative operations $a \in A^{U} \otimes_{Z} K$, where $\Delta a=a \otimes a \in$ $A^{U} \otimes_{\Lambda} A^{U} \otimes_{Z} K$.

2. The semigroup of multiplicative operations of dimension 0 ,

$$
A_{K}^{0} \subset A_{K} \subset A^{U} \otimes_{Z} K .
$$

3. The center $Z_{K} \subset A_{K}^{0}$ of the semigroup $A_{K}$.

\footnotetext{
${ }^{5}$ From Theorem 1 it follows easily that all operations $\Psi_{U}^{k}$ are well-defined over the integers on $U^{0}(X)$, as in $K$-theory.
} 
4. The "Adams operations" $\Psi_{U}^{q} \in A_{K}^{0}$, where $q \in U_{k}$ (the group of units), defined by the requirements of Theorem 1:

$$
\begin{aligned}
\Psi_{U}^{q}(a)= & q^{-m} a \Psi_{U}^{q}, \quad \operatorname{dim} a=2 m, \\
& \Psi_{U}^{q_{1}} \Psi_{U}^{q_{2}}=\Psi_{U}^{q_{1} q_{2}} .
\end{aligned}
$$

Just as earlier, a multiplicative operation $a \in A_{K}$ is defined by a series $a(u), u \in$ $U^{2}\left(C P^{\infty}\right) \otimes_{Z} K$ the canonical element, $a(u) \in \Lambda_{K}[[u]], \Lambda_{K}=\Lambda \otimes_{Z} K$.

We now consider the question of defining the Adams operation. Let $K=Q[t]$, $\Lambda_{K}=\Lambda \otimes_{Z} K$. We consider for all integral values $t$ the series $t \Psi_{U}^{t}(u) \in U^{*}\left(C P^{\infty}\right)$, defining the series $t \Psi_{U}(u) \in U^{*}\left(C P^{\infty}\right) \otimes_{Z} K$.

Remark. If $K$ is an algebra over $Q$, then the Adams operations $\Psi_{U}^{\alpha} \in A^{U} \otimes_{Z} K$ are always defined, since the series $t \Psi_{U}^{t}$ is divisible by $t$ and $\Psi_{U}^{t}(u) \in U^{*}(C P) \otimes_{Z} K$.

We have the following

Theorem 2. a) For any algebra $K$ over $Q$ without zero divisors and for $K=Q_{p}, Z$, the "Adams operations" $\Psi_{U}^{\alpha} \in A^{U} \otimes_{Z} K$ are defined, where $\alpha \in K^{*}$ in the $Q$-algebra case and $\alpha \in U_{p}$ in the case $K=Q_{p}$ \{i.e., $\left.U_{p}=U_{Q_{p}}\right\}, \alpha= \pm 1$ in the case $K=Z$, such that:

1) $\Psi_{U}^{\alpha_{1}} \Psi_{U}^{\alpha_{2}}=\Psi_{U}^{\alpha_{1} \alpha_{2}}$

2) $\Psi_{U}^{\alpha, *}: \Lambda_{K}^{-2 i} \rightarrow \Lambda_{K}^{-2 i}$ is multiplication by $\alpha^{i}$.

3) $\Psi_{U}^{\alpha} \circ a=\alpha^{-i} a \Psi_{U}^{\alpha}$, where $a \in A^{U} \otimes_{Z} K$ is of dimension $2 i$.

4) The series $\alpha \Psi_{U}^{\alpha}(u)$ for $u \in V\left(C P^{\infty}\right)$ makes the operation of raising to the power $\alpha, \alpha \in K^{*}$, well-defined in the formal group $V$.

b) The collection of all Adams operations forms a semigroup $K^{*} \approx \Psi(K)$ for a $Q$-algebra $K, \Psi(K) \approx U_{p}$ for $K=Q_{p}, \Psi(Z)=Z_{2}$, which coincides precisely with the center $Z_{K}$ of the semigroup $A_{K}^{0}$ of multiplicative operations of dimension 0 in the ring $A_{U} \otimes K$ for $K=Q_{p}, Z$, while for a $Q$-algebra $K$ the center consists of $\Psi(K)$ and the operator $\Phi$, where $\Phi(u)=g(u)$.

Remark. Although $a \in A_{K}$ is such that $\Delta a=a \otimes a$ and is given by a formal series beginning with 1 , where $a(u)=u+\ldots$, still the coefficients of the series lie in $\Lambda$ or $\Lambda \otimes K$, while the law of super-position of series $a_{1} \cdot a_{2}(u)$ takes into account the representation of $A^{U} \otimes K$ on $\Lambda \otimes K$. Hence $A_{K}$ is not a group (as usual in formal series of this kind), but a semigroup. An example of a "noninvertible" element $a \in A^{0}+K$ is given by the series

$$
\Phi(u)=\sum \frac{\left[C P^{i}\right]}{i+1} u^{i+1}=g(u),
$$

where $\Phi^{2}=\Phi$ and $\Phi^{*}(y)=0, y \in \Lambda^{2 j}$ for $j>0$.

We prove Theorem 2. Part (a) was essentially already proved above. In order to establish that $\Psi(K)=Z_{K}$, we consider an arbitrary element $a \in Z_{K}$ and we shall show that $a \in \Psi(K)$. Since the series $a(u)=u+\ldots$, we have $a^{*} \mid \Lambda^{0}=1$ and $a^{*} \mid \Lambda^{2}$ is multiplication by a number $\alpha \in K$. If $a^{*} \mid \Lambda^{-2 j}=0$ for all $j>0$, then it follows that $a^{*}=\Phi^{*}$ and hence $a=\Phi$, while $\Phi \notin A^{U} \otimes_{Z} Q_{p}$. It will be assumed that for some $j, a^{*} \mid \Lambda^{-2 j} \neq 0, j>0$. If $a^{*} \mid \Lambda^{-2 j}$ is the operator of multiplication by a number $k_{j}$, then it is easy to see that $k_{j}=k_{1}^{j}$ and $a=\Psi_{U}^{k_{1}}$, where $k_{1} \in K^{*}$ or $k_{1} \in U_{p}$. We shall show that for all $j$ the operator $a^{*}$ is multiplication by a number $k_{j}$. If $j_{0}$ is the first number for which $a^{*} \mid \Lambda^{-2 j_{0}}$ is not multiplication by 
a number, then, nevertheless, on the decomposable elements $\bar{\Lambda}^{-2 j_{0}} \subset \Lambda^{-2 j_{0}}, a^{*}$ is multiplication by a number in view of the fact that $a^{*}(x y)=a^{*}(x) a^{*}(y)$. If $y \in \Lambda^{-2 j_{0}}$ is an indecomposable element then $a^{*}(y)=\lambda y+\bar{y}, \bar{y} \subset \tilde{\Lambda}$ and $\bar{y}=0$. Let $b \in A_{K}^{0}$ be such that $b^{*}(y)=\mu y+\overline{\bar{y}}$, where $\overline{\bar{y}} \in \Lambda, \overline{\bar{y}} \neq 0$. Then $b^{*} a^{*} \neq a^{*} b^{*}$ on $\Lambda^{-2 j_{0}}$, which is impossible. The theorem is proved.

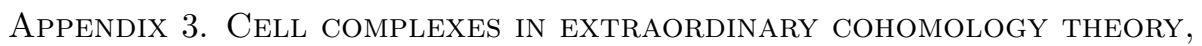 $U$-COBORDISM AND $k$-THEORY}

Let $X$ be a homology theory with a multiplicative stable spectrum, $X \otimes X^{*} \rightarrow X$, and let $\Lambda^{*}=X^{*}(P)$ be the cohomology ring of a point. We require that $\Lambda^{*}$ be a ring with identity. We note that $\Lambda=X_{*}(P)$ is also a ring, and we have the formulas $\Lambda=\operatorname{Hom}_{\Lambda^{*}}^{*}\left(\Lambda^{*}, \Lambda^{*}\right)$ and $\Lambda^{*}=\operatorname{Hom}_{\Lambda}^{*}(\Lambda, \Lambda)$. Obviously, the rings $\Lambda$ and $\Lambda^{*}$ are isomorphic and $\Lambda^{i}=\Lambda^{*-i}, \Lambda^{i}=0, i<0$.

Let $K$ be a cell complex and $K^{i} \subset K$ be its skeleton of dimension $i$. We construct a "cell complex of $\Lambda$-modules" $S_{X}(K)$ :

a) if $\operatorname{dim} K=0$, then $S_{X}(K)$ is a free complex $\sum_{P} \Lambda(P)$, where the $P$ are the vertices of $K$ and $\Lambda(P)$ is a one-dimensional free module with generator $u_{P}$ : we set $\partial u_{P}=0$.

b) Suppose that for all $K^{j}, j<i, S_{X}\left(K^{j}\right)$ has been constructed so that $\partial \lambda=\lambda \partial$, $\lambda \in \Lambda$, and the generators of $S_{X}\left(K^{j}\right)$ are in one-one correspondence with the cells of $K^{j}$

We consider the pair $\left(K^{j}, K^{i-1}\right)$, where $K^{i} / K^{i-1}$ is a bouquet of spheres $S_{1}^{i} \vee$ $\cdots \vee S_{q_{i}}^{i}$. We adjoin to $S_{X}\left(K^{i-1}\right)$ free generators $u_{1}, \ldots, u_{q_{i}}$ of dimension $i$. A differential in the complex $S_{X}\left(K^{i-1}\right)+\Lambda\left(u_{1}\right)+\cdots+\Lambda\left(u_{q_{i}}\right)$ is introduced as follows:

1) $\partial \lambda=\lambda \delta, \lambda \in \Lambda$;

2) $\partial u_{j}=z_{j} \in S_{X}\left(K^{i-1}\right)$, where $z_{j}$ is such that $\partial z_{j}=0$ in $S_{X}\left(K^{i-1}\right)$ and the homology class $\left[z_{j}\right] \in X_{*}\left(K^{i-1}\right)$ is represented by the element equal to $\partial u_{j}$, where $\partial: X_{*}\left(S_{1}^{i} \vee \cdots \vee S_{q_{i}}^{i}\right) \rightarrow X_{*}\left(K^{i-1}\right)$ is the boundary homomorphism of the pair $\left(K^{i}, K^{i-1}\right)$ and $u_{j} \in X_{*}\left(K^{i} / K^{i-1}\right)$ corresponds to the sphere $S_{j}^{i}$.

Thus, a complex $S_{X}(K)$ of free modules arises.

Lemma 1. The complex $S_{X}(K)$ is uniquely defined up to the choice of the system of generators, and the differential $\partial$ in $S_{X}$ coincides up to higher filtration with the homology one. Obviously, $H\left(S_{X}(K), \partial\right)=X_{*}(K)$ as $\Lambda$-modules.

For a cellular map $Y_{1} \rightarrow Y_{2}$, there is defined analogously a morphism of free complexes $S_{X}\left(Y_{2}\right) \rightarrow S_{X}\left(Y_{2}\right)$, also unique.

Let $Y=Y_{1} \times Y_{2}$ with the natural cellular subdivision.

Question. When is there defined a pairing

$$
S_{X}\left(Y_{1}\right) \otimes_{\Lambda} S_{X}\left(Y_{2}\right) \rightarrow S_{X}\left(Y_{1} \times Y_{2}\right),
$$

which is an isomorphism of complexes?

Now let $X=U$.

Conjecture. For a pair $Y_{1}, Y_{2}$, the complex $S_{U}\left(Y_{1} \times Y_{2}\right)$ is homo topic ally equivalent to the tensor product

$$
S_{U}\left(Y_{1}\right) \otimes_{\Omega_{U}} S_{U}\left(Y_{2}\right)
$$


Let $A$ be an arbitrary $\Omega_{U}$-module. The homology of the complex $S_{U} \otimes \Omega_{U} A$ we shall denote by $U_{*}(Y, A)$, and the homology of the complex $\operatorname{Hom}_{\Omega_{U}}^{*}\left(S_{U}, A\right)$ by $U^{*}(Y, A)$ (cohomology with coefficients in $\left.A\right)$.

We shall indicate important examples:

1. $A=\Omega_{U}$ is a one-dimensional free module.

2. $A=Z[x], \operatorname{dim} x=2$, and the action of $\Omega_{U}$ on $A$ is such that $y\left(x^{m}\right)=$ $T(y) x^{m+i}$, where $y \in \Omega_{U}, 2 i=\operatorname{dim} y$, and $T(y)$ is the Todd genus. Here $A$ is a ring and there is defined a homomorphism $\lambda: \Omega_{U} \rightarrow Z[x]$, such that $\lambda(y)=T(y) x^{i}$.

3. $A=Z\left[x, x^{-1}\right]$, where $\operatorname{dim} x=2, \operatorname{dim} x^{-1}=-2$ and $x x^{-1}=1$. Here $A$ is a ring, while $\Omega_{U}$ acts on $A$ just as in example 2: $y\left(x^{m}\right)=T(y) x^{m+i},-\infty<m<\infty$.

4. $A=Z$, where $Z=\Omega_{U} / \Omega_{U}^{+}, \Omega_{U}^{+}$is the kernel of the augmentation $\Omega_{U} \rightarrow Z$ and the action of $\Omega_{U}$ on $Z$ is the natural one.

Conjecture. For the $\Omega_{U}$-modules $A=\Omega_{U}, Z[x], Z\left[x, x_{-1}\right], Z$, the corresponding cohomology groups $U^{*}(, A)$ are isomorphic, respectively, to the cobordism theory $U^{*}$ for $A=\Omega_{U}$, to stable $k^{*}$-theory for $A=Z[x]$, to unstable $K$-theory $K^{*}$ for $A=Z\left[x, x^{-1}\right]$ and to the theory $H^{*}(, Z)$ for $A=Z$. The homology theories $U_{*}(, A)$ for $A=\Omega_{U}, Z[x], Z\left[x, x^{-1}\right], Z$, are isomorphic, respectively, to $U_{*}, k_{*}$, $K_{*}$ and $H_{*}(, Z)$.

Theorem 1. Since the complex $\left(S_{U}(Y), \partial\right)$ is a complex of free $\Omega_{U}$-modules, there exists a spectral sequence with term $E_{2}=\operatorname{Ext}_{\Omega_{U}}^{* *}\left(U_{*}(Y), A\right)$ which converges to $U^{*}(Y, A)$, and there exists a spectral sequence with term $E_{2}=\operatorname{Tor}_{\Omega_{U}}^{* *}\left(U_{*}(Y), A\right)$ which converges to $U_{*}(Y, A)$.

Theorem 2. Since the complexes $S_{U}(Y) \otimes_{\Omega_{U}} Z[x]=S_{k}(Y)$ and $S_{U}(Y) \otimes_{\Omega_{U}}$ $Z\left[x, x^{-1}\right]=S_{k}(Y)$ are complexes of free $A$-modules for $A=Z[x], Z\left[x, x^{-1}\right]$, and the ring $Z[x]$ is homologically onedimensional, we have the following universal coefficient formulas:

1) $0 \rightarrow \operatorname{Ext}_{Z[x]}^{2, *}\left(k_{*}, Z[x]\right) \rightarrow k^{*} \rightarrow \operatorname{Hom}_{Z[x]}^{*}\left(k_{*}, Z[x]\right) \rightarrow 0$

2) $0 \rightarrow k_{*} \otimes_{Z[x]} Z\left[x, x^{-1}\right] \rightarrow K_{*} \rightarrow \operatorname{Tor}_{Z[x]}^{1, *}\left(k_{*}, Z\left[x, x^{-1}\right]\right) \rightarrow 0$,

3) $0 \rightarrow k_{*} \otimes_{Z[x]} Z \rightarrow H_{*}(, Z) \rightarrow \operatorname{Tor}_{Z[x]}^{1, x}\left(k_{*}, Z\right)$,

where in formula 1) $k_{*}$ and $k^{*}$ are connected, in formula 2) $k_{*}$ and $K_{*}$, since $Z\left[x, x^{-1}\right]$ is a $Z[x]$-module, and in formula 3$) k_{*}$ and $H_{*}$, since $Z$ is a $Z[x]$-module. ${ }^{6}$

It is possible to find a number of other formulas connecting $k_{*}, k^{*}, K_{*}, K^{*}, H_{*}$, $H^{*}$ and also Künneth formulas for the direct product $Y_{1} \times Y_{2}$, starting with the complex $S_{U} \otimes_{\Omega} Z[x]$ as a $Z[x]$-module and the fact that $Z[x]$ is one-dimensional, as is $Z$.

We note also that $\operatorname{Hom}_{Z[x]}^{*}(Z[x], Z[x])=Z[y]$, where $\operatorname{dim} y=-2$.

In all the formulas of Corollary 2 one can start from the complex $\operatorname{Hom}_{\Omega_{U}}^{*}\left(S_{U}, Z[x]\right)$, which is a complex of free $Z[y]$-modules for $k^{*}$-theory.

With the help of the complex $S_{U}(Y)$ it is possible to introduce, in addition to the cohomological multiplication, also the "Čech operation" $\cap$ such that $(a \cap b, c)=$ $(a, b c)$, where $c, a \in U^{*}, b \in U_{*}$ and $a \cap b \in U_{*}$, while $(a \cap b, c) \in \Omega_{U}$. Analogously for $k_{*^{-}}$and $k^{*}-, K^{*}$ - and $K_{*}$-theories.

The Poincaré-Atiyah duality law, of course, is treated in the usual way by means of the fundamental cycle and the Čech operation.

\footnotetext{
${ }^{6}$ The author has available a derivation of Theorems 1 and 2 from the Adams spectral sequence in cobordism theory, and hence Theorems 1 and 2 do not depend on the preceding conjectures.
} 
We note that the homomorphisms $\sigma_{\omega}^{*}$ introduced by the author on the ring $\Omega_{U}$ represent "characteristic numbers" with values in $\Omega_{U}$, since the scalar product lies in $\Omega_{U}$.

\section{APPENDIX 4. $U_{*^{-}}$AND $k_{*}$-THEORY FOR $B G$, WHERE $G=Z_{m}$ FIXED POINTS OF TRANSFORMATIONS}

In this appendix we shall consider the following questions:

1. What are the cell complexes $S_{U}(B G)$ and $S_{k}(B G)$ for $G=Z_{m}$ ? What are the $\Lambda$-modules $U_{*}(B G)$ and $k_{*}(B G)$, where $\Lambda=\Omega_{U}$ and $\Lambda=Z[x]$ ?

2. How to compute in $U_{*}(B G)$ the following elements: let the group $Z_{m}$ act on $C^{n}$ linearly, and without fixed points on $C^{n} \backslash 0$, i.e., by means of diagonal matrices $\left(a_{i j}\right)$, where $a_{i j}=\exp \left(2 \pi i x_{j} / m\right)$ and $x_{j}$ is a unit in the ring $Z_{m}$. Then an action of $Z_{m}$ on $S^{2 n-1}$ is defined, and by the same token a map $f_{x_{1}, \ldots, x_{n}}: S^{2 n-1} / Z_{m} \rightarrow B G$, which represents an element of $U_{2 n-1}(B G)$. This element we denote by $\alpha_{n}\left(x_{1}, \ldots, x_{n}\right) \in U_{2 n-1}(B G)$. It is trivial to find $\alpha_{n}(1, \ldots, 1)$ ("geometric bordism") and to show that $\alpha_{n}\left(x_{1}, \ldots, x_{n}\right) \neq 0$ for all (invertible) $x_{1}, \ldots, x_{n} \in Z_{m}$ (see [11]),

$$
\nu \alpha_{n}\left(x_{1}, \ldots, x_{n}\right) \neq 0, \quad \nu: U_{*} \rightarrow H_{*}(, Z) .
$$

This question arises in connection with the Conner-Floyd approach to the study of fixed points (see [11]).

We consider the question of computing the cell complexes $S_{U}(B G), S_{k}(B G)$ and $S_{K}(B G)$ (see Appendix 3).

We recall the well-known result of Atiyah [7] that $K^{1}(B G)=0$ and $K^{0}(B G)=$ $R_{U} G^{\wedge}$, where $R_{U}(G)$ is the ring of unitary representations. For $G=Z_{m}$, the basic unitary representations $\rho_{0}=1, \rho_{1}=\left\{l^{2 \pi i / m}\right\}, \ldots, \rho_{k}=\left\{l^{2 \pi i k / m}\right\}, \ldots, \rho_{m-1}$ are one-dimensional, while as a ring a generator is $\rho_{1}=\rho$ with the relation $\rho^{m}=1$. By virtue of this we can choose in $K^{0}(B G)$ an element $t$, corresponding to $\rho-1$, with the relation $\Psi^{m}(t)=0$, where $\Psi_{*}^{m}$ is the Adams operator.

We consider the ring $k^{*}(P)=\operatorname{Hom}_{Z[x]}^{*}(Z[x], Z[x])=Z[y], \operatorname{dim} y=-2$. We have

Lemma 1. The $Z[y]$-module $k^{*}(B G)$ for $G=Z_{m}$ is described as follows:

a) $k^{2 j+1}=0$.

b) $k^{2 j}(B G)$ is isomorphic to the subgroup of $k^{0}(B G)$ consisting of elements of filtration $\geq 2 j$, an this isomorphism is established by the Bott operator $y^{j}$ :

$$
k^{2 j}(B G) \rightarrow K^{0}(B G) .
$$

c) The action of the rings $B_{\Psi}^{k}$ and $A_{\Psi}^{k}$ is well defined on $k^{*}(B G)$.

d) There exists a natural generator $u \in k^{2}(B G)$ such that every element of $k^{*}(B G)$ has the form $\sum_{j} y^{s_{j}} u^{q_{j}}$ and there is the relation $\left(m \Psi^{m}\right)(u)=0$, or $\Psi^{m}(y u)=0$, where $y u \in k^{0}=K^{0}$ is the canonical generator $t \in K^{0}(B G)$; and we have the equation

$$
\left(m \Psi^{m}\right)(u)=\sum_{k \geq 1}\left(\begin{array}{c}
m \\
k
\end{array}\right)(-y)^{k-1} u^{k} .
$$

The proof of the lemma follows easily from the results mentioned about $K^{0}(B G)$ and the discussion of the spectral sequence with term $E_{2}=H^{*}(B G, Z[y])$ which converges to $k^{*}(B G)$. 
We denote the expression $m \Psi^{m}(u)$ by $F_{m}(u)=\sum\left(\begin{array}{c}m \\ k\end{array}\right)(-y)^{k-1} u^{k}$. From Lemma 1 follows

Lemma 2. The cell complex $S_{k}^{*}(B G)=\operatorname{Hom}_{Z[x]}^{*}\left(S_{k}, Z[x]\right)$ of modules over $Z[y]$ is a ring with multiplicative generators (over $Z[y]$ ) $v$ (of dimension (1)) and $u$ (of dimension (2)) and additive basis $\left\{y^{s} u^{n}, y^{q} v u^{l}\right\}$. The differential $d$ in this complex satisfies the Leibnitz formula, commutes with multiplication by $y$ and has the form:

$$
d u=0, \quad d v=F_{m}(u) .
$$

The cell complex of $k$-theory $S_{k}(B G)$ for $G=Z_{m}$ in the natural cellular subdivision has the form $S_{k}(B G)=\operatorname{Hom}_{Z[y]}^{*}\left(S_{k}^{*}, Z[y]\right)$, while $S_{k}(B G)$ over $Z[x], Z[x]=$ $\operatorname{Hom}_{Z[y]}^{*}(Z[y], Z[x])$, is a complex of free modules.

Lemma 2 follows easily from Lemma 1 and Appendix.

We turn now to $U_{*^{-}}$and $U^{*}$-theories. For the element $u \in V(X)=$ $\operatorname{Map}\left(X, M U_{1}\right) \subset U^{2}(X)$, the series $m \Psi_{U}^{m}(u)=g^{-1}(m g(u))$ (see Appendix 2), where $g(u)=\sum_{n \geq 0}\left[C P^{n}\right] u^{n+1} /(n+1)$ is the "Miščenko series" (see Appendix 1).

We denote the series $m \Psi_{U}^{m}(u)$ by $F_{m, U}(u)$. Let

$$
\Lambda=U^{*}(P)=\operatorname{Hom}_{\Omega_{U}}^{*}\left(\Omega_{U}, \Omega_{U}\right),
$$

and let $S_{U}^{*}(B G)$ be the cell complex in $U^{*}$-theory which is a complex of $\Lambda$-modules, with $\Lambda^{-2 i}=\Omega_{U}^{2 i}$.

With the help of the Conner-Floyd homomorphism $\sigma_{1}: k^{0} \rightarrow U^{2}, k^{0}=K^{0}$, we obtain from Lemma 2

Theorem 1. The cell complex (in the natural cellular subdivision)

$$
S_{U}^{*}(B G)=\operatorname{Hom}_{\Omega_{U}}^{*}\left(S_{U}, \Omega_{U}\right),
$$

which is a complex of free $\Lambda$-modules, $\Lambda=U^{*}(P)$, with differential $d$, is a ring with multiplicative generators $v$ (of dimension (1)) and $u$ (of dimension (2)) over $\Lambda$, given in the following way:

$$
v^{2}=0, \quad d(v)=F_{m, U}(u), \quad d(u)=0 .
$$

The complex $S_{U}(B G)$ is isomorphic to $\operatorname{Hom}_{\Lambda}^{*}\left(S_{U}, \Lambda\right), G=Z_{m}$, where $\Omega_{U}=$ $\operatorname{Hom}_{\Lambda}^{*}(\Lambda, \Lambda)$. The complexes $S_{U}^{*} \otimes_{\Omega} Z[x]$ and $S^{*} \otimes_{\Omega} Z\left[x, x^{-1}\right]$ are isomorphic, respectively, to the complexes $S_{k}^{*}(B G)$ and $S_{k}^{*}(B G)$ in $k$ - and $K$-theories.

We pass now to the automorphisms of the complex $B G \rightarrow B G$. Such automorphisms for $G=Z_{m}$ are completely determined by automorphisms of the group $Z_{m} \rightarrow Z_{m}$, which are multiplication by $k$, where $k$ is a unit in $Z_{m}$.

There arise automorphisms

$$
\begin{aligned}
\lambda_{k}: B G & \rightarrow B G, \\
\lambda_{k}^{*}: S_{U}^{*}(B G) & \rightarrow S_{U}^{*}(B G),
\end{aligned}
$$

where $\lambda_{k}^{*}$ is completely determined by the images

$$
\lambda_{k}^{*}(v) \in S_{U}^{*}(B G), \quad \lambda_{k}^{*}(u) \in S_{U}^{*}(B G),
$$

since $\lambda_{k}^{*}$ is a ring homomorphism which commutes with the action of $\Lambda$.

We have 
Theorem 2. ${ }^{7}$ The homomorphism of multiplication by $k$

$$
\lambda_{k}^{*}: S_{U}^{*}(B G) \rightarrow S_{U}^{*}(B G)
$$

for $G=Z_{m}$ and $(k, m)=1$ is a ring homomorphism which commutes with $d$ and multiplication by $\Lambda$ and is defined by the following formulas:

a) $\lambda_{k}^{*}(u)=F_{k, U}(u)$,

b) $\lambda_{k}^{*}(v)=\frac{F_{k m, U}(u)}{F_{m, U}(u)} \cdot v$.

The proof of Theorem 2 is obtained from the fact that under $\lambda_{k}^{*}$ ("geometric cobordism") $u \in U^{2}(B G)$ must go into $k \Psi^{k}(u)$ by definition of the operator $\Psi^{k}$. This implies part (a). Part (b) follows from the fact that $d \lambda_{k}^{*}(v)=\lambda_{k}^{*} d v=D(u) v$, where $D(u)$ is a series of dimension 0 with coefficients in $\Lambda$.

We now pass to the question of fixed points of transformations $Z_{m}$. Let $Z_{m}^{*} \subset$ $Z_{m}$ be the multiplicative group of units, $x_{1}, \ldots, x_{n} \in Z_{m}^{*}$ and $\alpha_{n}\left(x_{1}, \ldots, x_{n}\right) \in$ $U_{2 n-1}(B G)$ the element defined by the linear action of the group $Z_{m}$ on $S^{2 n-1} \subset$ $C^{n} \backslash 0$ by means of multiplication of the $j$-th coordinate by $\exp \left(2 \pi i x_{j} / m\right), x_{j} \in Z_{m}^{*}$. There arises a function

$$
\alpha_{n}: Z_{m}^{*} \times \cdots \times Z_{m}^{*} \rightarrow U_{2 n-1}(B G) .
$$

Let $m=p^{n}, p$ a prime and $m_{1}=p^{n-1}$. Then $Z_{m_{1}} \subset Z_{m}$ and there is defined a homomorphism $U_{*}\left(B Z_{m_{1}}\right) \rightarrow U_{*}\left(B Z_{m}\right)$. We have

Lemma 3. Given a quasicomplex transformation $T: M^{n} \rightarrow M^{n}$ of order $m$ which has only isolated fixed points $P_{1}, \ldots, P_{q}$, we have the equation

$$
\sum_{j=1}^{q} \alpha_{n}\left(x_{1 j}, \ldots, x_{n j}\right) \equiv 0 \bmod U_{*}\left(B Z_{m_{1}}\right),
$$

where the $x_{i j}$ are the orders of the linear representation of the group $Z_{m}$ at the point $P_{j}$ (clearly, $\left.x_{i j} \in Z_{m}^{*}\right)$.

This lemma for prime $m=p$ was found by Conner-Floyd [11] (here, $m_{1}=0$ ), and it is trivial to go over to $m=p^{h}$.

It is easy to show that for any $\left(x_{1}, \ldots, x_{n}\right) \in Z_{m}^{*} \times \cdots \times Z_{m}^{*}$

$$
\alpha_{n}\left(x_{1}, \ldots, x_{n}\right) \not \equiv 0 \quad \bmod U_{*}\left(B Z_{m_{1}}\right),
$$

whence follows the theorem of Conner-Floyd-Atiyah: there does not exist a transformation $T$ with one fixed point. For $p>2$ this is also true for real transformations $T$, as can be seen from the analogous application of the theory $S O_{*} \otimes Z[1 / 2]$.

We now pass to the question of calculating the function $\alpha_{n}\left(x_{1}, \ldots, x_{n}\right) \in$ $U_{2 n-1}\left(B Z_{m}\right)$. We denote by $v_{2 n-1} \in U_{2 n-1}(B G)$ the so-called "geometric bordism" $\alpha_{n}(1, \ldots, 1)$. In the complex $S_{U}(B G)$ described in Theorem 1 , the element $v_{2 n-1}$ is adjoint to $v u^{n-1} \in S_{U}^{*}(B G)$, i.e., $\left(v_{2 n-1}, v u^{n-1}\right)=1,\left(v_{2 n-1}, v u^{n-1+k}\right)=0$ for $k>0$, where $x \in \Lambda^{*}$.

We shall calculate the function $\alpha_{n}\left(x_{1}, \ldots, x_{n}\right)$ by the following scheme:

1) Clearly, $\alpha_{1}(x)=x v_{1} \in U_{1}(B G)=Z_{m}$.

\footnotetext{
${ }^{7}$ All homological deductions from Theorems 1 and 2 of this appendix can be justified, without the complexes $S_{U}$, merely from Theorems 1 and 2 of Appendix 3.
} 
2) If $\sum_{j=1}^{m} \alpha_{k}\left(x_{1 j}, \ldots, x_{k j}\right) \equiv 0, \sum_{j=1}^{l} \alpha_{n-k}\left(y_{k+1, j}, \ldots, y_{n, j}\right) \equiv 0$, then we have the equation:

$$
\sum_{j, s} \alpha_{n}\left(x_{1 j}, \ldots, x_{k j}, y_{k+1, s}, \ldots, y_{n, s}\right) \equiv 0 \quad \bmod B Z_{m_{1}}
$$

This follows in an obvious way from the fact that transformations $T_{1}: M^{k} \rightarrow M^{k}$ and $T_{2}: M^{l} \rightarrow M^{l}$ induce $\left(T_{1}, T_{2}\right): M^{k} \times M^{n-k}$, where fixed points (and their orders) correspond to each other.

3) If $\lambda_{x}: B G \rightarrow B G$ is induced by multiplication by $x \in Z_{m}^{*}$, then $\alpha_{n}(x, \ldots, x)=$ $\lambda_{x, *}\left(v_{2 n-1}\right)$, where the structure of $\lambda_{x, *}$ is described in Theorem 2 .

As examples of the application of this scheme we shall indicate the following simple results:

Lemma 4. If $\nu: U_{*} \rightarrow H_{*}(, Z)$ is the natural homomorphism, then we have the equation

$$
\nu \alpha_{n}\left(x_{1}, \ldots, x_{n}\right)=\left(x_{1}, \ldots x_{n}\right) \nu\left(v_{2 n-1}\right),
$$

where $\nu\left(v_{2 n-1}\right) \in H_{2 n-1}\left(B Z_{m}\right)=Z_{m}$ is the basis element.

Lemma 5. For $n=1,2,3$ we have the formulas:

$$
\begin{gathered}
\lambda_{x, *}\left(v_{1}\right)=x v_{1}, \\
\lambda_{x, *}\left(v_{2}\right)=x^{2} v_{3}, \\
\lambda_{x, *}\left(v_{5}\right)=x^{3} v_{5}+\frac{x^{3}-x^{2}}{2}\left[C P^{1}\right] v_{3} .
\end{gathered}
$$

From Lemma 2, in an obvious way, follows the corollary on the impossibility of one fixed point.

Now let $m=p$, where $p>2$ for $n=2$ and $p>3$ for $n=3$. Under these conditions, by the scheme indicated above, one easily obtains from Lemmas 2 and 3

Theorem 3. The functions $\alpha_{n}\left(x_{1}, x_{2}, \ldots, x_{n}\right)$ for $n \leq 3$ has the following form:

$$
\begin{gathered}
\alpha_{1}(x)=x v_{1}(\text { obviously }) \\
\alpha_{2}\left(x_{1}, x_{2}\right)=\left(x_{1} x_{2}\right) v_{3} ; \\
\alpha_{3}\left(x_{1}, x_{2}, x_{3}\right)=\left(x_{1} x_{2} x_{3}\right) v_{5}+\frac{x_{1} x_{2} x_{3}-\frac{x_{1} x_{2}+x_{1} x_{3}+x_{2} x_{3}}{3}}{2}\left[C P^{1}\right] v_{3} .
\end{gathered}
$$

Suppose given a group of quasicomplex transformations $Z_{p}: M^{n} \rightarrow M^{n}$ with isolated fixed points $P_{1}, \ldots, P_{q}$ at which the generator $T \in Z_{p}$ has orders $x_{1 j}, \ldots, x_{n j} \in$ $Z_{p}, j=1, \ldots, q$, where $x_{k j} \in Z_{p}^{*}$. We consider the point $\left(x_{1,1}, \ldots, x_{k j}, \ldots, x_{n q}\right) \in$ $Z_{p}^{q n}$ up to a factor $\mu \in Z_{p}^{*}, \mu \neq 0$. Thus, $\left(x_{1,1}, \ldots, x_{n q}\right) \in P^{q n-1}$. The group $S_{n} \times S_{q}$, where $S_{k}$ is the group of permutations of $k$ elements, acts on $P^{q n-1}$.

Definition. By the type of the action of the group $Z_{p}$ on $M^{n}$ with isolated fixed points we shall mean the set of orders of $\left(x_{1,1}, \ldots, x_{k j}, \ldots, x_{n q}\right)$, of any generator $T \in Z_{p}$, considered in the projective space $P^{q n-1}$, factored by the actions of the group $S_{n}$ of permutations of orders of each point and the group $S_{q}$ of permutations of points.

From Theorem 3 follows the 
Corollary. For $p>n$ and for $n=2,3$, the set of types of actions of $Z_{p}$ on $M^{n}$ is given in $P^{q n-1}$ by the set of equations:

$$
\sum_{P_{j}} \sigma_{k}\left(x_{1 j}, \ldots, x_{n j}\right)=0, \quad x_{j s} \neq 0, \quad k=2,3, \ldots, n,
$$

where the $x_{s j}$ are the orders at the point $P_{j}$ and $\sigma_{k}\left(x_{1 j}, \ldots, x_{n j}\right)$ is the elementary symmetric polynomial.

\section{Appendix 5. The CONJECture on the BIGRAdATION OF ALgEBRAiC FUNCTORS \\ IN $S$-TOPOLOGY FOR ALL PRIMES $p>2$}

In the Introduction and also in $\S 12$ the possibility was already discussed of the appearance of a new categorical invariant - an additional grading, connected with the Cartan type, in the Adams spectral sequence for ordinary cohomology $\bmod p$, $p>2$, from which it would follow (see the Introduction) that the homotopy groups in the category of torsion-free complexes could be formally computed algebraically by the theory of unitary cobordism. We shall formulate here more exactly the corresponding conjecture.

First of all, we shall go to the question of the category $S \otimes_{Z} Q_{p}$ for $p>2$. Let $K(\pi) \in S$ be the spectrum $K(\pi, n)$. The following fact is known (H. Cartan): the Steenrod algebra $A=H^{*}\left(K\left(Z_{p}\right), Z_{p}\right)$ is bigraded: $A=\sum A^{k, \beta}$, where $\operatorname{dim}=k+\beta$ and $\beta$ is the type.

Conjecture: I) Let the bigradings $H\left(X, Z_{p}\right)=\sum H^{k, \beta}$ and $H\left(Y, Z_{p}\right)=\sum H^{k, \beta}$ be well defined, and let the morphism $f: X \rightarrow Y$ in the $S$-category preserve the bigrading. Then in the exact sequences

$$
0 \rightarrow X \stackrel{f}{\rightarrow} Y \rightarrow Z \rightarrow 0
$$

and

$$
0 \rightarrow Z^{\prime} \rightarrow X \stackrel{f}{\rightarrow} Y \rightarrow 0
$$

for the objects $Z, Z^{\prime} \in S$, the bigradings of the functors $H^{* *}\left(Z, Z_{p}\right)$ and $H^{* *}\left(Z^{\prime}, Z_{p}\right)$ are well defined, and the exact sequence of the triple $(X, Y, Z)$ is

$$
\cdots \rightarrow H^{k, \beta}(X) \stackrel{\delta}{\rightarrow} H^{k, \beta+1}(Z) \rightarrow H^{k, \beta+1}(Y) \stackrel{f^{*}}{\longrightarrow} H^{k, \beta+1}(X) \rightarrow \ldots
$$

II) For $X=K\left(Z_{p}\right)$ the bigrading coincides with that of Cartan.

III) The cohomology $A$-module $H^{*}\left(X, Z_{p}\right)$ is bigraded, if in $H^{*}\left(X, Z_{p}\right)$ the bigrading is well defined.

IV) All these properties are fulfilled in the subcategory $S_{g r} \subset S \otimes_{Z} Q_{p}$ obtained from $K\left(Z_{p}\right)$ inductively by means of bigraded morphisms and passage to "kernels" and "cokernels"

$$
0 \rightarrow X \stackrel{f}{\rightarrow} Y \rightarrow Z \rightarrow 0, \quad 0 \rightarrow Z^{\prime} \rightarrow X \stackrel{f}{\rightarrow} Y \rightarrow 0 ;
$$

here $Q_{p}$ is the $p$-adic integers.

Assertion. 1. If the conjecture is true, then the spectra of points (spheres) $P$ and complexes without $p$-torsion in homology belong to the category $S_{g r}$.

2. If the analogous conjecture of bigradation for other functors (for example, homotopy groups) is true, then the entire classical Adams spectral sequence and the stable homotopy groups of spheres for $p>2$ can be completely calculated by means 
of the theory of unitary cobordism by the scheme described in the Introduction and in $\S 12$. In particular, we should have the equation:

$$
\pi_{* *}^{(p)}\left(S^{N}\right) \approx \operatorname{Ext}_{A^{U}}^{* *}(\Lambda, \Lambda) \otimes_{Z} Q_{p}, \quad p>2 .
$$

This means two things: a) the triviality of the Adams spectral sequence constructed by the author in the theory of $U$-cobordism; b) the absence of extensions in the term $E_{\infty}=E_{2}$.

3. For $p=2$, the conjecture in such a simple form is trivially false. In the spectral sequence for the stable groups of spheres, all powers $\eta^{k} \neq 0$ for an element $\eta$ representing the Hopf map in $\pi_{1}(S)$, hence $\eta^{k}$ for $k \geq 4$ must be killed off by differentials.\}

4. The classical Adams spectral sequence with second term $E_{2}^{s, k, \beta}=$ $\operatorname{Ext}_{A}^{s, k, \beta}\left(H^{* *}(X), Z_{p}\right)$ for $X \in S_{g r}$ is arranged as follows:

$$
d_{r}: E_{r}^{s, k, \beta} \rightarrow E_{r}^{s+r, k, \beta+r-1}
$$

We note that $h_{0} \in \operatorname{Ext}_{A}^{1,0,1}\left(Z_{p}, Z_{p}\right)$ is associated with multiplication by $p$ (ordinarily we have $h_{0} \in \operatorname{Ext}_{A}^{1,1}$ ). Here, the dimension differs slightly from that described in $\S 12$ by a simple linear substitution.

Examples of bigradation (the simplest). Let $X=K(Z)+E K(Z)$ and $Y=K\left(Z_{p^{q}}\right)$. From the ordinary point of view we have:

$$
H^{*}\left(X, Z_{p}\right)=H^{*}\left(Y, Z_{p}\right)=A / A \beta(u)+A / A \beta(v)
$$

where $\operatorname{dim} u=0$ and $\operatorname{dim} v=1$. However, for $X$ the ordinary Adams spectral sequence is zero, but for $Y$ we have: $d_{q}\left(v^{*}\right)=h_{0}^{q} u^{*}$, where $u^{*} \in \operatorname{Ext}_{A}^{0,0}$ and $v^{*} \in$ $\operatorname{Ext}_{A}^{0,1}$, since $\pi_{*}(Y)=Z_{p^{q}}$.

From our point of view the situation is thus:

a) $H^{* *}\left(X, Z_{p}\right)=A / A \beta(u)+A / A \beta(v)$, where $u \in H^{0,0}, v \in H^{0,1}$. Hence $u^{*} \in$ $\operatorname{Ext}_{A}^{0,0,0}, v^{*} \in \operatorname{Ext}_{A}^{0,1,0}$ and $h_{0}^{q} u^{*} \in \operatorname{Ext}^{q, q, 0} ;$ by dimensional considerations, $d_{q}\left(v^{*}\right) \in$ $\operatorname{Ext}_{A}^{q, 1, q-1}$, and $\operatorname{Ext}_{a}^{q, 1, q-1}=0$.

b) $H^{* *}\left(Y, Z_{p}\right)=A / A \beta(u)+A / A \beta(v), u \in H^{0,0}, v \in H^{0,1}$, then $u^{*} \in \operatorname{Ext}_{A}^{0,0,0}$ and $v^{*} \in \operatorname{Ext}_{A}^{0,0,1}, d_{i} v^{*} \neq 0$ for $i=q$.

Besides the facts indicated earlier, there are subtler circumstances which corroborate the conjecture:

1. From the results of the author's series of papers on the $J$-homomorphism $J_{*} \subset \pi_{*}\left(S^{N}\right)$ and the results of the present paper, it follows that $\operatorname{Ext}_{A^{U}}^{1, *}(\Lambda, \Lambda) \otimes_{Z} Q_{p}$ consists (for $p>2$ ) of cycles for all differentials, while elements of $\operatorname{Ext}_{A^{U}}^{1, *}$ are realized by elements of $\pi_{*}^{(p)}\left(S^{N}\right)$ of the same order; moreover, $\pi_{*}^{(p)}\left(S^{N}\right)=\operatorname{Ext}_{A^{U}}^{1, *}+\ldots$, where $\operatorname{Ext}^{1, *}=J_{*} \otimes_{Z} Q_{p}$.

2. The Adams spectral sequence in $U$-theory would not have to be trivial from dimensional considerations (obviously, only $d_{i}$ is zero for $i-1 \equiv 0 \bmod 2 p-2$ ). There first appears an element $x \in \operatorname{Ext}_{A^{U}}^{2,2 p^{2}(p-1)}$ where $d_{2 p-1}(x)=$ ?, since $\operatorname{Ext}_{A^{U}}^{2 p+1,2 p^{2}(p-1)+2 p-2} \neq 0$. In reality, these elements in $U$-theory are "inherited" from ordinary cohomology theory $H^{*}\left(, Z_{p}\right)$ together with the question about $d_{2 p-1}(x)$. A few years ago L. N. Ivanovskil informed the author that with the help of partial operations of Adams type he had succeeded in showing that $d_{2 p-1}(x)=0$ for $p>3$ (?). However, neither Ivanovskiu nor the author were able to verify this calculation, and hence this fact remained obscure. Recently Peterson informed the 
author that it has only recently been proved by the young American topologist Cohen [25] for all $p \geq 3$ (more exactly, the analogue of this question in the classical theory, from which, of course, it follows).

3. The fact that the "algebraic" Adams spectral sequence associated with the "topological" one, which begins with $E_{2}=\operatorname{Ext}_{A}^{* * *}\left(Z_{p}, Z_{p}\right)$ and converges to $\operatorname{Ext}_{A^{U}}^{* *}(\Lambda, \Lambda) \otimes_{Z} Q_{p}$ (see $\S 12$ ), is algebraically well-defined, is non-trivial a priori. The situation here is that if for some spectral sequence $\left(E_{r}, d_{r}\right)$ we consider the complementary filtration in $E_{2}$ and define on all the $\bar{E}_{r}$ associated differentials $\bar{d}_{r}$, then very often the $\bar{d}_{r}$ are not included in a well-defined spectral sequence (of algebras). Hence the fact of such a well-defined inclusion is in our case an extra geometric argument for the existence of an invariant second grading in the subcategory $S_{g r} \subset S \otimes_{Z} Q_{p}$.

\section{REFERENCES}

[1] J. F. Adams, On the structure and applications of the Steenrod algebra, Comment. Math. Helv. 32 (1958), 180-214. MR 20 \#2711.

[2] _ - Vector fields on spheres, Ann. of Math. (2) 75 (1962), 603-632. MR 25 \#2614.

[3] $\quad$ On the groups J(I). I, Topology 2 (1963), 181-195. MR 28 \#2553.

[4] J. F. Adams and M. F. Atiyah, K-theory and the Hopf invariant, Quart. J. Math. Oxford Ser. (2) 17 (1966), 31-38. MR 33 \#6618.

[5] M. F. Atiyah, Bordism and cobordism, Proc. Cambridge Philos. Soc. 57 (1961), 200-208. MR 23 \#A4150.

[6] _ Thom complexes, Proc. London Math. Soc. (3) 11 (1961), 291-310. MR 24 \#A1727.

[7] _ Characters and cohomology of finite groups, Inst. Hautes Études Sci. Publ. Math. No. 9 (1961), 23-64. MR 26 \#6228.

[8] M. F. Atiyah and F. Hirzebruch, Riemann-Roch theorems for differentiable manifolds, Bull. Amer. Math. Soc. 65 (1959), 276-281. MR 22 \#989.

[9] D. W. Anderson, E. Brown and F. Peterson, SU-cobordism, KO-characteristic numbers and the Kervaire invariant, Ann. of Math. (2) 83 (1966), 54-67. MR 32 \#6470.

[10] _ Spin cobordism, Bull. Amer. Math. Soc. 72 (1966), 256-260. MR 32 \#8349.

[11] P. Conner and E. Floyd, Cobordism theories, Princeton Univ. Press, Princeton, N. J., 1963, pp. 1-15. Preprint.

[12] _ The SU-bordism theory, Bull. Amer. Math. Soc. 70 (1964), 670-675. MR 29 \#5253.

[13] _ Torsion in SU-bordism, Mem. Amer. Math. Soc. No. 60 (1966), 1-73. MR 32 \#6471.

[14] M. Kervaire and J. Milnor, Bernoulli numbers, homotopy groups and a theorem of Rohlin, Proc. Internat. Congr. Math., 1958, Cambridge Univ. Press, New York, (1960), pp. 454-458. MR 22 \#5537.

[15] J. Milnor, On the cobordism ring $\Omega^{*}$ and a complex analogue. I, Amer. J. Math. 82 (1960), 505-521. MR 22 \#9975.

[16] A. S. Miščenko, Spectral sequences of Adams type, Mat. Zametki 1 (1967), 339-346. (Russian) MR 35 \#2286.

[17] S. P. Novikov, Some problems in the topology of manifolds connected with the theory of Thorn spaces, Dokl. Akad. Nauk SSSR 132 (1960), 1031-1034 = Soviet Math. Dokl. 1 (1960), 717-720. MR 22 \#12545.

[18] _ Homotopy properties of Thom complexes, Mat. Sb. 57 (99) (1962), 407-442. (Russian) MR 28 \#615.

[19] , Rings of operations and spectral sequences of Adams type in extraordinary cohomology theories. $U$-cobordism and $K$-theory, Dokl. Akad. Nauk SSSR 172 (1967), 33-36= Soviet Math. Dokl. 8 (1967), 27-31. MR 36 \#884.

[20] V. A. Rohlin, Intrinsic homology, Dokl. Akad. Nauk SSSR 89 (1953), 789-792. (Russian). MR 15, 53

[21] _ Intrinsic homology theory, Uspehi Mat. Nauk 14 (1959), no. 4 (88), 3-20; English transl. Amer. Math. Soc. Transl. (2) 30 (1963), 255-271. MR 22 \#11402; MR 27 \#5252.

[22] R. Stong, Relations among characteristic numbers. I, Topology 4 (1965), 267-281. MR 33 \#740. 
[23] C. T. C. Wall, Determination of the cobordism ring, Ann. of Math. 72 (I960), 292-311. MR 22 \#11403.

[24] S. P. Novikov, Cohomology of the Steenrod algebra, Dokl. Akad. Nauk SSSR 128 (1959), 893-895 (Russian). MR 22 \#1889.

[25] J. Cohen, Some results on the stable homotopy groups of spheres, Bull. Amer. Math. Soc. 72 (1966), 732-735. MR 35 \#1022.

[26] E. Brown and F. Peterson, A spectrum whose $Z_{p}$ cohomology is the algebra of reduced $p^{\text {th }}$ powers, Topology 5 (1966), 149-154. MR 33 \#719. 